\title{
UV-vis spectroscopy as a tool for the detection of residual polymer and optimization of polymer dose in drinking water treatment applications
}

\author{
By \\ Renay Cormier
}

A thesis submitted to the Faculty of Graduate and Postdoctoral Affairs in partial fulfillment of the requirements for the degree of

Master of Applied Science

In

Environmental Engineering

Carleton University

Ottawa, ON

(C)2019

Renay Cormier 


\begin{abstract}
The optimization of polymer dose during the coagulation and flocculation stages of drinking water treatment remains a challenge for treatment plants. Incorrect polymer dosage can lead to either inadequate flocculation or the restabilization of flocs, filter or membrane clogging downstream, potential health effects due to the toxicity of polymers, and increased polymer costs. Analytical methods are not suitable for on-site monitoring and optimization of polymer dose, and it is particularly challenging to accurately detect and measure very low polymer concentrations in drinking water. This study employed an in-line and real-time UV-vis spectrophotometer to measure the polymer concentration in raw and treated water by correlating polymer concentration to the absorbance at $190 \mathrm{~nm}$ and introduced a new approach to improve the sensitivity and detection limit of the method through altering the path length. In addition, the method was used to determine the optimum polymer dose required for treatment to prevent the addition of excess polymer during coagulation/flocculation. The method has the potential for development into a full-scale optimization system.
\end{abstract}




\section{LIST OF ABBREVIATIONS}

A

AU

$\mathrm{C}$

CD

CST

$\mathrm{ECH}$

EPA

GC

H-Bond

HPLC

I

$I_{o}$

$L$

MS

MW

NOM

NTU

PAM

PDADMAC

PDA

PL sensor

RI

RSD

SCD

SEC

$T$

UV-vis

$\varepsilon$

$\xi$

$\theta$

Absorbance

Absorbance units

Concentration

Charge density

Capillary suction time

Epichlorohydrin

Environmental Protection Agency (United States)

Gas chromatography

Hydrogen bond

High-performance liquid chromatography

Intensity

Incident intensity

Path length

Mass spectrometry

Molecular weight

Natural organic matter

Nephelometric Turbidity Units

Polyacrylamide

Polydiallyldimethylammonium chloride

Photometric dispersion analyzer

Power line sensor

Refractive index

Relative standard deviation

Streaming current detection

Size exclusion chromatography

Transmittance

Ultraviolet-visible

Molar absorptivity

Zeta potential

Fractional surface coverage 


\section{AKNOWLEDGEMENTS}

I would foremost like to express my gratitude to my Supervisor, Dr. Banu Örmeci, for her incredible academic support and guidance throughout the duration of this study. Secondly, I would like to thank my parents for their undying support in all of my endeavors, academic or otherwise. I would also like to thank Carleton's Graduate Environmental Engineering Laboratory Supervisor, Marie Tudoret, for her kind assistance during the conduction of the experimental research. I would like to thank Research Associate, Richard Kibbie, for his guidance and advice during the experimental phases of this study. Furthermore, I would like to extend my thanks to my friend and colleague, Ashley Piché, for her companionship and advice in the completion of our graduate and undergraduate degrees. Finally, I would like to thank my friend and partner, Nick Smith, for his support and encouragement in this study and all my ventures. Without the above-mentioned individuals, this study would not have been possible. 


\section{TABLE OF CONTENTS}

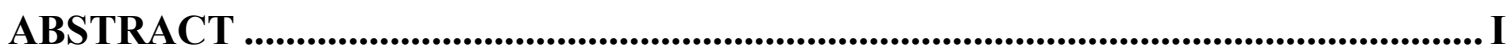

LIST OF ABBREVIATIONS............................................................................................II

AKNOWLEDGEMENTS .............................................................................................. III

LIST OF TABLES................................................................................................................ VI

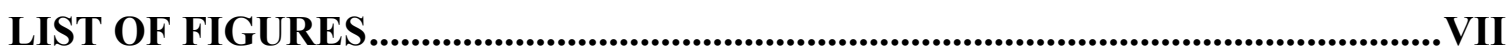

CHAPTER 1: INTRODUCTION .................................................................................

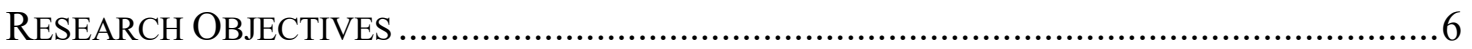

Phase 1: Effect of path length on polymer detection using UV-vis spectroscopy ....... 7

Phase 2: Effect of filtration and dilution on polymer detection using UV-vis

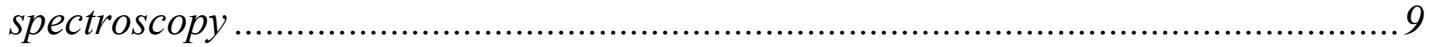

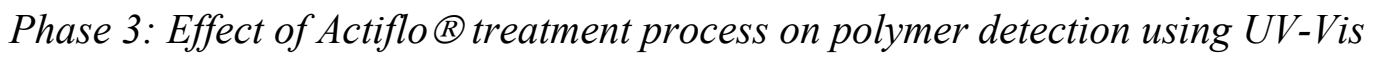

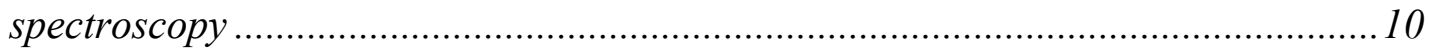

Phase 4: Determination of optimum polymer dose using $U V$-vis spectroscopy .......11

1.2 THESIS LAYOUT ............................................................................................12

CHAPTER 2: LITERATURE REVIEW ...................................................................14

2.1 APPLICATIONS OF POLYMERS IN DRINKING WATER TREATMENT .........................14

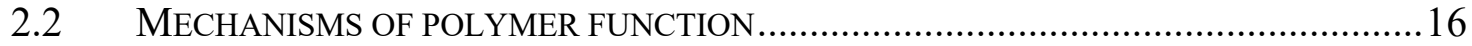

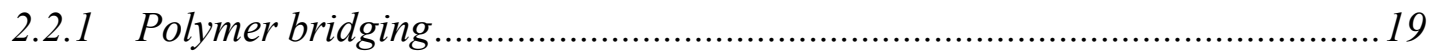

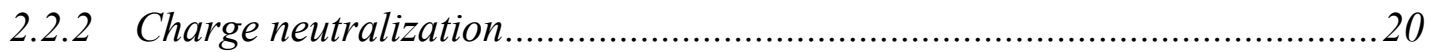

2.3 OPERATIONAL AND ENVIRONMENTAL DISADVANTAGES OF POLYMER IN DRINKING

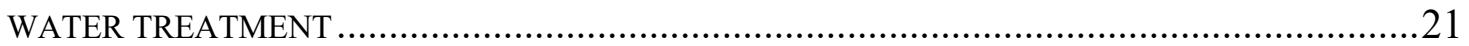

2.4 CURRENT Polymer Dose Monitoring Systems IN Drinking WATER

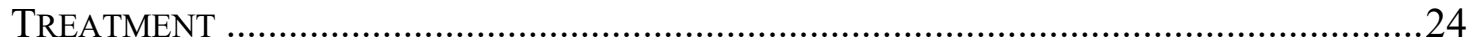

2.5 UV-VIS SPECTROSCOPY AS A POLYMER DOSE MONITORING METHOD ..................27

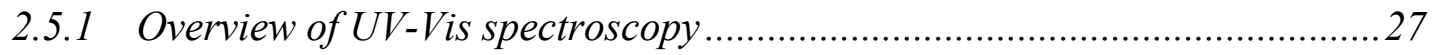

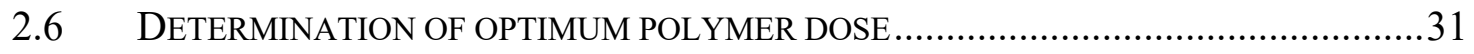

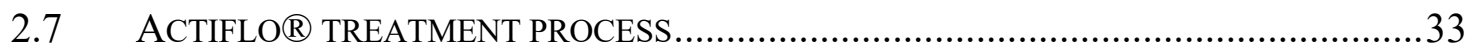

CHAPTER 3: MATERIALS AND METHODS ..............................................................35

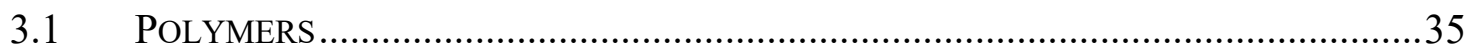

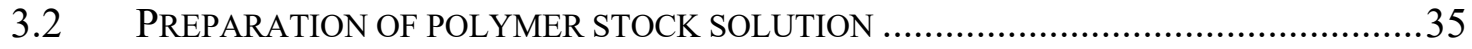

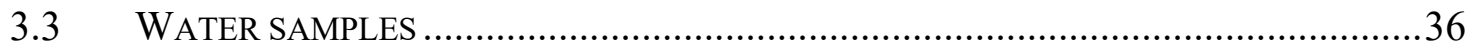

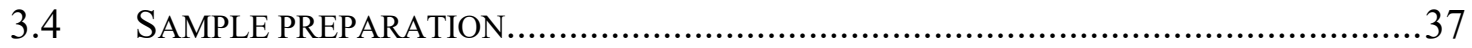

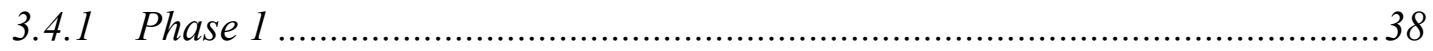

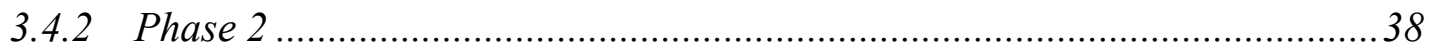




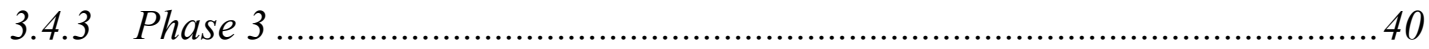

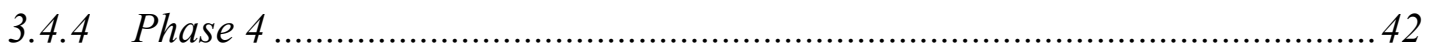

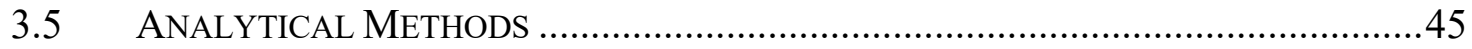

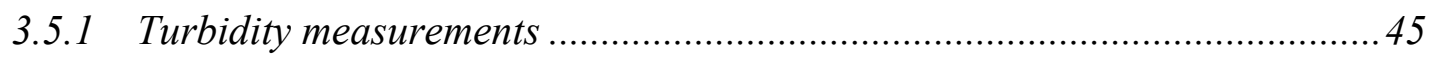

3.5.2 UV-Vis Spectroscopy Absorbance measurements .........................................

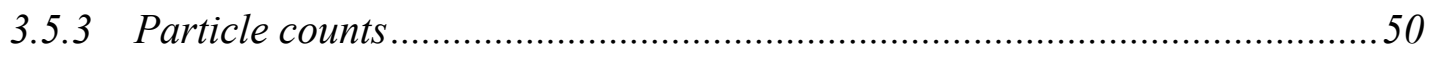

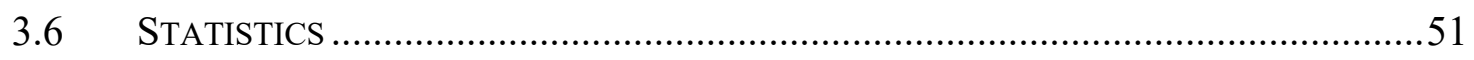

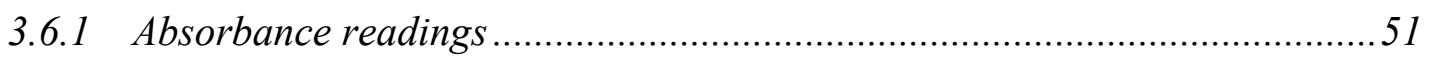

CHAPTER 4: RESULTS \& DISCUSION .................................................................52

4.1 EFFECT OF PATH LENGTH ON POLYMER DETECTION USING UV-VIS SPECTROSCOPY 52

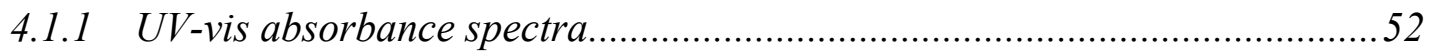

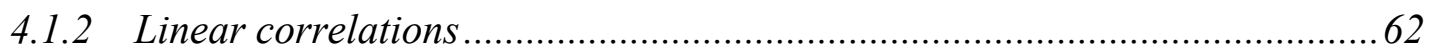

4.2 EFFECT OF PARTICLES ON POLYMER DETECTION USING UV-VIS SPECTROSCOPY 69

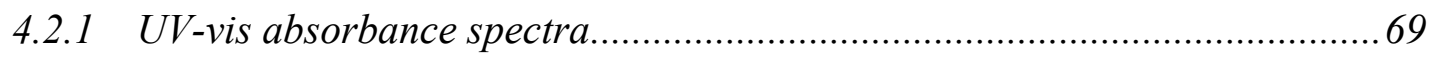

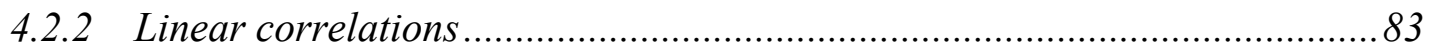

4.3 EFFECT OF WATER TREATMENT PROCESS CHEMICALS ON POLYMER DETECTION

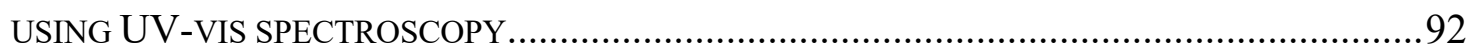

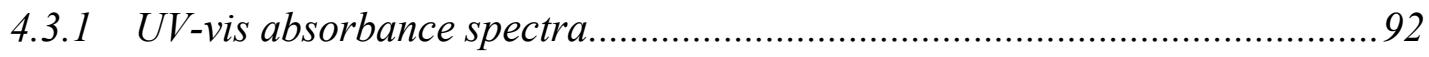

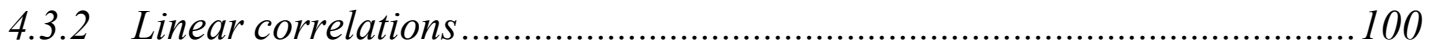

4.4 DETERMINATION OF OPTIMUM POLYMER DOSE IN DRINKING WATER TREATMENT

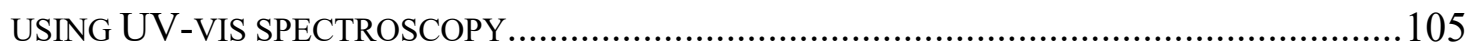

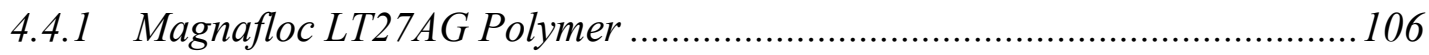

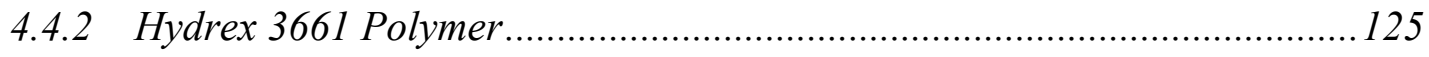

4.4.2 UV-vis absorbance as an in-line method for polymer optimization.............140

4.5 DISCUSSION OF UV-VIS SPECTROSCOPY IN COMPARISON TO OTHER ANALYTICAL METHODS FOR THE DETECTION OF RESIDUAL POLYMER IN DRINKING WATER TREATMENT

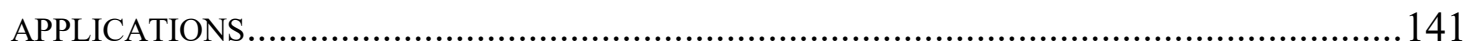

4.6 DISCUSSION OF DETERMINATION OF OPTIMUM POLYMER DOSE …........................144

CHAPTER 6: CONCLUSION .............................................................................146

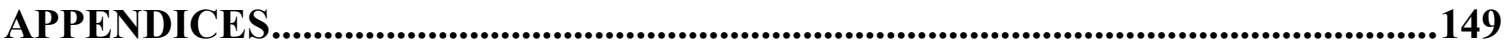

APPENDIX A: UV-VIS ABSORBANCE DATA FOR EXPERIMENTAL PHASE 1 ......................149

APPENDIX B: UV-VIS ABSORBANCE DATA FOR EXPERIMENTAL PHASE 2 ......................159

APPENDIX C: UV-VIS ABSORBANCE DATA FOR EXPERIMENTAL PHASE 3 ......................179

APPENDIX D: UV-VIS ABSORBANCE DATA FOR EXPERIMENTAL PHASE 4 …....................191

APPENDIX E: PARTICLE COUNT DATA FOR EXPERIMENTAL PHASE $4 \ldots \ldots \ldots \ldots \ldots \ldots \ldots \ldots \ldots \ldots . . . . .206$

REFERENCES ...............................................................................................................208 


\section{LIST OF TABLES}

Table 1: Current methods for polymer monitoring in drinking water treatment applications ...................25

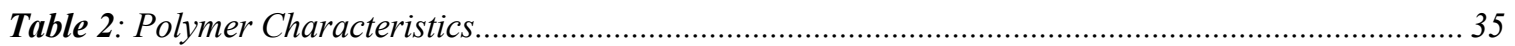

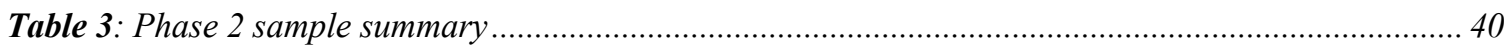

Table 4: Actiflo treatment process additives summary .................................................................... 41

Table 5: Summary of absorbance spectra data results at 190nm for path length comparison using the desktop and in-line spectrophotometers at the high concentration range of Magnafloc LT27AG polymer (0-

$3 \mathrm{mg} / \mathrm{L})$.

Table 6: Summary of absorbance spectra data results at 190nm for path length comparison using the desktop and in-line spectrophotometers at the low concentration range of Magnafloc LT27AG polymer (0-1 $m g / L)$......

Table 7: Summary of coefficient of determinations for desktop and in-line UV-vis spectrophotometers at a high Magnafloc LT27AG polymer concentration range (0-3 $\mathrm{mg} / \mathrm{L})$

Table 8: Summary of coefficient of determinations for desktop and in-line UV-vis spectrophotometers at a low Magnafloc LT27AG polymer concentration range $(0-1 \mathrm{mg} / \mathrm{L})$......

Table 9: Summary of absorbance spectra data results for effect of particles on UV-Vis absorbance for the detection of residual polymer using the desktop and in-line spectrophotometers at the high concentration range of Magnafloc LT27AG polymer $(0-3 \mathrm{mg} / \mathrm{L})$.....

Table 10: Summary of absorbance spectra data results for effect of particles on UV-Vis absorbance for the detection of residual polymer using the desktop and in-line spectrophotometers at the low concentration range of Magnafloc LT27AG polymer $(0-1 \mathrm{mg} / \mathrm{L})$.....

Table 11: Summary of coefficient of determinations for desktop and in-line UV-vis spectrophotometers at a high Magnafloc LT27AG polymer concentration range (0-3 mg/L) in Rideau River water samples .......... 91 Table 12: Summary of coefficient of determinations for desktop and in-line UV-vis spectrophotometers at a low Magnafloc LT27AG polymer concentration range (0-1 mg/L) in Rideau River water samples.

Table 13: Settling test data for high turbidity (9172 NTU) Rideau River Water dosed with Magnafloc LT27AG polymer.

Table 14: Settling test data for low turbidity (260 NTU) Rideau River Water dosed with Magnafloc LT27AG polymer.

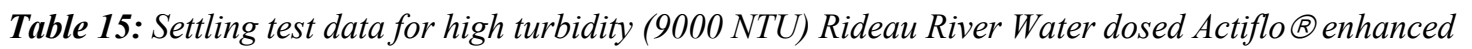
coagulation process chemicals and Magnafloc LT27AG polymer.

Table 16: Settling test data for high turbidity (4426 NTU) Rideau River Water dosed with Hydrex 3661 polymer.

Table 17: Settling test data for low turbidity (546 NTU) Rideau River Water dosed with Hydrex 3661 polymer

Table 18: Comparison of optimum polymer dosages determined using the four methods in all water samples. 


\section{LIST OF FIGURES}

Figure 1: UV-vis spectroscopy as a tool for the in-line and real time monitoring of residual polymer in drinking water treatment applications schematic......

Figure 2: UV-vis spectroscopy as a tool for the in-line and real time optimization of residual polymer in drinking water treatment applications schematic.....

Figure 3: General drinking water treatment processes ..................................................................... 14

Figure 4: A negative colloidal particle with its electrostatic field demonstrating the electrical double layer (Forbes, E., Chryss, A., Clays in the Minerals Processing Value Chain, 2017)...................................... 17

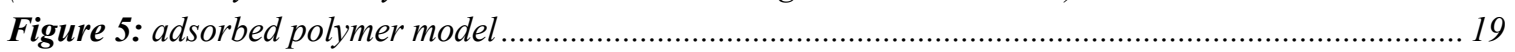

Figure 6: Polymer bringing mechanism schematic (Singh, H. et al., High-technology materials based on modified polysaccharides, 2009) .................................................................................................. 19

Figure 7: Electromagnetic spectrum schematic (National Geographic, 2018).......................................28

Figure 8: UV-Vis spectrophotometer internal workings schematic (Sigma Aldrich, USA)......................29

Figure 9: Actiflo clarification process and Dunsenflo filtration unit schematic (Casselman Treatment

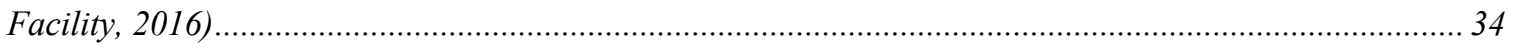

Figure 10: Experimental phase outline schematic ......................................................................... 37

Figure 11: In-line spectrophotometer (Real Tech Inc.) ....................................................................... 46

Figure 12: Absorbance spectra of Magnafloc LT27AG polymer in deionized water using a desktop spectrophotometer with a $10 \mathrm{~mm}$ flow cell in the concentration ranges of (a) 0-3 mg/L and (b) 0-1 $\mathrm{mg} / \mathrm{L} \mathrm{..} \mathrm{53}$

Figure 13: Absorbance spectra of Magnafloc LT27AG polymer in deionized water using an in-line spectrophotometer with a $2 \mathrm{~mm}$ flow cell in the concentration ranges of (a) $0-3 \mathrm{mg} / \mathrm{L}$ and (b) $0-1 \mathrm{mg} / \mathrm{L} \ldots .54$ Figure 14: Absorbance spectra of Magnafloc LT27AG polymer in deionized water using an in-line spectrophotometer with a $4 \mathrm{~mm}$ flow cell in the concentration ranges of (a) $0-3 \mathrm{mg} / \mathrm{L}$ and (b) $0-1 \mathrm{mg} / \mathrm{L} \mathrm{\ldots ..} 54$ Figure 15: Absorbance spectra of Magnafloc LT27AG polymer in deionized water using an in-line spectrophotometer with an $8 \mathrm{~mm}$ flow cell in the concentration ranges of (a) $0-3 \mathrm{mg} / \mathrm{L}$ and (b) $0-1 \mathrm{mg} / \mathrm{L}$.. 55 Figure 16: Linear correlation of the absorbance-polymer concentration data of Magnafloc LT27AG in deionized water at 190nm using a desktop spectrophotometer with a 10mm flow cell in the concentration ranges of (a) 0-3 $\mathrm{mg} / \mathrm{L}$ and (b) $0-1 \mathrm{mg} / \mathrm{L}$

Figure 17: Linear correlation of the absorbance-polymer concentration data of Magnafloc LT27AG in deionized water at 190nm using an in-line spectrophotometer with a $2 \mathrm{~mm}$ flow cell in the concentration ranges of (a) 0-3 $\mathrm{mg} / \mathrm{L}$ and (b) $0-1 \mathrm{mg} / \mathrm{L}$

Figure 18: Linear correlation of the absorbance-polymer concentration data of Magnafloc LT27AG in deionized water at 190nm using an in-line spectrophotometer with a $4 \mathrm{~mm}$ flow cell in the concentration ranges of (a) $0-3 \mathrm{mg} / \mathrm{L}$ and (b) $0-1 \mathrm{mg} / \mathrm{L}$

Figure 19: Linear correlation of the absorbance-polymer concentration data of Magnafloc LT27AG in deionized water at 190nm using an in-line spectrophotometer with an $8 \mathrm{~mm}$ flow cell in the concentration ranges of (a) $0-3 \mathrm{mg} / \mathrm{L}$ and (b) $0-1 \mathrm{mg} / \mathrm{L}$.....

Figure 20: Absorbance spectra of Magnafloc LT27AG polymer in unfiltered and undiluted Rideau River water with a turbidity of 6.8NTU using a desktop spectrophotometer with a $10 \mathrm{~mm}$ flow cell in the concentration ranges of (a) $0-3 \mathrm{mg} / \mathrm{L}$ and (b) $0-1 \mathrm{mg} / \mathrm{L}$.

Figure 21: Absorbance spectra of Magnafloc LT27AG polymer in unfiltered and undiluted Rideau River water with a turbidity of 6.8NTU using an in-line spectrophotometer with a $4 \mathrm{~mm}$ flow cell in the concentration ranges of (a) $0-3 \mathrm{mg} / \mathrm{L}$ and (b) $0-1 \mathrm{mg} / \mathrm{L}$.

Figure 22: Absorbance spectra of Magnafloc LT27AG polymer in filtered (0.45 $\mathrm{mm}$ ) and undiluted Rideau River water with an initial turbidity of 6.8NTU using a desktop spectrophotometer with a $10 \mathrm{~mm}$ flow cell in the concentration ranges of (a) $0-3 \mathrm{mg} / \mathrm{L}$ and (b) $0-1 \mathrm{mg} / \mathrm{L}$.... 
Figure 23: Absorbance spectra of Magnafloc LT27AG polymer in filtered $(0.45 \mu \mathrm{m})$ and undiluted Rideau River water with an initial turbidity of 6.8NTU using an in-line spectrophotometer with a $4 \mathrm{~mm}$ flow cell in the concentration ranges of (a) $0-3 \mathrm{mg} / \mathrm{L}$ and (b) $0-1 \mathrm{mg} / \mathrm{L}$

Figure 24: Absorbance spectra of Magnafloc LT27AG polymer in unfiltered Rideau River water diluted 1:1 with deionized water with an initial turbidity of 3.2NTU using a desktop spectrophotometer with a $10 \mathrm{~mm}$ flow cell in the concentration range of $0-1 \mathrm{mg} / \mathrm{L}$.

Figure 25: Absorbance spectra of Magnafloc LT27AG polymer in unfiltered Rideau River water diluted 1:1 with deionized water with an initial turbidity of $3.2 N T U$ using an in-line spectrophotometer with a $4 \mathrm{~mm}$ flow cell in the concentration ranges of (a) $0-3 \mathrm{mg} / \mathrm{L}$ and (b) $0-1 \mathrm{mg} / \mathrm{L}$.

Figure 26: Absorbance spectra of Magnafloc LT27AG polymer in filtered Rideau River water filtered with a $0.45 \mu \mathrm{m}$ filter and diluted 1:1 with deionized water with an initial turbidity of 3.2NTU using a desktop

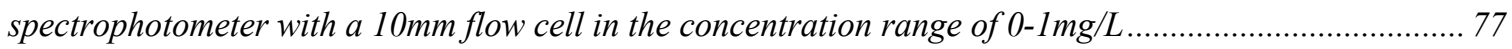
Figure 27: Absorbance spectra of Magnafloc LT27AG polymer in filtered Rideau River water filtered with a $0.45 \square m$ filter and diluted 1:1 with deionized water with an initial turbidity of $3.2 N T U$ using an in-line spectrophotometer with a $4 \mathrm{~mm}$ flow cell in the concentration ranges of (a) $0-3 \mathrm{mg} / \mathrm{L}$ and (b) $0-1 \mathrm{mg} / \mathrm{L} \ldots . . .78$ Figure 28: Linear correlation of the absorbance-polymer concentration data of Magnafloc LT27AG in Rideau River water with a turbidity of 6.8NTU at 190nm using a desktop spectrophotometer with a 10mm flow cell in the concentration ranges of (a) $0-3 \mathrm{mg} / \mathrm{L}$ and (b) $0-1 \mathrm{mg} / \mathrm{L}$.......

Figure 29: Linear correlation of the absorbance-polymer concentration data of Magnafloc LT27AG in Rideau River water with a turbidity of $6.8 N T U$ at 190nm using an in-line spectrophotometer with a $4 \mathrm{~mm}$ flow cell in the concentration ranges of (a) $0-3 \mathrm{mg} / \mathrm{L}$ and (b) $0-1 \mathrm{mg} / \mathrm{L}$.....

Figure 30: Linear correlation of Magnafloc LT27AG polymer in filtered $(0.45 \mu \mathrm{m})$ and undiluted Rideau River water with an initial turbidity of $6.8 N T U$ using a desktop spectrophotometer with a $10 \mathrm{~mm}$ flow cell in the concentration ranges of (a) $0-3 \mathrm{mg} / \mathrm{L}$ and (b) $0-1 \mathrm{mg} / \mathrm{L}$.

Figure 31: Linear correlation of Magnafloc LT27AG polymer in filtered $(0.45 \mu \mathrm{m})$ and undiluted Rideau River water with an initial turbidity of 6.8NTU using an in-line spectrophotometer with a $4 \mathrm{~mm}$ flow cell in the concentration ranges of (a) $0-3 \mathrm{mg} / \mathrm{L}$ and (b) $0-1 \mathrm{mg} / \mathrm{L}$

Figure 32: Linear correlation of Magnafloc LT27AG polymer in unfiltered Rideau River water diluted 1:1 with deionized water with an initial turbidity of 6.8NTU using a desktop spectrophotometer with a 10mm flow cell in the concentration range of $0-1 \mathrm{mg} / \mathrm{L}$

Figure 33: Linear correlation of Magnafloc LT27AG polymer in unfiltered Rideau River water diluted 1:1 with deionized water with an initial turbidity of 6.8NTU using an in-line spectrophotometer with a $4 \mathrm{~mm}$ flow cell in the concentration ranges of (a) $0-3 \mathrm{mg} / \mathrm{L}$ and (b) $0-1 \mathrm{mg} / \mathrm{L}$.....

Figure 34: Linear correlation of Magnafloc LT27AG polymer in filtered (0.45 $\mu \mathrm{m})$ Rideau River water diluted 1:1 with deionized water with an initial turbidity of 3.2NTU using a desktop spectrophotometer with a $10 \mathrm{~mm}$ flow cell in the concentration range of $0-1 \mathrm{mg} / \mathrm{L}$.

Figure 35: Linear correlation of Magnafloc LT27AG polymer in filtered $(0.45 \mu \mathrm{m})$ Rideau River water diluted 1:1 with deionized water with an initial turbidity of 3.2NTU using an in-line spectrophotometer with a $4 \mathrm{~mm}$ flow cell in the concentration ranges of (a) $0-3 \mathrm{mg} / \mathrm{L}$ and (b) $0-1 \mathrm{mg} / \mathrm{L}$

Figure 36: Absorbance spectra of Magnafloc LT27AG polymer in deionized water dosed with Actiflo $\Theta$ enhanced coagulation process chemicals using a desktop spectrophotometer with a $10 \mathrm{~mm}$ flow cell in the concentration ranges of (a) $0-3 \mathrm{mg} / \mathrm{L}$ and (b) $0-1 \mathrm{mg} / \mathrm{L}$.

Figure 37: Absorbance spectra of Magnafloc LT27AG polymer in deionized water dosed with Actiflo $\circledast$ enhanced coagulation process chemicals using an in-line spectrophotometer with a $4 \mathrm{~mm}$ flow cell in the concentration ranges of (a) $0-3 \mathrm{mg} / \mathrm{L}$ and (b) $0-1 \mathrm{mg} / \mathrm{L}$.

Figure 38: Absorbance spectra of Magnafloc LT27AG polymer in Rideau River (32.0 NTU) water dosed with Actiflo $\Theta$ enhanced coagulation process chemicals using a desktop spectrophotometer with a $10 \mathrm{~mm}$ flow cell in the concentration ranges of (a) $0-3 \mathrm{mg} / \mathrm{L}$ and (b) $0-1 \mathrm{mg} / \mathrm{L}$ 
Figure 39: Absorbance spectra of Magnafloc LT27AG polymer in Rideau River water (32.0 NTU) dosed with Actiflo $\Theta$ enhanced coagulation process chemicals using an in-line spectrophotometer with a $4 \mathrm{~mm}$

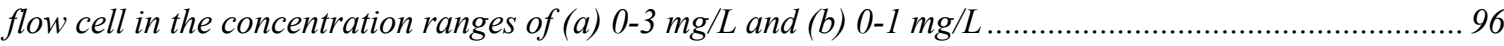

Figure 40: Absorbance spectra of (a) potassium permanganate and (b) PASS-10 coagulant in deionized water using a desktop spectrophotometer and a $10 \mathrm{~mm}$ flow cell

Figure 41: Absorbance spectra of (a) potassium permanganate and (b) PASS-10 coagulant in deionized water using a in-line spectrophotometer and a $4 \mathrm{~mm}$ flow cell.

Figure 42: Linear correlation of Magnafloc LT27AG polymer in deionized water dosed with Actiflo $\circledast$ enhanced coagulation process chemicals using a desktop spectrophotometer with a $10 \mathrm{~mm}$ flow cell in the concentration ranges of (a) $0-3 \mathrm{mg} / \mathrm{L}$ and (b) $0-1 \mathrm{mg} / \mathrm{L}$.

Figure 43: Linear correlation of Magnafloc LT27AG polymer in deionized water dosed with Actiflo $\Theta$ enhanced coagulation process chemicals using an in-line spectrophotometer with a $4 \mathrm{~mm}$ flow cell in the concentration ranges of (a) $0-3 \mathrm{mg} / \mathrm{L}$ and (b) $0-1 \mathrm{mg} / \mathrm{L}$.

Figure 44: Linear correlation of Magnafloc LT27AG polymer in Rideau River (32.0 NTU) water dosed with Actiflo $\Theta$ enhanced coagulation process chemicals using a desktop spectrophotometer with a $10 \mathrm{~mm}$ flow cell in the concentration ranges of (a) $0-3 \mathrm{mg} / \mathrm{L}$ and (b) $0-1 \mathrm{mg} / \mathrm{L}$.

Figure 45: Linear correlation of Magnafloc LT27AG polymer in Rideau River (32.0 NTU) water dosed with Actiflo $\circledast$ enhanced coagulation process chemicals using an in-line spectrophotometer with a $4 \mathrm{~mm}$ flow cell in the concentration ranges of (a) $0-3 \mathrm{mg} / \mathrm{L}$ and (b) $0-1 \mathrm{mg} / \mathrm{L}$.

Figure 46: Turbidity values corresponding to Magnafloc LT27AG polymer concentrations within the optimum dose range in the supernatant of a high turbidity (9172NTU) Rideau River water sample........ 108 Figure 47: Turbidity values corresponding to Magnafloc LT27AG polymer concentrations within the optimum dose range in the supernatant of a low turbidity (260 NTU) Rideau River water sample ........... 109 Figure 48: Turbidity values corresponding to Magnafloc LT27AG polymer concentrations within the optimum dose range in the supernatant of a high turbidity (9000 NTU) Rideau River water sample dosed with Actiflo $\circledast$ enhanced coagulation process chemicals.

Figure 49: UV-vis absorbance spectra of Magnafloc LT27AG polymer in a sample of high turbidity $(9172$ $N T U)$ Rideau River water using a (a) desktop spectrophotometer and a (b) in-line spectrophotometer for identifying the samples optimum polymer dose.

Figure 50: UV-vis absorbance spectra of Magnafloc LT27AG polymer in a sample of low turbidity (260 NTU) Rideau River water using a (a) desktop spectrophotometer and a (b) in-line spectrophotometer for identifying the samples optimum polymer dose...

Figure 51: UV-vis absorbance spectra of Magnafloc LT27AG polymer in a sample of high turbidity (9000 NTU) Rideau River water dosed with Actiflo enhanced coagulation process chemicals using a (a) desktop spectrophotometer and a (b) in-line spectrophotometer for identifying the samples optimum polymer dose

Figure 52: Linear correlation of Magnafloc LT27AG absorbance at 190nm in high turbidity (9172 NTU) Rideau River water using a (a) desktop spectrophotometer and a (b) in-line spectrophotometer for determination of the sample's optimum polymer dose.

Figure 53: Linear correlation of Magnafloc LT27AG absorbance at 190nm in low turbidity (260 NTU) Rideau River water using a (a) desktop spectrophotometer and a (b) in-line spectrophotometer for determination of the sample's optimum polymer dose.

Figure 54: Linear correlation of Magnafloc LT27AG absorbance at 190nm in high turbidity (9000 NTU)

Rideau River water dosed with Actiflo $®$ enhanced coagulation process chemicals using a (a) desktop spectrophotometer and a (b) in-line spectrophotometer for determination of the sample's optimum polymer dose. 118

Figure 55: Particle counts corresponding to Magnafloc LT27AG polymer concentrations within the optimum dose range in the supernatant of a high turbidity (9172 NTU) Rideau River water sample. 121 
Figure 56: Particle counts corresponding to Magnafloc LT27AG polymer concentrations within the optimum dose range in the supernatant of a low turbidity (260 NTU) Rideau River water sample...

Figure 57: Particle counts corresponding to Magnafloc LT27AG polymer concentrations within the optimum dose range in the supernatant of a high turbidity (9000 NTU) Rideau River water sample dosed

with Actiflo $®$ enhanced coagulation process chemicals

Figure 58: Turbidity values corresponding to Hydrex 3661 polymer concentrations within the optimum dose range in a high turbidity (4426 NTU) Rideau River water sample supernatant......

Figure 59: Turbidity values corresponding to Hydrex 3661 polymer concentrations within the optimum dose range in a low turbidity (546 NTU) Rideau River water sample supernatant.

Figure 60: UV-vis absorbance spectra of Hydrex 3661 polymer in a sample of high turbidity (4426 NTU)

Rideau River water using a (a) desktop spectrophotometer and a (b) in-line spectrophotometer for identifying the samples optimum polymer dose

Figure 61: UV-vis absorbance spectra of Hydrex 3661 polymer in a sample of low turbidity (546 NTU)

Rideau River water using a (a) desktop spectrophotometer and a (b) in-line spectrophotometer for identifying the samples optimum polymer dose

Figure 62: Linear correlation of Hydrex 3661 polymer absorbance at 190nm in high turbidity (4426 NTU)

Rideau River water using a (a) desktop spectrophotometer and a (b) in-line spectrophotometer for

determination of the sample's optimum polymer dose.....

Figure 63: Linear correlation of Hydrex 3661polymer absorbance at 190nm in low turbidity (546 NTU)

Rideau River water using a (a) desktop spectrophotometer and a (b) in-line spectrophotometer for determination of the sample's optimum polymer dose.

Figure 64: Particle counts corresponding to Hydrex 3661 polymer concentrations within the optimum dose range in a high turbidity (4426 NTU) Rideau River water sample supernatant

Figure 65: Particle counts corresponding to Hydrex 3661 polymer concentrations within the optimum dose range in a low turbidity (546 NTU) Rideau River water sample supernatant 


\section{CHAPTER 1: INTRODUCTION}

The majority of water sources utilized for drinking water contain some concentration of dissolved impurities comprised of minerals, gases, and organic compounds that alter the chemical (biological and chemical oxygen demand, $\mathrm{pH}$, total organic carbon), physical (turbidity, temperature, colour) and biological characteristics of water (1). Safe drinking water is essential to human health, which is why every drinking water source undergoes some form of purification prior to consumption. Various treatment methods are employed to render water safe and appealing to drink, which are dependent on the above listed characteristics of the water source. A major issue regarding the treatment of surface waters is the substantial seasonal variation in the physical characteristic of turbidity (2).

Of all the methods used for the production of safe drinking water; the application of chemical coagulants such as ferric chloride, and both alum and polyelectrolyte polymers have proven to increase the efficiency of suspended solid (colloid) separation from water matrices, decreasing turbidity (3). Drinking water treatment systems frequently make use of a variety of different polymers, typically polyelectrolytes, during the coagulation and flocculation stages of the treatment process. These polymers act as either primary coagulants or flocculant aids to improve efficiency of the coagulation and flocculation stages by lowering coagulant dose, decreasing the ionic load of the treated water, improving the settling of slow-settling flocs in low-temperatures, improving settleability and toughness of flocs, decreasing operating costs, and increasing treatment facility 
capacity (4) (5) (6). The polymers bridge the microfloc formed by coagulation, agglomerating smaller particles into larger ones (7).

Polymers function most effectively within an optimum dose range, which is dependent on the properties of the water sample in which they will be used. Dosing polymer at a concentration less than optimum (under-dosing) renders the chemical ineffective, as there is not an adequate concentration of polymer present with respect to the concentration of suspended colloids in the water (5). Dosing polymer at a concentration greater than optimum (overdosing) leads to the production of residual polymer left suspended in solution post-treatment; which constitutes any polymer that was not adsorbed onto particulate matter during the coagulation-flocculation process, and often contains monomer units as well as other contaminants that may have been present in the original polymer.

The presence of residual polymer has proven to cause many operational and environmental issues. The viscous nature of dissolved polymer has been shown to cause issues with the clogging of filter beds and filter head loss in wastewater sludge applications, which is also suspected to affect water treatment applications (8). In addition, overdosing can lead to the restabilization of flocs, breaking the flocs, and allowing particulates and contaminants to re-enter the water matrix (3). Overdosing also leads to a larger sludge yield, which increases disposal costs and is undesirable. Furthermore, polymer addition can be a very expensive addition to municipal drinking water treatment if not dosed correctly. In terms of environmental concerns, polyelectrolyte products used for water treatment applications often contain detectable amounts of certain contaminants such as monomer and unreacted 
chemicals used to form the monomer, which are toxic to humans and fauna (9). The monomer units have been shown to be more toxic than the polymers themselves, thus the World Health Organization (WHO) has set maximum dosage limits for polymers in water treatment that are usually $<50 \mathrm{mg} / \mathrm{L}$ for polydiallyldimethylammonium chloride (PDADMAC) polymer, $<20 \mathrm{mg} / \mathrm{L}$ epichlorohydrin $(\mathrm{ECH} / \mathrm{DMA})$ polymers and $<1.0 \mathrm{mg} / \mathrm{L}$ for polyacrylamide (PAM) polyelectrolytes in the USA (10).

The detection of residual polymer and the optimization of polymer dose continues to be a major challenge for drinking water treatment facilities. Differences in source water characteristics such as; $\mathrm{pH}$, temperature, coagulant type, concentration of suspended particles present, as well as the upstream treatment systems can alter the optimum dosage concentration significantly (11). The source, composition charge, particle size, particle shape, and density of the suspended particles present in the source water also vary considerably; with the correct dosage of polymer being contingent upon these variables. The majority of drinking water treatment plants tend to use operators for the manual adjustment of polymer dose based on major changes noticed in settleability or seasonal turbidity changes in source water (12). The optimum dose is most often pre-determined using a jar test method seasonally. This method is limited seeing that is only dependant on supernatant turbidity, which reaches a minimum at optimum dose. Although this is a good approximation, the turbidity does not vary beyond optimum polymer dose and does not give an indication of how much polymer is overdosed, often leading to overdosing. This results in higher polymer costs, ineffective coagulation-flocculation, and an excess release of residual polymer downstream, which is then discharged into the environment. 
There is a clear need for the development of a reliable system that can sensitively produce real-time measurements of residual polymer dose during drinking water treatment, and ideally develop a feedforward or feedback control loop for the optimization of polymer dose in-line. Some of the methods that have been suggested for this purpose include the development of a feedforward control system that optimizes coagulant dose based on continuous measurement of raw water quality through parameters such as turbidity (13) (14), conductivity, NOM removal, and/or surface charge (15). The development of a feedback control system that optimizes coagulant dose based on raw or dosed water quality is another method that has been studied; with the use of streaming current detection (SCD) being most common for this application (16) (17) (18). The use of online sensors that (19)analyze the size and shape of floc formation have also been applied in research, such as the use of a photometric dispersion analyzer (PDA) that measures turbidity and turbidity fluctuations after coagulant addition via a fiber-optical monitor (20) (21); but have not been tested in practice. Other very sensitive methods of measuring polymer dose in water treatment applications in a laboratory setting include high-performance liquid chromatography (HPLC) and electron-gas capture chromatography; however, these methods are impractical for in-line use.

Although these methods have been studied extensively, they did not find successful applications in drinking water treatment facilities due to a variety of reasons. The complexity and capacity required to carry out the method, the lack of instruments designed for full-scale application, the fluctuation of source water characteristics impacting the sensitivity of the method, and lastly the difficulties with replicability of results in the field 
have led to little progress in further development of these methods. Moreover, the bulk of the research available for these methods is based on wastewater applications. In drinking water applications, particulate matter is present in far lower concentrations, and there are very few approaches that analyze finished drinking water parameters for real-time control purposes (15).

Ultraviolet-visible light (UV-vis) spectroscopy is a method that has been proposed for the measurement of polyacrylamide polymers in water and wastewater applications using an in-line spectrophotometer (22). Al Momani and Örmeci have conducted research on this method, with results indicating that the concentration of residual polyacrylamide polymers in water and wastewater matrices can be correlated to the absorbance of the dosed water at a wavelength of around 190nm, with quite low detection limits $(<0.1 \mathrm{mg} / \mathrm{L})$. Further research by Al Momani and Örmeci investigated the optimization of sludge conditioning and dewatering based on residual polymer in the dewatering supernatant through the use of UV-vis spectrophotometry (23). The results of this study indicated that the optimum polymer dose for sludge dewatering could be correlated to the lowest filtrate absorbance at or around $191.5 \mathrm{~nm}$. Although these results demonstrate that UV-vis spectroscopy is a viable method for the in-line determination of optimum polymer dose, little work has been done on the application of this method for drinking water treatment. 


\section{Research Objectives}

The principle objectives of this research are to improve the sensitivity of UV-vis spectroscopy as a method of monitoring and optimizing polymer dose in drinking water treatment applications, and test whether it can confidently measure these low concentrations in the presence of other constituents in-line and real time.

Firstly, this study explores the effect of path length, particles, and additional drinking water treatment chemicals present in water on the sensitivity of UV-vis spectroscopy as a method for the detection of residual polymer in drinking water treatment applications. This is done to conclude whether the polymer detection sensitivity can be improved by manipulating these parameters, improving the linear correlation between residual polymer concentration and absorbance, as well as determine where the unit would be best suited for in-line monitoring during drinking water treatment. Figure 1 below illustrates the potential for residual polymer monitoring using this method in a drinking water treatment application.

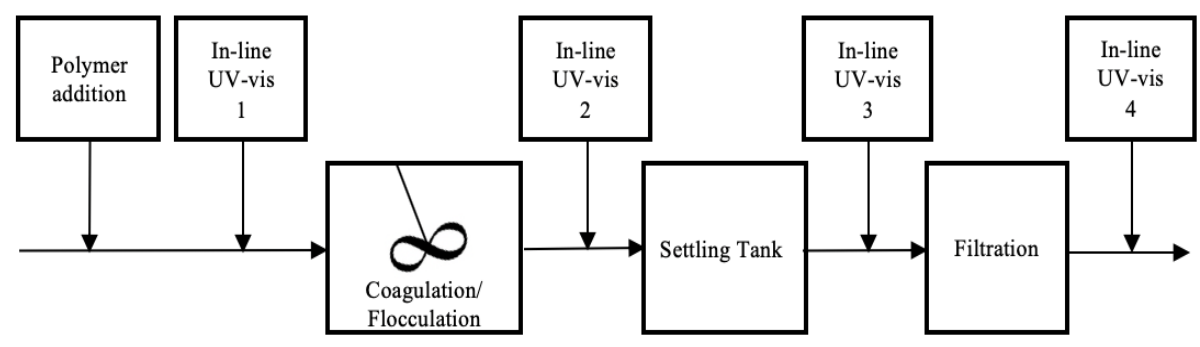

Figure 1: UV-vis spectroscopy as a tool for the in-line and real time monitoring of residual polymer in drinking water treatment applications schematic

Secondly, this study aims to determine whether UV-vis spectroscopy has potential as a tool for the optimization of polymer dose in-line and real time during drinking water treatment. 
The relationship between absorbance and residual polymer concentration post-settling in the optimum dose range is explored, and the potential to create a feedback control loop using this relationship in drinking water treatment applications is determined. Figure 2 below illustrates this optimization application in a drinking water treatment settling.

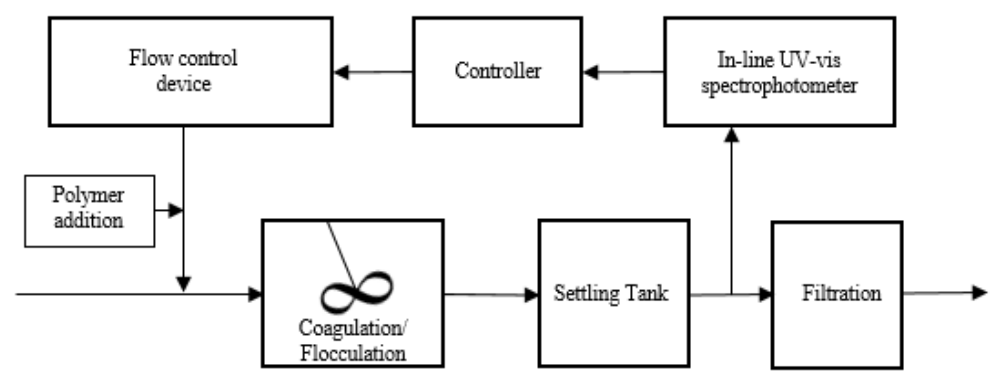

Figure 2: UV-vis spectroscopy as a tool for the in-line and real time optimization of residual polymer in drinking water treatment applications schematic

In order to study these objectives, this research has been divided into four main experimental phases:

\section{Phase 1: Effect of path length on polymer detection using $U V$-vis spectroscopy}

The first phase of the experiment was designed to compare the effect of path length on polymer detection sensitivity using both an in-line and desktop spectrophotometer. This phase also allowed for a comparison in functionality and sensitivity between both the inline and desktop spectrophotometers. In addition, effect of polymer dose range was also accounted for. 
The main purpose behind comparing different path lengths was to determine the effect this alteration would have on the sensitivity of UV-vis spectroscopy as a method for the detection of residual polymer in drinking water sample matrices. The Beer-Lambert law (equation 5) is the governing principle behind UV-vis spectroscopy and assumes that the absorbance and the path length of the sample cuvette are directly proportional. Increasing the path length of the sample cuvette allows the incident light more opportunity to interact with the molecules of interest present in the sample, thus increasing the final absorbance reading and enabling a higher detection sensitivity. Contrastingly, a decrease in path length has also been shown to yield more sensitive result in certain applications, due to the theory that a longer path length allows for more light scattering and a decrease in sensitivity, especially for solutions with very low concentrations (24). In addition, a shorter path length typically reduces the absorbance (increasing the transmittance) hence reducing the incident light required to achieve a reliable result. This removes the need to dilute the sample, which is often necessary to achieve an absorbance within the working range of the unit.

An in-line spectrophotometer was used to compare the effect of altering the path length between $2 \mathrm{~mm}, 4 \mathrm{~mm}$ and $8 \mathrm{~mm}$ for an anionic polyelectrolyte polymer used for potable water treatment. These results were then compared to those produced using a desktop spectrophotometer with a typical $1 \mathrm{~cm}$ path length. When altering the path length, the absorbance results are normalized to be comparable to that of a $1 \mathrm{~cm}$ path length, thus changing the working range with regard to each different flow cell. Using the in-line spectrophotometer, the $2 \mathrm{~mm}$ path length has a working range of 0-6.5 A.U., the $4 \mathrm{~mm}$ path length has a working range of 0-3.25 A.U., and the $8 \mathrm{~mm}$ path length has a working range 
of 0-1.6 A.U. Using the results from this phase, an optimum path length could be determined for the polymer in question for the subsequent phases.

\section{Phase 2: Effect of filtration and dilution on polymer detection using $U V$-vis spectroscopy}

The second phase of the experiment was designed to analyze the effect of particles present in water on the sensitivity of UV-vis spectroscopy as a method of measuring residual polymer in drinking water treatment applications. This was done by analyzing the effects of filtration and dilution on polymer detection sensitivity, using both an in-line and desktop spectrophotometer on the relationship between polymer concentration and sample absorbance.

Particles present in the solution can often interfere with the accuracy of the UV-vis absorbance reading when trying to identify a particular constituent, as they can absorb or scatter the incident light. Comparing results for samples with particles (unfiltered) and without particles (filtered) using both a $1 \mathrm{~cm}$ path length and a shorter path length was carried out to observe the effect that the path length had on UV-vis absorbance sensitivity in the presence of particles. A shorter path length yields less opportunity for the particles to interact with the incident light, as is thought to produce more sensitive results. Dilution of samples was also analyzed to observe the same effect when the particles present were reduced in concentration, rather than eliminated completely. This was performed on both an in-line and desktop spectrophotometer at both high and low polymer dose ranges for comparison purposes. This was done using the optimum path length for the in-line UV-vis spectrophotometer determined from phase one. 
Phase 3: Effect of Actiflo@ treatment process on polymer detection using UV-Vis spectroscopy

The third phase of the experiment was designed to determine the effect of drinking water treatment process chemicals on the sensitivity of UV-vis spectroscopy as a method of detecting residual polymer post drinking water treatment and determine whether or not the method would be viable in a full-scale drinking water treatment setting. This was done by analyzing the effects of the chemical additives used during the Actiflo ${ }^{\circledR}$ coagulation and decantation treatment process, on the linear relationship between polymer concentration and sample absorbance.

Similarly to particles present in solution, additional treatment chemicals can often interfere with the accuracy of the UV-vis absorbance reading when trying to identify a particular constituent, as they can absorb or scatter the incident light. Comparing results for samples with and without additional. treatment chemicals present using both a $1 \mathrm{~cm}$ path length and a shorter path length was carried out to observe the effect that the path length had on UVvis absorbance sensitivity in the presence of particles. A shorter path length yields less opportunity for the chemicals to interact with the incident light, as is thought to produce more sensitive results. This was performed on both an in-line and desktop spectrophotometer at both high and low polymer dose ranges for comparison purposes. This was again done using the optimum path length for the in-line UV-vis spectrophotometer determined from phase one. 


\section{Phase 4: Determination of optimum polymer dose using $U V$-vis spectroscopy}

The fourth phase of the experiment was designed to determine the viability of UV-vis spectroscopy as an in-line real time method for the determination of optimum polymer dose for a given drinking water source. As previously mentioned, optimum polymer dose in drinking water treatment is typically identified using a jar test. If polymer is dosed less than optimally, it does not function effectively and the sample will contain a higher number of non-settled particles, contributing to the supernatant turbidity. As optimum dose is approached, there are limited particles left in the solution and the supernatant turbidity will decrease until it reaches a minimum once optimum dose is reached. Beyond this dose, turbidity will remain at a constant minimum.

Al Momani and Örmeci demonstrated in a previous study that polymer, when dosed optimally, will produce a minimum absorbance in sludge centrate applications using UVvis spectroscopy. Similarly to turbidity, when polymer is underdosed it does not function effectively and the sample will contain a higher number of non-settled particles, contributing to the supernatant absorbance. As polymer dose is increased and optimum dose is approached, the polymer will incrementally remove more particulate matter present in the sample and the supernatant absorbance will decrease until it reaches a minimum value at the optimum dose. Beyond this dose, any excess polymer added remains suspended in solution as residual, which contributes to an increase in absorbance. This is an advantage of using UV-vis spectroscopy as opposed to a traditional jar test for optimum dose determination, as it gives a better indication of polymer overdosing through absorbance increase. 
This theory was tested using potable water samples of varying turbidity using a cationic and an anionic polymer, as well as with Actiflo ${ }^{\circledR}$ chemical addition. This was done to observe the effects of turbidity variance (number of particles present), polymer type, and chemical additives on the sensitivity of this method for optimum dose determination using both an in-line and desktop spectrophotometer. The optimum dose results based on absorbance measurements were compared with preliminary settling test and turbidity measurements, as well as particle count data for each sample to observe if the minimum absorbance corresponded to the same polymer dose as the minimum turbidity and particle concentration.

\subsection{Thesis layout}

\section{Chapter 1}

Chapter one introduces the topic of the thesis, highlights the need for the study and presents the research objectives of the study.

\section{Chapter 2}

Chapter two provides a literature review outlining relevant background information pertaining to the topic of the thesis in order to give the reader the capacity to follow the thesis.

\section{Chapter 3}

Chapter three provides a description of the materials and methods used to carry out the experiments performed during this thesis in order to study the outlined research objectives. 


\section{Chapter 4}

Chapter four presents the results and analysis of all four experimental phases of the study as outlined above. Absorbance spectra are presented for all datasets to demonstrate the relationship between absorbance trends and polymer dose. In addition, plots of the linear relationship between polymer concentration and absorbance at $190 \mathrm{~nm}$ are also included to compare the linearity of this relationship between samples. The results of the fourth experimental phase also present settling test results, turbidity results, and particle concentration results for all datasets completed during this experimental phase. Chapter four also discusses other analytical methods that have been studied for the determination of optimum polymer dose in water treatment applications, and how these methods compare to UV-vis spectroscopy in terms of viability, sensitivity and applicability.

\section{Chapter 5}

Chapter five provides concluding statements based on the findings of this study, as well as recommendations for future work.

It is our hope that the work done to accomplish the principle objective of this study will contribute to the overall improvement of polymer dosing and efficiency in drinking water treatment applications. The development of a simple, sensitive and direct method for the in-line determination of optimum polymer dose in drinking water treatment applications will aid facilities not only in improving treatment performance; but also in reducing and ideally eliminating the release of toxic polyelectrolyte polymers into the environment. 


\section{CHAPTER 2: LITERATURE REVIEW}

\subsection{Applications of polymers in drinking water treatment}

Drinking water treatment processes are of the most fundamental industrial processes carried out in anthropologically in our world today. These processes exist to provide us with readily available water that is safe to drink, free of any unpleasant tastes or odors, cost-effective to produce, free of viruses and pathogens, and ultimately safe for public health (25) (26). Although the drinking water treatment process can vary depending on the source water, the basic unit processes remain generally the same. An overview of the typical drinking water unit operations is depicted below in Figure 3. Firstly, source water, either surface or groundwater, is subjected to a coagulation and flocculation stage, which is typically considered a chemical treatment. Afterwards, the water undergoes a sedimentation stage followed by some form of filtration, both of which are considered physical treatments. Finally, the water is disinfected by either physical or chemical means to ensure it is free of any residual bacteria, viruses and/or protozoa before entering the distribution system (26) (27).

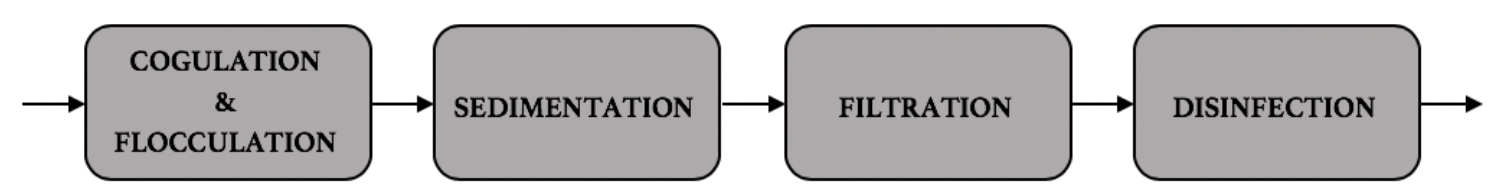

Figure 3: General drinking water treatment processes

During the coagulation and/or flocculation stages of drinking water treatment, watersoluble polymers are sometimes added as primary coagulants, or as coagulant/flocculant 
aids (4) (5) (6). The principle function of coagulation is to reduce the repulsive potential of the electrical double layer of colloids suspended in the water source in such a manner that micro-particles can begin formation. These micro-particles can then collide with one another and form larger structures known as flocs in the subsequent flocculation stage (28). The overall goal of the coagulation and flocculation stage in drinking water treatment is to reduce turbidity, remove unpleasant colour and remove pathogens by pulling the particles together and increasing their size and weight; allowing them to be settled out or filtered out more effectively (28). Polymers have been shown to improve the coagulation and flocculation stages by lowering coagulant dose, decreasing the ionic load of the treated water, improving the settling of slow-settling flocs in low-temperatures, improving settleability and toughness of flocs, decreasing operating costs and increasing treatment facility capacity (5) (4). The polymers chosen for this purpose are typically synthetic in nature, although there are a number of organic products that are also of interest for this application. These polymers can be broadly categorized as polyelectrolytes (either anionic, cationic, or non-ionic) (5). The polymer molecules themselves are constructed of monomer units linked together as either linear chains or branched three-dimensional networks. The majority of those used in water treatment applications are linear chain structures with a minimum degree of branching (3). The number of repeating monomers in the chain known as the degree of polymerization. The product of the degree of polymerization and the molecular weight (MW) of the monomer yields the MW of the polymer. In general, low MW cationic polymers are used as coagulants or coagulant aids; whereas high molecular weight polyelectrolytes or non-ionic polymers are used as flocculants (3). 


\subsection{Mechanisms of polymer function}

In drinking water treatment applications, there are two main mechanisms of coagulation by which a polymer can function: polymer bridging and charge neutralization (29). Both of these mechanisms are strongly dependant on the adsorption of the polymer onto the suspended colloidal surface. This adsorption can only occur if there is some sort of affinity between the colloidal surface and the polymer chain that is greater than the loss of entropy attributed to polymer adsorption (28). This adsorption can occur through several different methods, the main of which being a combination of electrostatic interaction and van der Waals attraction (5) (4).

Electrostatic interaction occurs when polyelectrolytes with a charge opposite to that of the colloid surface adsorb by simple means of electrostatic attraction. Most colloidal particles in aqueous suspension are negatively charged, depending on the chemistry of the solution. A colloidal dispersion, however, does not have any charge regardless of the charge attributed to the particles themselves. In order for this electroneutrality to exist, the colloidal charge must be counterbalanced by counter ions present in the aqueous solution. This compact, fixed layer of counter ions surrounding the colloid is known as the Stern layer and is depicted in Figure 4 below. Surrounding this Stern layer, a more diffused layer of counter ions exists (Gouy-Chapman layer). Together, the Stern layer and the diffuse layer form what is called the electrical double layer. 


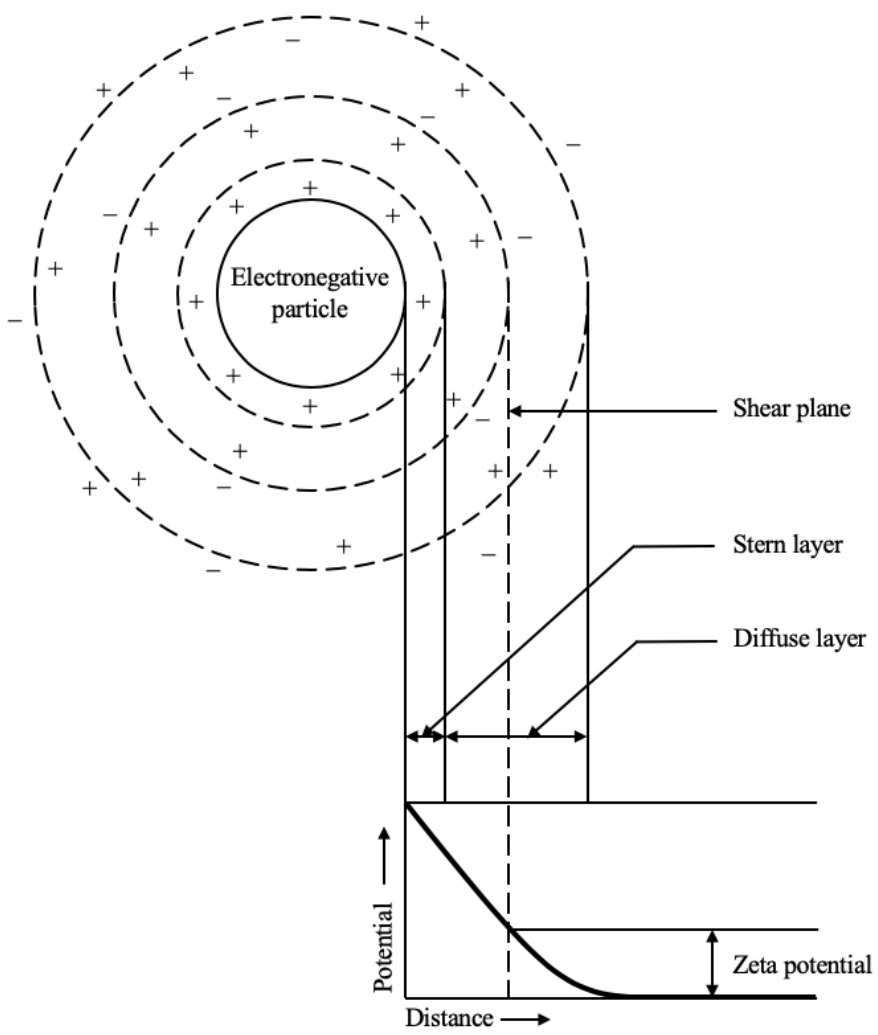

Figure 4: A negative colloidal particle with its electrostatic field demonstrating the electrical double layer (Forbes, E., Chryss, A., Clays in the Minerals Processing Value Chain, 2017)

A shear plane exists between the Stern layer and the diffuse layer and is thought to be located a minute distance beyond the Stern layer that encloses the volume of bound water that moves with the particle. The electrostatic potential that exists between this bound water and the bulk liquid surrounding it is said to exist at this shear plane and is known as the zeta potential $(\xi)$. The zeta potential is of particular importance, as it is the only measurable potential and provides a good approximation of the unmeasurable surface potential. Moving outwardly from the shear plane into the diffuse layer, the zeta potential decreases (3). The electrical double layer model allows for the determination of the stability of a colloidal solution. The interaction between two similarly charged colloidal particles is prevented as their diffuse layers overlap and their Stern layers repel one another, preventing 
aggregation. The magnitude of this repulsive force is dependent on the surface potential of the particles, or the zeta potential (3).

Polyelectrolytes with a higher MW will be more effective for absorption by electrostatic interaction as they offer more ionic groups for attraction, however even low MW polymers are capable of at minimum neutralizing the particles surface charge (5). In drinking water treatment applications, natural organic matter (NOM) is most often negatively charged, and therefore cationic polyelectrolytes are often preferred in application. Polymer adsorption can also occur through hydrogen bonding and ion binding. Certain polymers, such as polyacrylamide (PAM), have the capability to form hydrogen (H)-bonds with particles that have suitable bonding sites. For example, silica oxides have surface hydroxyl groups that are capable of forming H-bonds with the amide groups present in a PAM molecule (30). Ion binding occurs in instances where there is a large concentration of divalent metal ions and $\mathrm{Ca}^{+}$. It is theorized that these ions act as 'bridges' between anionic polymers and negatively charged particles, allowing for ion binding to occur despite electrostatic repulsion (31). Regardless of the adsorption mechanism, Figure 5 below depicts the general accepted model for an adsorbed polymer consists of three assumed segment categories:

- trains - polymer segments directly attached to the particle surface,

- loops - polymer segments erecting between trains along the particle surface,

- tails - polymer segment ends that protrude from the particle surface into solution. 


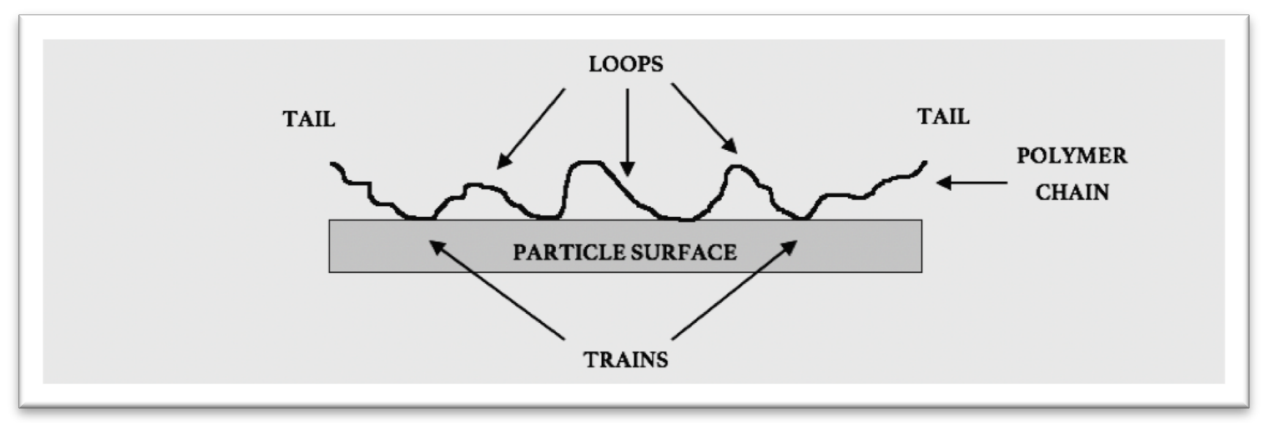

Figure 5: adsorbed polymer model

\subsubsection{Polymer bridging}

The first main coagulation mechanism, as previously mentioned, is known as polymer bridging. Bridging occurs when the polymer adsorbs to the particle in such a way that allows for a sufficient number of tails and loops to attach to those alike surrounding it. A schematic of this mechanism is illustrated below in Figure 6.

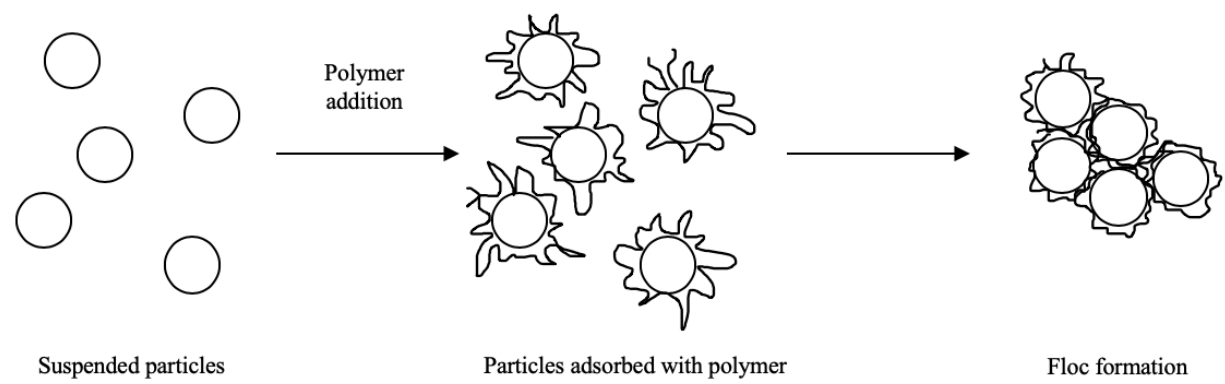

Figure 6: Polymer bringing mechanism schematic (Singh, H. et al., High-technology materials based on modified polysaccharides, 2009)

In order for bridging to occur, there must be an adequate number of unoccupied sites on the particle adjacent to that of another particle with an adsorbed polymer. If the particle is too highly covered with polymer, it is considered restablished and bridging cannot occur. Alternatively, if there is insufficient polymer coverage then bridging also becomes impossible since the proper contingence cannot occur, which infers the idea of an optimum 
polymer dose for maximum bridging. Early studies on optimum dose suggested that $50 \%$ fractional surface coverage $(\theta)$ corresponds to the optimum polymer dose by means of the following equation: $\theta(1-\theta)(32)$. Due to difficulties accurately measuring particle surface coverage, studies that are more modern have shown that the optimum dosage occurs when essentially the entire polymer is adsorbed, implying that the optimum dose be proportional to aqueous particle concentration. This value is typically $1.0 \mathrm{mg}$ of polymer per gram of suspended solids (5). High MW polyelectrolytes with a variable charge density (CD) have proven to be most effective for bridging applications, since they have less bulky branch groups to hinder available sites, but also have longer chains to allow for ample bridging. With regard to $\mathrm{CD}$, a cationic polyelectrolyte with roughly $30 \%$ anionic groups is most effective as the anionic groups allow for a degree of electrostatic repulsion, expanding the chains and encouraging bridging.

\subsubsection{Charge neutralization}

In most water matrices, suspended impurities are negatively charged, making cationic polymers most effective due to their electrostatic adsorption ability facilitating their attraction onto the particles. This adsorption causes the particles to take on a neutral charge, or even undergo a charge reversal. This has been theorized as charge neutralization, the idea that particles are able to coagulate and flocculate by merely reducing the electrostatic repulsion between them through polymer addition (5). Typically, cationic polyelectrolytes with a low MW and high CD are best suited for this application in water treatment, as they are able to neutralize the particle charge and adsorb in a flatter configuration on the particle surface due to the lack of bulky groups along their chain (29). Another extension of charge 
neutralization that can occur is the electrostatic patch mechanism. The basic concept of this mechanism is that if a high $\mathrm{CD}$ cationic polymer adsorbs onto a low $\mathrm{CD}$ anionic particle, the distance between negatively charged sites on the particle may be so far that the charged segments of the polymer cannot physically reach them all and, in turn, cannot attain overall neutrality. This then creates 'patches' of positive charge over the particle surface while leaving some uncovered negatively charged sites, allowing for particle attachment to occur via electrostatic attraction between the two oppositely charged sites.

\subsection{Operational and environmental disadvantages of polymer in drinking water} treatment

Although the use of polymer in drinking water treatment applications does have many benefits when implemented properly, there are also some important operational and environmental disadvantages to consider. Polymer toxicity is one factor that has caused concern in recent years, especially with regard to their use in drinking water treatment. In general, most anionic and non-ionic polyelectrolytes are of low toxicity to humans and aquatic life. However, most cationic polyelectrolytes are acutely toxic to humans, animals and aquatic flora and fauna when they are present within the range of $0.1-1.0 . \mathrm{mg} / \mathrm{L}(33)$. In addition, polyelectrolyte products used for water treatment applications often contain detectable amounts of certain contaminants such as monomer and unreacted chemicals used to form the monomer (9). The monomer units have proven to be more toxic than the polymers themselves because their smaller molecular structure allows them to be more readily adsorbed into the gastrointestinal tract and lymphoid tissues, especially with regard to acrylamide products (34). Although the monomer poses much more of a risk than the 
polymers themselves, measuring individual monomers post-water treatment can be very difficult, and it is therefore best practice to consider any toxic hazard on the basis of the applied dose of the polymer. For this reason, the World Health Organization (WHO) has set maximum dosage limits for polymers in water treatment that are usually $<50 \mathrm{mg} / \mathrm{L}$ for Polydiallyldimethylammonium chloride (PDADMAC) polymer, $\quad<20 \mathrm{mg} / \mathrm{L}$ Epichlorohydrin (ECH/DMA) polymers and $<1.0 \mathrm{mg} / \mathrm{L}$ for polyacrylamide (PAM) polyelectrolytes in the USA (10).

Dosing the correct volume of polymer for a given water treatment application is an important factor in the process not only to meet regulation, but also for operational reasons. Under conditions where a polymer has been overdosed, particles may become restabilized by the adsorption of excess polymer. When the concentration of polymer is higher than that needed for flocculation, the particle surface becomes entirely covered by adsorbed polymer, leaving little to no available opportunity for the bridging or charge neutralization mechanisms to occur (3). This then results in not only poor flocculation performance, but also an excess of residual polymer in the water. Residual polymer constitutes any polymer that was not adsorbed onto suspended colloidal matter during the coagulation/flocculation process, and often contains monomer units as well as other contaminants that may have been present in the original polymer (5).

An excess in residual polymer can also cause many operational issues downstream, as well as toxicity issues upon release (35). As filtration most often follows polymer addition, excess residual polymer has proven to cause issues with the clogging of filter beds and 
filter head loss in wastewater sludge applications, which is also suspected to affect water treatment applications (8). One of the most widely used polyelectrolyes in drinking water treatment applications is polyacrylamide (PAM), which degrades into its acrylamide monomer of higher toxicity if left in aqueous solution. The US EPA limit for PAM in drinking water treatment applications is $1.0 \mathrm{mg} / \mathrm{L}$. At a monomer content of $0.05 \%$, this corresponds to a maximum theoretical concentration of $0.5 \mu \mathrm{g} / 1$ of the monomer in water. Practical concentrations may be lower by a factor of $2-3$. This applies to the anionic and non-ionic polyacrylamides, but residual levels from cationic polyacrylamides may be higher (10).

In addition to this, as much as an under-dose of polymer can affect the flocculation quality, an overdose can as well. As previously mentioned, restabilization of flocculated particles can occur when the polymer dose exceeds its optimum value, breaking the flocs and allowing particulates and contaminants to re-enter the water matrix (3). Overdosing also leads to a larger sludge yield, which increases disposal costs and is undesirable. Furthermore, polymer addition can be a very expensive addition to municipal drinking water treatment if not dosed correctly. BASF Inc., the manufacturer of the Magnafloc polymer analyzed in this study, reported a unit price of $1.36 \mathrm{USD}-1.62 \mathrm{USD} / \mathrm{kg}$ polymer in 2016. Although the savings a plant achieves in additional chemical use and filter backwash reduction are high, they typically will only outweigh the added cost of the polymer if the polymer is dosed optimally. In example, a water treatment plant in South Africa reported a $30 \%$ decrease in unit cost upon switching from alum to a polymeric coagulant, despite a $10 \%$ yearly inflation rate (36). 


\subsection{Current Polymer Dose Monitoring Systems in Drinking Water Treatment}

It is apparent that a quick and feasible method for monitoring the dosage of polymer is a vital need for water treatment facilities in order to ensure they are operating at optimal conditions, minimizing the release of residual polymer, and abiding by the set dosage limits. As it currently stands, many municipal water treatment facilities do not have any means of monitoring their polymer dosages on site, leading to operators frequently overdosing (31). There are a number of analytical methods that have been proven successful for the quantitative determination of polyelectrolytes in water, however they are typically quite extensive, time consuming and require specific skill capacities and laboratory access. A few of the most common methods used in practice include gas chromatography (GC), high-performance liquid chromatography (HPLC), mass spectrometry (MS) or a combination of these methods (22). Aside from these separation methods, a few other possibilities have been explored for the quantitative determination of polymers in water treatment, the main of which are summarized in Table 1. All of the below mentioned monitoring techniques are laboratory based aside from turbidimetry, which has the potential to be executed in-line however is not sensitive enough for the accurate detection of residual polymer in drinking water treatment applications due to the very low turbidity of drinking water sources and very low polymer dose concentrations. 
Table 1: Current methods for polymer monitoring in drinking water treatment applications

Polymer monitoring system Description

\begin{tabular}{|c|c|c|}
\hline 1 & Colloid titration & $\begin{array}{l}\text { Based on the titration of a cationic polyelectrolyte with } \\
\text { an anionic polyelectrolyte (or vice versa) in the } \\
\text { presence of an endpoint indicator ( } 37 \text { ). This method is } \\
\text { not very sensitive, can be easily affected by other } \\
\text { electrolytes in solution, and is not very practical to } \\
\text { perform in-line in an industrial setting. }\end{array}$ \\
\hline 2 & $\begin{array}{l}\text { Streaming current } \\
\text { detection (SCD) }\end{array}$ & $\begin{array}{l}\text { Streaming current is defined as a measurable electric } \\
\text { current that is generated by a mass water, which is } \\
\text { forced to flow past a network of fixed shear plane } \\
\text { surfaces. The measurable current is proportional to the } \\
\text { charge remaining after polymer addition. This allows } \\
\text { for the monitoring of the net residual charge balance of } \\
\text { the water, the positive charge representing the } \\
\text { coagulant and the negative charge representing the } \\
\text { suspended solids. Following the reduction of the } \\
\text { negative surface charge on the particles as polymer is } \\
\text { added, the optimum dose can be correlated with charge } \\
\text { neutrality (38). }\end{array}$ \\
\hline 3 & Turbidimetry & $\begin{array}{l}\text { There are a few different turbidimetric methods used for } \\
\text { polymer detection in solution, however each begin with } \\
\text { the addition of a reagent that reacts with the polymer to } \\
\text { produce an insoluble complex. This complex most often } \\
\text { remains suspended in solution and is measured by light } \\
\text { scattering, a turbidimeter or spectrophotometer. These } \\
\text { methods require large sample volumes and are of } \\
\text { limited use for the detection of acrylamide polymers in } \\
\text { brine solutions where interactions with sodium }\end{array}$ \\
\hline
\end{tabular}




\begin{tabular}{|c|c|c|}
\hline & & $\begin{array}{l}\text { chloride, iron, calcium and anionic surfactants cause } \\
\text { detection interference (37). }\end{array}$ \\
\hline 4 & Rheology/Viscosity & $\begin{array}{l}\text { Rheological characteristics of the solution have been } \\
\text { shown to also have the capacity to measure polymer } \\
\text { concentration. One method that has been studied } \\
\text { requires that the solution viscosity vary proportionally } \\
\text { with polymer concentration. These methods lack } \\
\text { practicability in practice seeing that any change in } \\
\text { temperature, ionic strength, polymer degree of } \\
\text { hydrolysis, pH, MW distribution or shear rate will have } \\
\text { some impact on the solutions overall viscosity. This } \\
\text { makes isolating the polymers effects on solution } \\
\text { viscosity essentially impossible in non-ideal conditions } \\
\text { (37). }\end{array}$ \\
\hline 5 & $\begin{array}{l}\text { Size exclusion } \\
\text { chromatography (SEC) }\end{array}$ & $\begin{array}{l}\text { SEC achieves a measurement by separating the polymer } \\
\text { from low MW impurities though a filtration column } \\
\text { with a specified pore size. This separated solution can } \\
\text { then be further analyzed by ultraviolet (UV) or } \\
\text { refractive index (RI) detectors, RI being more sensitive } \\
\text { than UV. This method is capable of measuring any UV } \\
\text { absorbing polymer in contaminated solutions, however } \\
\text { its sensitivity is greatly affected by the polymer's } \\
\text { degree of hydrolysis, as well as any divalent ions or } \\
\text { salts present in solution (as they affect the } \\
\text { hydrodynamic volume of the polymer). This method is } \\
\text { also quite slow, typically only three to five samples per } \\
\text { hour (37). }\end{array}$ \\
\hline
\end{tabular}




\section{$2.5 \quad U V$-Vis spectroscopy as a polymer dose monitoring method}

Ultraviolet-visible light (UV-Vis) spectroscopy is a simple, easy to use technology that has potential application as a method for the detection and optimization of residual polymer in drinking water treatment. Gibbons and Örmeci have shown this method to be successful in the determination of polymer concentrations in both water and sludge centrate samples (39). Al Momani and Örmeci also studied the use of UV-Vis spectroscopy for the measurement of residual polyacrylamide polymers in drinking water using an in-line UVvis spectrophotometer. This study showed that the absorbance reading of a sample dosed with a predetermined polymer concentration at $191.5 \mathrm{~nm}$ was able to form a strong linear correlation with the concentration of polyacrylamide present in the given sample $\left(\mathrm{R}^{2}>0.97\right)$. The study was also able to determine the detection limits of the UV-Vis spectrophotometer to be $0.05 \mathrm{mg} / \mathrm{L}$ of polymer in distilled water, and between $0.07 \mathrm{mg} / \mathrm{L}$ to $1.35 \mathrm{mg} / \mathrm{L}$ for water and wastewater samples depending on the polymer type and water quality (22).

\subsubsection{Overview of $U V$-Vis spectroscopy}

All optical spectroscopy technologies function based on the Bohr-Einstein frequency relationship, shown below in Equation 1. This relationship correlates the molecular energy states of a given molecule $\left(E_{i}\right)$ to the frequency of the electromagnetic radiation $(v)$ using a proportionality constant known as Planck's constant $(h)$.

$$
\Delta E=E_{2}-E_{1}=h v \quad \text { Equation 1: Bohr-Einstein frequency relationship }
$$


In spectroscopy applications, the frequency can be related to the velocity of light in a vacuum $(c)$ divided its wavelength $(\lambda)$, thus replacing the frequency value in Equation 1 to give Equation 2, where ( $\tilde{v})$ is known as the wavenumber (40).

$$
\Delta E=E_{2}-E_{1}=\frac{h c}{\lambda}=h c \tilde{v} \quad \text { Equation 2: Bohr-Einstein frequency relationship }
$$

In the ultraviolet to visible light range, the wavelength ranges from $\sim 190 \mathrm{~nm}$ to $800 \mathrm{~nm}$, depending on the spectrophotometer. A complete schematic of the electromagnetic spectrum wavelengths for light photons, depicted below in Figure 7.

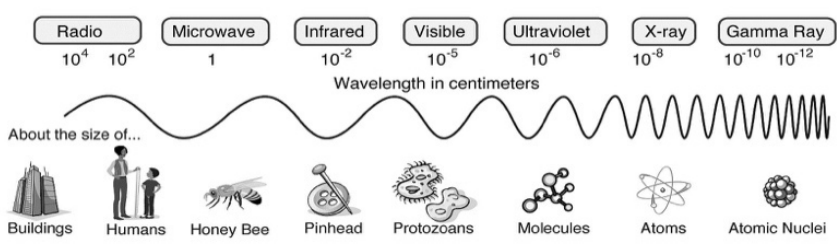

Figure 7: Electromagnetic spectrum schematic (National Geographic, 2018)

The basic concept of UV-Vis spectroscopy is based on the interaction of light with a given medium, where the radiant light can be either absorbed, transmitted, scattered, reflected or excite fluorescence. UV-Vis spectrophotometry is concerned with the transmission and absorption of light radiation through a sample medium and is typically used as a quantitative analysis method for determining the amount of a compound in a solution. A light source that produces visible light, typically a tungsten bulb, and a light source that produces ultraviolet light, typically a deuterium bulb, are used to transmit an incident 
number of light photons, or incident intensity $\left(I_{o}\right)$, over their respective wavelength ranges through a monochromator to a sample cuvette of a specific width or path length $(l)$. This is accomplished under conditions that aim to keep any reflection, scatter, and fluorescence effects to a minimum. A visual representation of the internal workings of a spectrophotometer is depicted below in Figure 8. From here, the number of light photons transmitted, or intensity of the light beam transmitted through the sample $(I)$, is then analyzed by a detector to produce a resulting absorbance value (41).

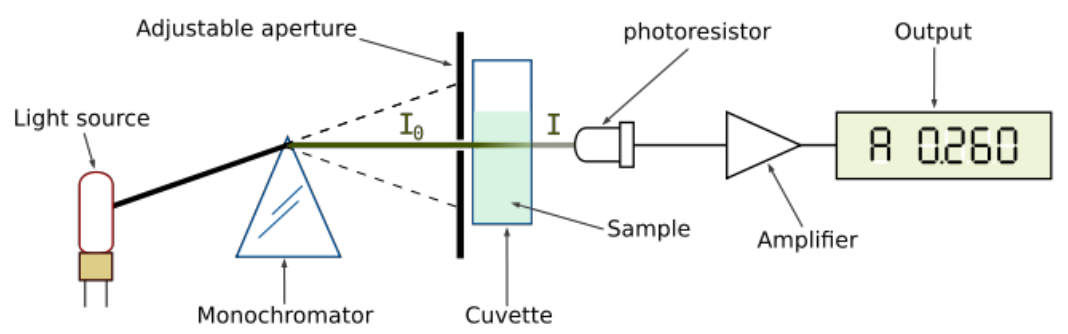

Figure 8: UV-Vis spectrophotometer internal workings schematic (Sigma Aldrich, USA)

The ratio of incident intensity and transmitted intensity gives an indication of what fraction of light entering the sample was able to pass through the sample. This value is referred to as transmittance $(T)$ as described in Equation 3.

$$
T=\frac{I_{o}}{I}
$$

Equation 3: Transmittance

Using transmittance, we can mathematically define absorbance $(A)$ as the negative log of transmittance, as depicted in Equation 4 below. Absorbance is a direct measurement of the amount of light that was absorbed by the sample for a given wavelength. 


$$
A=-\log _{10}\left(\frac{I_{o}}{I}\right)=-\log _{10}(T)
$$

Using these two values we can now define the Beer-Lambert Law, defined as Equation 5, which forms the mathematical-physical basis of spectroscopy in the ultraviolet and visible light regions and is the principle by which spectrophotometry is possible. This law is valid under two main assumptions:

- Assumption 1: The absorbance is directly proportional to the concentration of the sample solution. This is described in the relationship below, where $(A)$ is the measured absorbance and $(C)$ is the sample concertation.

$$
A \propto C
$$

- Assumption 2: The absorbance is also directly proportional to the path length ( $l$ ) of the cuvette holding the sample. This is depicted in the relationship below.

$$
A \propto l
$$

Using these two assumptions combined with Equation 4, as well as the molar absorptivity (ع) as a linear relation coefficient, we can derive the equation for the Beer-Lambert law, relating the sample absorbance to the concentration of the sample.

$$
A=-\log _{10}\left(\frac{I_{o}}{I}\right)=\varepsilon l C
$$

Equation 5: Beer-Lambert Law 


\subsection{Determination of optimum polymer dose}

The effectiveness of a polymer for coagulation and flocculation processes is dependent on the type of polymer used, the dosage, the $\mathrm{pH}$ of the solution, as well as the concentration and nature of the organic compounds present in the water matrix (19). Focusing on parameters that can be controlled, maintaining the polymer dose at an optimal level during treatment is a method of maintaining effectiveness that would be beneficial for operation, however is not as straight forward to implement as simply selecting the correct polymer. The organic compounds present in the influent are constantly subject to change, which in turn reflects on the optimum polymer dose required for treatment.

The standard method for the optimization of coagulant and/or flocculant dose in water, wastewater and sludge applications in the jar test, and has been for over half a century (42) (43). Jar testing is a pilot-scale simulation of a coagulation/flocculation process that is performed in order to determine the most effective dosage of a given coagulant or flocculant for a particular water treatment operation (44). This method involves dosing the polymer at varying concentrations to identical water samples and observing their settling time simultaneously. The optimum dose typically corresponds to the fastest settling time, the heaviest floc, as well as the lowest supernatant turbidity reading (44). Capillary suction time (CST) tests, settling tests and filtration tests are also frequented for optimizing polymer dose in wastewater and sludge applications, however these methods are typically not feasible for the low polymer doses in drinking water applications (45) (46). Although the jar (settling) test is an effective method for estimating optimum polymer dose in drinking water, it is not practical to execute these tests in-line continuously during the 
treatment process, as they require laboratory access and time to complete. If this method is applied in operation it can be timely to execute and is often only done seasonally, leading to inaccurate optimum dosages based on the changing water matrix in between the performance of the jar tests.

$\mathrm{UV}$-vis spectroscopy is a potential method for the in-line optimization of polymer dosage for drinking water applications. Al Momani and Örmeci were able to use this method to determine the optimal polymer dose range for sludge dewatering in-line by establishing a relationship between the optimum polymer dose and the UV-vis absorbance readings of the sludge filtrate at $191.5 \mathrm{~nm}$, which correspond to the concentration of residual polymer in the filtrate (23). The optimum dose was first determined using standard methods, namely capillary suction time (CST) tests and filtration tests, which was then used to determine a relationship between the filtrate absorbance at $191.5 \mathrm{~nm}$ and the optimum dose. This method was successful in demonstrating this relationship, exhibiting a decrease in filtrate absorbance in the under-dose range as polymer dose is increased, and an increase in filtrate absorbance in the over-dose range as polymer dose was increased. The optimal dosage range corresponded to the minimum filtrate absorbance reading at $191.5 \mathrm{~nm}$ (the curve forming a ' $U$ ' or ' $V$ ' shape), indicating that the real-time optimization of polymer addition using UV-vis spectroscopy has the potential to be applied at wastewater treatment plants. This is an advantage of UV-vis spectroscopy over traditional jar testing because where turbidity would remain constant beyond optimum dose; absorbance would increase, making the optimum dose more detectable. Salam, Örmeci and Simms also studied this method for the determination of the optimum polymer dose in the dewatering of oil sands 
tailings, and a similar relationship was acquired, the sample absorbance at 190nm was found to correlate linearly with the residual polymer concentration in the tailings water (47). This same relationship may also have the potential to be applied to drinking water applications by firstly determining the optimal polymer dose using the jar test method and measuring the supernatant turbidity, and then correlating these results to the minimum supernatant absorbance at 190nm using UV-vis spectroscopy.

\subsection{Actiflo ${ }^{\circledR}$ treatment process}

To test the objectives of this study, a drinking water treatment process that utilizes polyacrylamide polymer during the coagulation and flocculation stage was chosen for

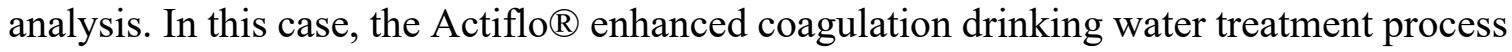
utilized by the Casselman municipal drinking water treatment facility in Casselman, ON was mimicked for laboratory purposes. This facility treats their source water using the Actiflo ${ }^{\circledR}$ clarifying process followed by the Dunsenflo ${ }^{\circledR}$ filtration unit, both of which are manufactured by Veolia Water Technologies, Inc. At the Casselman facility, potassium permanganate $(\mathrm{KMnO} 4)$ is added to the raw water initially in order to oxidize any iron and/or manganese that may be present. Afterwards, a polyaluminum sulphate coagulant manufactured by Kemira Inc., known PASS-10, is dosed into the inlet stream before the water enters the clarifier. These constituents are mixed in a coagulation mixing tank to destabilize colloidal materials. Following this mixing, the water is sent to an injection tank where an anionic polyacrylamide polymer manufactured by BASF Canada Inc. known as Magnafloc LT27AG is added, along with microsand to assist in weighing down the flocs that will be formed and facilitate settling later on. These constituents are mixed gently to 
accelerate the bridging mechanism between pin flocs, suspended solids and microsand. After a maturation period, the water is sent to the decantation tank where the sand and now formed flocs are settled out. This effluent is then sent to the Dunsenflo ${ }^{\circledR}$ filtration unit, where any remaining suspended particulates are filtered through a combination of sand and anthracite (48). A complete schematic of the Actiflo ${ }^{\circledR}$ clarifying process and the Dunsenflo ${ }^{\circledR}$ filtration unit is outlined in Figure 9.

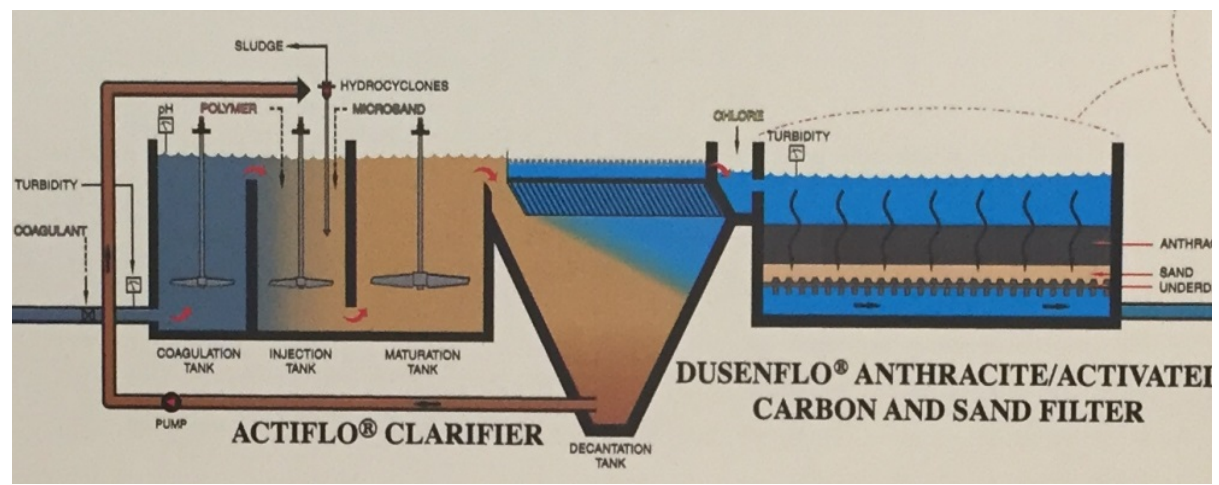

Figure 9: Actiflo clarification process and Dunsenflo filtration unit schematic (Casselman Treatment Facility, 2016) 


\section{CHAPTER 3: MATERIALS AND METHODS}

\subsection{Polymers}

In the first, second and third phases of experiments, an anionic polyacrylamide polymer known as Magnafloc LT27AG manufactured by BASF Inc. was used due to the fact that it is the primary polymer used in the Actiflo ${ }^{\circledR}$ treatment process carried out at the Casselman drinking water treatment facility, located in Casselman, ON. In the final phase of experiments, a cationic polyacrylamide polymer known as Hydrex 3661 manufactured by Veolia Inc. was also tested in order to ensure the determination of optimum polymer dose using the proposed method was viable for both a cationic and anionic polymer. Both of these polymers are typical for potable water treatment use (22). A summary of relevant characteristics of each polymer are listed in Table 2.

Table 2: Polymer Characteristics

\begin{tabular}{lccccc}
\hline \multicolumn{1}{c}{ Polymer } & Type & Charge & Product Use & Appearance & $\begin{array}{c}\text { Density } \\
\left(\mathbf{k g} / \mathbf{m}^{\mathbf{3}}\right)\end{array}$ \\
\hline Magnafloc LT27AG & Polyacrylamide & Anionic & Potable water treatment & Crystalline Powder & 750 \\
\hline Hydrex 3661 & Polyacrylamide & Cationic & Potable water treatment & Crystalline Powder & $600-900$ \\
\hline
\end{tabular}

\subsection{Preparation of polymer stock solution}

Stock solutions of both the anionic and cationic polymers were prepared at $400 \mathrm{mg} / \mathrm{L}$ concentrations for sample spiking purposes. Firstly, $0.20 \mathrm{~g}$ of crystalline polymer weighed using a lab scale (Sartorius BP2215 model). Using a jar testing apparatus (Phipps and Bird, USA), the granulated polymer was added to $500 \mathrm{~mL}$ of deionized water and mixed at 220 
RPM for 5 minutes. Afterwards, the stirring speed was reduced to 125 RPM for 55 minutes. If any large flocs were visible in the solution, a hand mixer (Braun, Germany) was used post-mixing for 10 -second pulse intervals as needed to produce a well-mixed, homogenous solution. The solution was then left to mature for a minimum of 1 hour prior to experimental use. This procedure produced a well-dissolved polymer solution for both polymers. Fresh polymer stock solutions were prepared weekly over the course of the experiment.

\subsection{Water samples}

Source waters vary in physical and chemical characteristics; therefore, a variation of water matrices was used throughout the duration of the experiment. During the first phase of the experiment, deionized water was used as a control in order to compare the effect of path length on residual polymer detection, as well as the difference in sensitivity and detection limits between desktop and in-line spectrophotometers. The second and third phases of the experiment aimed to determine the effects of filtration and dilution, as well as the Actiflo ${ }^{\circledR}$ treatment process chemical additives the sensitivity of UV-vis spectroscopy for residual polymer detection. This was achieved using raw water collected from the Rideau River in Ottawa, ON, and deionized water as a controlled comparison. The Rideau River water used for the second and third phases of the experiment had an average turbidity of 17NTU. The fourth phase of the experiment aimed to use UV-vis spectroscopy to determine the optimum polymer dose for a given water sample. For this purpose, raw water from the Rideau River in Ottawa, ON was collected at both high turbidity (4000-9000 NTU) and low turbidity (200-600 NTU). 


\subsection{Sample preparation}

Samples were prepared for this study in four main experimental phases, as depicted below in Figure 10. Each of these phases is further explained in the following subsections.
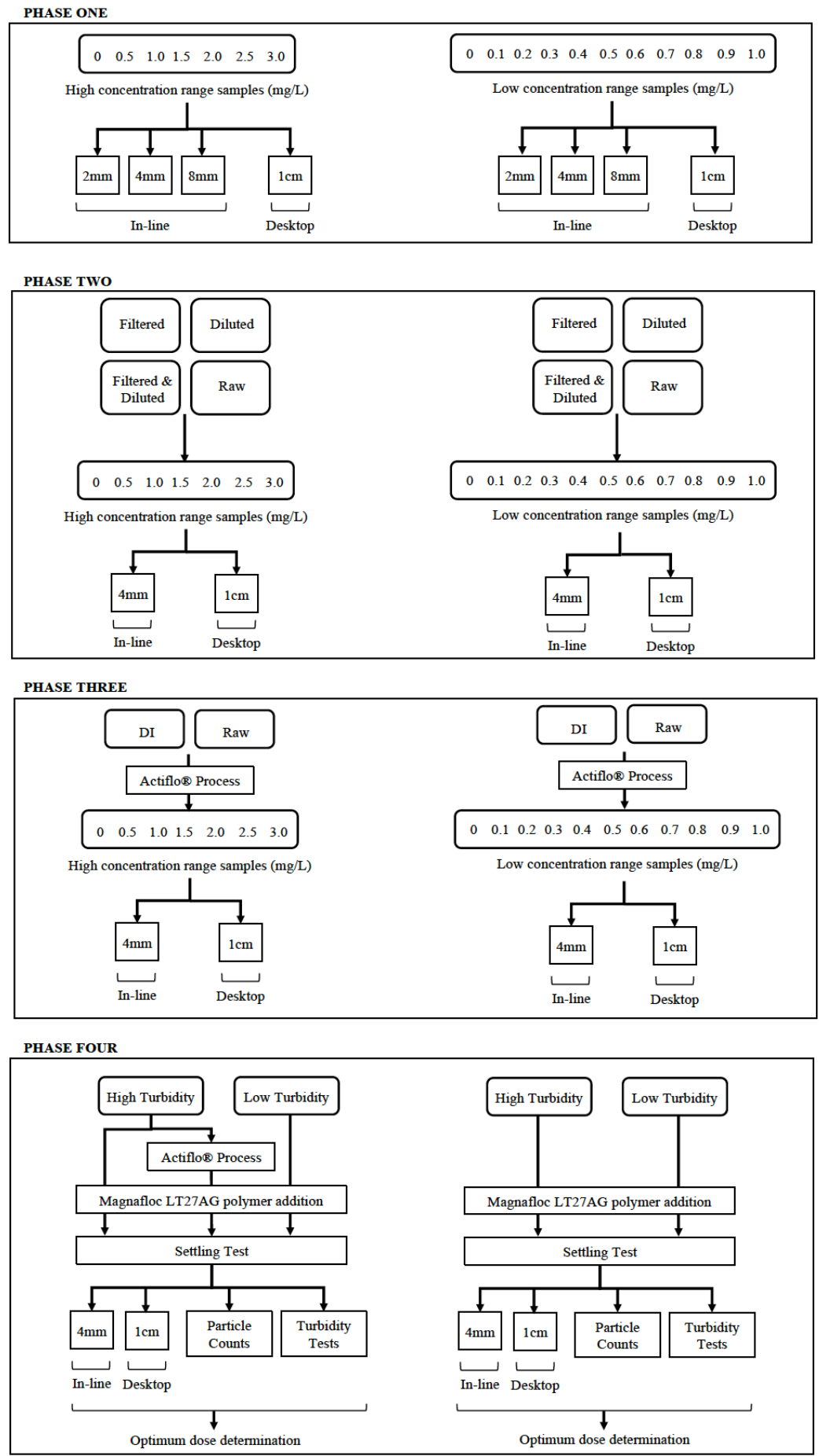

Figure 10: Experimental phase outline schematic 


\subsubsection{Phase 1}

Samples for the first phase of the experiment were prepared by spiking deionized water with the prepared Magnafloc LT24AG polymer (400mg/L stock solution) at both a high concentration range $(0-3 \mathrm{mg} / \mathrm{L})$ and a low concentration range $(0-1 \mathrm{mg} / \mathrm{L})$ to prepare $400 \mathrm{~mL}$ samples of each polymer dose. For the high concentration range samples $(0-3$ $\mathrm{mg} / \mathrm{L}$ ), polymer stock solution was pipetted into the prepared sample beakers in increments of $0,0.5,1.0,1.5,2.0,2.5$ and $3.0 \mathrm{~mL}$. For the low concentration range samples $(0-1 \mathrm{mg} / \mathrm{L})$, polymer solution was pipetted into the prepared sample beakers in increments of $0,0.1$, $0.2,0.3,0.4,0.5,0.6,0.7,0.8,0.9$ and $1.0 \mathrm{~mL}$. After polymer addition, the samples were mixed for 1 minute using a jar test apparatus (Phipps and Bird, USA) at 125RPM, prior to being used for absorbance measurements.

\subsubsection{Phase 2}

Samples for the second phase of the experiment were prepared using water collected from the Rideau River on Carleton University Campus in Ottawa, ON. Samples of Rideau River water with turbidity measurements of $6.8 \mathrm{NTU}$ and 3.2 NTU were collected; half of each was set aside for filtration. These sample sets were prepared using raw Rideau River water, filtered Rideau River water, diluted Rideau River water, as well Rideau River water that was both filtered and diluted. Each sample was prepared by spiking the water with the prepared Magnafloc LT24AG polymer (400mg/L stock solution) at both a high concentration range $(0-3 \mathrm{mg} / \mathrm{L})$ and a low concentration range $(0-1 \mathrm{mg} / \mathrm{L})$ to prepare $400 \mathrm{~mL}$ samples of each polymer dose. For the high concentration range samples (0- 
$3 \mathrm{mg} / \mathrm{L}$ ), polymer solution was pipetted into the prepared sample beakers in increments of $0,0.5,1.0,1.5,2.0,2.5$ and $3.0 \mathrm{~mL}$. For the low concentration range samples $(0-1 \mathrm{mg} / \mathrm{L})$, polymer solution was pipetted into the prepared sample beakers in increments of $0,0.1$, $0.2,0.3,0.4,0.5,0.6,0.7,0.8,0.9$ and $1.0 \mathrm{~mL}$.

\subsubsection{Dilution and filtration of samples}

The second phase of the experiment analyzed the effects of suspended particulates through dilution of the samples. The raw water samples were diluted using deionized water after polymer addition and before absorbance measurements. The following dilution procedure was used. Firstly, 400mL samples of Rideau River water were spiked with polymer at both a high concentration range $(0-3 \mathrm{mg} / \mathrm{L})$ and a low concentration range $(0-1 \mathrm{mg} / \mathrm{L})$ in increments of $0.5 \mathrm{mg} / \mathrm{L}$ and $0.1 \mathrm{mg} / \mathrm{L}$, respectively. The sample and polymer mixtures were then mixed for 1 minute using a jar test apparatus (Phipps and Bird, USA) at 125RPM, and an absorbance spectrum of each sample was measured. Afterwards, each sample was diluted using deionized water at a ratio of 1:1, and their absorbance spectra were measured a second time.

The second phase of the experiment also explored the effects of suspended particulates through filtration of the samples. The raw water samples were filtered using a Whatman $0.45 \mu \mathrm{m}$ filter paper (Sigma Aldrich, USA) prior to polymer addition and absorbance measurements. In the instances where filtration and dilution were combined on the sample, the raw water samples were filtered first and then the dilution procedure was carried out. 
Table 3 below outlines the filtration and dilution scheme of the samples prepared during phase 2 .

Table 3: Phase 2 sample summary

\begin{tabular}{|c|c|c|c|}
\hline $\begin{array}{c}\text { Magnafloc LT27AG Polymer } \\
\text { Dose Range }\end{array}$ & $\begin{array}{c}\text { Raw Rideau River Water } \\
\text { Turbidity (NTU) }\end{array}$ & Filtration & Dilution \\
\hline \multirow{3}{*}{ High Range (0-3 mg/L) } & \multirow{3}{*}{6.8} & $\mathrm{x}$ & \\
\hline & & & $\mathrm{x}$ \\
\hline & & $\mathrm{x}$ & $\mathrm{x}$ \\
\hline \multirow{3}{*}{ Low Range (0-1 mg/L) } & \multirow{3}{*}{3.2} & $\mathrm{x}$ & \\
\hline & & & $\mathrm{x}$ \\
\hline & & $\mathrm{x}$ & $\mathrm{x}$ \\
\hline
\end{tabular}

\subsubsection{Phase 3}

The third phase of the experiment required the imitation of the Actiflo ${ }^{\circledR}$ enhanced coagulation treatment process utilized by the Casselman drinking water treatment facility in Casselman, ON. These samples were prepared using water collected from the Rideau River on Carleton University Campus in Ottawa, ON with a turbidity of 32.0 NTU, as well as deionized water as a controlled comparison. These samples were prepared by mimicking the full-scale enhanced coagulation process at a laboratory scale, adding each chemical in the same succession and dosage as would be done during full-scale operation. Each sample maintained a constant concentration of the Actiflo ${ }^{\circledR}$ treatment process chemicals, varying only the concentration of polymer as well as the concentration of sodium hydroxide necessary to achieve a neutral $\mathrm{pH}$. 
Each $400 \mathrm{~mL}$ sample was prepared by firstly adding potassium permanganate acquired from the Casselman treatment facility to the raw sample at a dose of $1.0 \mathrm{mg} / \mathrm{L}$ in order to oxidize any iron or manganese present in the water. This is the typical dose for drinking water treatment purposes as reported by the United States Environmental Protection Agency (US EPA). The potassium permanganate is supplied in powder form, and therefore $0.0004 \mathrm{~g}$ had to be weighed using a weighing scale (Sartorius BP2215 model) for each sample. Following this, a polyaluminium silicate sulphate (PASS-10) coagulant manufactured by Kemira Inc. (Helsinki, Finland) acquired from the Casselman treatment facility was added to each sample at a dose of $50 \mathrm{mg} / \mathrm{L}$, a typical dosage as reported by the Casselman treatment facility. The PASS-10 coagulant is supplied as a solution and was therefore added to each sample by pipetting $40.8 \mu \mathrm{L}$ to each sample. The Magnafloc LT27AG polymer $(400 \mathrm{mg} / \mathrm{L}$ stock solution) was then dosed to the samples at both a high concentration range $(0-3.0 \mathrm{mg} / \mathrm{L})$ and a low concentration range $(0-1.0 \mathrm{mg} / \mathrm{L})$, along with $0.80 \mathrm{~g}$ of microsand to aid in settling by facilitating the formation of larger, heavier flocs. Table 4 summarizes all chemicals used for the Actiflo ${ }^{\circledR}$ treatment process.

Table 4: Actiflo treatment process additives summary

Treatment Additive

Magnafloc LT27AG polymer

PASS-10 Coagulant

Microsand

Potassium Permanganate

Sodium Hydroxide

\section{Dosage concentration}

$400 \mathrm{mg} / \mathrm{L}$ stock in $0-1 \mathrm{mg} / \mathrm{L}$ and $0-3.0 \mathrm{mg} / \mathrm{L}$ concentration ranges $50 \mathrm{mg} / \mathrm{L}$

$2 \mathrm{~kg} / \mathrm{m}^{3}$

$1.0 \mathrm{mg} / \mathrm{L}$

As needed to reach $\mathrm{pH}$ of 7.0 
For the high concentration range samples $(0-3 \mathrm{mg} / \mathrm{L})$, polymer solution was pipetted into the prepared sample beakers in increments of $0,0.5,1.0,1.5,2.0,2.5$ and $3.0 \mathrm{~mL}$. For the low concentration range samples $(0-1 \mathrm{mg} / \mathrm{L})$, polymer solution was pipetted into the prepared sample beakers in increments of $0,0.1,0.2,0.3,0.4,0.5,0.6,0.7,0.8,0.9$ and 1.0 $\mathrm{mL}$. Following the addition of the chemicals and polymer, the samples were mixed for two minutes using a jar test apparatus (Phipps and Bird, USA) at 125RPM. Post-mixing, a pH meter (ThermoFisher Scientific, Waltham, US) was used to measure the $\mathrm{pH}$ of each sample. A $1 \mathrm{~N}$ solution of sodium hydroxide $(\mathrm{NaOH})$ was then added in increments of $15 \mu \mathrm{L}$ as needed to neutralize the $\mathrm{pH}$ in accordance with the process carried out at the Casselman treatment facility. On average, $100 \mu \mathrm{L}$ of $\mathrm{NaOH}$ solution was added to each sample in order to achieve a $\mathrm{pH}$ of seven. The samples were then left to settle for thirty minutes, again in accordance with the sedimentation process carried out at the Casselman treatment facility. The supernatant of each settled sample was then used for absorbance measurements.

\subsubsection{Phase 4}

Samples for the fourth phase of the experiment were prepared using Rideau River water collected from the Carleton University Campus in Ottawa, ON. The water was to be analyzed at both a very high turbidity, and a lower turbidity. For this purpose, a clay-like soil was added until the desired turbidity was reached. The high turbidity samples ranged from $4000-9000$ NTU, and the low turbidity samples ranged from $200-600$ NTU.

For both the high and low turbidity water samples, a series of settling test trials were performed to determine the optimum polymer dose range for both the Magnafloc LT27AG 
as well as the Hydrex 3661 polymers. These trials were done by firstly measuring $200 \mathrm{~mL}$ of raw Rideau River water and spiking with polymer solution in increments of $1.0 \mathrm{mg} / \mathrm{L}$. After spiking, the samples were mixed for 1 minute using a jar test apparatus (Phipps and Bird, USA) at 125RPM. The mixed samples were then left to settle, and the apparent turbidity of each sample was observed while the settling time was being recorded. The range of dosages that reached an apparent clarity in the least amount of settling time were selected as the optimum polymer dose range. Once this range was determined, a series of polymer doses were chosen to represent this optimum dose range, an over dose range, and an under-dose range. Combining all of these ranges, a sample set was created for both the high and low turbidity Rideau River water samples, for both the Magnafloc LT27AG and Hydrex 3661 polymers.

\subsubsection{Settling tests}

In order to determine the precise optimum dose of each polymer at each turbidity, a proper settling test was carried out based on the pre-determined ranges from the earlier performed trials. The procedure for the settling tests was as follows. Firstly, $400 \mathrm{~mL}$ samples of each polymer dosage determined during the trial period were prepared by spiking the raw water with the prepared polymer solution to reach the desired sample concentration. This was done using both the Magnafloc LT27AG polymer (400mg/L stock solution) as well as the Hydrex 3661 polymer (400mg/L stock solution). After spiking, the samples were mixed simultaneously for 2 minutes at 125RPM using a jar test apparatus (Phipps and Bird, USA). Immediately after mixing, $50 \mathrm{~mL}$ of the combined solution was pipetted from the sample beakers into clear, plastic $50 \mathrm{~mL}$ flasks. The flasks were then mixed once more for 10 
seconds before settling. The settling time was recorded as all of the samples settled simultaneously. During the settling process, the samples were observed until the first of the set reached maximum clarity and the settling ended. At this point the settling time was recorded and the interfaces of all samples were measured in the $50 \mathrm{~mL}$ flasks. The supernatant was then extracted from each sample of varying polymer dose using a pipette and set aside for turbidity measurements, absorbance measurements as well as particle counts.

This settling process was repeated using the Actiflo ${ }^{\circledR}$ treatment process, following the same procedure as that of the phase 3 sample preparations. The Actiflo ${ }^{\circledR}$ treatment chemicals were added to the high turbidity Rideau River water samples prior to performing the settling tests. Again, prior to performing the proper settling test procedure, a series of settling test trials were performed to determine the optimum polymer dose range for the Magnafloc LT27AG polymer in the same method as for that without the addition of the Actiflo ${ }^{\circledR}$ chemicals. The Actiflo ${ }^{\circledR}$ treatment process was only tested for the MagnaflocLT27AG polymer, as it is the polymer used for the process at the Casselman water treatment facility. The proper settling test procedure was then followed, and the supernatant was again extracted from each sample of varying polymer dose using a pipette and set aside for turbidity measurements, absorbance measurements as well as particle counts. 


\subsection{Analytical Methods}

\subsubsection{Turbidity measurements}

Phase 4 of the experiment utilized turbidity measurements of the settled sample supernatants to compare polymer effectiveness and determine the optimum dose. The procedure for measuring the turbidity is as follows. Supernatant collected from the settling test samples in phase 4 where transferred into clean, individual HACH turbidity test tubes. A HACH (HACH Co., USA) turbidimeter was calibrated according to factory instructions prior to each set of tests. The samples were then inverted in the HACH tubes 10 times immediately before inserting the tubes into the turbidimeter. The meter was then read and documented when the value displayed remained at a value $+/-5$ NTU. This entire process was carried out three times per sample, and the average of the three values was taken as the sample's overall turbidity value.

\subsubsection{UV-Vis Spectroscopy Absorbance measurements}

Absorbance measurements were taken for all samples in phases 1 through 4 using both an in-line UV-Vis spectrophotometer, as well as a desktop UV-vis spectrophotometer. The samples were divided after preparation to ensure an equal comparison between absorbance values on each spectrophotometer; $10 \mathrm{~mL}$ of each sample was reserved for use in the desktop unit and the remaining 390mL was reserved for use in the in-line unit. Absorbance spectrums were read for each sample from $189.5 \mathrm{~nm}-800 \mathrm{~nm}$ for the in-line unit, and 190nm - 800nm for the desktop unit based on the spectral capabilities of each spectrophotometer. It has been determined that the polymers chosen for analysis in this experiment show a 
maximum absorbance at $190 \mathrm{~nm}$, and these values were therefore taken as the overall result value for each sample concentration (Gibbons \& Örmeci, 2013). The in-line UV-Vis spectrophotometer (Real Spectrum Platinum Series) is manufactured by Real Tech Inc. (Ontario, Canada) and allows for a real-time analysis of the sample's absorbance across the entire spectrum of ultraviolet and/or visible light $(189.5 \mathrm{~nm}-800 \mathrm{~nm})$. Figure 11 below illustrates the inner workings of the in-line spectrophotometer.

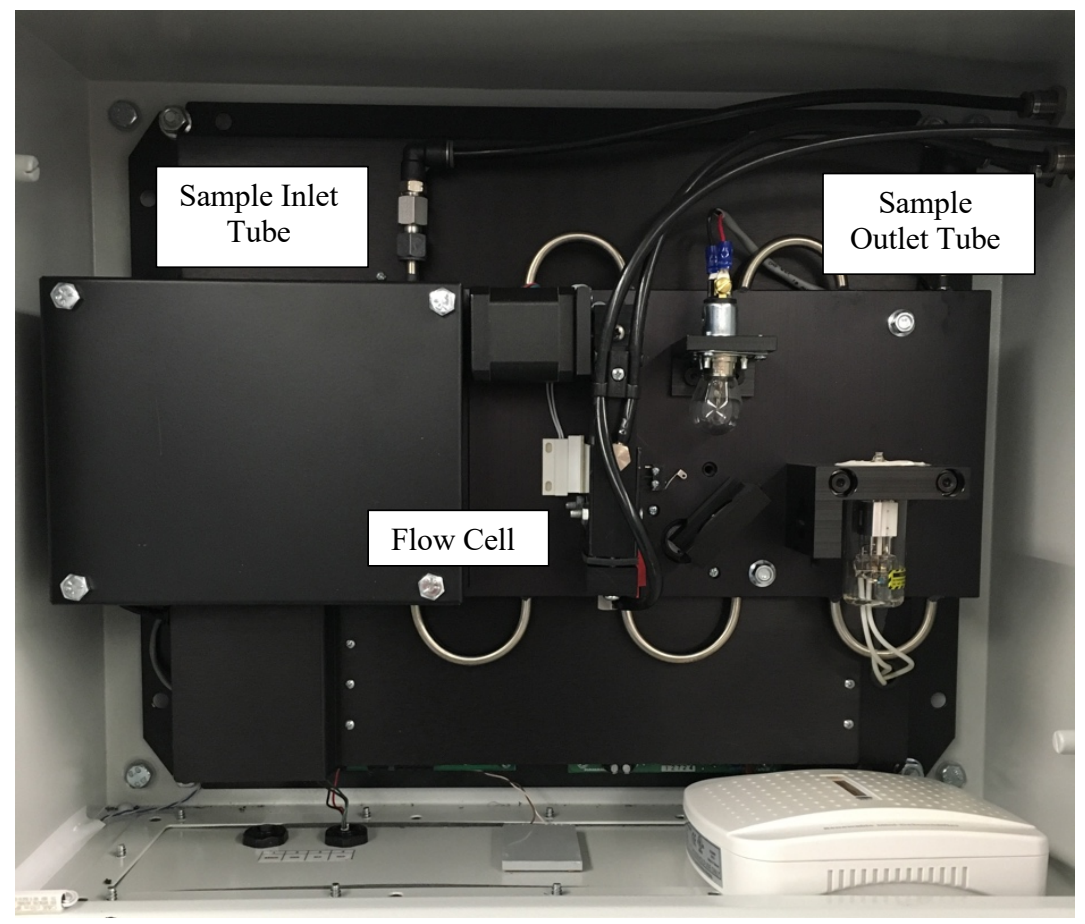

Figure 11: In-line spectrophotometer (Real Tech Inc.)

The experimental setup consisted of the in-line spectrophotometer mounted onto a wall in the Civil and Environmental Engineering lab located at Carleton University in Ottawa, ON. The spectrophotometer itself includes a tungsten lamp as the visible light source, a deuterium lamp as the ultraviolet light source, a PL sensor for interpreting absorbance measurements (Real Tech Inc., ON), a dehumidifier (EvaDry, Tampa, US), a pump and 
tubing for inlet/outlet sample flow, and a quartz flow cell (Real Tech Inc., ON). The flow cells used were in path lengths of $2 \mathrm{~mm}, 4 \mathrm{~mm}$ and $8 \mathrm{~mm}$, and were also supplied by Real Tech Inc. Each flow cell was able to detect polymer at various levels of precision in deionized water, the $2 \mathrm{~mm}$ path length had an average standard deviation of \pm 0.0027 A.U., the $4 \mathrm{~mm}$ path length had an average standard deviation of \pm 0.0007 A.U., and the $8 \mathrm{~mm}$ path length had an average standard deviation of \pm 0.0012 A.U. between absorbance measurements for a given polymer concentration. The desktop UV-vis spectrophotometer (Cary-100 Bio series) is manufactured by Varian Inc./Agilent Technologies (USA). The desktop spectrophotometer is a double beam unit equipped with a tungsten halogen visible light source with a quartz window, a deuterium arc ultraviolet light source, and is centrally controlled by an external computer. The experimental set up consists of the unit itself and the external computer source, and a 10mm Hellma quartz cuvette (Hellma Canada Ltd., Canada) which was loaded into the sample holder located inside the unit.

The procedure for running a sample through the in-line spectrophotometer is as follows. Firstly, the unit was equipped with a flow cell of desired path length depending on the nature of the experiment. During phase one, the flow cell was changed between $2 \mathrm{~mm}, 4 \mathrm{~mm}$ and $10 \mathrm{~mm}$ for both the high polymer concentration range $(0-3 \mathrm{mg} / \mathrm{L})$ and low polymer concentration range $(0-1 \mathrm{mg} / \mathrm{L})$ sample sets. This was also altered on the systems internal software by changing the 'path length' field to the corresponding value for each flow cell. Once the desired flow cell was installed, the unit was turned on and allotted a minimum of 30 minutes to run through distilled water in accordance with the equipment's start-up procedure. The wavelength range was set from $189 \mathrm{~nm}-350 \mathrm{~nm}$ on the systems internal 
software. Afterwards, the unit was zeroed with deionized water before beginning any sample analysis. This was done by allowing the deionized water to run through for a minimum of 10 minutes prior to executing the programs 'zero' function. The absorbance values were then observed for 2 consecutive readings prior to beginning analysis to ensure the spectrophotometer was properly zeroed. Samples prepared earlier were then mixed with a stirring stick in a beaker for 30 seconds prior to being loaded onto the inlet tubing of the spectrophotometer. The sample was then allowed to run through the machine for 3 minutes, with the outlet being diverted into a waste beaker. After 3 minutes, the deionized water had been flushed out and the sample occupied all tubing volume. At this point, the outlet was diverted back into the inlet sample beaker to allow for a recycle of sample through the machine. This was done to accommodate the sample volume capacity necessary for the unit to analyze the sample a minimum of 3 consecutive analysis cycles. Each sample cycle requires 1 minute and 55 seconds. The average of these three absorbance readings is reported as the absorbance for each respective sample in the results. After 3 analysis cycles, the sample was removed from the inlet and replaced with deionized water for one full cycle to flush out any sample from the units tubing. After this flushing cycle, the next sample was loaded onto the inlet and the procedure was repeated until all samples in the set had been analyzed. Samples were analyzed in order from lowest to highest polymer concentration. After every sample set was complete, the unit was given another 10 minutes to run through deionized water and was re-zeroed prior to beginning to analyze a new sample set. 
The procedure for running a sample through the desktop UV-Vis spectrophotometer is as follows. Firstly, the unit was powered on for a minimum of 1 hour prior to analysis in accordance with the machines start-up procedure in order to give the lamps sufficient time to warm up. The software was set to 'double-beam' setting and the wavelength range was set from $190 \mathrm{~nm}-800 \mathrm{~nm}$. Afterwards, the clean quartz cuvette was filled with $4 \mathrm{~mL}$ of deionized water and loaded into the unit. The 'zero' function was initiated through the system software located on the external computer source. Once zeroed, the cuvette was removed, and any residual water present on the cuvette was dried by means of a compressed air canister to eliminate the possibility of any sample dilution. Samples prepared earlier were then mixed with a stirring stick in a beaker for 30 seconds prior to being poured into the cuvette for analysis. It was ensured that the sample fill the cuvette at minimum $3 / 4$ of the way full, in accordance with the spectrophotometers outlined operating procedure. The sample cuvette was then wiped using a Kim wipe to ensure no dust was present on the quartz walls and was then loaded into the machine where an absorbance spectrum was produced. This measurement was repeated twice per sample, and the reported absorbance measurements are the average of these replicates. After each sample, the cuvette was rinsed with deionized water and dried using the air canister before loading the next sample. This procedure was repeated until all samples within a given sample set had been analyzed, after which point the unit was re-zeroed before beginning a new sample set. Samples were analyzed in order from lowest to highest polymer concentration. 


\subsubsection{Particle counts}

Particle counts of samples produced in phase 4 were carried out using a Dynamic Particle Analyzer (BP-4100-HEV model) manufactured by BrightWell Technologies INC (Ottawa, ON). This unit is capable of analyzing both sub-visible and visible particles. Micro-Flow Imaging $^{\mathrm{TM}}$ (MFI) technology is used in this unit to allow for the rapid measurement of particle size, count, concentration and morphology. The flow cell used (BP-4100-FC-400UN, Brightwell Technologies Inc.) was able to detect particles sized from $100 \mu \mathrm{m}-400 \mu \mathrm{m}$. The experimental set-up consisted of the particle counter itself, a beaker of deionized water that had also been filtered through a vacuum filtration system using Whatman $0.45 \mu \mathrm{m}$ filter paper (Sigma Aldrich, USA) in order to eliminate any possible false particles, a pumping mechanism, an external computer with the system software and the flow cell. The procedure for particle counts was conducted as follows. Firstly, the filtered deionized water was allowed to pump through the system for a minimum of 2 minutes in order to flush the flow cell of any potential particles. A cleaning solution (50\% PCC54 detergent by volume) was then introduced into the inlet and allowed to flush through the system for another 2 minutes, followed by a final flush of deionized water for 1 minute. The systems cleanliness check was then used to ensure the flow cell was clean enough to be used for analysis. The sample in question was then loaded into the inlet tube and a stirring mechanism was initiated at a speed setting of 3 in order to maintain particle suspension. The sample was then run through the system based on a volume of $0.22 \mathrm{~mL} / \mathrm{min}$ in accordance with the software's recommendation for the flow cell used. For each sample set, a cleaning procedure was followed between samples to ensure accurate particle readings. After the sample had finished its analysis, deionized water was allowed to run through the system 
for one minute, followed by another minute of the cleaning solution and then a final minute flush of deionized water. The successive sample was then loaded into the inlet and the procedure was repeated until the entire set had been completed.

\subsection{Statistics}

\subsubsection{Absorbance readings}

All absorbance measurements were taken a minimum of three repetitions per sample. The average of these three readings at each wavelength was then taken as the overall absorbance reading for the production of the absorbance spectra. A minimum of seven data points were recorded at each polymer concentration range. The maximum absorbance values for each concentration recorded at a $190 \mathrm{~nm}$ wavelength from the absorbance spectra were then used to create a linear correlation representing the absorbance-polymer concentration relationship established for each data set. The polymer concentration used for the linear correlation plots refers to the concentration of polymer dosed in the original sample, not the residual polymer concentration post settling. The error bars on these linear correlations represent the standard deviation between the three repetitions of each absorbance measurement for the respective sample concentration. 


\section{CHAPTER 4: RESULTS \& DISCUSION}

\subsection{Effect of path length on polymer detection using UV-vis spectroscopy}

\subsubsection{UV-vis absorbance spectra}

The effect of path length on the detection sensitivity using UV-Vis spectroscopy for the detection of residual polymer was assessed using both a high range of Magnafloc polymer concentration $(0-3 \mathrm{mg} / \mathrm{L})$ as well as a low range $(0-1 \mathrm{mg} / \mathrm{L})$ in deionized water under the above-mentioned increments and conditions. Each concentration range was analyzed using both the desktop spectrophotometer using a $10 \mathrm{~mm}$ path length quartz flow cell, and the inline spectrophotometer using the $2 \mathrm{~mm}, 4 \mathrm{~mm}$ and $8 \mathrm{~mm}$ path length flow cells. The results were then compared to establish which path length produced the most sensitive absorbance results, as well as to compare the sensitivity between both the desktop and in-line spectrophotometers. Absorbance readings were taken from 190nm to $350 \mathrm{~nm}$. Figures 12a and $12 \mathrm{~b}$ below represent the absorbance spectra for the $0-3 \mathrm{mg} / \mathrm{L}$ and $0-1 \mathrm{mg} / \mathrm{L}$ polymer concentration range respectively using the desktop spectrophotometer and a $10 \mathrm{~mm}$ flow cell. 
(a) High Concentration Range

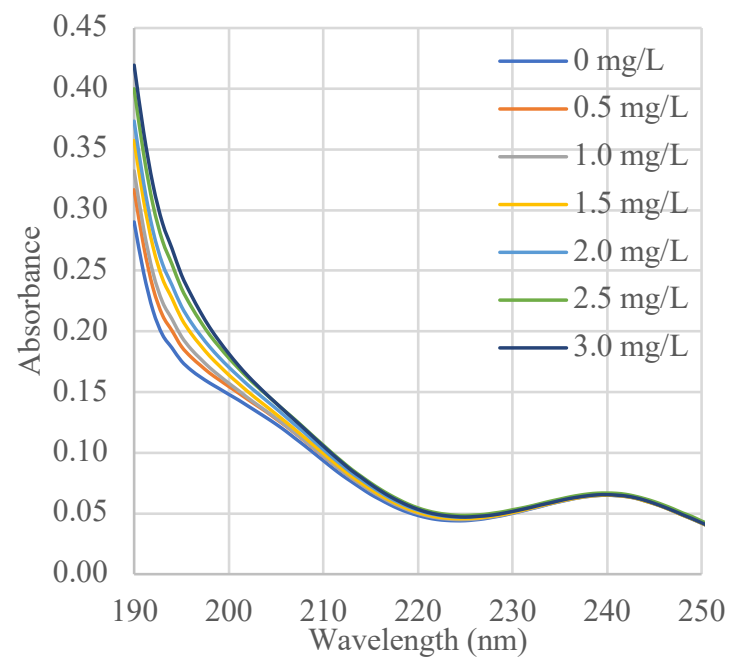

(b) Low Concentration Range

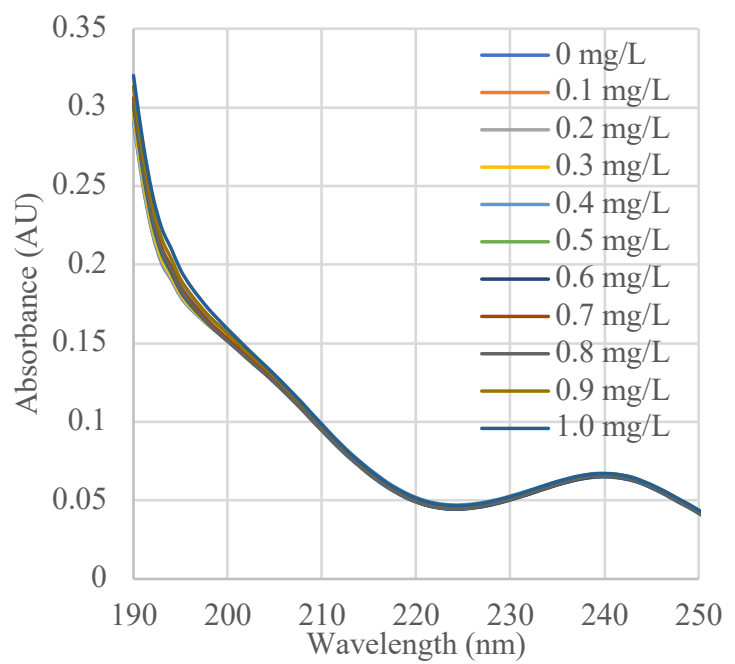

Figure 12: Absorbance spectra of Magnafloc LT27AG polymer in deionized water using a desktop spectrophotometer with a $10 \mathrm{~mm}$ flow cell in the concentration ranges of (a) $0-3 \mathrm{mg} / \mathrm{L}$ and (b) $0-1 \mathrm{mg} / \mathrm{L}$

Figures $13 \mathrm{a}$ and $13 \mathrm{~b}$ represent the absorbance spectra for the $0-3 \mathrm{mg} / \mathrm{L}$ and $0-1 \mathrm{mg} / \mathrm{L}$ polymer concentration using the in-line spectrophotometer and a $2 \mathrm{~mm}$ flow cell. The flow cell was then increased to $4 \mathrm{~mm}$ and the same readings were taken, as represented by Figures $14 \mathrm{a}$ and $14 \mathrm{~b}$. Finally, the flow cell was increased to $8 \mathrm{~mm}$ and the absorbance spectra were reproduced under these conditions, as represented by Figures $15 \mathrm{a}$ and $15 \mathrm{~b}$. Previous studies by Al Momani and Örmeci as well as Gibbons and Örmeci showed no significant absorbance readings beyond $240 \mathrm{~nm}$, which was also in accordance with the results shown in Figures 12 through 15 . Therefore, only results from $190 \mathrm{~nm}$ to $250 \mathrm{~nm}$ are presented in the absorbance spectra for this phase of the experiment. 
(a) High Concentration Range

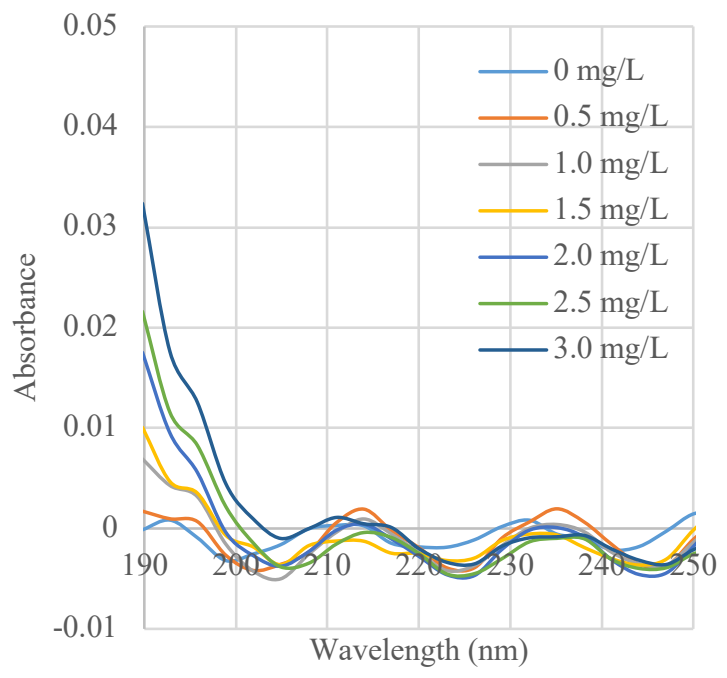

(b) Low Concentration Range

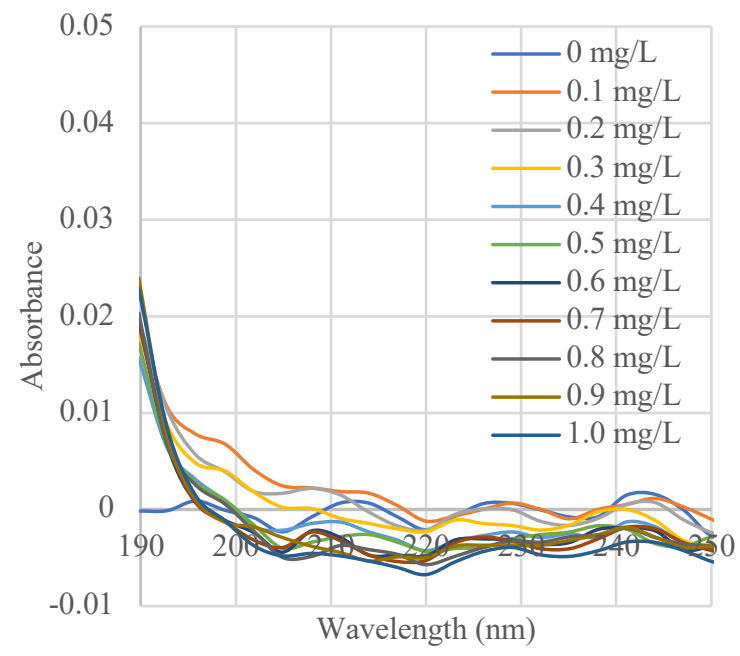

Figure 13: Absorbance spectra of Magnafloc LT27AG polymer in deionized water using an in-line spectrophotometer with a $2 \mathrm{~mm}$ flow cell in the concentration ranges of (a) $0-3 \mathrm{mg} / \mathrm{L}$ and (b) $0-1 \mathrm{mg} / \mathrm{L}$

(a) High Concentration Range

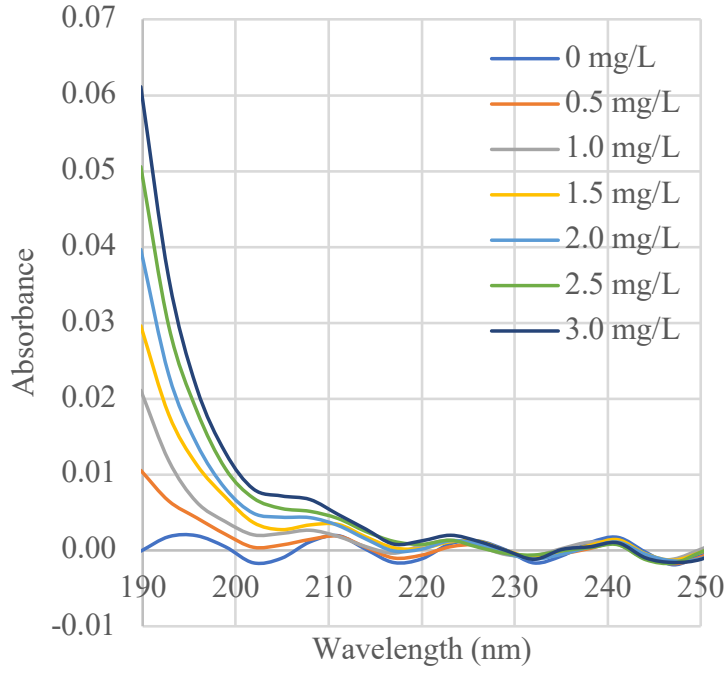

(b) Low Concentration Range

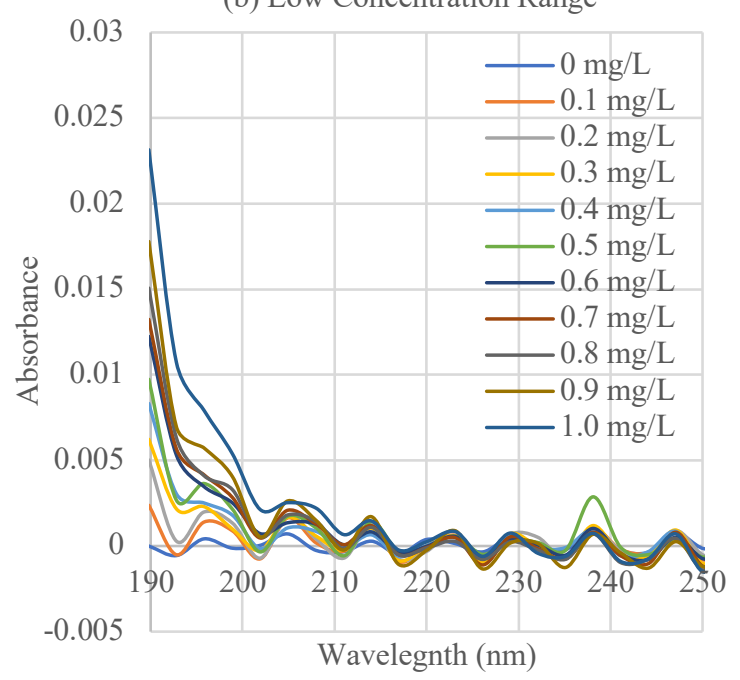

Figure 14: Absorbance spectra of Magnafloc LT27AG polymer in deionized water using an in-line spectrophotometer with a $4 \mathrm{~mm}$ flow cell in the concentration ranges of (a) $0-3 \mathrm{mg} / \mathrm{L}$ and (b) $0-1 \mathrm{mg} / \mathrm{L}$ 
(a) High Concentration Range

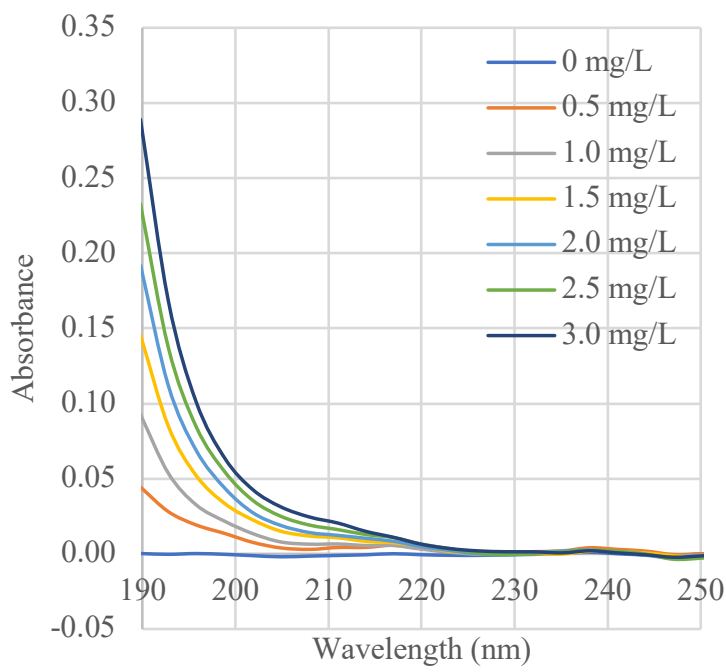

(b) Low Concentration Range

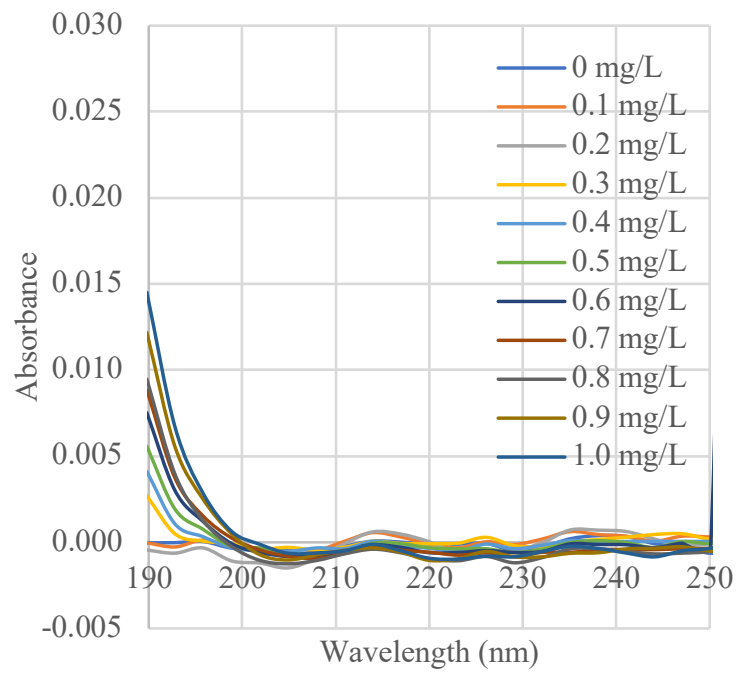

Figure 15: Absorbance spectra of Magnafloc LT27AG polymer in deionized water using an in-line spectrophotometer with an $8 \mathrm{~mm}$ flow cell in the concentration ranges of (a) $0-3 \mathrm{mg} / \mathrm{L}$ and (b) $0-1 \mathrm{mg} / \mathrm{L}$

Figures 12 through 15 demonstrate that as the concentration of Magnafloc LT27AG polymer was increased, the corresponding absorbance reading also increased. This was true for both the desktop and in-line spectrophotometers at the $2 \mathrm{~mm}, 4 \mathrm{~mm}, 8 \mathrm{~mm}$ and $10 \mathrm{~mm}$ path lengths for both the high and low polymer concentration ranges. The highest absorbance value in all cases was measured at $190 \mathrm{~nm}$, which is in accordance with a previous study performed by Al Momani and Örmeci (22), which found a maximum absorbance value of polymer in a variety of water matrices (sludge centrate, raw water, clarified water and distilled water) to occur at $191.5 \mathrm{~nm}$ when using an in-line UV-vis spectrophotometer. As the wavelength increases, the absorbance values decrease, tending to hover around zero with no significant absorbance readings beyond $240 \mathrm{~nm}$ between the variable polymer concentrations.

Upon firstly analyzing the shape of the absorbance spectra for the high concentration range, it is clear from Figure 12a that the $2 \mathrm{~mm}$ path length in the in-line spectrophotometer yields 
a quite turbulent absorbance spectra when compared to the other path lengths. Figures 13a and $15 \mathrm{a}$ demonstrate that as the path length was increased, the variance in absorbance became smoother and more linear. There was also less noise at the higher wavelengths when the path length was increased, as can be observed when comparing Figure 13a to 15a. The same is true for the shape of the absorbance spectra at the low concentration range, as can be see when comparing Figure $13 \mathrm{~b}$ to Figures $14 \mathrm{~b}$ and $15 \mathrm{~b}$. The shape of the absorbance spectra curve produced by the desktop unit showed very similar results, however the unit did always pick up some background around 254nm, consistent with the wavelength at which organic matter absorbs light the most effectively (49) (50). It should be noted that the desktop unit is a double-beam UV-vis spectrophotometer, meaning the light-source can be diffracted through both the sample cell and a reference cell containing a sample solution containing no polymer. The absorbance attributed to the reference cell is measured simultaneously and is then subtracted as background from the overall reading attributed to the sample cell, which then reports the absorbance value attributed to the polymer only. As the quartz cells used for the purpose of this experiment had been used previously for other experiments, there is the possibility that some residual organic matter from previous experiments was permanently present on the cell and was therefore not removed as background, which would explain the slight peak around 254nm.

Analyzing the magnitude of the absorbance values at $190 \mathrm{~nm}$ for each concentration between path lengths on the in-line unit at the high concentration range, it can be observed from Figure 15a that the $8 \mathrm{~mm}$ path length was able to detect the highest absorbance readings for every different polymer concentration sample. The results show that as the 
path length increased, the absorbance values for each polymer concentration increased as well. In addition to this, it is also notable that as the path length increased, the incremental difference in absorbance readings between polymer concentrations increased as well. Table 5 below summarizes this absorbance spectra data for the high concentration range. Although it would appear that the $8 \mathrm{~mm}$ path length is most sensitive, it is also important to take into consideration the standard deviation between the incremental differences in absorbance. A larger standard deviation would indicate a less linear relationship between polymer concentration and absorbance at $190 \mathrm{~nm}$. From the standard deviation of these values indicated in Table 5, it is apparent that at the increase in absorbance occurring using the $8 \mathrm{~mm}$ path length deviates most of the three path length variations, suggesting the absorbance-concentration relationship may be weaker for this path length when compared to the $4 \mathrm{~mm}$ path length. The $2 \mathrm{~mm}$ path length yielded the lowest standard deviation between absorbance increments, however it also was not able to detect a very strong absorbance when compared to the other path lengths and the shape of the absorbance spectra was very turbulent. When comparing the in-line results to the desktop unit results at the high concentration range, a background of approximately 0.300 A.U. is present due to the double-beam technology explained previously. In order to compare these values accurately with the in-line unit values, this background absorbance was subtracted from each of the sample absorbance values and the differences are listed in Table 5 below. From the desktop spectrophotometer results, it can be determined that the $10 \mathrm{~mm}$ path length flow cell in the desktop unit was able to detect a maximum absorbance for each concentration higher than the $4 \mathrm{~mm}$ path length and lower than the $8 \mathrm{~mm}$ path length, however quite comparable to the two. The average incremental increase of 0.0215 A.U. was also in 
between that of the $4 \mathrm{~mm}$ and $8 \mathrm{~mm}$ path lengths using the in-line unit, which were 0.0102 A.U. and 0.0481 A.U. respectively. The standard deviation of the incremental absorbance measurements was 0.0052 A.U., comparable to that of the $8 \mathrm{~mm}$ path length which was 0.0058 A.U.

When analyzing the magnitude of the absorbance values at $190 \mathrm{~nm}$ for each concentration between path lengths on the in-line unit at the low concentration range, it can be observed from Figure $12 \mathrm{~b}$ that the $4 \mathrm{~mm}$ path length was able to detect the highest absorbance values for each different polymer concentration sample. These results differ from those of the higher concentration range, which indicated that an increase in path length coordinated with an increase in absorbance value for a given concentration. In the case of the low concentration range, the $8 \mathrm{~mm}$ path length detected a lower absorbance reading than that of the $4 \mathrm{~mm}$ path length, as is observed when comparing Figure $14 \mathrm{~b}$ to Figure $15 \mathrm{~b}$. Table 6 below summarizes this absorbance spectra data for the low concentration range. From this data, it can be observed that the $4 \mathrm{~mm}$ path length also offered the largest average incremental increase in absorbance at $190 \mathrm{~nm}$ of 0.0023 A.U. between the different concentrations of polymer. The standard deviation of 0.0016 A.U. between these values was quite low as well. The $8 \mathrm{~mm}$ path length showed a decrease in average incremental absorbance at $190 \mathrm{~nm}$ with a value of 0.0015 A.U. when compared to the $4 \mathrm{~mm}$ path length. This data suggests that at very low polymer concentrations, the $4 \mathrm{~mm}$ path length actually performed better than the $8 \mathrm{~mm}$ path length. When comparing these results to that of the desktop unit in the low concentration range, the results are very consistent with that of the $4 \mathrm{~mm}$ path length in the in-line unit, with an average incremental absorbance increase at 
190nm of 0.004 A.U., associated with a standard deviation of 0.003 A.U. Table 6 shows that at very low concentrations, however, the desktop unit appears to be far less sensitive than the in-line unit. The average incremental absorbance values at $190 \mathrm{~nm}$ between $0 \mathrm{mg} / \mathrm{L}$ of polymer up to $1.0 \mathrm{mg} / \mathrm{L}$ fluctuates from a minimum of 0.019 A.U. to 0.093 A.U., indicating that the relationship between concentration and absorbance is less linear. When comparing these values to that of the in-line unit using the $4 \mathrm{~mm}$ path length, the average incremental increase in absorbance at $190 \mathrm{~nm}$ remains far steadier with a minimum of 0.0010 A.U. and a maximum of 0.0027 A.U. The standard deviation of these values between the in-line unit and the desktop unit of 0.0016 A.U. and 0.0027 A.U., respectively, further indicates this point. 


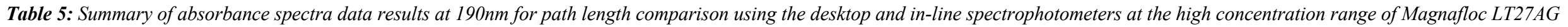
polymer $(0-3 \mathrm{mg} / \mathrm{L})$

\begin{tabular}{|c|c|c|c|c|c|c|c|c|c|}
\hline \multirow{3}{*}{ Concentration } & \multicolumn{3}{|c|}{ Desktop Unit 10mm path length } & \multicolumn{2}{|c|}{ In-line Unit $2 \mathrm{~mm}$ path length } & \multicolumn{2}{|c|}{ In-line Unit 4mm path length } & \multicolumn{2}{|c|}{ In-line Unit 8mm path length } \\
\hline & Raw & Absorbance & Incremental & Absorbance & Incremental & Absorbance & Incremental & Absorbance & Incremental \\
\hline & Absorbance & Less & Absorbance & & Absorbance & & Absorbance & & Absorbance \\
\hline 0.0 & 0.2902 & 0.0000 & & -0.00013 & & -0.00010 & & 0.00025 & \\
\hline 0.5 & 0.3167 & 0.0265 & 0.0265 & 0.00172 & 0.0019 & 0.01055 & 0.0106 & 0.04445 & 0.0442 \\
\hline 1.5 & 0.3573 & 0.0671 & 0.0251 & 0.01007 & 0.0031 & 0.02959 & 0.0085 & 0.14560 & 0.0529 \\
\hline 2.0 & 0.3733 & 0.0830 & 0.0160 & 0.01753 & 0.0075 & 0.03966 & 0.0101 & 0.19218 & 0.0466 \\
\hline 2.5 & 0.3999 & 0.1097 & 0.0266 & 0.02161 & 0.0041 & 0.05057 & 0.0109 & 0.23270 & 0.0405 \\
\hline \multirow[t]{6}{*}{3.0} & 0.4194 & 0.1292 & 0.0195 & 0.03237 & 0.0108 & 0.06111 & 0.0105 & 0.28907 & 0.0564 \\
\hline & & Average & & Average & & Average & & Average & \\
\hline & & Incremental & 0.0215 & Incremental & 0.0054 & Incremental & 0.0102 & Incremental & 0.0481 \\
\hline & & STDV of & & STDV of & & STDV of & & STDV of & \\
\hline & & Incremental & 0.00519 & Incremental & 0.00324 & Incremental & 0.00088 & Incremental & 0.00576 \\
\hline & & Increase & & Increase & & Increase & & Increase & \\
\hline
\end{tabular}




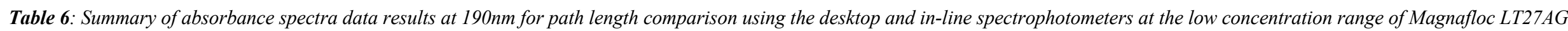
polymer $(0-1 \mathrm{mg} / \mathrm{L})$

\begin{tabular}{|c|c|c|c|c|c|c|c|c|c|}
\hline \multirow{3}{*}{ Concentration } & \multicolumn{3}{|c|}{ Desktop Unit 10mm path length } & \multicolumn{2}{|c|}{ In-line Unit $2 \mathrm{~mm}$ path length } & \multicolumn{2}{|c|}{ In-line Unit 4mm path length } & \multicolumn{2}{|c|}{ In-line Unit 8mm path length } \\
\hline & Raw & Absorbance & Incremental & Absorbance & Incremental & Absorbance & Incremental & Absorbance & Incremental \\
\hline & Absorbance & Less & Absorbance & & Absorbance & & Absorbance & & Absorbance \\
\hline $\mathbf{0 . 0}$ & 0.29162 & 0.0000 & & -0.00016 & & 0.00000 & & -0.00003 & \\
\hline 0.1 & 0.29521 & 0.00358 & 0.0036 & 0.01952 & 0.0197 & 0.00238 & 0.0024 & -0.00003 & 0.0000 \\
\hline 0.3 & 0.29634 & 0.00471 & 0.0031 & 0.01856 & 0.0009 & 0.00623 & 0.0012 & 0.00270 & 0.0032 \\
\hline 0.4 & 0.30287 & 0.01124 & 0.0065 & 0.01571 & 0.0028 & 0.00832 & 0.0021 & 0.00412 & 0.0014 \\
\hline 0.5 & 0.29914 & 0.00751 & 0.0037 & 0.01725 & 0.0015 & 0.00973 & 0.0014 & 0.00557 & 0.0015 \\
\hline 0.6 & 0.30270 & 0.01107 & 0.0036 & 0.01975 & 0.0025 & 0.01224 & 0.0025 & 0.00752 & 0.0020 \\
\hline 0.7 & 0.30608 & 0.01446 & 0.0034 & 0.01929 & 0.0005 & 0.01324 & 0.0010 & 0.00886 & 0.0013 \\
\hline 0.8 & 0.30395 & 0.01233 & 0.0021 & 0.02031 & 0.0010 & 0.01507 & 0.0018 & 0.00947 & 0.0006 \\
\hline \multirow[t]{7}{*}{1.0} & 0.32023 & 0.02861 & 0.0070 & 0.02292 & 0.0011 & 0.02315 & 0.0000 & 0.01451 & 0.0023 \\
\hline & & Average & & Average & & Average & & Average & \\
\hline & & Incremental & 0.0044 & Incremental & 0.0034 & Incremental & 0.0018 & Incremental & 0.0015 \\
\hline & & Increase & & Increase & & Increase & & Increase & \\
\hline & & STDV of & & STDV of & & STDV of & & STDV of & \\
\hline & & Incremental & 0.00271 & Incremental & 0.00118 & Incremental & 0.00101 & Incremental & 0.00076 \\
\hline & & Increase & & Increase & & Increase & & Increase & \\
\hline
\end{tabular}




\subsubsection{Linear correlations}

The results from Figures 12 through 15 above demonstrate that the absorbance values at a 190nm wavelength were highest for all samples on both the in-line spectrophotometer and the desktop spectrophotometer. This is in accordance with a previous study performed by Al Momani and Örmeci (22), which found a maximum absorbance value of polymer in a variety of water matrices (sludge centrate, raw water, clarified water and distilled water) to occur at $191.5 \mathrm{~nm}$ when using an in-line UV-vis spectrophotometer. In order to further analyze this relationship and determine which path length offered the most sensitive results, a linear correlation of the absorbance-polymer concentration data was done for all datasets. Figures $16 \mathrm{a}$ and $16 \mathrm{~b}$ below represent the positive linear correlation between the polymer concentration and absorbance readings using the desktop spectrophotometer at the $0-3$ $\mathrm{mg} / \mathrm{L}$ and the $0-1 \mathrm{mg} / \mathrm{L}$ concentration ranges respectively. The polymer concertation for these and all subsequent linear correlations refers to the dosed concentration in the sample.

(a) High Concentration Range

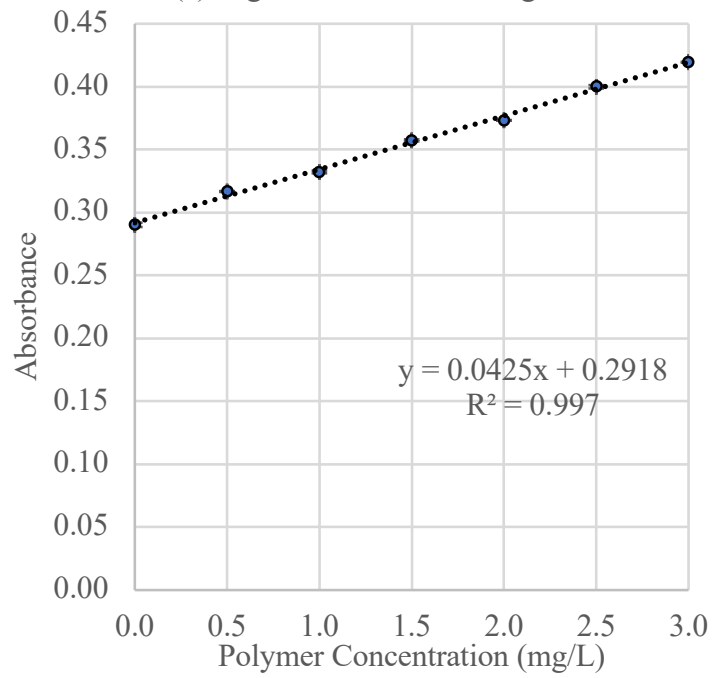

(b) Low Concentration Range

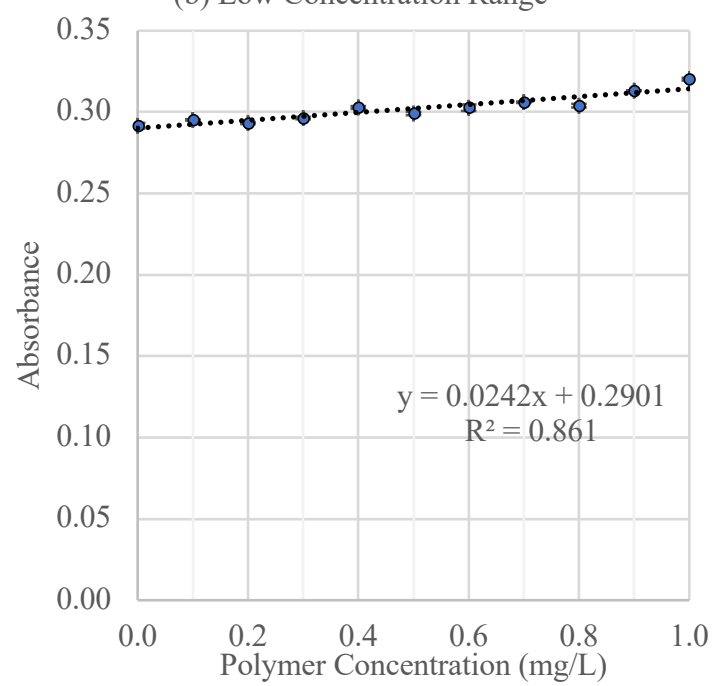

Figure 16: Linear correlation of the absorbance-polymer concentration data of Magnafloc LT27AG in deionized water at 190nm using a desktop spectrophotometer with a $10 \mathrm{~mm}$ flow cell in the concentration ranges of (a) $0-3 \mathrm{mg} / \mathrm{L}$ and (b) $0-1 \mathrm{mg} / \mathrm{L}$ 
Figures $17 \mathrm{a}$ and $17 \mathrm{~b}$ represent the linear correlation of the absorbance-polymer concentration data using the in-line spectrophotometer and the $2 \mathrm{~mm}$ flow cell at the 0 $\mathrm{mg} / \mathrm{L}$ and $0-1 \mathrm{mg} / \mathrm{L}$ polymer concentration ranges respectively. Again, the absorbancepolymer concentration data was plotted using this same linear correlation method for both the $4 \mathrm{~mm}$ and $8 \mathrm{~mm}$ flow cells using the in-line spectrophotometer. Figures $18 \mathrm{a}$ and $18 \mathrm{~b}$ below represent this data using the $4 \mathrm{~mm}$ flow cell at both the high and low concentration ranges, respectively, and Figures $19 \mathrm{a}$ and $19 \mathrm{~b}$ represent the $8 \mathrm{~mm}$ flow cell at both the high and low concentration ranges, respectively.

(a) High Concentration Range

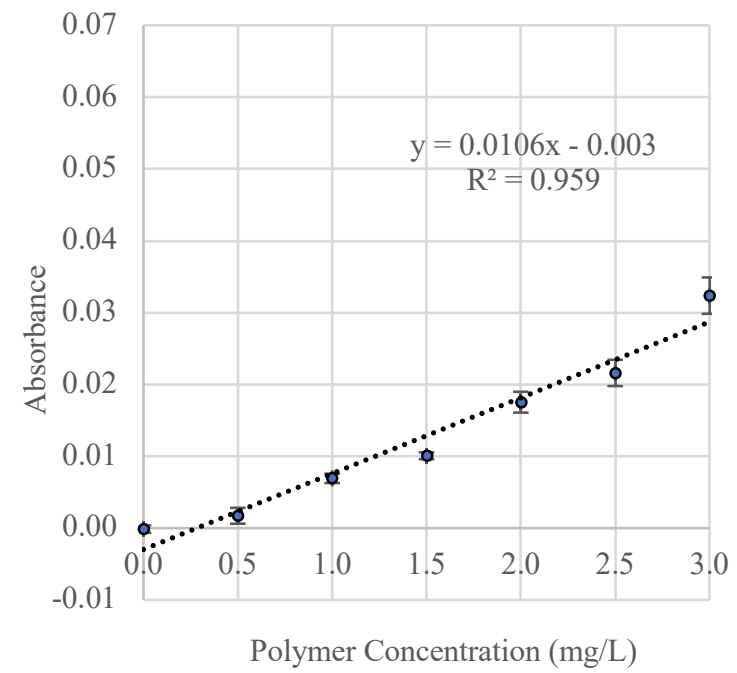

(b) Low Concentration Range

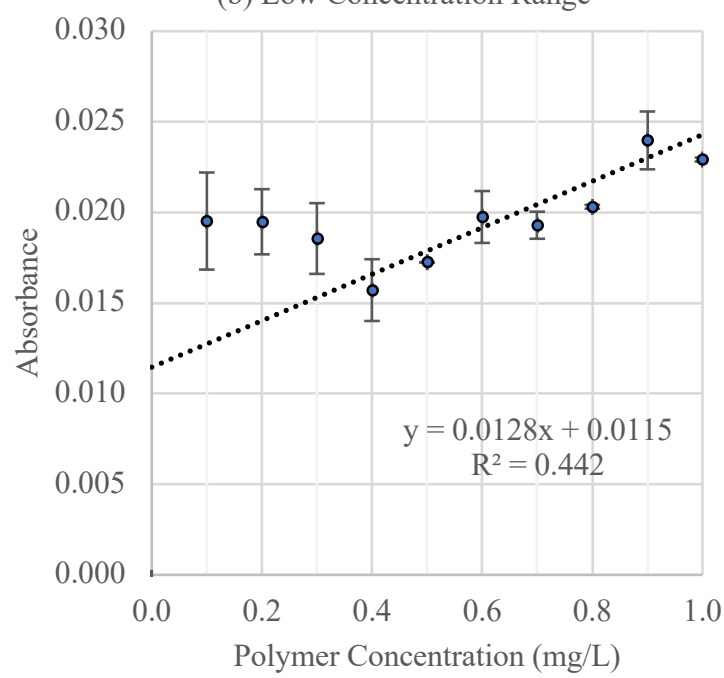

Figure 17: Linear correlation of the absorbance-polymer concentration data of Magnafloc LT27AG in deionized water at 190nm using an in-line spectrophotometer with a $2 \mathrm{~mm}$ flow cell in the concentration ranges of (a) 0-3 $\mathrm{mg} / \mathrm{L}$ and (b) $0-1 \mathrm{mg} / \mathrm{L}$ 
(a) High Concentration Range

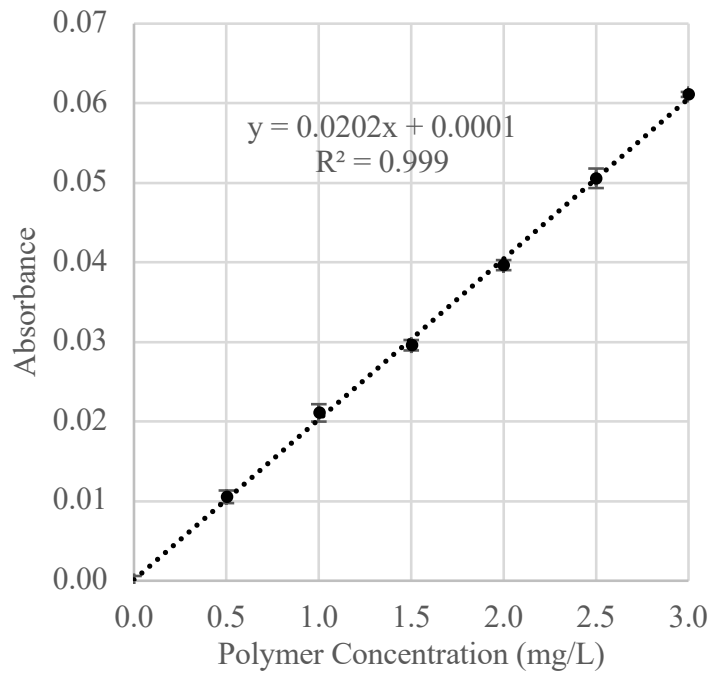

(b) Low Concentration Range

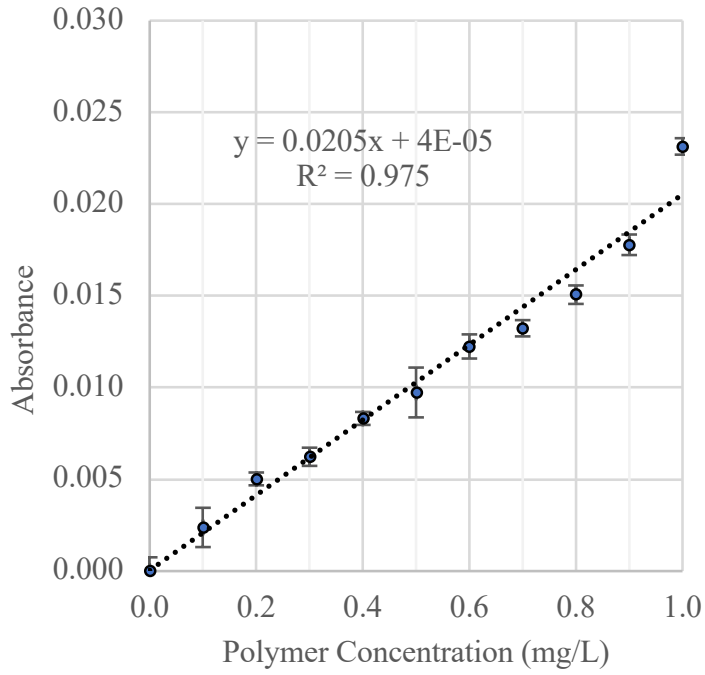

Figure 18: Linear correlation of the absorbance-polymer concentration data of Magnafloc LT27AG in deionized water at 190nm using an in-line spectrophotometer with a $4 \mathrm{~mm}$ flow cell in the concentration ranges of (a) 0-3 $\mathrm{mg} / \mathrm{L}$ and (b) $0-1 \mathrm{mg} / \mathrm{L}$

(a) High Concentration Range

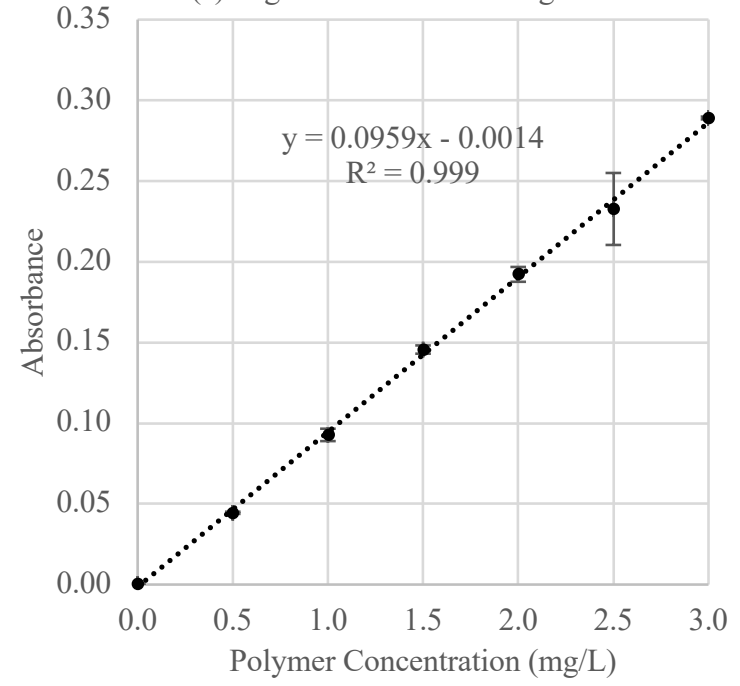

(b) Low Concentration Range

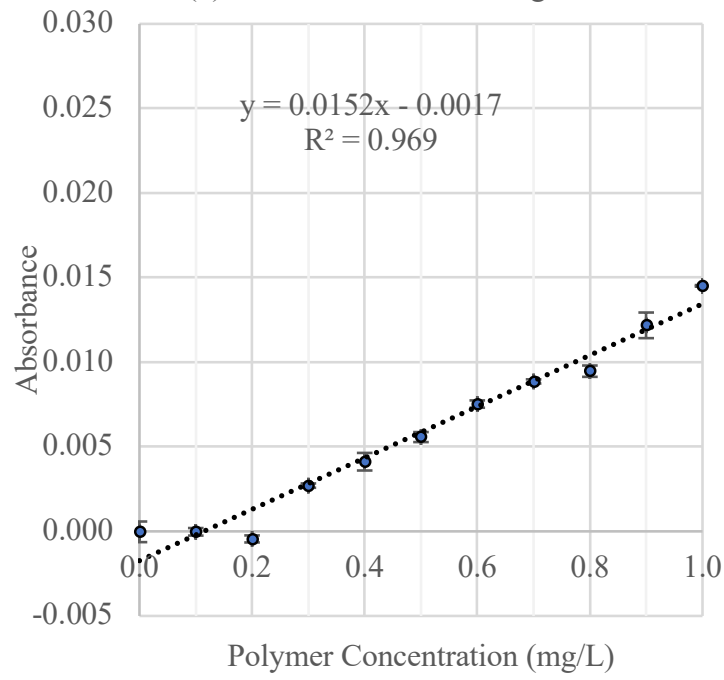

Figure 19: Linear correlation of the absorbance-polymer concentration data of Magnafloc LT27AG in deionized water at 190nm using an in-line spectrophotometer with an $8 \mathrm{~mm}$ flow cell in the concentration ranges of (a) 0-3 mg/L and (b) $0-1 \mathrm{mg} / \mathrm{L}$ 
Figures 16 through 19 demonstrate that at a $190 \mathrm{~nm}$ wavelength, UV-vis spectroscopy is capable of demonstrating a quite strong linear correlation between the polymer concentration in a given solution and its absorbance reading at both high and low concentration ranges. All linear correlations depicted in Figures 16 though 19 denote an incremental increase in absorbance readings as the polymer concentration increases in solution with deionized water. These results are consistent with those reported by a similar study done by Al Momani and Örmeci, as well as Gibbons and Örmeci (22).

Upon analysis of the linear correlation curves of the desktop and in-line UV-vis spectrophotometers, some comparisons can be made. Both spectrophotometers were able to form very strong linear correlations $\left(\mathrm{R}^{2}>0.99\right)$ between the absorbance values and the polymer concentration in the high concentration range (0-3 mg/L), as depicted in Figures 16a, 17a, 18a and 19a. From the linear correlation curve in Figure 16b, it is apparent that the desktop spectrophotometer is less sensitive in detecting polymer in the low concentration range $(0-1 \mathrm{mg} / \mathrm{L})$ than the in-line spectrophotometer based on the $\mathrm{R}^{2}$ value of 0.861 A.U. and the quite low slope of 0.0242 . Figures $18 \mathrm{~b}$ and $19 \mathrm{~b}$ demonstrate that the in-line unit was able to form quite a strong linear correlation $\left(\mathrm{R}^{2}=0.97\right)$ between polymer concentration and absorbance values for the low concentration range using the $4 \mathrm{~mm}$ and $8 \mathrm{~mm}$ flow cells, whereas the desktop unit was not. At the higher concentration range (0-3 $\mathrm{mg} / \mathrm{L}$ ), however, the desktop spectrophotometer was capable of detecting the full range of concentrations and formed a strong linear correlation $\left(\mathrm{R}^{2}=0.997\right)$. A full summary of the coefficient of determinations can be observed below in Table 7 . 
It was previously noted that the desktop unit is a double beam spectrophotometer, meaning the light-source can be diffracted through both the sample cell and a reference cell containing a sample solution containing no polymer, which is then subtracted from the sample reading as background. As there are variances between the flow cell used for the sample and the flow cell used for reference, the zero value that is reported for the desktop unit hovers around an approximate value of 0.300 A.U. For the purpose of comparing the in-line unit to the desktop unit, this value was subtracted from all maximum absorbance values reported by the desktop unit at 190nm and are listed above in Tables 5 and 6 . For the purpose of comparison between spectrophotometers, these absorbance values will be used. When comparing these values, the desktop unit and the in-line unit are in agreeance at the low concentration range, with a maximum absorbance value at $1.0 \mathrm{mg} / \mathrm{L}$ of $\sim 0.020$ A.U., as is visible in Table 6. At the higher concentration however, the desktop unit was able to detect a stronger absorbance reading for the $3.0 \mathrm{mg} / \mathrm{L}$ than that of the in-line unit using the $2 \mathrm{~mm}$ and $4 \mathrm{~mm}$ flow cells, as is visible in Table 5 . When the $8 \mathrm{~mm}$ flow cell was used, the in-line unit was able to detect a much higher absorbance value at the $3.0 \mathrm{mg} / \mathrm{L}$ concentration of 0.289 A.U.

As was previously mentioned, the in-line unit is capable of utilizing different flow cells, each with a different path length. The path length is a critical parameter in the BeerLambert law stated in equation 5, which is the main governing principle of UV-vis spectroscopy. The path length was varied between $2 \mathrm{~mm}, 4 \mathrm{~mm}$ and $8 \mathrm{~mm}$ using the in-line unit for both the high concentration sample range and the low concentration sample range in order to analyze and compare absorbance values and sensitivity between path lengths. 
The $2 \mathrm{~mm}$ path length was not effective in detecting low concentrations of polymer, offering a weak linear correlation $\left(\mathrm{R}^{2}=0.442\right)$ as can be seen in Figure $17 \mathrm{~b}$. The $4 \mathrm{~mm}$ and $8 \mathrm{~mm}$ path lengths were able to form much stronger linear correlations, both with $\mathrm{R}^{2}$ values of $\sim 0.970$, as depicted in Figures $18 \mathrm{~b}$ and 19b. Tables 7 and 8 below depicts a full summary of the coefficient of determinations at both the high and low polymer concentration range, respectively.

Table 7: Summary of coefficient of determinations for desktop and in-line UV-vis spectrophotometers at a high Magnafloc LT27AG polymer concentration range $(0-3 \mathrm{mg} / \mathrm{L})$

\begin{tabular}{lcc}
\hline \multicolumn{1}{c}{ UV-vis spectrophotometer model } & Path length (mm) & ${\text { Coefficient of determination } \mathbf{R}^{\mathbf{2}}}^{\text {(mesktop }}$ \\
\hline In-Line & 10 & 0.997 \\
\hline In-Line & 2 & 0.959 \\
\hline In-Line & 4 & 0.999 \\
\hline
\end{tabular}

Table 8: Summary of coefficient of determinations for desktop and in-line UV-vis spectrophotometers at a low Magnafloc LT27AG polymer concentration range $(0-1 \mathrm{mg} / \mathrm{L})$

\begin{tabular}{lcc}
\hline \multicolumn{1}{c}{ UV-vis spectrophotometer model } & Path length $(\mathbf{m m})$ & Coefficient of determination $\mathbf{R}^{\mathbf{2}}$ \\
\hline Desktop & 10 & 0.861 \\
\hline In-Line & 2 & 0.442 \\
\hline In-Line & 4 & 0.975 \\
\hline In-Line & 8 & 0.969 \\
\hline
\end{tabular}

In order to perform a full comparison of the path lengths and determine which is most sensitive for the detection of residual polymer in drinking water treatment, the absorbance readings themselves, shape of the absorbance spectra, slope of the linear correlation as well as the incremental increase between absorbance readings associated with each concentration were analyzed and compared against each other. At the high concentration 
range, the $4 \mathrm{~mm}$ path length did offer the lowest detection limit, however the maximum absorbance value, smooth absorbance spectra and large incremental absorbance increase at $190 \mathrm{~nm}$ demonstrated by the $8 \mathrm{~mm}$ path length yields the conclusion that it might offer the most sensitive results for the concentration range of $0-3 \mathrm{mg} / \mathrm{L}$. At the low concentration range, the detection limits of all path lengths using the in-line unit were marginally different. When comparing the shape of the absorbance spectra, it is clear that the $2 \mathrm{~mm}$ path length creates a very turbulent curve, deterring from its use in this case. The $4 \mathrm{~mm}$ and $8 \mathrm{~mm}$ path lengths both offer smoother curve shapes, however the $4 \mathrm{~mm}$ path length is capable of detecting a higher absorbance value for a given concentration at the $0-1 \mathrm{mg} / \mathrm{L}$ concentration range than the $8 \mathrm{~mm}$ path length. The average incremental increase between absorbance values at the $4 \mathrm{~mm}$ path length was also larger $(0.0010$ A.U.) than that of the 8mm path length (0.0007 A.U.), as stated in Table 7. When then comparing the $\mathrm{R}^{2}$ values of the absorbance-concentration linear correlations, the $4 \mathrm{~mm}$ path length was also slightly more linear $\left(\mathrm{R}^{2}=0.98\right)$ when compared to that of the $8 \mathrm{~mm}$ path length $\left(\mathrm{R}^{2}=0.97\right)$. The combination of these results infers that the $4 \mathrm{~mm}$ path length offered the most sensitive results at the low concentration range of $0-1 \mathrm{mg} / \mathrm{L}$ of polymer. Seeing that the maximum dosage limits for PAM polyelectrolytes in drinking water treatment is currently set to $<1.0$ $\mathrm{mg} / \mathrm{L}$ by the National Sanitation Foundation, the path length that is most sensitive for the detection of polymers at this low range was to be chosen for the remainder of the experiments, this being the $4 \mathrm{~mm}$ path length. 


\subsection{Effect of particles on polymer detection using $U V$-vis spectroscopy}

\subsubsection{UV-vis absorbance spectra}

The effect of suspended particulate matter present in surface waters on the sensitivity of UV-vis spectroscopy as a method for the detection of residual polymer was assessed at both a high concentration range of Magnafloc LT27AG polymer $(0-3 \mathrm{mg} / \mathrm{L})$ as well as a low concentration range $(0-1 \mathrm{mg} / \mathrm{L})$. Water samples collected from the Rideau River in Ottawa, Ontario, Canada were prepared in four different methods: filtered through a $0.45 \mu \mathrm{m}$ filter, diluted (1:1) with deionized water, both filtered and diluted (1:1) with deionized water, as well as unfiltered and undiluted. These prepared samples were spiked with polymer at both the high and low concentration ranges and were analyzed using both the desktop spectrophotometer using a $10 \mathrm{~mm}$ path length quartz flow cell, and the in-line spectrophotometer using a $4 \mathrm{~mm}$ path length flow cell. Results between the four types of prepared samples were then compared to establish the effect of particulate matter present in the sample on the sensitivity of UV-vis spectroscopy as a detection method for residual polymer, as well as to compare this sensitivity between both the desktop and in-line spectrophotometers. Figures 20a and $20 \mathrm{~b}$ below represent the absorbance spectra for the 0 $3.0 \mathrm{mg} / \mathrm{L}$ and $0-1.0 \mathrm{mg} / \mathrm{L}$ polymer concentration ranges respectfully using the desktop spectrophotometer in an unfiltered and undiluted Rideau River water sample. Figures 21a and $21 \mathrm{~b}$ below represent the absorbance spectra for the $0-3 \mathrm{mg} / \mathrm{L}$ and $0-1 \mathrm{mg} / \mathrm{L}$ polymer concentration ranges respectfully using a $4 \mathrm{~mm}$ path length flow cell and the in-line spectrophotometer in the same unfiltered and undiluted Rideau River water sample. As the change in absorbance is very small between polymer concentrations, Figures 20 and 21 
below include a smaller scale graph overlaid to demonstrate the incremental increases in absorbance, although very minor.

(a) High Concentration Range

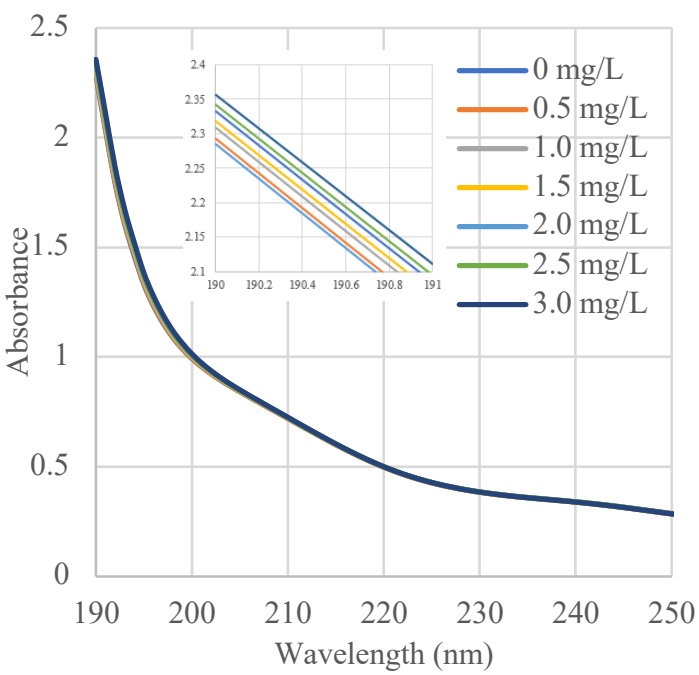

(b) Low Concentration Range

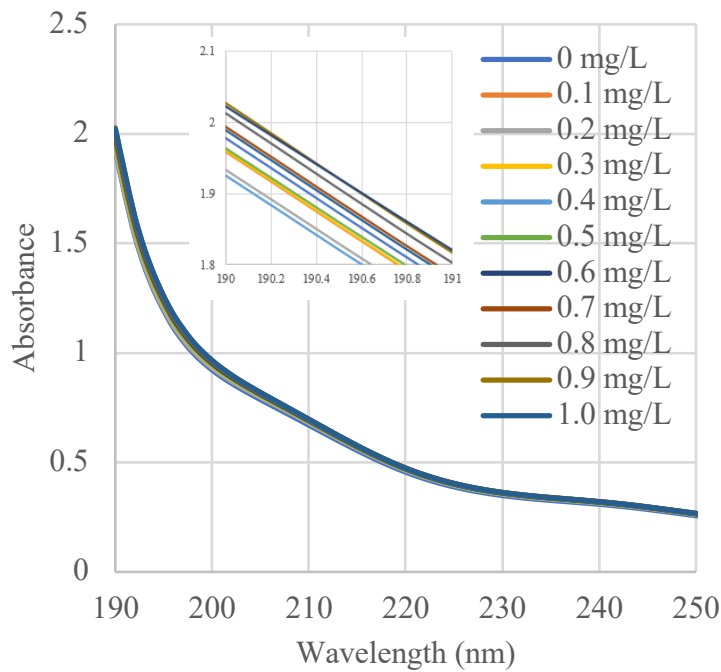

Figure 20: Absorbance spectra of Magnafloc LT27AG polymer in unfiltered and undiluted Rideau River water with a turbidity of 6.8NTU using a desktop spectrophotometer with a $10 \mathrm{~mm}$ flow cell in the concentration ranges of (a) 0-3 $\mathrm{mg} / \mathrm{L}$ and (b) $0-1 \mathrm{mg} / \mathrm{L}$

(a) High Concentration Range

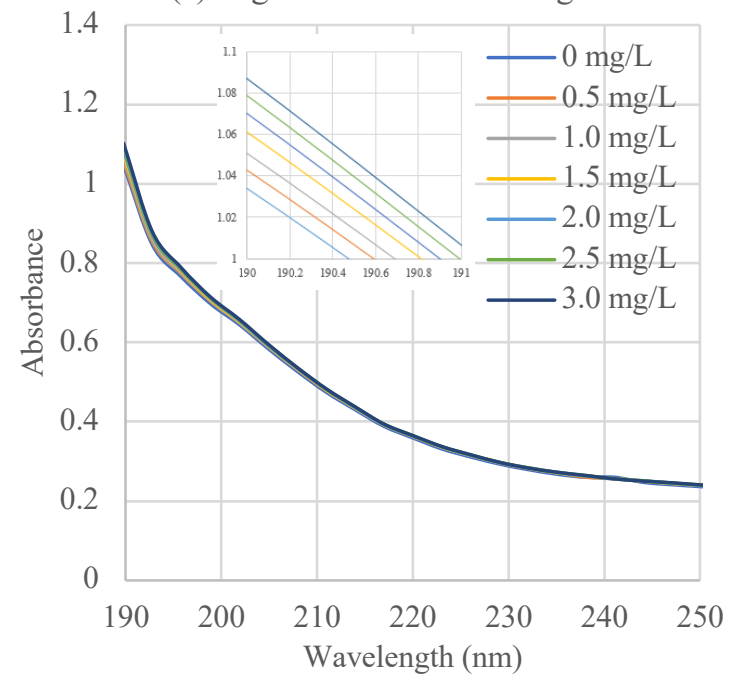

(b) Low Concentration Range

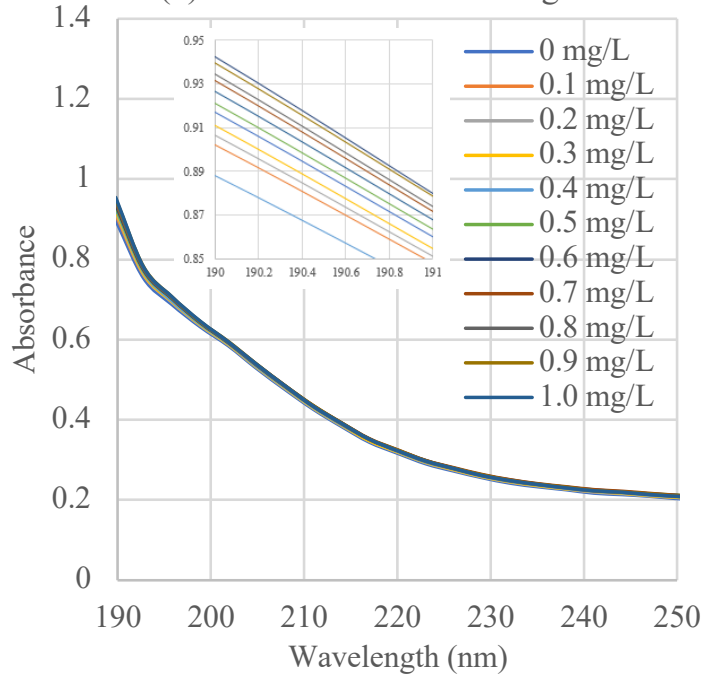

Figure 21: Absorbance spectra of Magnafloc LT27AG polymer in unfiltered and undiluted Rideau River water with a turbidity of 6.8NTU using an in-line spectrophotometer with a $4 \mathrm{~mm}$ flow cell in the concentration ranges of (a) 0-3 mg/L and (b) $0-1 \mathrm{mg} / \mathrm{L}$ 
A portion of the same bulk Rideau River water sample was then filtered through a $0.45 \mu \mathrm{m}$ filter on a different date, and the polymer concentrations were once again dosed at both the high and low concentration ranges. The raw Rideau River water sample had a turbidity of 6.8NTU, however after filtration this turbidity measured $0.15 \mathrm{NTU}$. The purpose of the filtration was to remove suspended particulate matter present in the water and observe the effect this had on the sensitivity of UV-vis spectroscopy for the detection of residual polymer. Once again, the samples at both the high and low concentration ranges were analyzed using both the desktop and in-line UV-vis spectrophotometers. Figures 22a and $22 \mathrm{~b}$ below represent the absorbance spectra for the $0-3 \mathrm{mg} / \mathrm{L}$ and $0-1 \mathrm{mg} / \mathrm{L}$ polymer concentration ranges respectfully using a $10 \mathrm{~mm}$ path length flow cell and the desktop spectrophotometer in a filtered and undiluted Rideau River water sample.

(a) High Concentration Range

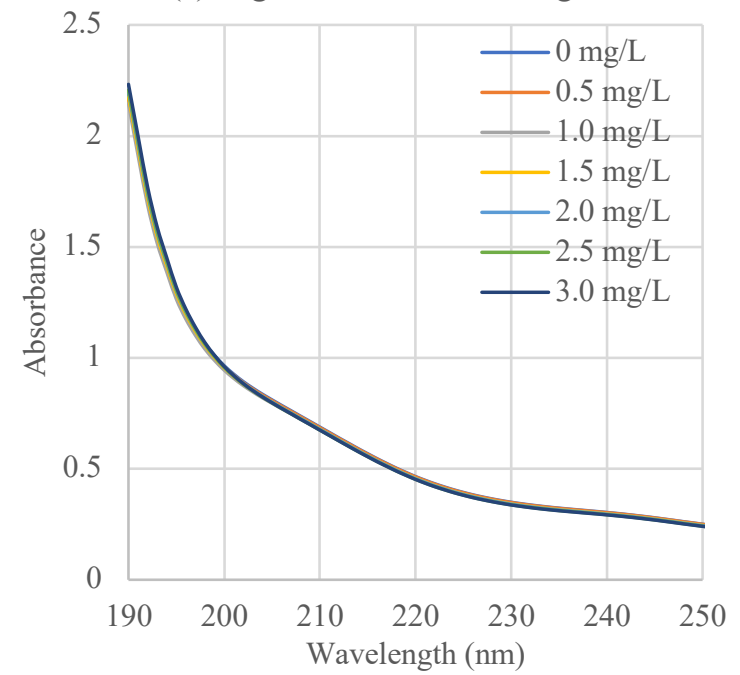

(b) Low Concentration Range

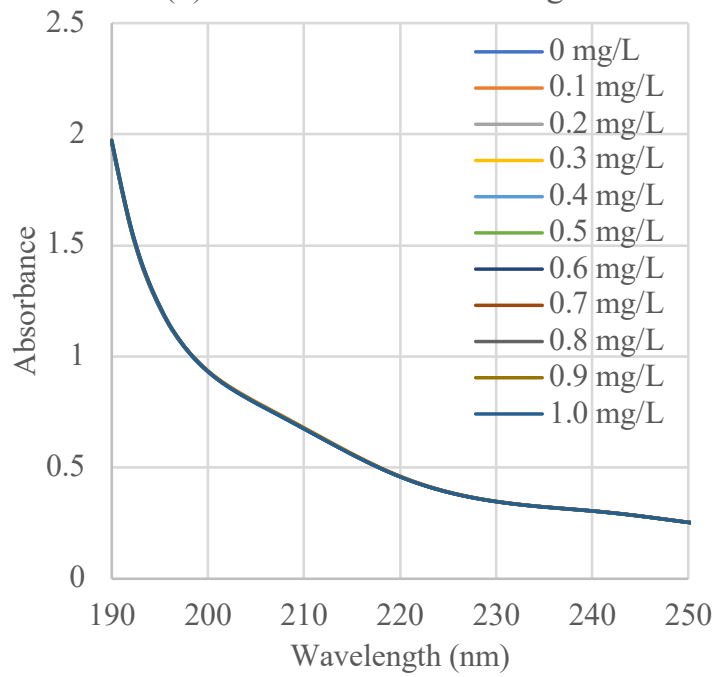

Figure 22: Absorbance spectra of Magnafloc LT27AG polymer in filtered (0.45 4 ) and undiluted Rideau River water with an initial turbidity of $6.8 N T U$ using a desktop spectrophotometer with a $10 \mathrm{~mm}$ flow cell in the concentration ranges of (a) $0-3 \mathrm{mg} / \mathrm{L}$ and (b) $0-1 \mathrm{mg} / \mathrm{L}$ 
Figures $23 \mathrm{a}$ and $23 \mathrm{~b}$ below represent the absorbance spectra for the $0-3 \mathrm{mg} / \mathrm{L}$ and $0-1$ $\mathrm{mg} / \mathrm{L}$ polymer concentration ranges respectfully using a $4 \mathrm{~mm}$ path length flow cell and the in-line spectrophotometer in the same filtered $(0.45 \mu \mathrm{m})$ and undiluted Rideau River water sample.

(a) High Concentration Range

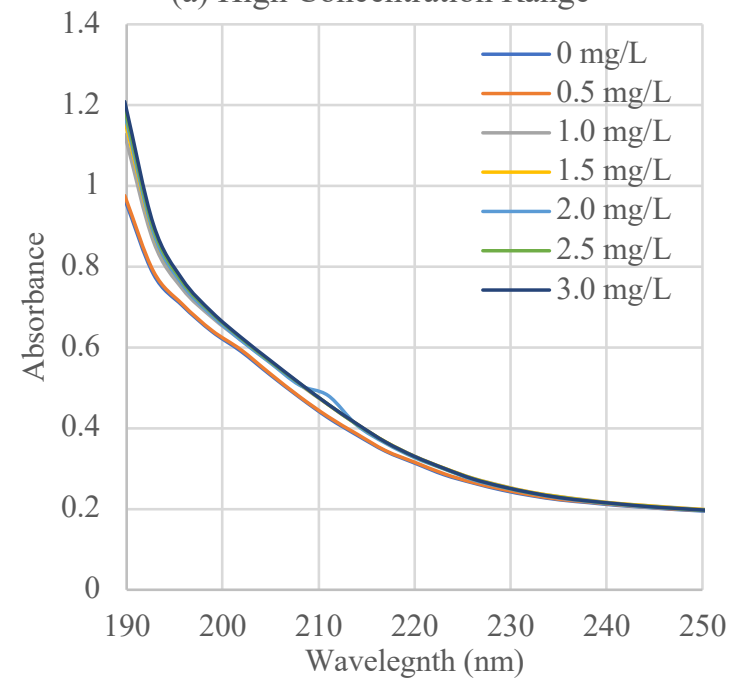

(b) Low Concentration Range

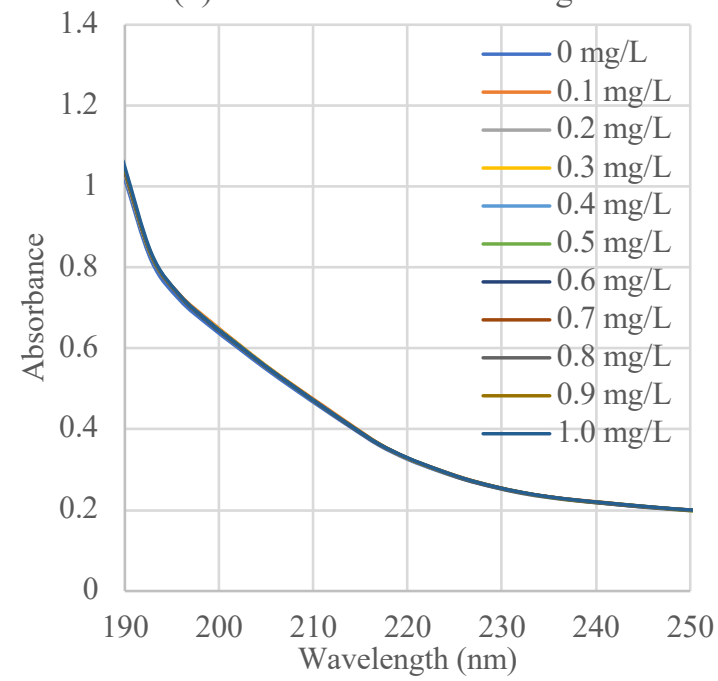

Figure 23: Absorbance spectra of Magnafloc LT27AG polymer in filtered (0.45 $\mu \mathrm{m})$ and undiluted Rideau River water with an initial turbidity of $6.8 N T U$ using an in-line spectrophotometer with a 4 mm flow cell in the concentration ranges of (a) $0-3 \mathrm{mg} / \mathrm{L}$ and (b) $0-1 \mathrm{mg} / \mathrm{L}$

Figures 20 and 21 demonstrate that the presence of particulate matter in the raw water sample did not affect the ability of UV-vis spectroscopy to detect residual polymer at 190nm. The absorbance spectra for these samples using both the in-line and desktop units followed the expected trend and maintained the expected shape. Figure 20 did indicate that the presence of particulate matter in the sample increased the maximum absorbance values quite substantially using the desktop unit at both the high and low concentration ranges when compared to that in the deionized water sample as seen in Figure 14. In deionized water, the $3.0 \mathrm{mg} / \mathrm{L}$ sample denoted an absorbance value of 0.4194 A.U. with the 
background included using the desktop spectrophotometer. The raw river water sample, however, read an absorbance value of 2.3568 A.U. using the same spectrophotometer, depicted in Figure 20a. The same increase was observed for the low concentration range as well, as can be seen in Figure 20b. When analyzing the absorbance results of the raw water samples read using the in-line unit (Figures 21a and 21b), an increase in absorbance was also observed in the presence of particles. Figures $21 \mathrm{a}$ and $21 \mathrm{~b}$ demonstrate that at both the high and low concentration ranges, the particulate matter present in the raw water sample caused the absorbance values at 190nm to increase toward 1.0 AU. Although these absorbance values are higher than those read in deionized water, they are still within the acceptable absorbance working range of 0 to $1.0 \mathrm{AU}$. This infers that the in-line unit would be better suited for detecting polymer in the presence of surface water particulate matter than the desktop unit, as the desktop unit would require substantial dilution to decrease the absorbance values within the acceptable working range.

Figures 22 and 23 demonstrate that post-filtration, the removal of particulate matter present in the raw surface water sample had a very marginal effect on the absorbance values reported at $190 \mathrm{~nm}$ by each spectrophotometer at both the high and low polymer concentration sample ranges. Filtered samples analyzed by the desktop unit, as represented by Figures $22 \mathrm{a}$ and $22 \mathrm{~b}$, resulted in absorbance spectra with an almost identical shape as the raw water samples, as represented by Figures 20a and 20b. When comparing the absorbance values between the filtered and raw samples at 190nm reported using the desktop unit, the filtered samples demonstrated little to no variation to the values reported by the raw water sample, indicating that particulate matter has little to no effect of the unit's 
ability to detect residual polymer. When comparing this to Figures 23a and 23b, representing the absorbance spectra of the filtered samples reported by the in-line spectrophotometer, very similar results are shown. Again, the shape of the curves at both the high and low remains very similar to those resulted in the raw water sample, as represented by Figures $21 \mathrm{a}$ and $21 \mathrm{~b}$. Figure 23a, representing the high polymer concentration range in the filtered water sample, does show a slight gap between the maximum absorbance value at $190 \mathrm{~nm}$ of the $0.5 \mathrm{mg} / \mathrm{L}$ sample to the $1.0 \mathrm{mg} / \mathrm{L}$ sample. As this gap is not observed in Figure 23b, representing the low polymer concentration range in the same filtered water sample, this can be attributed to an error in polymer dosing during sample preparation. When comparing the maximum absorbance values between the filtered and raw samples at 190nm reported using the in-line unit, the filtered samples demonstrated a slight increase in absorbance to that of the raw water samples. This effect is presumed to be because of the lack of particulate matter in the sample available for adsorption causes the polymer to remain suspended in solution as residual. The increase in residual polymer would lead to an increase in the reported absorbance value, which is observed comparing Figures $21 \mathrm{a}$ and $21 \mathrm{~b}$ to Figures $23 \mathrm{a}$ and $23 \mathrm{~b}$. The fact that this effect is only observed using the in-line unit and not the desktop unit indicates that the in-line is capable of detecting polymer more sensitively, as it was able to measure this very slight increase in residual polymer concentration and demonstrate this through the absorbance spectra.

Another bulk sample of raw Rideau River water was then collected on another date, with a turbidity measuring 3.2NTU. Based on the absorbance reading for the high concentration range reporting a value between 2.0 A.U. and 2.5 A.U. using the desktop 
spectrophotometer, the sample was diluted 1:1 with deionized water prior to polymer addition in an attempt to bring the absorbance down to a value within the common acceptable working absorbance range of $0-1.0$ A.U. After dilution, the turbidity was decreased to a value of $1.2 \mathrm{NTU}$. Another purpose of the dilution was to observe the effect of suspended particulate matter present in the raw water sample on the sensitivity of UVvis spectroscopy as a method for the detection of residual polymer. As was done for the other datasets, the samples at both the high and low concentration ranges were analyzed using both the desktop and in-line UV-vis spectrophotometers. Figure 24 below represents the absorbance spectra for the $0-1 \mathrm{mg} / \mathrm{L}$ polymer concentration range using a $10 \mathrm{~mm}$ path length flow cell and the desktop spectrophotometer in the unfiltered and diluted Rideau River water sample. The diluted high concentration range samples were not measured using the desktop unit, as the 1:1 dilution is still out of the units working range.

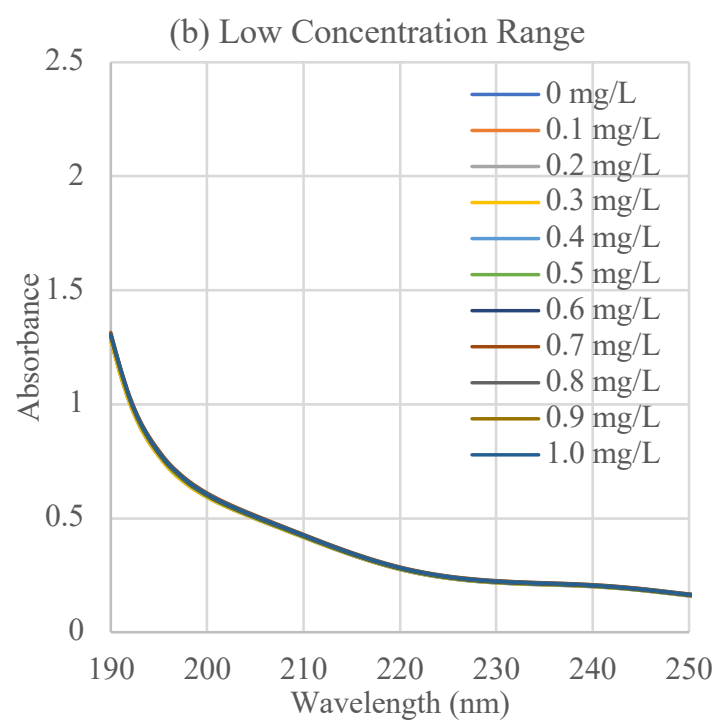

Figure 24: Absorbance spectra of Magnafloc LT27AG polymer in unfiltered Rideau River water diluted 1:1 with deionized water with an initial turbidity of 3.2NTU using a desktop spectrophotometer with a 10mm flow cell in the concentration range of $0-1 \mathrm{mg} / \mathrm{L}$ 
Figures $25 \mathrm{a}$ and $25 \mathrm{~b}$ below represent the absorbance spectra for the $0-3 \mathrm{mg} / \mathrm{L}$ and $0-$ $1 \mathrm{mg} / \mathrm{L}$ polymer concentration ranges respectfully using a $4 \mathrm{~mm}$ path length flow cell and the in-line spectrophotometer in the same unfiltered and diluted Rideau River water sample as the desktop unit above.

(a) High Concentration Range

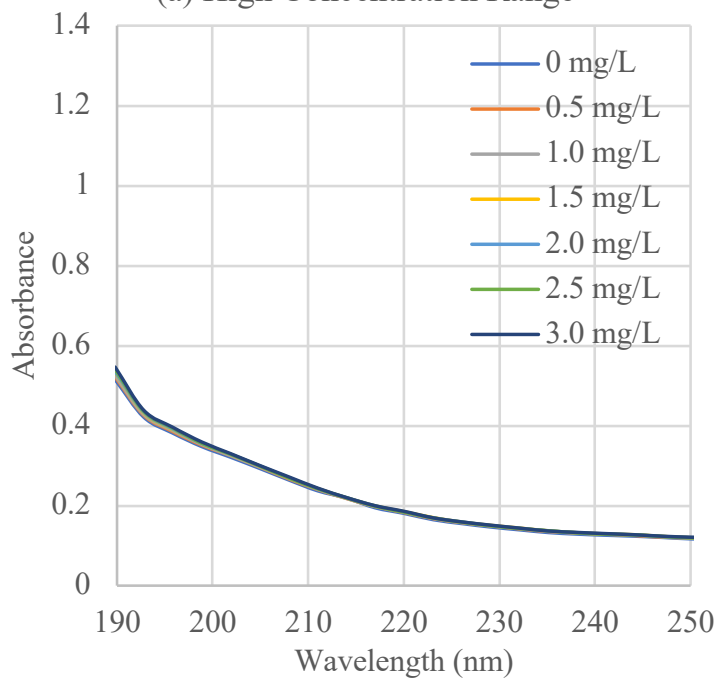

(b) Low Concentration Range

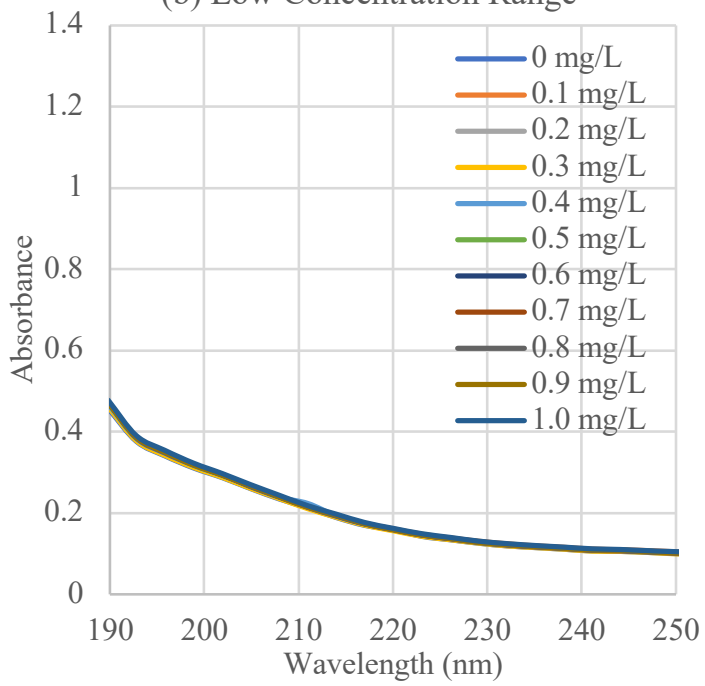

Figure 25: Absorbance spectra of Magnafloc LT27AG polymer in unfiltered Rideau River water diluted 1:1 with deionized water with an initial turbidity of 3.2NTU using an in-line spectrophotometer with a $4 \mathrm{~mm}$ flow cell in the concentration ranges of (a) $0-3 \mathrm{mg} / \mathrm{L}$ and (b) $0-1 \mathrm{mg} / \mathrm{L}$

When comparing the absorbance spectra of the diluted water samples, shown in Figures 24, 25a and 25b, to that of the undiluted water samples, shown in Figures 20a, 20b, 21a and 21b; a similar curve shape is produced. As was expected, the maximum absorbance values at $190 \mathrm{~nm}$ at both the high and low concentration ranges decreased to roughly half of what was demonstrated in the raw river water samples on both the desktop and the inline spectrophotometers. Aside from this decrease in maximum absorbance, no other major differences in the absorbance spectra can be drawn from the dilution of the samples. 
A portion of the bulk sample of raw Rideau River water measuring 3.2NTU turbidity was then filtered using a $0.45 \mu \mathrm{m}$ filter and also diluted $1: 1$ with deionized water prior to being dosed with Magnafloc LT27AG polymer at both the high and low concentration ranges. Again, this method was tested in order to observe the effects of suspended particulate matter present in the sample on the sensitivity of UV-vis spectroscopy for the detection of residual polymer. After dilution and filtration, the turbidity was decreased from 3.2NTU to $0.10 \mathrm{NTU}$. Again, both the high and low concentration ranges were analyzed using both the desktop and in-line UV-vis spectrophotometers. Figure 26 represents the absorbance spectra for the $0-1 \mathrm{mg} / \mathrm{L}$ polymer concentration range using the desktop spectrophotometer in the filtered and diluted Rideau River water sample. Again, the filtered high concentration range samples were not measured using the desktop unit as the 1:1 dilution still rendered absorbance values outside the units working range.

(b) Low Concentrartion Range

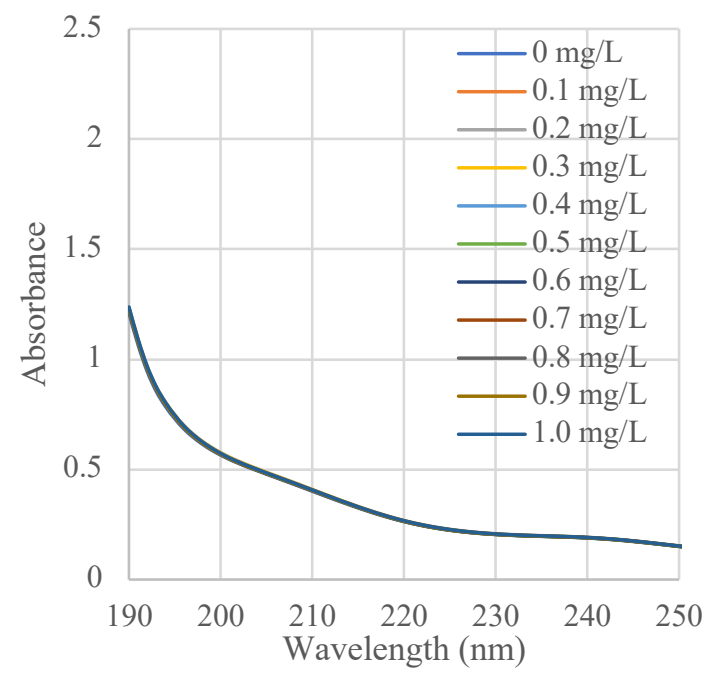

Figure 26: Absorbance spectra of Magnafloc LT27AG polymer in filtered Rideau River water filtered with a $0.45 \mu m$ filter and diluted 1:1 with deionized water with an initial turbidity of 3.2NTU using a desktop spectrophotometer with a $10 \mathrm{~mm}$ flow cell in the concentration range of $0-1 \mathrm{mg} / \mathrm{L}$ 
Figures $27 \mathrm{a}$ and $27 \mathrm{~b}$ below represent the absorbance spectra for the $0-3 \mathrm{mg} / \mathrm{L}$ and $0-1$ $\mathrm{mg} / \mathrm{L}$ polymer concentration ranges respectfully using a $4 \mathrm{~mm}$ path length flow cell and the in-line spectrophotometer in the same filtered and diluted Rideau River water sample as for the desktop unit above.

(a) High Concentration Range

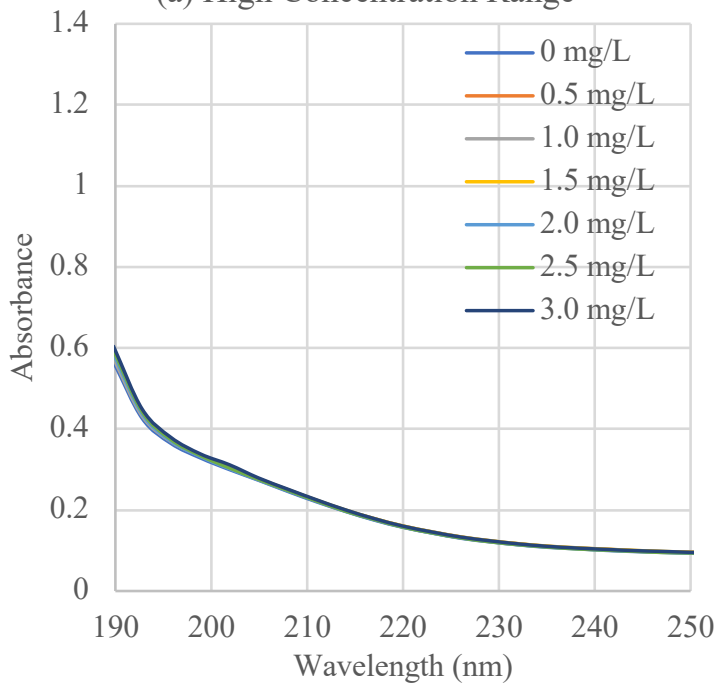

(b) Low Concentration Range

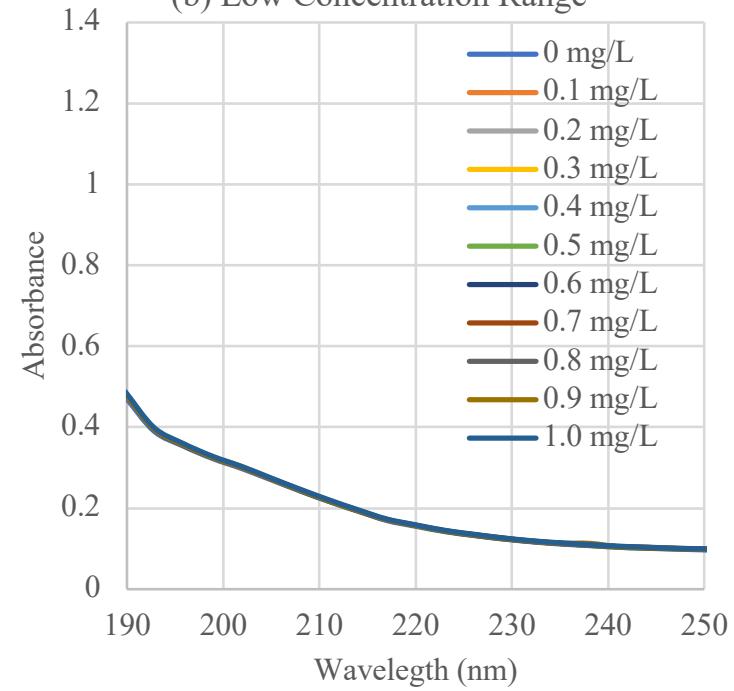

Figure 27: Absorbance spectra of Magnafloc LT27AG polymer in filtered Rideau River water filtered with a $0.45 \square m$ filter and diluted 1:1 with deionized water with an initial turbidity of 3.2NTU using an in-line spectrophotometer with a $4 \mathrm{~mm}$ flow cell in the concentration ranges of (a) $0-3 \mathrm{mg} / \mathrm{L}$ and (b) $0-1 \mathrm{mg} / \mathrm{L}$

When comparing the absorbance spectra of the filtered and diluted curves in Figures 26, $27 \mathrm{a}$ and $27 \mathrm{~b}$, to that of the raw river water absorbance spectra in Figures 20a, 20b, 21a and $21 \mathrm{~b}$; the shape is once again very similar. As was expected, the maximum absorbance values at $190 \mathrm{~nm}$ at both the high and low concentration ranges was decreased to the lowest value of all four samples using both the desktop and in-line spectrophotometers. The filtration and dilution of the samples remove the majority of the particulate matter present in the sample, and thus the absorbance attributed to this suspended particulate matter is no longer visible on the absorbance spectra. This causes the overall absorbance to decrease. 
Regardless of this, the incremental increases in polymer addition are still visible at 190nm in all cases, and the absorbance spectra curves retain their shape.

A full comparison of the peak absorbance values between the filtered samples, diluted samples, filtered and diluted samples, as well as the raw samples dosed with the high concentration range of polymer $(0-3 \mathrm{mg} / \mathrm{L})$ can be observed in Table 9 below. When analyzing the magnitude of the absorbance values at $190 \mathrm{~nm}$ for each concentration in this range, it is apparent that that raw water measures the highest absorbance values at this wavelength for each concentration the desktop spectrophotometer, however the in-line spectrophotometer reports the highest absorbance values in filtered water when comparing the raw, filtered and diluted samples. This same trend in absorbance is observed in the samples dosed with the low concentration range of polymer $(0-1 \mathrm{mg} / \mathrm{L})$, which are summarized in Table 10 below. The increase in peak absorbance between the raw and filtered samples can be attributed to the fact that when particles are absent in the sample, the polymer remains suspended in solution rather than adsorbed onto the particles. When polymer absorbance occurs, a portion of the polymer now attributed to the particle will form clumps which increase in weight and may settle out of suspension in solution. Seeing that peak absorbance can be correlated with polymer concentration, this effect would be indicated by a decrease in absorbance as the settled polymer would no longer be suspended in solution and would not be detected by the spectrophotometer. This is observed using the in-line spectrophotometer, showing a lower absorbance in the raw water when particles are present and a higher absorbance in the filtered water where particles are absent. This effect is observed on the in-line spectrophotometer and not the desktop spectrophotometer 
because, as was demonstrated in phase one of the results, the in-line spectrophotometer is capable of detecting residual polymer with more sensitivity than the desktop spectrophotometer. This indicates that this effect may not be visible on the desktop unit due to its lack of sensitivity. 


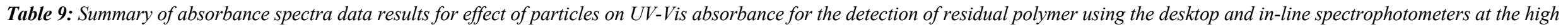
concentration range of Magnafloc LT27AG polymer $(0-3 \mathrm{mg} / \mathrm{L})$

\begin{tabular}{|c|c|c|c|c|c|c|c|c|c|c|c|c|}
\hline \multirow{3}{*}{ Concentration } & \multicolumn{4}{|c|}{ Raw Rideau River Sample } & \multicolumn{4}{|c|}{ Filtered Rideau River Sample } & \multirow{2}{*}{\multicolumn{2}{|c|}{$\begin{array}{c}\text { Diluted Rideau River } \\
\text { Sample } \\
\text { In-line }\end{array}$}} & \multirow{2}{*}{\multicolumn{2}{|c|}{$\begin{array}{c}\text { Filtered \& Diluted Rideau } \\
\text { River Sample } \\
\text { In-line }\end{array}$}} \\
\hline & \multirow[b]{2}{*}{ ABS } & & \multicolumn{2}{|c|}{ In-line } & \multicolumn{2}{|c|}{ Desktop } & \multicolumn{2}{|c|}{ In-line } & & & & \\
\hline & & $\begin{array}{c}\text { ABS } \\
\text { Increase }\end{array}$ & $\mathrm{ABS}$ & $\begin{array}{c}\text { ABS } \\
\text { Increase }\end{array}$ & $\mathrm{ABS}$ & $\begin{array}{c}\text { ABS } \\
\text { Increase }\end{array}$ & ABS & $\begin{array}{c}\text { ABS } \\
\text { Increase }\end{array}$ & ABS & $\begin{array}{c}\text { ABS } \\
\text { Increase }\end{array}$ & ABS & $\begin{array}{c}\text { ABS } \\
\text { Increase }\end{array}$ \\
\hline $\mathbf{0}$ & 2.2845 & & 1.0473 & & 2.1886 & & 0.9671 & & 0.5153 & & 0.5658 & \\
\hline 0.5 & 2.2933 & 0.0088 & 1.0565 & 0.0093 & 2.1694 & 0.0192 & 0.9759 & 0.0088 & 0.5221 & 0.0067 & 0.5761 & 0.0103 \\
\hline 1.0 & 2.3082 & 0.0149 & 1.0645 & 0.0080 & 2.1473 & 0.0221 & 1.1271 & 0.1512 & 0.5258 & 0.0037 & 0.5745 & 0.0015 \\
\hline 1.5 & 2.3186 & 0.0104 & 1.0750 & 0.0104 & 2.1758 & 0.0284 & 1.1632 & 0.0361 & 0.5336 & 0.0079 & 0.5860 & 0.0115 \\
\hline 2.0 & 2.3315 & 0.0129 & 1.0848 & 0.0099 & 2.1988 & 0.0231 & 1.1786 & 0.0153 & 0.5353 & 0.0017 & 0.5850 & 0.0011 \\
\hline 2.5 & 2.3420 & 0.0105 & 1.0936 & 0.0088 & 2.2060 & 0.0072 & 1.1918 & 0.0132 & 0.5403 & 0.0050 & 0.5942 & 0.0093 \\
\hline 3.0 & 2.3568 & 0.0148 & 1.1019 & 0.0082 & 2.2324 & 0.0264 & 1.2087 & 0.0169 & 0.5464 & 0.0061 & 0.6042 & 0.0100 \\
\hline $\begin{array}{c}\text { Average } \\
\text { Incremental } \\
\text { Increase }\end{array}$ & & 0.0121 & & 0.0091 & & 0.0073 & & 0.0403 & & 0.0052 & & 0.0064 \\
\hline $\begin{array}{c}\text { STDV of } \\
\text { Incremental } \\
\text { Increase }\end{array}$ & & 0.0025 & & 0.0009 & & 0.0229 & & 0.0551 & & 0.0022 & & 0.0060 \\
\hline
\end{tabular}




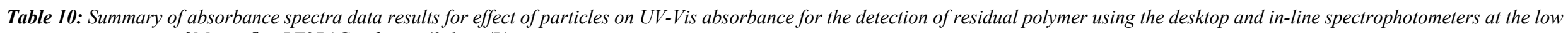
concentration range of Magnafloc LT27AG polymer $(0-1 \mathrm{mg} / \mathrm{L})$

\begin{tabular}{|c|c|c|c|c|c|c|c|c|c|c|c|c|c|c|c|c|}
\hline \multirow{3}{*}{ Concentration } & \multicolumn{4}{|c|}{ Raw Rideau River Sample } & \multicolumn{4}{|c|}{ Filtered Rideau River Sample } & \multicolumn{4}{|c|}{ Diluted Rideau River Sample } & \multicolumn{4}{|c|}{$\begin{array}{c}\text { Filtered \& Diluted Rideau River } \\
\text { Sample }\end{array}$} \\
\hline & \multicolumn{2}{|c|}{ Desktop } & \multicolumn{2}{|c|}{ In-line } & \multicolumn{2}{|c|}{ Desktop } & \multicolumn{2}{|c|}{ In-line } & \multicolumn{2}{|c|}{ Desktop } & \multicolumn{2}{|c|}{ In-line } & \multicolumn{2}{|c|}{ Desktop } & \multicolumn{2}{|c|}{ In-line } \\
\hline & ABS & $\begin{array}{c}\text { ABS } \\
\text { Increase }\end{array}$ & ABS & $\begin{array}{c}\text { ABS } \\
\text { Increase }\end{array}$ & ABS & $\begin{array}{c}\text { ABS } \\
\text { Increase }\end{array}$ & ABS & $\begin{array}{c}\text { ABS } \\
\text { Increase }\end{array}$ & ABS & $\begin{array}{c}\text { ABS } \\
\text { Increase }\end{array}$ & ABS & $\begin{array}{c}\text { ABS } \\
\text { Increase }\end{array}$ & ABS & $\begin{array}{c}\text { ABS } \\
\text { Increase }\end{array}$ & ABS & $\begin{array}{c}\text { ABS } \\
\text { Increase }\end{array}$ \\
\hline $\mathbf{0}$ & 1.9261 & & 0.8981 & & 1.9616 & & 1.0326 & & 1.2809 & & 0.4582 & & 1.2094 & & 0.4756 & \\
\hline 0.2 & 1.9328 & 0.0258 & 0.9173 & 0.0050 & 1.9631 & 0.0029 & 1.0460 & 0.0008 & 1.2815 & 0.0047 & 0.4626 & 0.0037 & 1.2369 & 0.0071 & 0.4858 & 0.0043 \\
\hline 0.3 & 1.9600 & 0.0272 & 0.9219 & 0.0046 & 1.9613 & 0.0018 & 1.0491 & 0.0032 & 1.2801 & 0.0014 & 0.4626 & 0.0000 & 1.2335 & 0.0034 & 0.4854 & 0.0004 \\
\hline 0.4 & 1.9785 & 0.0185 & 0.9282 & 0.0063 & 1.9597 & 0.0016 & 1.0480 & 0.0011 & 1.3076 & 0.0275 & 0.4684 & 0.0058 & 1.2237 & 0.0098 & 0.4828 & 0.0026 \\
\hline 0.5 & 1.9637 & 0.0148 & 0.9320 & 0.0038 & 1.9623 & 0.0025 & 1.0534 & 0.0054 & 1.2972 & 0.0104 & 0.4710 & 0.0026 & 1.2273 & 0.0036 & 0.4839 & 0.0011 \\
\hline 0.6 & 1.9891 & 0.0255 & 0.9380 & 0.0060 & 1.9655 & 0.0032 & 1.0519 & 0.0015 & 1.2966 & 0.0006 & 0.4732 & 0.0022 & 1.2222 & 0.0052 & 0.4829 & 0.0010 \\
\hline 0.8 & 2.0129 & 0.0183 & 0.9461 & 0.0031 & 1.9627 & 0.0052 & 1.0544 & 0.0013 & 1.3052 & 0.0095 & 0.4729 & 0.0019 & 1.2231 & 0.0006 & 0.4836 & 0.0004 \\
\hline 0.9 & 2.0276 & 0.0147 & 0.9512 & 0.0051 & 1.9748 & 0.0121 & 1.0576 & 0.0032 & 1.3025 & 0.0028 & 0.4719 & 0.0010 & 1.2254 & 0.0023 & 0.4834 & 0.0002 \\
\hline 1.0 & 2.0232 & 0.0044 & 0.9546 & 0.0034 & 1.9724 & 0.0024 & 1.0636 & 0.0060 & 1.3059 & 0.0035 & 0.4781 & 0.0062 & 1.2376 & 0.0122 & 0.4886 & 0.0052 \\
\hline $\begin{array}{c}\text { Average } \\
\text { Incremental } \\
\text { Increase }\end{array}$ & & 0.0097 & & 0.0056 & & 0.0011 & & 0.0031 & & 0.0025 & & 0.0020 & & 0.0028 & & 0.0013 \\
\hline $\begin{array}{c}\text { STDV of } \\
\text { Incremental } \\
\text { Increase }\end{array}$ & & 0.0192 & & 0.0032 & & 0.0048 & & 0.0041 & & 0.0120 & & 0.0038 & & 0.0088 & & 0.0029 \\
\hline
\end{tabular}




\subsubsection{Linear correlations}

The results from Figures 20 through 27 above demonstrate that the absorbance values at a 190nm wavelength were highest for all samples on both the in-line spectrophotometer and the desktop spectrophotometer. These results remained valid regardless of dilution, filtration, or a combination of the two; indicating that suspended particulates present in the sample do not alter the wavelength at which the polymer is most highly detected by UVvis spectroscopy. Figures 20 through 27 also demonstrate that the absorbance values maintain their incremental increase as polymer concentration is increased, as shown phase one of the results. In order to further analyze this relationship and determine the impact that suspended particulates present in raw water may have on UV-vis spectroscopy sensitivity for polymer detection, a linear correlation of the absorbance-polymer concentration data was done for all datasets.

Figures $28 \mathrm{a}$ and $28 \mathrm{~b}$ below represent the positive linear correlation between the polymer concentration and absorbance values at $190 \mathrm{~nm}$ using the desktop spectrophotometer at the 0-3 $\mathrm{mg} / \mathrm{L}$ and the $0-1 \mathrm{mg} / \mathrm{L}$ concentration ranges in an unfiltered and undiluted Rideau River water sample respectively. Figures $29 \mathrm{a}$ and $29 \mathrm{~b}$ below represent the linear correlation for the $0-3 \mathrm{mg} / \mathrm{L}$ and $0-1 \mathrm{mg} / \mathrm{L}$ polymer concentration ranges respectfully using a $4 \mathrm{~mm}$ path length flow cell and the in-line spectrophotometer in the same unfiltered and undiluted Rideau River water sample as for the desktop unit. 
(a) High Concentration Range

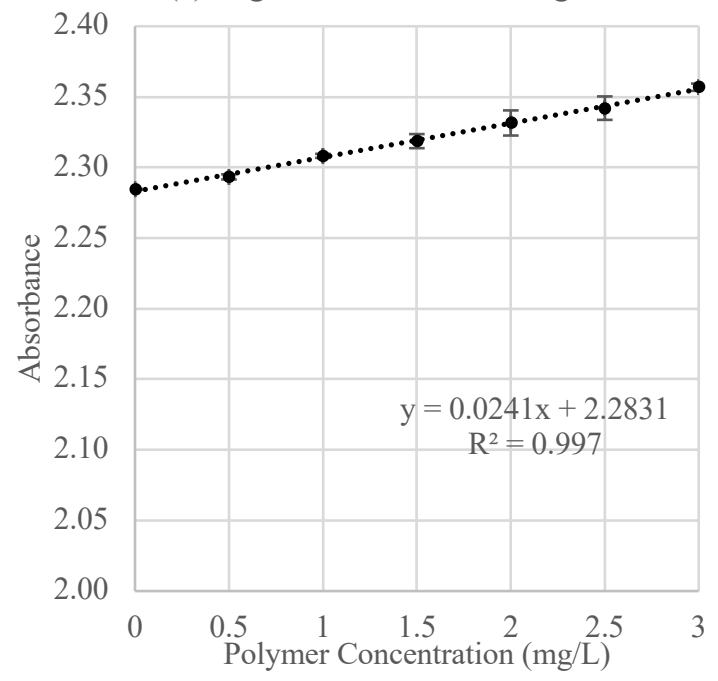

(b) Low Concentration Range

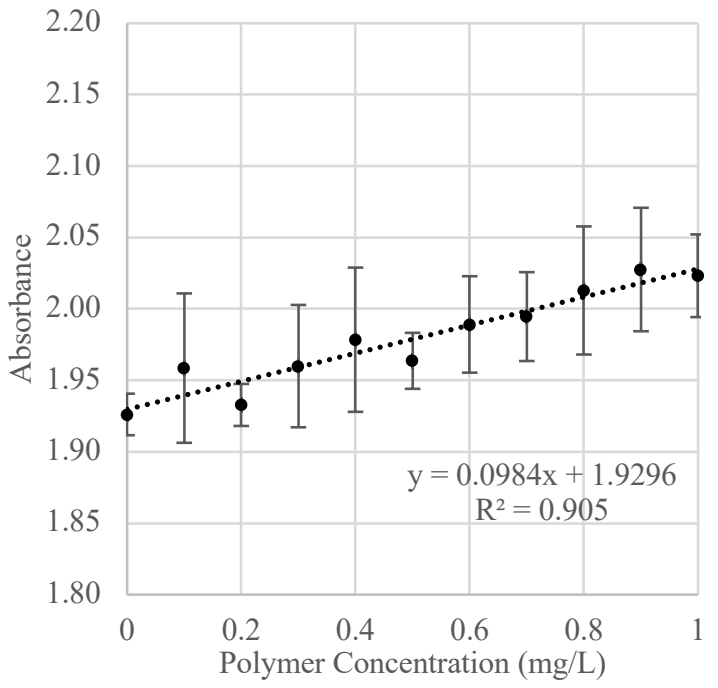

Figure 28: Linear correlation of the absorbance-polymer concentration data of Magnafloc LT27AG in Rideau River water with a turbidity of $6.8 N T U$ at $190 \mathrm{~nm}$ using a desktop spectrophotometer with a $10 \mathrm{~mm}$ flow cell in the concentration ranges of (a) $0-3 \mathrm{mg} / \mathrm{L}$ and (b) $0-1 \mathrm{mg} / \mathrm{L}$

(a) High Concentration Range

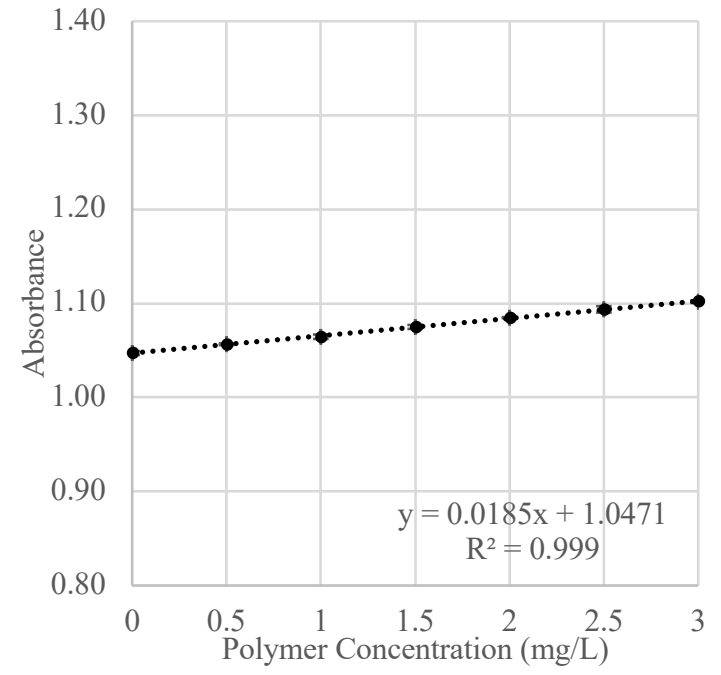

(b) Low Concentration Range

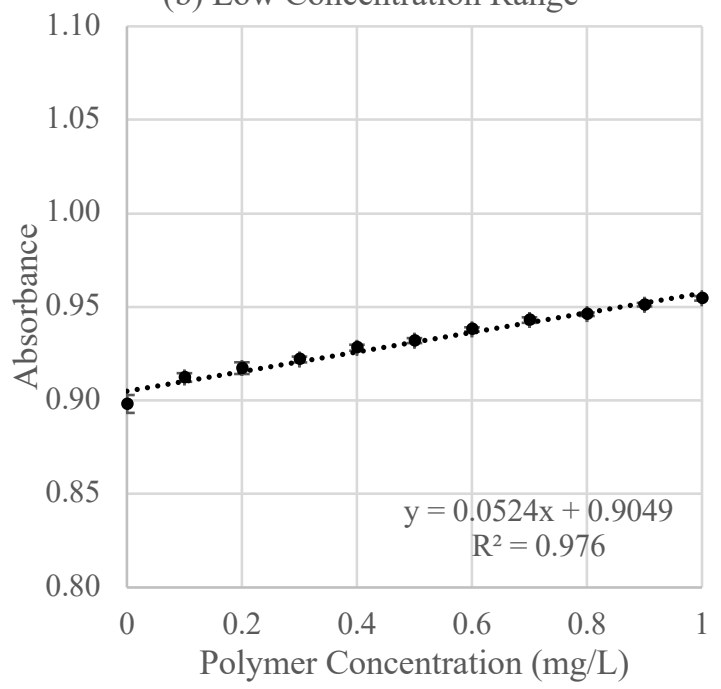

Figure 29: Linear correlation of the absorbance-polymer concentration data of Magnafloc LT27AG in Rideau River water with a turbidity of 6.8NTU at 190nm using an in-line spectrophotometer with a 4mm flow cell in the concentration ranges of (a) $0-3 m g / L$ and (b) $0-1 m g / L$

From these linear correlations, it is apparent that the presence of particles in the water sample did not hinder the ability of UV-Vis spectroscopy to detect the increase in polymer 
concentration between the samples at both the high and low concentration ranges. Figures $28 \mathrm{a}$ and $28 \mathrm{~b}$, representing the absorbance values reported by the desktop unit, do correlate with a larger slope than that of the in-line unit measuring the same samples, represented by Figures 29a and 29b. The larger slope is an initial indication that the linear correlation between polymer concentration and absorbance may be detected more strongly using the desktop spectrophotometer, however there are other factors to be considered. Another notable difference between the two spectrophotometers is observed when comparing the linear correlations at the low concentration range. The error bars at each concentration in Figure 28b, representing the desktop unit absorbance values at this range, indicates that there is quite a large variance in absorbance readings for a given concentration. This variance is nowhere near as large for the same samples read using the in-line spectrophotometer, represented by Figure 29b. The large variance implies that the desktop unit has a larger variability for polymer detection at lower concentrations when compared to the in-line unit, which is quite meaningful as drinking water treatment applications require the detection of polymer at significantly low concentrations $(>1.0 \mathrm{mg} / \mathrm{L})$. The coefficient of determination is also stronger using the in-line unit $\left(\mathrm{R}^{2}=0.976\right)$ at the low concentration range when compared to the desktop unit $\left(\mathrm{R}^{2}=0.905\right)$.

Figures $30 \mathrm{a}$ and $30 \mathrm{~b}$ below represent the linear correlation for the $0-3 \mathrm{mg} / \mathrm{L}$ and $0-1 \mathrm{mg} / \mathrm{L}$ polymer concentration ranges respectfully using the desktop spectrophotometer in an undiluted Rideau River water sample filtered through a $0.45 \mu \mathrm{m}$ filter. Figures $31 \mathrm{a}$ and $31 \mathrm{~b}$ below represent the linear correlations for the $0-3 \mathrm{mg} / \mathrm{L}$ and $0-1 \mathrm{mg} / \mathrm{L}$ polymer 
concentration ranges, respectively, using the in-line spectrophotometer under the same sample conditions as for the desktop spectrophotometer.

(a) High Concentration Range

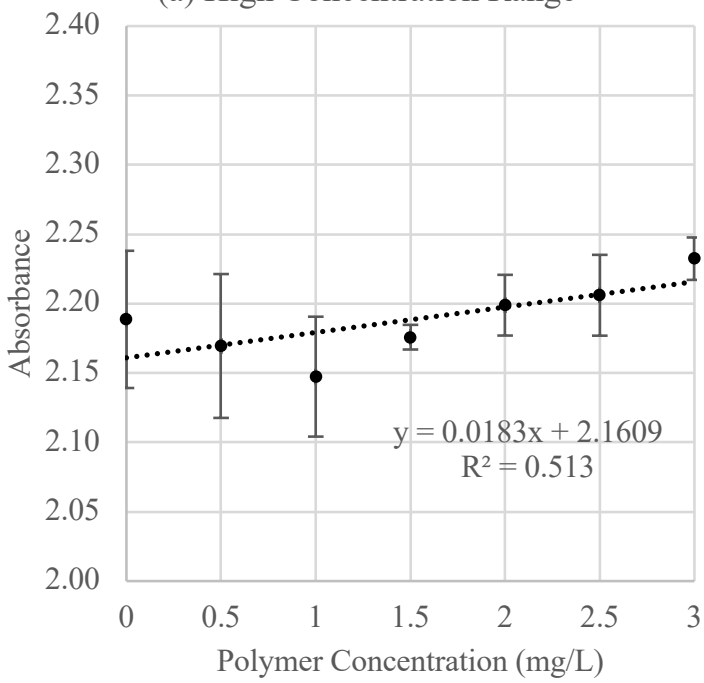

(b) Low Concentration Range

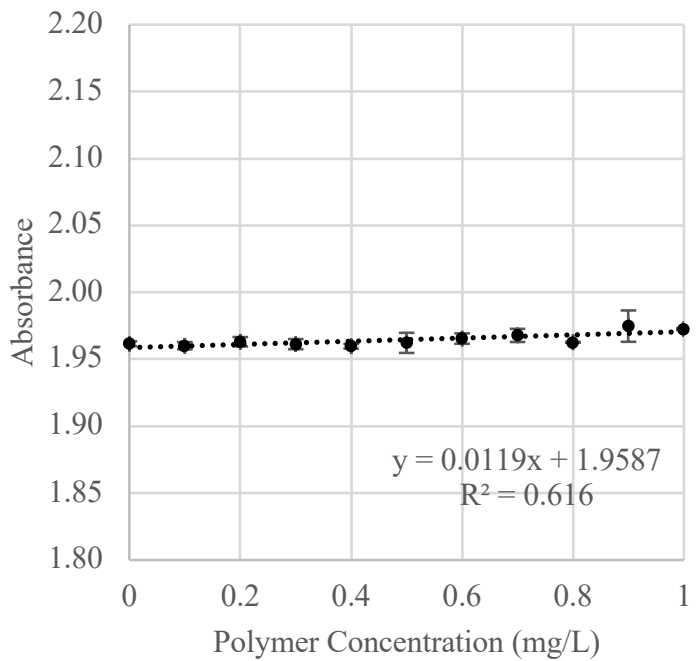

Figure 30: Linear correlation of Magnafloc LT27AG polymer in filtered (0.45 $\mu \mathrm{m})$ and undiluted Rideau River water with an initial turbidity of $6.8 N T U$ using a desktop spectrophotometer with a $10 \mathrm{~mm}$ flow cell in the concentration ranges of (a) $0-3 \mathrm{mg} / \mathrm{L}$ and (b) $0-1 \mathrm{mg} / \mathrm{L}$

(a) High Concentration Range

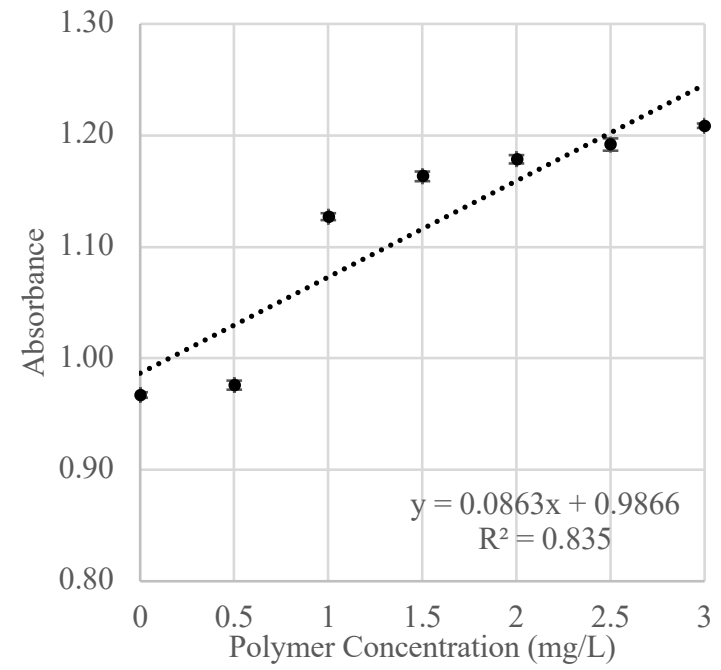

(b) Low Concentration Range

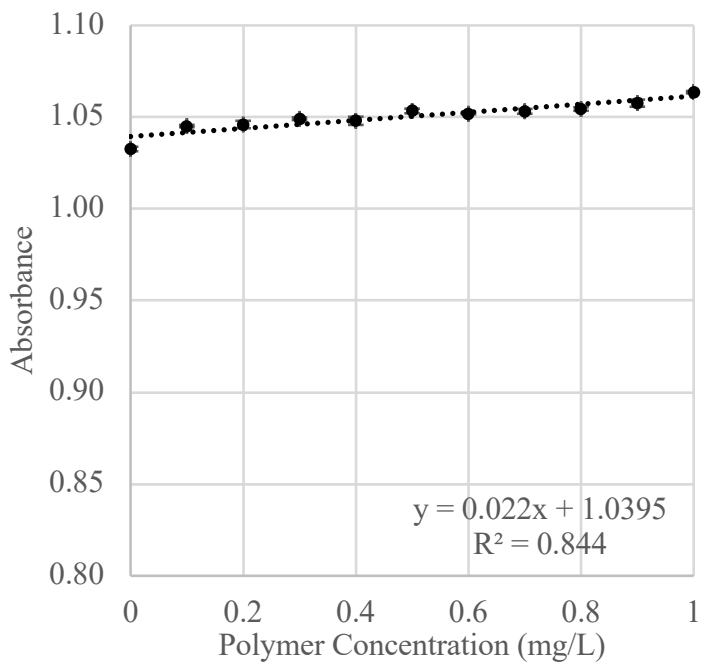

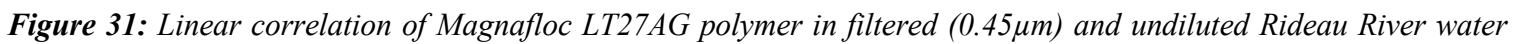
with an initial turbidity of $6.8 N T U$ using an in-line spectrophotometer with a $4 \mathrm{~mm}$ flow cell in the concentration ranges of (a) $0-3 \mathrm{mg} / \mathrm{L}$ and (b) $0-1 \mathrm{mg} / \mathrm{L}$ 
The filtration and removal of particles from the sample decreased the linear correlation between absorbance and polymer concentration at both the high and low concentration ranges on both spectrophotometers. The linear correlations were weaker using the desktop unit when compared to the in-line unit, indicated by the lower coefficient of determinations reported in Figures 30a and 30b when compared to those reported by Figures 31a and 31b. The slope of the linear correlations at the low concentration range was also lower on both spectrophotometers when sample filtration was implemented when compared to that of the raw water sample in Figures $28 \mathrm{~b}$ and $29 \mathrm{~b}$. These results can be correlated with the differences in absorbance spectra between the raw water, in Figures 20 and 21, and the filtered water, in Figures 22 and 23. As was mentioned, the lack of particles in the water causes most of the polymer to remain suspended in solution as residual, contributing to an increase in maximum absorbance. However, the filtration may not have removed $100 \%$ of the particles present in the water, causing a discrepancy in sample uniformity. This would lead to the potential of some samples of a given polymer concentration containing more particles than another given polymer concentration. This discrepancy would cause certain samples to experience polymer settling more so than others, thus leading to the observed decrease in linear correlation. Despite the decrease, the in-line unit was still able to form quite a strong liner correlation between polymer concentration and absorbance at 190nm in in the filtered water samples, at both the high polymer concentration range $\left(\mathrm{R}^{2}=0.835\right)$ and the low polymer concentration range $\left(\mathrm{R}^{2}=0.844\right)$.

Figure 32 below represents the linear correlation for the $0-1 \mathrm{mg} / \mathrm{L}$ polymer concentration range using a $10 \mathrm{~mm}$ path length flow cell and the desktop spectrophotometer in an 
unfiltered Rideau River water sample diluted 1:1 with deionized water. Figures 33a and $33 \mathrm{~b}$ represent the linear correlations for the $0-3 \mathrm{mg} / \mathrm{L}$ and $0-1 \mathrm{mg} / \mathrm{L}$ polymer concentration ranges, respectively, using the in-line spectrophotometer under the same sample conditions as for the desktop spectrophotometer.

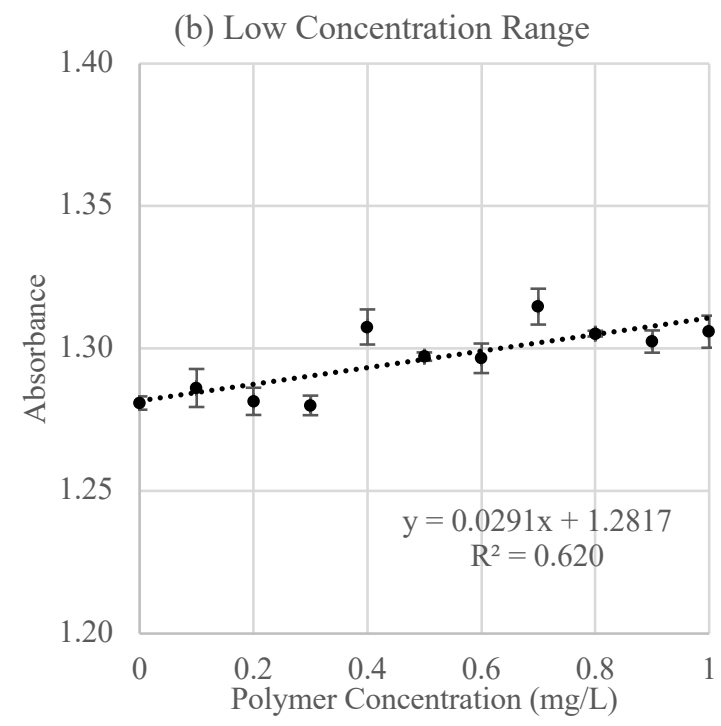

Figure 32: Linear correlation of Magnafloc LT27AG polymer in unfiltered Rideau River water diluted 1:1 with deionized water with an initial turbidity of $6.8 N T U$ using a desktop spectrophotometer with a $10 \mathrm{~mm}$ flow cell in the concentration range of $0-1 \mathrm{mg} / \mathrm{L}$

(a) High Concentration Range

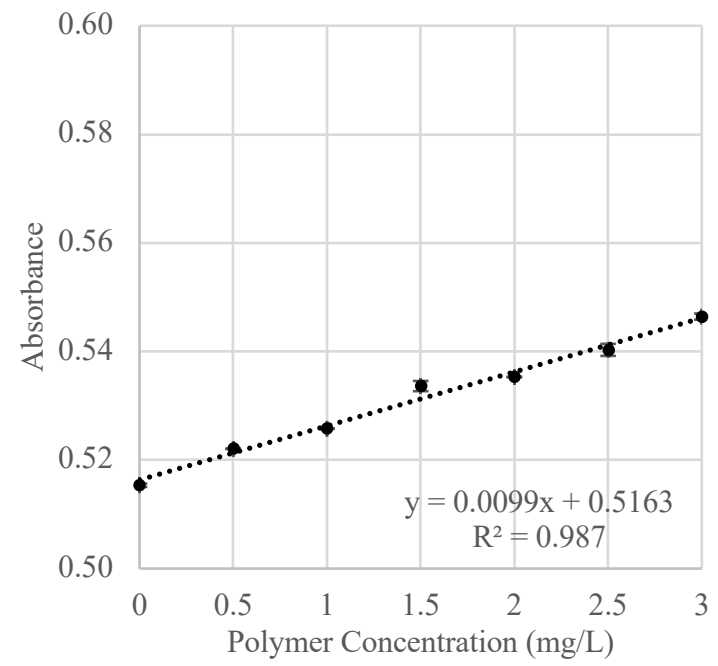

(b) Low Concentration Range

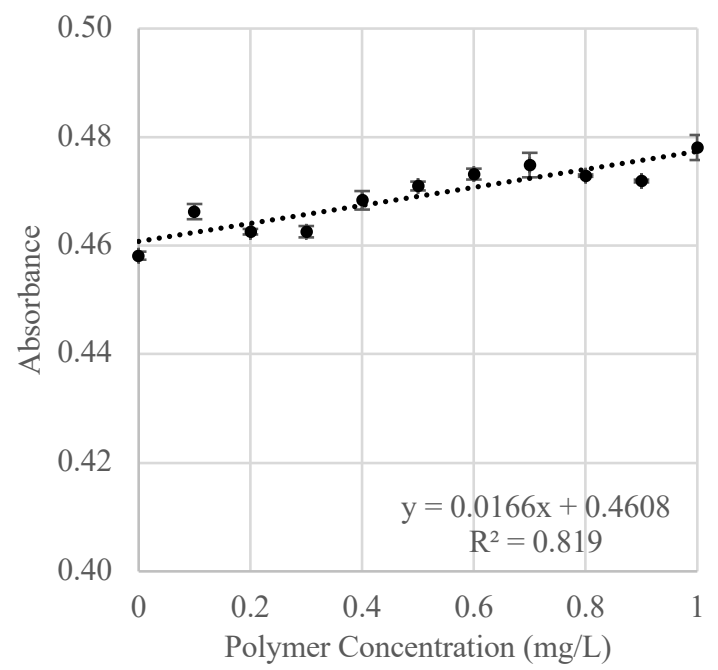

Figure 33: Linear correlation of Magnafloc LT27AG polymer in unfiltered Rideau River water diluted 1:1 with deionized water with an initial turbidity of 6.8NTU using an in-line spectrophotometer with a $4 \mathrm{~mm}$ flow cell in the concentration ranges of (a) $0-3 \mathrm{mg} / \mathrm{L}$ and (b) $0-1 \mathrm{mg} / \mathrm{L}$ 
As expected, the 1:1 dilution of the sample with deionized water decreased the maximum absorbance of each sample to half of its value reported in the raw water samples. This may be necessary if the raw water absorbance values reported are outside the working range of the spectrophotometer which is $0-4$ A.U., however the suggested range is $0-1$ A.U. Dilution of the sample decreased the linear correlation between peak absorbance and polymer concentration quite significantly using the desktop spectrophotometer, as observed in Figure $32 \mathrm{~b}\left(\mathrm{R}^{2}=0.620\right)$ when compared to the raw water sample in Figure $28 \mathrm{~b}$ $\left(\mathrm{R}^{2}=0.925\right)$. The in-line spectrophotometer, however, was still able to form quite a strong linear correlation post dilution at both the high and low concentration ranges, as observed by the linear correlation coefficients in Figure $33 \mathrm{a}\left(\mathrm{R}^{2}=0.987\right)$ and Figure $33 \mathrm{~b}\left(\mathrm{R}^{2}=\right.$ $0.819)$.

Figure 34 below represents the linear correlation for the $0-1 \mathrm{mg} / \mathrm{L}$ polymer concentration range using the desktop spectrophotometer in a Rideau River water sample both filtered through a 0.45 filter and diluted 1:1 with deionized water. Figures $35 \mathrm{a}$ and $35 \mathrm{~b}$ represent the linear correlations for the $0-3 \mathrm{mg} / \mathrm{L}$ and $0-1 \mathrm{mg} / \mathrm{L}$ polymer concentration ranges, respectively, using the in-line spectrophotometer under the same sample conditions as that of the desktop unit. 
(b) Low Concentration Range

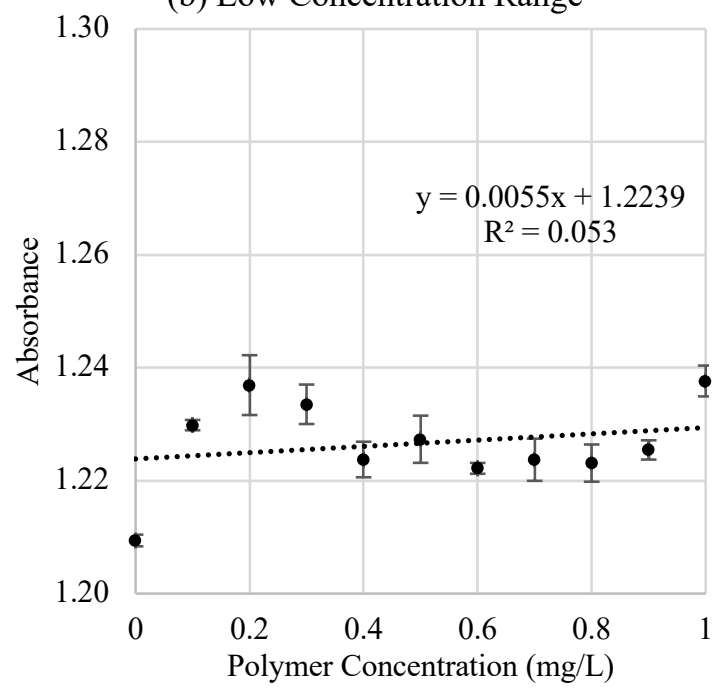

Figure 34: Linear correlation of Magnafloc LT27AG polymer in filtered $(0.45 \mu \mathrm{m})$ Rideau River water diluted 1:1 with deionized water with an initial turbidity of 3.2NTU using a desktop spectrophotometer with a 10mm flow cell in the concentration range of $0-1 \mathrm{mg} / \mathrm{L}$

(a) High Concentration Range

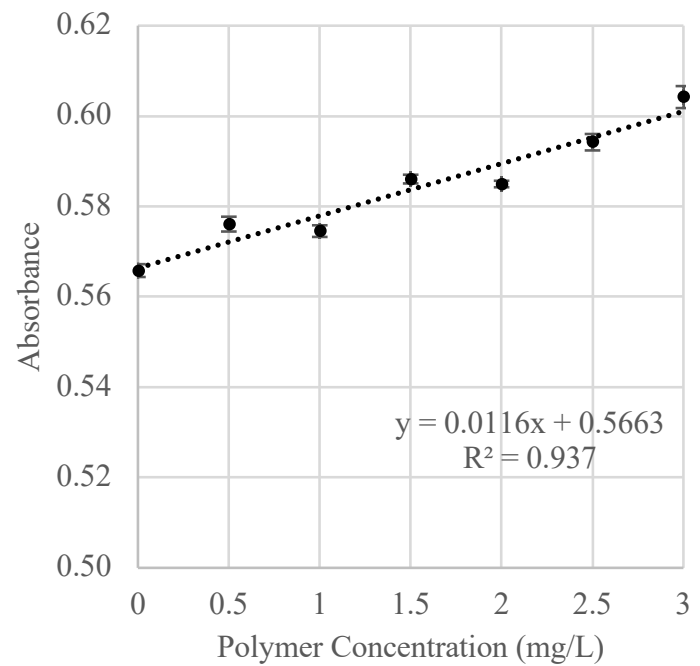

(b) Low Concentration Range

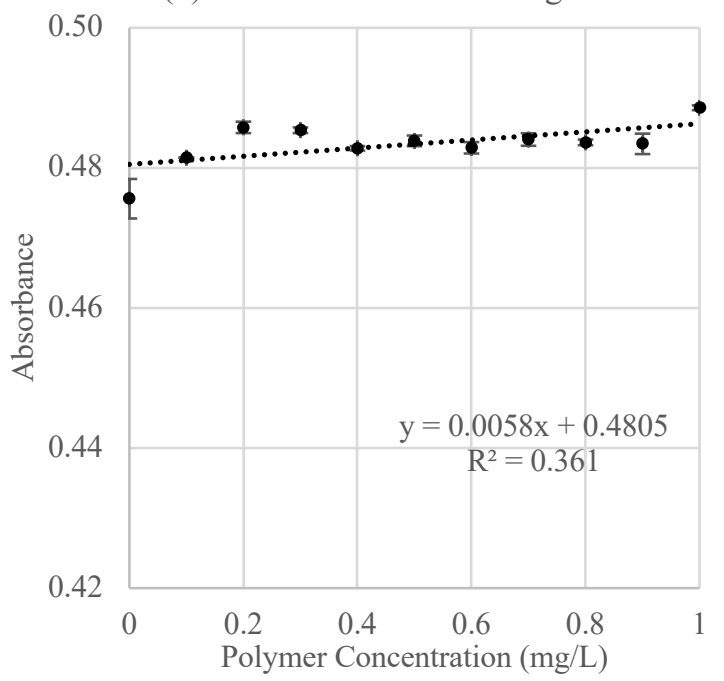

Figure 35: Linear correlation of Magnafloc LT27AG polymer in filtered (0.45 $4 \mathrm{~m})$ Rideau River water diluted 1:1 with deionized water with an initial turbidity of 3.2NTU using an in-line spectrophotometer with a $4 \mathrm{~mm}$ flow cell in the concentration ranges of (a) 0-3 $\mathrm{mg} / \mathrm{L}$ and (b) $0-1 \mathrm{mg} / \mathrm{L}$

As was expected, a 1:1 dilution of the samples with deionized water once again decreased the peak absorbance values to half their value reported in the raw water samples at both the high and low concentration ranges on both spectrophotometers. The addition of the filtration of the samples also decreased the linear correlation between the absorbance at 
$190 \mathrm{~nm}$ and polymer concentration in all cases. The correlation was completely lost at the low concentration range using the desktop spectrophotometer, as observed in Figure $34\left(\mathrm{R}^{2}\right.$ $=0.053)$. The in-line spectrophotometer was still able to detect quite a strong linear correlation at the high concentration range, visible in Figure $35 \mathrm{a}(\mathrm{R}=0.936)$, however at the low concentration range the correlation also decreased substantially, visible in Figure $35 \mathrm{~b}\left(\mathrm{R}^{2}=0.361\right)$. Tables 11 and 12 below summarize the coefficient of determination values for all samples at both the high and low polymer concentration ranges, respectively.

Table 11: Summary of coefficient of determinations for desktop and in-line UV-vis spectrophotometers at a high Magnafloc LT27AG polymer concentration range $(0-3 \mathrm{mg} / \mathrm{L})$ in Rideau River water samples

\begin{tabular}{lccc}
\hline \multicolumn{1}{c}{$\begin{array}{c}\text { UV-vis spectrophotometer } \\
\text { model }\end{array}$} & Filtered & Diluted & $\begin{array}{c}\text { Coefficient of } \\
\text { determination } \mathbf{R}^{\mathbf{2}}\end{array}$ \\
\hline Desktop Unit & No & No & 0.997 \\
\hline In-Line Unit & No & No & 0.999 \\
\hline Desktop Unit & Yes & No & 0.513 \\
\hline In-Line Unit & Yes & No & 0.835 \\
\hline In-Line Unit & No & Yes & 0.987 \\
\hline In-Line Unit & Yes & Yes & 0.936 \\
\hline
\end{tabular}

Table 12: Summary of coefficient of determinations for desktop and in-line UV-vis spectrophotometers at a low Magnafloc LT27AG polymer concentration range (0-1 mg/L) in Rideau River water samples

\begin{tabular}{lccc}
\multicolumn{1}{c}{ UV-vis spectrophotometer } & Filtered & Diluted & $\begin{array}{c}\text { Coefficient of } \\
\text { determination } \mathbf{R}^{\mathbf{2}}\end{array}$ \\
\hline Desktop Unit & No & No & 0.905 \\
\hline In-Line Unit & No & No & 0.976 \\
\hline Desktop Unit & Yes & No & 0.616 \\
\hline In-Line Unit & Yes & No & 0.844 \\
\hline Desktop Unit & No & Yes & 0.620 \\
\hline In-Line Unit & No & Yes & 0.819 \\
\hline Desktop Unit & Yes & Yes & 0.053 \\
\hline In-Line Unit & Yes & Yes & 0.361 \\
\hline
\end{tabular}




\subsection{Effect of water treatment process chemicals on polymer detection using $U V$-vis spectroscopy}

\subsubsection{UV-vis absorbance spectra}

In order to assess the feasibility of UV-vis spectroscopy as a method of determining the residual polymer concentration during the drinking water treatment process, the impact of additional chemicals that may potentially be present during treatment on the sensitivity of UV-vis spectroscopy as a method for this purpose were examined. The Actiflo ${ }^{\circledR}$ enhanced coagulation process, manufactured by Veolia Water Technologies Inc. (Paris, France), employed at the Casselman drinking water treatment facility was chosen to be recreated at a laboratory scale for the purpose of this experiment, as it utilizes a variety of additional chemicals during its process. Firstly, before clarification, potassium permanganate is added to the raw water to oxidize any iron and/or manganese present. Following this, a polyaluminium silicate sulphate (PASS-10) coagulant manufactured by Kemira Inc. (Helsinki, Finland) is added to destabilize colloidal material as the water enters the clarifier. The water is then sent to an injection tank where the polymer, used as a flocculant in this case, is added along with microsand to aid in settling through the creation of larger, heavier flocs. The water is then mixed and sent to a decantation tank and left to settle for thirty minutes. Following this, the supernatant is sent to a Dunsenflo ${ }^{\circledR}$ filter, also manufactured by Veolia Water Technologies Inc., which is composed of anthracite and sand. Postfiltration, sodium hydroxide is added as needed to ensure the water maintains a neutral $\mathrm{pH}$. 
These chemicals were dosed into samples of both deionized water and Rideau River water samples at dosages in accordance with those reported by the Casselman Water Treatment facility. A full list of these dosage concentrations is reported in Table 4 found in Chapter 3: Materials and Methods, as well as a full description of sample preparation methodology. The dosage of potassium permanganate, PASS-10 coagulant, and microsand remained constant for all samples. The polymer dosages, however, varied between a high concentration range $(0-3 \mathrm{mg} / \mathrm{L})$ and a low concentration range $(0-1 \mathrm{mg} / \mathrm{L})$. The amount of sodium hydroxide also varied between samples and was dosed as needed in order to acquire a $\mathrm{pH}$ of 7 for each sample. Post mixing and settling, the supernatant of each sample was collected and used for absorbance measurements on both the desktop and in-line spectrophotometers. Figures $36 \mathrm{a}$ and $36 \mathrm{~b}$ below represent the absorbance spectra for sample supernatant in the $0-3 \mathrm{mg} / \mathrm{L}$ and $0-1 \mathrm{mg} / \mathrm{L}$ polymer concentration ranges, respectfully, using the desktop spectrophotometer in deionized water samples dosed with all Actiflo® process chemicals.

(a) High Concentration Range

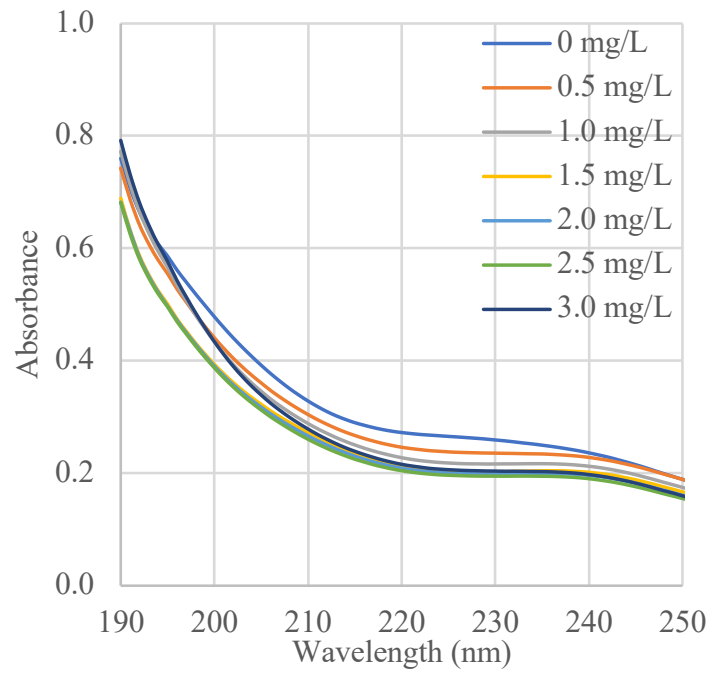

(b) Low Concentration Range

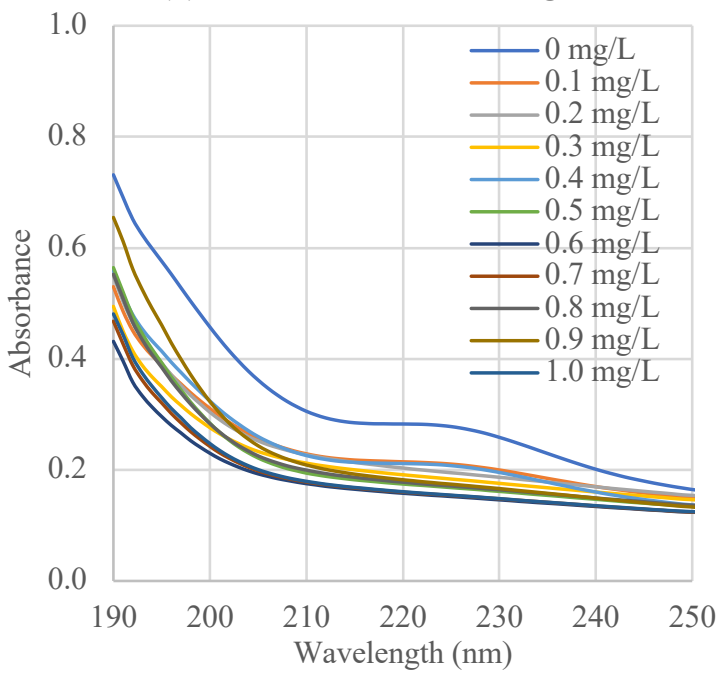

Figure 36: Absorbance spectra of Magnafloc LT27AG polymer in deionized water dosed with Actiflo $®$ enhanced coagulation process chemicals using a desktop spectrophotometer with a $10 \mathrm{~mm}$ flow cell in the concentration ranges of (a) $0-3 \mathrm{mg} / \mathrm{L}$ and (b) $0-1 \mathrm{mg} / \mathrm{L}$ 
Figures $37 \mathrm{a}$ and $37 \mathrm{~b}$ below represent the absorbance spectra for sample supernatant in the $0-3 \mathrm{mg} / \mathrm{L}$ and $0-1 \mathrm{mg} / \mathrm{L}$ polymer concentration ranges, respectfully, using the in-line spectrophotometer in the same deionized water samples as above dosed with all Actiflo ${ }^{\circledR}$ process chemicals.

(a) High Concentration Range

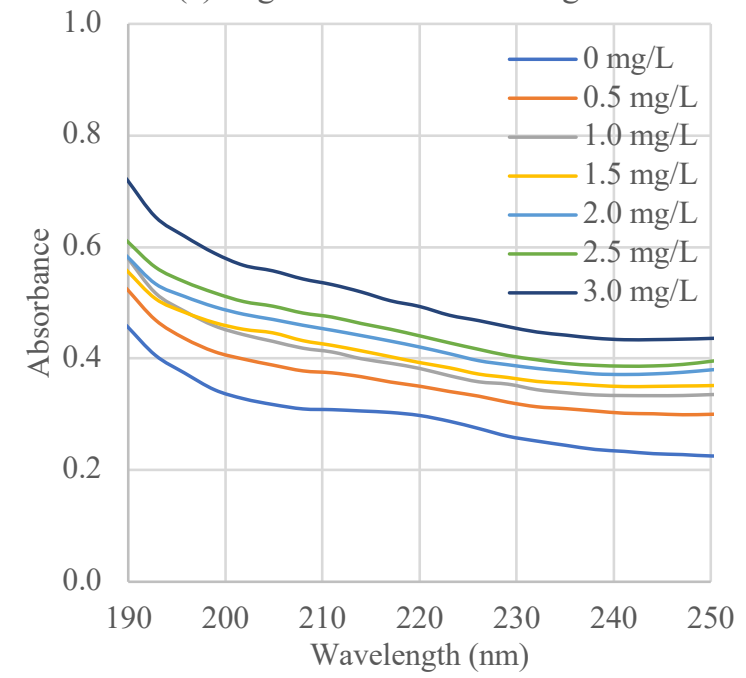

(b) Low Concentration Range

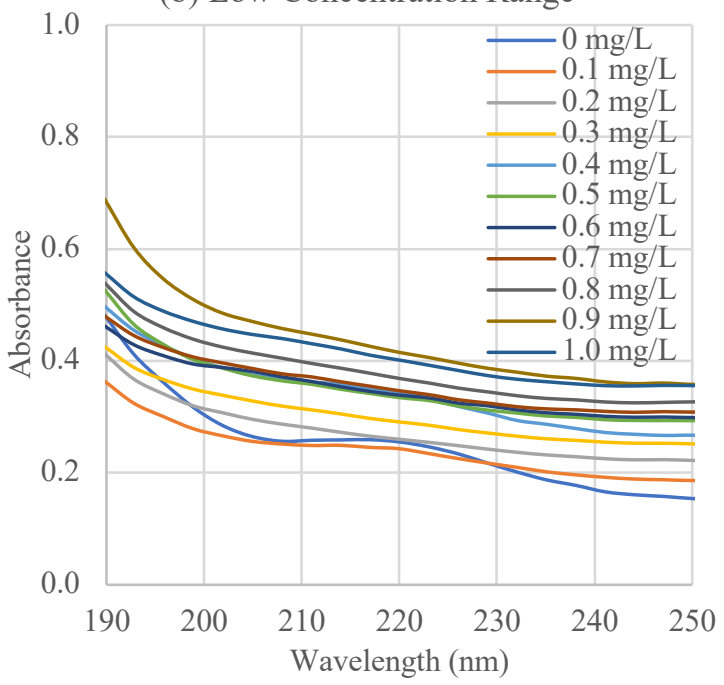

Figure 37: Absorbance spectra of Magnafloc LT27AG polymer in deionized water dosed with Actiflo $\Theta$ enhanced coagulation process chemicals using an in-line spectrophotometer with a $4 \mathrm{~mm}$ flow cell in the concentration ranges of (a) $0-3 \mathrm{mg} / \mathrm{L}$ and (b) $0-1 \mathrm{mg} / \mathrm{L}$

From observing Figures 36 and 37 it is apparent that the absorbance spectra maintain a peak absorbance at a $190 \mathrm{~nm}$ wavelength despite the presence of the Actiflo ${ }^{\circledR}$ process chemicals in the deionized water samples. This trend was observed at both the high and low polymer concentration ranges using both the desktop spectrophotometer, Figures 36a and $36 \mathrm{~b}$, as well as the in-line spectrophotometer, Figures $37 \mathrm{a}$ and $37 \mathrm{~b}$. As observed in previous experimental phases, there were no significant deviations in absorbance beyond $250 \mathrm{~nm}$, which is why these values are not included in the results. In order to accurately model the full-scale Actiflo ${ }^{\circledR}$ enhanced coagulation treatment process, it was also essential 
to work with raw water samples from a natural source. For this purpose, surface water collected from the Rideau River with a turbidity of $32 \mathrm{NTU}$ was used to prepare $400 \mathrm{~mL}$ samples following the exact same procedure as was performed for the deionized water samples. Figures $38 \mathrm{a}$ and $38 \mathrm{~b}$ below represent the absorbance spectra for sample supernatant in the $0-3 \mathrm{mg} / \mathrm{L}$ and $0-1 \mathrm{mg} / \mathrm{L}$ polymer concentration ranges, respectfully, using the desktop spectrophotometer in Rideau River water samples dosed with all Actiflo ${ }^{\circledR}$ process chemicals.

(a) High Concentration Range

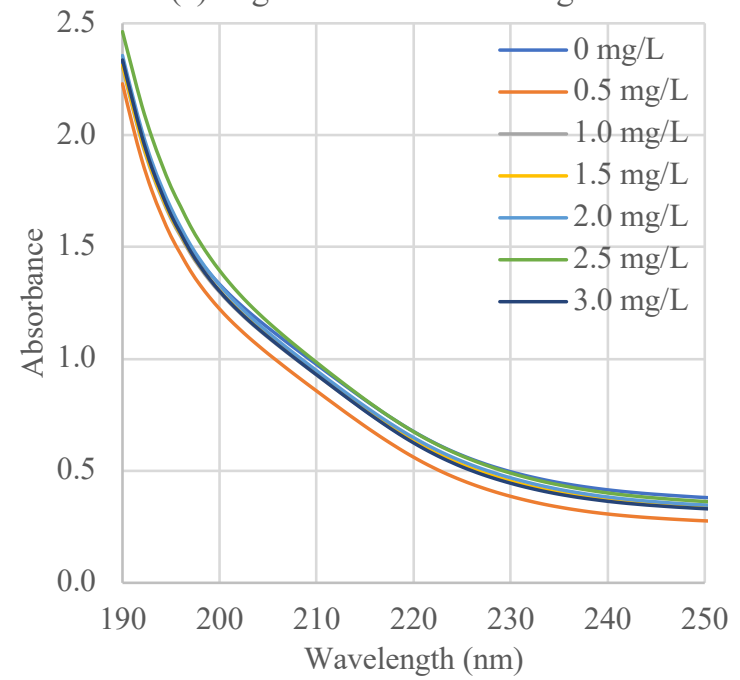

(b) Low Concentration Range

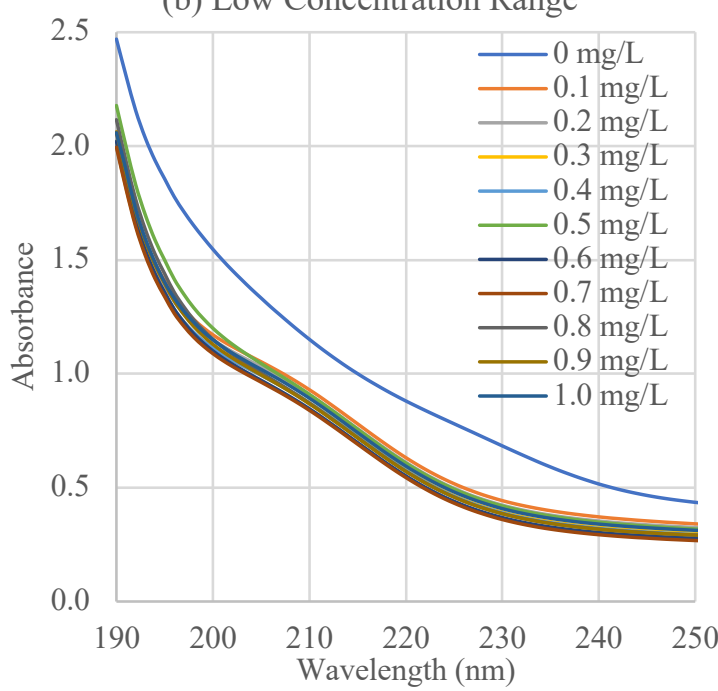

Figure 38: Absorbance spectra of Magnafloc LT27AG polymer in Rideau River (32.0 NTU) water dosed with Actiflo $\mathbb{B}$ enhanced coagulation process chemicals using a desktop spectrophotometer with a $10 \mathrm{~mm}$ flow cell in the concentration ranges of (a) $0-3 \mathrm{mg} / \mathrm{L}$ and (b) $0-1 \mathrm{mg} / \mathrm{L}$

Figures 39a and 39b below represent the absorbance spectra for sample supernatant in the $0-3 \mathrm{mg} / \mathrm{L}$ and $0-1 \mathrm{mg} / \mathrm{L}$ polymer concentration ranges, respectfully, using the in-line spectrophotometer in the same Rideau River water samples as above dosed with all Actiflo ${ }^{\circledR}$ process chemicals. 
(a) High Concentration Range

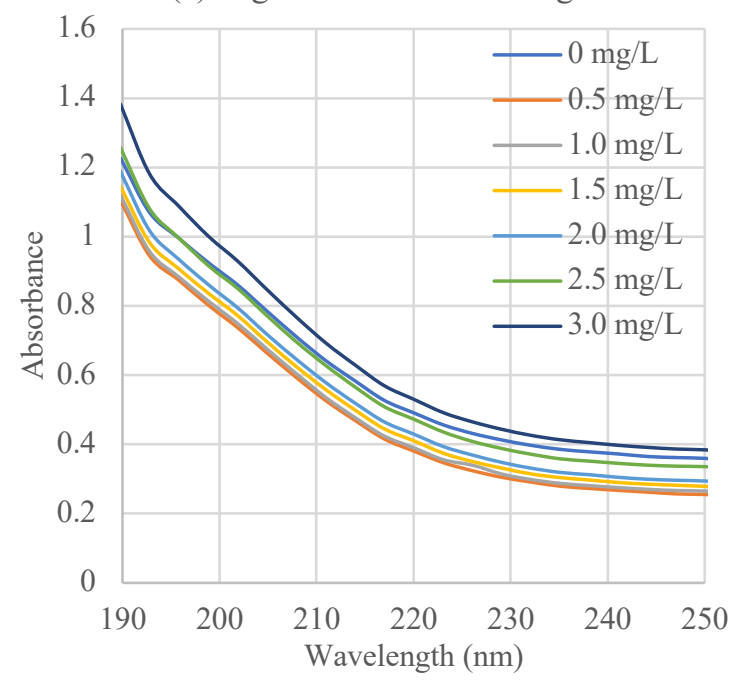

(b) Low Concentration Range

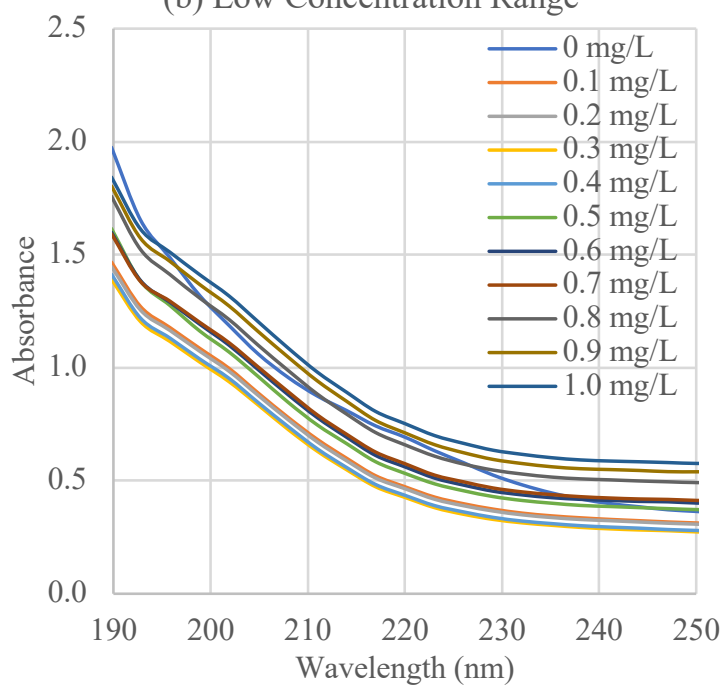

Figure 39: Absorbance spectra of Magnafloc LT27AG polymer in Rideau River water (32.0 NTU) dosed with Actiflo $B$ enhanced coagulation process chemicals using an in-line spectrophotometer with a $4 \mathrm{~mm}$ flow cell in the concentration ranges of (a) $0-3 \mathrm{mg} / \mathrm{L}$ and (b) $0-1 \mathrm{mg} / \mathrm{L}$

Again, the results from the samples prepared with Rideau River water produce an absorbance spectrum following the expected shape with a peak absorbance at 190nm. This trend was observed at both the high and low polymer concentration ranges using both the desktop spectrophotometer, Figures $38 \mathrm{a}$ and $38 \mathrm{~b}$, as well as the in-line spectrophotometer, Figures 39a and 39b. The peak absorbance values at 190nm were significantly higher for each polymer concentration than those reported in the deionized water samples, as is expected due to the increase in turbidity and the particulate matter in the raw water increasing the base absorbance of the sample.

A potential issue with the addition of the Actiflo ${ }^{\circledR}$ enhanced coagulation process chemicals would be their contribution to the peak absorbance value at $190 \mathrm{~nm}$ compared to that of the polymer itself. In order to further investigate this potential impact, two samples of deionized water were dosed with the required dose of each potassium permanganate $(1.0$ 
$\mathrm{mg} / \mathrm{L})$ and the PASS-10 coagulant $(50 \mathrm{mg} / \mathrm{L})$. Each sample was mixed for two minutes using a jar tester at 125RPM and absorbance measurements were taken using both the desktop and in-line spectrophotometers. Figures $40 \mathrm{a}$ and $40 \mathrm{~b}$ below represent the absorbance spectrum for these doses of potassium permanganate as well as PASS-10 coagulant, respectively, in the deionized water samples using a desktop spectrophotometer.

(a) Potassium Permanganate

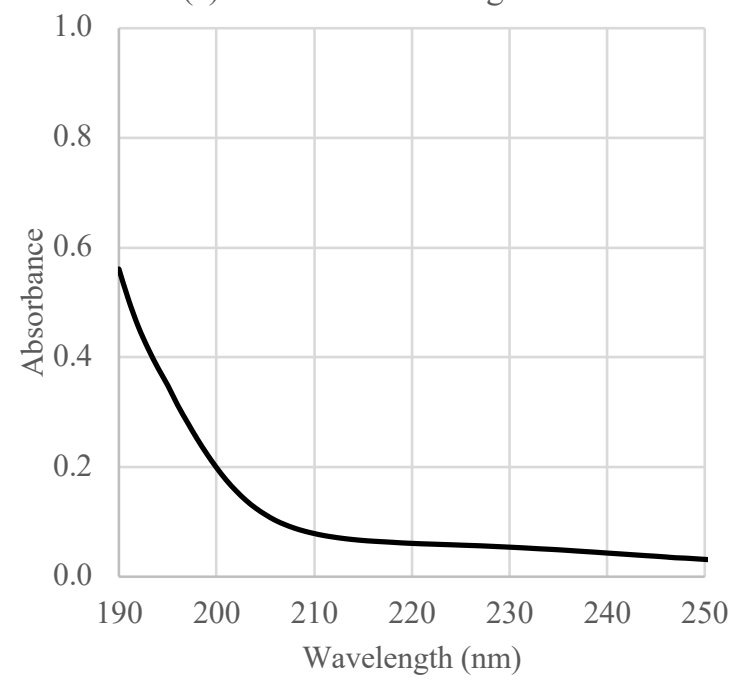

(b) PASS-10 Coagulant

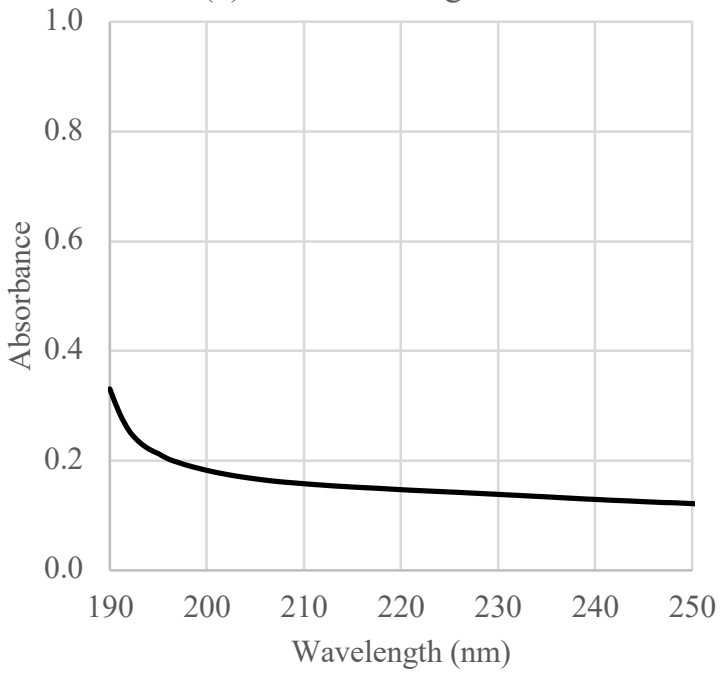

Figure 40: Absorbance spectra of (a) potassium permanganate and (b) PASS-10 coagulant in deionized water using a desktop spectrophotometer and a $10 \mathrm{~mm}$ flow cell

Figures $41 \mathrm{a}$ and $41 \mathrm{~b}$ below represent the absorbance spectrum for these doses of potassium permanganate as well as PASS-10 coagulant, respectively, in the same deionized water samples as above using an in-line spectrophotometer. 
(a) Potassium Permanganate

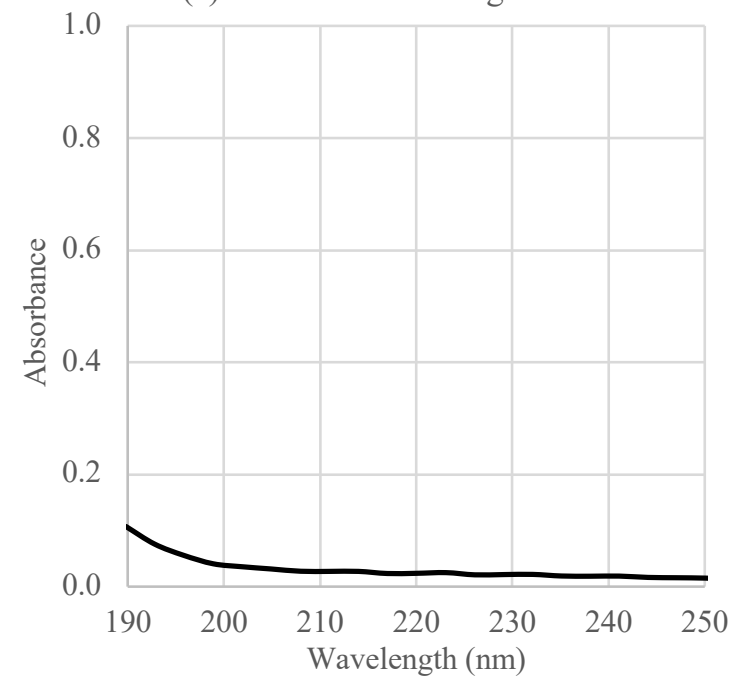

(b) PASS-10 Coagulant

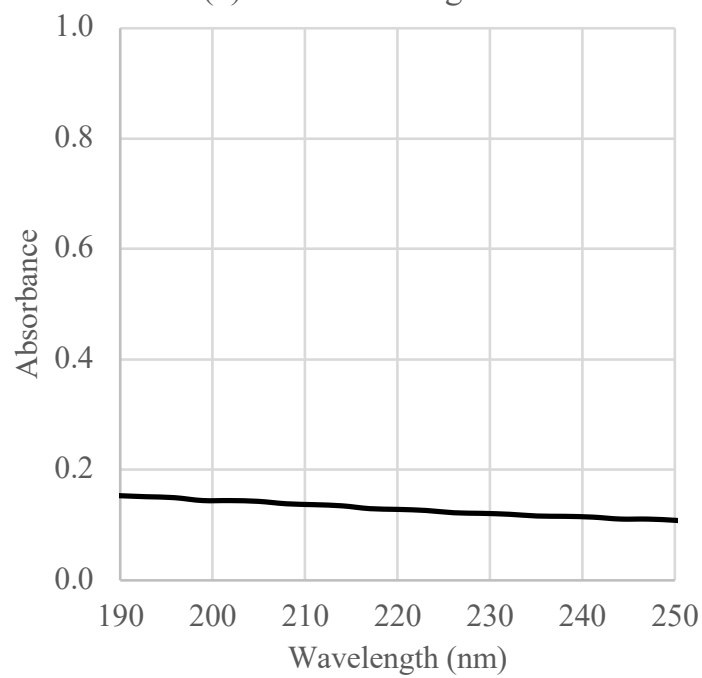

Figure 41: Absorbance spectra of (a) potassium permanganate and (b) PASS-10 coagulant in deionized water using a in-line spectrophotometer and a $4 \mathrm{~mm}$ flow cell

From Figure 40a, the desktop spectrophotometer reports the peak absorbance of potassium permanganate in the deionized water sample at $190 \mathrm{~nm}$ to be $0.5610 \mathrm{AU}$. When comparing this value to that of the lowest polymer concentration $(0.10 \mathrm{mg} / \mathrm{L})$ in deionized water containing all Actiflo ${ }^{\circledR}$ process chemicals, as reported in Figure 36b, the polymer containing sample reports a similar but slightly lower value of $0.5300 \mathrm{AU}$ using the desktop unit. Analyzing the peak absorbance values at $190 \mathrm{~nm}$ of each polymer concentration in the low range, represented by Figure 36b, the desktop unit is not able to differentiate between polymer concentrations as they increase when the Actiflo® process chemicals are present seeing that the potassium permanganate contributes to the peak absorbance more so than the polymer itself. This infers that the desktop unit would not be a reliable method of detecting residual polymer at a concentration of $3.0 \mathrm{mg} / \mathrm{L}$ or lower when potassium permanganate is present at the above-mentioned dosage. Comparatively, from Figure 41a, the in-line spectrophotometer reports the peak absorbance of potassium permanganate in deionized water at $190 \mathrm{~nm}$ to be $0.1074 \mathrm{AU}$. When comparing this value to that of the lowest 
polymer concentration $(0.10 \mathrm{mg} / \mathrm{L})$ in deionized water containing all Actiflo ${ }^{\circledR}$ process chemicals, as reported in Figure 36b, the polymer containing sample reports a much higher value of $0.3640 \mathrm{AU}$. This infers that the in-line spectrophotometer is sensitive enough to detect a significant difference in absorbance between potassium permanganate alone, and with residual polymer present in solution at concentrations as low as $0.10 \mathrm{mg} / \mathrm{L}$.

From Figure 40b, the desktop spectrophotometer reports the peak absorbance of PASS-10 coagulant in deionized water at $190 \mathrm{~nm}$ to be 0.3308 AU. Again, when comparing this value to that of the lowest polymer concentration $(0.10 \mathrm{mg} / \mathrm{L})$ in deionized water containing all Actiflo ${ }^{\circledR}$ process chemicals, as reported in Figure 36b, the polymer containing sample reports a much higher value of 0.5300 AU using the desktop unit. Similarly, when analyzing Figure 41b, the in-line spectrophotometer reports the peak absorbance of PASS10 coagulant in deionized water at $190 \mathrm{~nm}$ to be $0.1534 \mathrm{AU}$. Comparing this again to the peak absorbance at $190 \mathrm{~nm}$ of the lowest polymer concentration $(0.10 \mathrm{mg} / \mathrm{L})$ in deionized water containing all Actiflo ${ }^{\circledR}$ process chemicals, Figure 37b, the in-line unit reports a peak absorbance value of 0.3640 A.U. Since the absorbance value of the PASS-10 coagulant in deionized water is significantly lower than that of the polymer containing sample as read by both spectrophotometers, it can be concluded that the PASS-10 coagulant does not impact the ability of either spectrophotometer to detect residual polymer in a solution containing both constituents. 


\subsubsection{Linear correlations}

As was done for all other absorbance data gathered during the experimental procedure, linear correlations of peak absorbance data versus polymer concentration from Figures 36 to 39 were plotted in order to further analyze the absorbance-polymer concentration relationship and determine the impact that Actiflo process chemicals present in raw water may have on UV-vis spectroscopy sensitivity for residual polymer detection. Figures 42 a and $42 \mathrm{~b}$ below represent the linear correlations for the $0-3 \mathrm{mg} / \mathrm{L}$ and $0-1 \mathrm{mg} / \mathrm{L}$ polymer concentration ranges, respectively, using the desktop spectrophotometer in samples of deionized water containing all Actiflo $®$ enhanced coagulation process chemicals.

(a) High Concentration Range

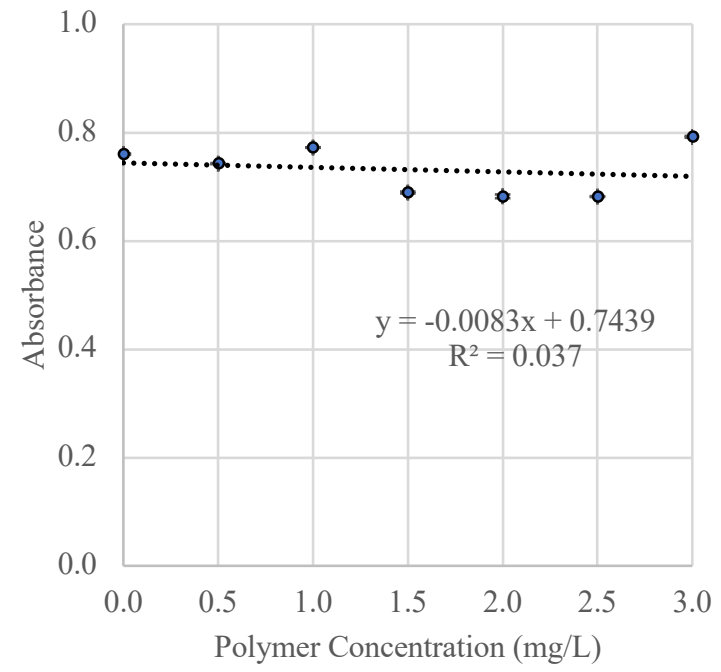

(b) Low Concentration Range

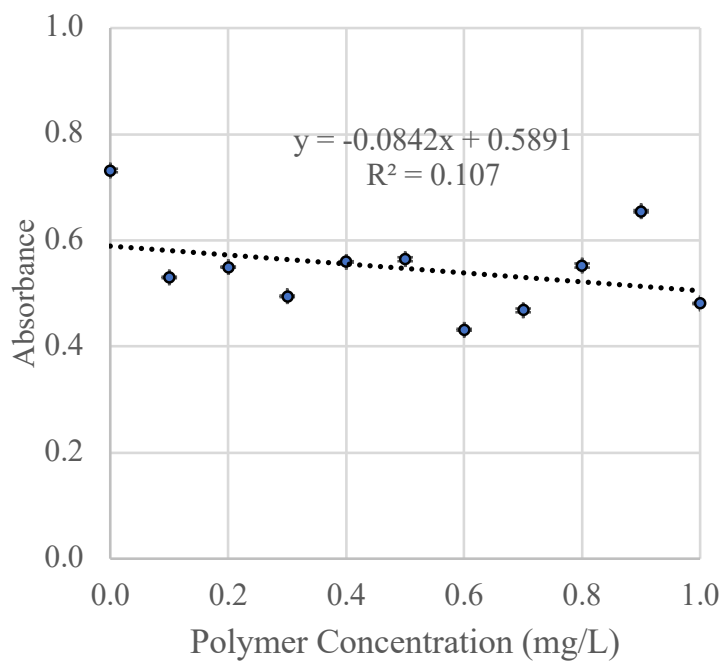

Figure 42: Linear correlation of Magnafloc LT27AG polymer in deionized water dosed with Actiflo $\Theta$ enhanced coagulation process chemicals using a desktop spectrophotometer with a $10 \mathrm{~mm}$ flow cell in the concentration ranges of (a) $0-3 \mathrm{mg} / \mathrm{L}$ and (b) $0-1 \mathrm{mg} / \mathrm{L}$

Figures $43 \mathrm{a}$ and $43 \mathrm{~b}$ below represent the linear correlations for the $0-3 \mathrm{mg} / \mathrm{L}$ and $0-$ $1 \mathrm{mg} / \mathrm{L}$ polymer concentration ranges, respectively, using the in-line spectrophotometer in 
the same samples of deionized water containing all Actiflo® enhanced coagulation process chemicals.

(a) High Concentration Range

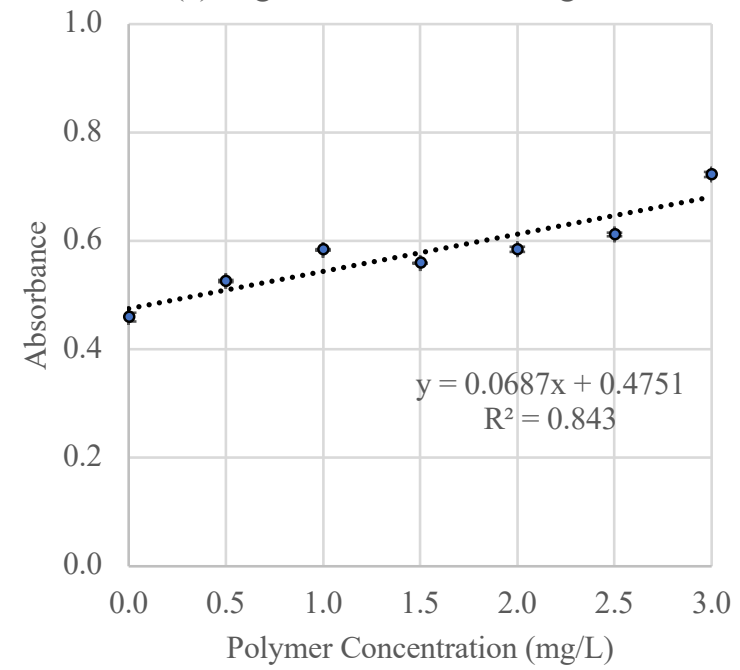

(b) Low Concentration Range

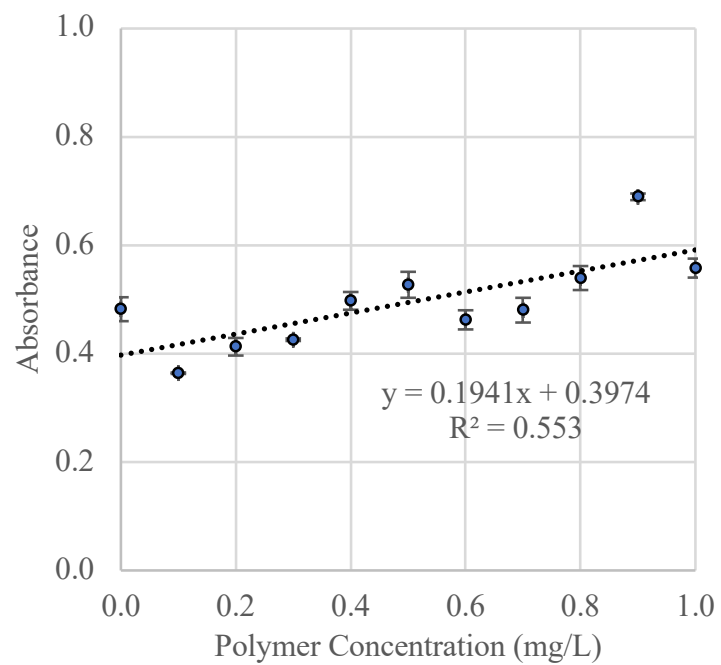

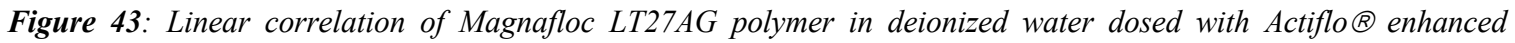
coagulation process chemicals using an in-line spectrophotometer with a $4 \mathrm{~mm}$ flow cell in the concentration ranges of (a) $0-3 \mathrm{mg} / \mathrm{L}$ and (b) $0-1 \mathrm{mg} / \mathrm{L}$

From Figures $42 \mathrm{a}$ and $42 \mathrm{~b}$, it is apparent that the desktop spectrophotometer was unable to form any meaningful correlation between polymer concentration and absorbance at either the high or low polymer concentration range in deionized water when Actiflo ${ }^{\circledR}$ process chemicals were present. As polymer concentration increases, the absorbance values reported by the desktop unit either remain fairly constant or decrease slightly forming a negative correlation overall. The coefficient of determinations for both polymer concentration ranges were also very weak, $\mathrm{R}^{2}=0.033$ for the high concentration range and $\mathrm{R}^{2}=0.107$ for the low concentration range. This infers that the desktop unit does not have the required sensitivity to accurately detect the absorbance value of residual polymer at concentrations of $3.0 \mathrm{mg} / \mathrm{L}$ or lower in the presence of Actiflo ${ }^{\circledR}$ process chemicals. Comparatively, Figures $43 \mathrm{a}$ and $43 \mathrm{~b}$ demonstrate that the in-line spectrophotometer was 
able to form a meaningful positive correlation between polymer concentration and absorbance in deionized water with Actiflo ${ }^{\circledR}$ process chemicals present. At the high concentration range, Figure 43a, this positive linear correlation is quite strong a coefficient of determination of $\mathrm{R}^{2}=0.843$, despite the presence of additional chemicals in solution. At the lower concentration range, Figure 43b, this correlation is still present but slightly weaker, as represented by the coefficient of determination of $\mathrm{R}^{2}=0.553$.

Figures $44 \mathrm{a}$ and $44 \mathrm{~b}$ below represent the linear correlations for the $0-3 \mathrm{mg} / \mathrm{L}$ and $0-$ $1 \mathrm{mg} / \mathrm{L}$ polymer concentration ranges, respectively, using the desktop spectrophotometer in samples of Rideau River water with a turbidity of 32 NTU containing all Actiflo ${ }^{\circledR}$ enhanced coagulation process chemicals.

(a) High Concentration Range

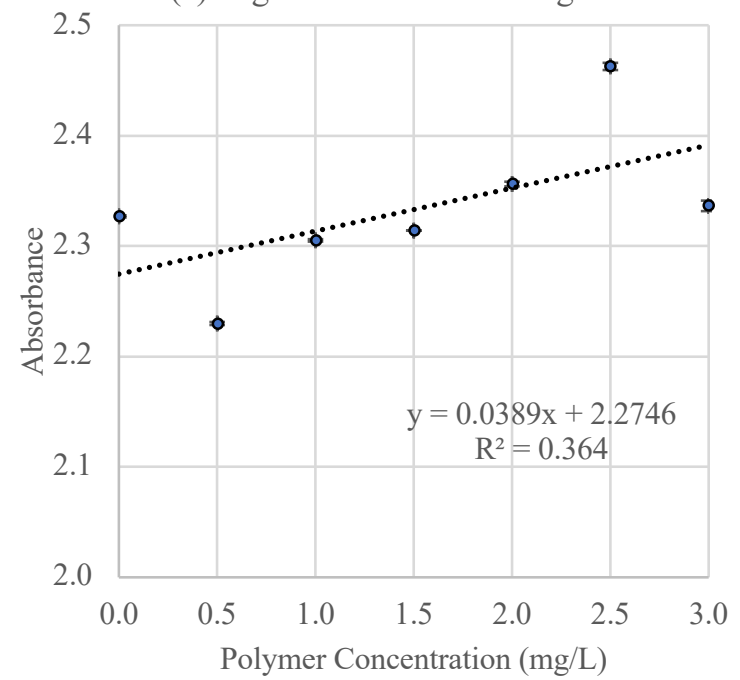

(b) Low Concentration Range

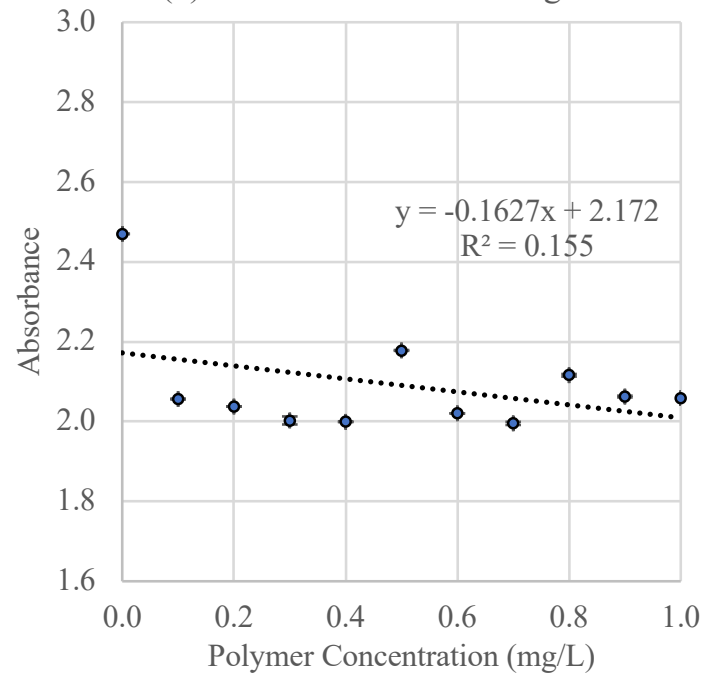

Figure 44: Linear correlation of Magnafloc LT27AG polymer in Rideau River (32.0 NTU) water dosed with Actiflo $\otimes$ enhanced coagulation process chemicals using a desktop spectrophotometer with a $10 \mathrm{~mm}$ flow cell in the concentration ranges of (a) $0-3 \mathrm{mg} / \mathrm{L}$ and (b) $0-1 \mathrm{mg} / \mathrm{L}$ 
Figures $45 \mathrm{a}$ and $45 \mathrm{~b}$ below represent the linear correlations for the $0-3 \mathrm{mg} / \mathrm{L}$ and $0-$ $1 \mathrm{mg} / \mathrm{L}$ polymer concentration ranges, respectively, using the in-line spectrophotometer in samples of the same Rideau River water sample as above with a turbidity of $32 \mathrm{NTU}$ containing all Actiflo ${ }^{\circledR}$ enhanced coagulation process chemicals.

(a) High Concentration Range

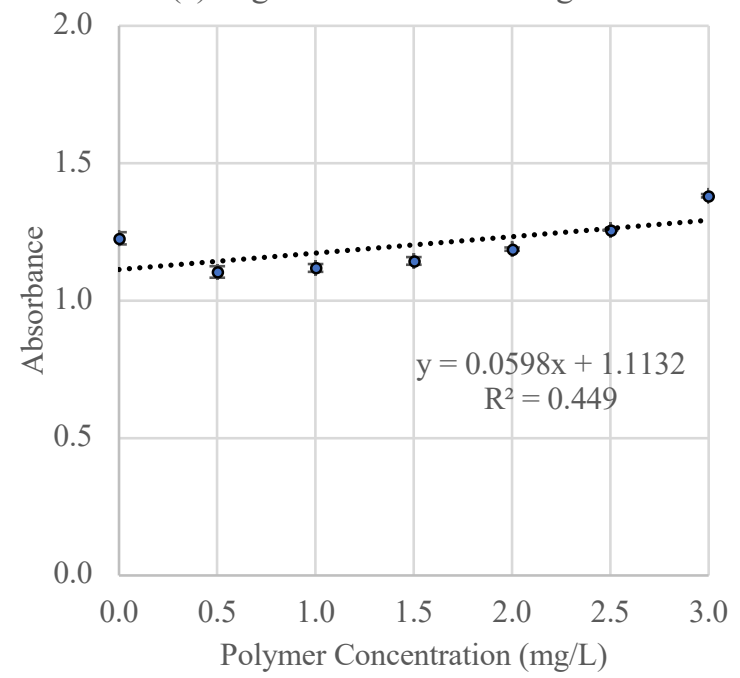

(b) Low Concentration Range

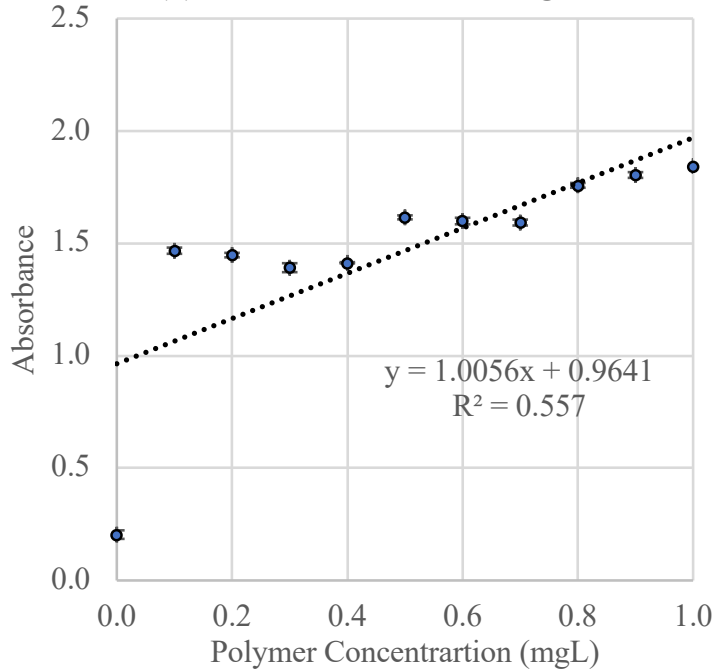

Figure 45: Linear correlation of Magnafloc LT27AG polymer in Rideau River (32.0 NTU) water dosed with Actiflo $B$ enhanced coagulation process chemicals using an in-line spectrophotometer with a $4 \mathrm{~mm}$ flow cell in the concentration ranges of (a) $0-3 \mathrm{mg} / \mathrm{L}$ and (b) $0-1 \mathrm{mg} / \mathrm{L}$

From Figures $44 \mathrm{a}$ and $44 \mathrm{~b}$, it is clear to see that the desktop spectrophotometer again was unable to detect the increase in polymer concentration in the presence of Actiflo ${ }^{\circledR}$ process chemicals present in the Rideau River water sample. This is depicted by the lack of correlation between polymer concentration and absorbance at both polymer concentration ranges, with the high range indicating a coefficient of determination of $\mathrm{R}^{2 .}=0.364$ and the low range being $\mathrm{R}^{2}=0.155$. This is most likely due to the fact that the desktop spectrophotometer does not have the required sensitivity to detect the small concentrations of polymer in the presence of the potassium permanganate, which contributes to quite a 
substantial background absorbance on this unit. Comparatively, from Figures $45 \mathrm{a}$ and $45 \mathrm{~b}$, the in-line unit was still able to form a slightly better positive linear correlation between polymer concentration and absorbance in the presence of Actiflo ${ }^{\circledR}$ process chemicals in a Rideau River water sample. The higher polymer concentration range, Figure 45a, showed a substantial decrease in this correlation $\left(\mathrm{R}^{2}=0.449\right)$ when compared to the same sample set in deionized water, as depicted in Figure $43 \mathrm{a}\left(\mathrm{R}^{2}=0.843\right)$. This decrease in correlation is likely due to the increase in particulate matter present in the sample, as indicated by the increase in turbidity, contributing to an increase in background absorbance. The lower polymer concentration range formed a very similar correlation in Rideau River water, Figure $45 \mathrm{~b}$, as the same sample set in deionized water, Figure $43 \mathrm{~b}$. The coefficient of determination values were relatively similar, however the slope of the correlation did increase from 0.1941 in the deionized water sample to 1.0056 in the Rideau River water sample. These results not only indicate that the in-line unit is a more sensitive tool for the detection of residual polymer in drinking water treatment applications when compared to the desktop unit, but also that the correlation between residual polymer concentration and absorbance can still be detected at very low concentrations in the presence of additional process chemicals in raw water matrices. 


\subsection{Determination of optimum polymer dose in drinking water treatment using}

$U V$-vis spectroscopy

Two bulk water samples, one measuring a high turbidity and one measuring a low turbidity, were collected from the Rideau River in Ottawa, Ontario. Each sample was dosed with polymer, a Magnafloc LT27AG anionic polymer and a Hydrex 3661 cationic polymer, in concentration ranges both overdosing and under-dosing the optimum polymer dosage. The optimum polymer dose for each water sample was pre-determined using a preliminary settling test, and turbidity measurements of the supernatant were taken of each sample dosed with polymer concentrations within the optimum dose range. The samples were left to settle for a period of time relative to that required for the optimal dose to reach visible clarity, and the supernatant was then collected and used for UV-vis absorbance measurements on both an in-line spectrophotometer and a desktop spectrophotometer. A relationship between the optimum polymer dose and the supernatant absorbance at 190nm was established for both polymer types in both the high and low turbidity bulk water samples. In addition to this, a particle counter was also used to measure the particle counts of each sample of supernatant for each data set. This was done in order to further analyze the data and determine whether or not trends in the particle data corresponded to those of the turbidity data, as well as the absorbance data. In addition to this, the effect of Actiflo ${ }^{\circledR}$ enhanced coagulation process chemicals on the determination of optimum dose using UVvis spectroscopy was also explored. 


\subsubsection{Magnafloc LT27AG Polymer}

\subsubsection{Settling tests}

Magnafloc LT27AG is an anionic polyacrylamide polymer with an ultra-high molecular weight and a medium charge density. A preliminary settling test was performed on each different water matrices in order to determine the optimum polymer dose range based on apparent clarity of the supernatant and the fastest settling time. The samples, prepared in graduated cylinders, were overdosed and under-dosed with polymer relative to this optimum dose range and left to settle whilst being observed and timed simultaneously. Once a particular sample supernatant reached apparent clarity, the time was recorded, and this sample was assumed to contain the optimum polymer dosage. At this time, the supernatant of all other samples was collected to be used for turbidity measurements, and the interfaces of the sediment in each sample were also measured and recorded. Tables 13, 14 and 15 below represent the results of the settling tests for the high turbidity Rideau River water, the low turbidity Rideau River water, and the high turbidity Rideau River water also dosed with Actiflo® enhanced coagulation process chemicals.

Table 13: Settling test data for high turbidity (9172 NTU) Rideau River Water dosed with Magnafloc LT27AG polymer

\begin{tabular}{ccc}
\hline $\begin{array}{c}\text { Polymer Concentration } \\
(\mathbf{m g} / \mathbf{L})\end{array}$ & Interface $(\mathbf{m m})$ & $\begin{array}{c}\text { Supernatant Turbidity } \\
(\mathbf{N T U})\end{array}$ \\
\hline 0.00 & N/A & 9172 \\
\hline 0.10 & 2.0 & 595 \\
\hline 0.50 & 2.0 & 252 \\
\hline 1.00 & 2.0 & 185 \\
\hline 1.50 & 3.0 & 157 \\
\hline 2.00 & 6.0 & 140 \\
\hline 3.00 & 6.0 & 178 \\
\hline 4.00 & 3.0 & 190 \\
\hline 5.00 & 4.0 & 209 \\
\hline Optimum Settling Time & 170 seconds at $2.00 \mathrm{mg} / \mathrm{L}$ polymer dose \\
\hline
\end{tabular}


Table 14: Settling test data for low turbidity (260 NTU) Rideau River Water dosed with Magnafloc LT27AG polymer

\begin{tabular}{ccc}
\hline $\begin{array}{c}\text { Polymer Concentration } \\
(\mathbf{m g} / \mathbf{L})\end{array}$ & Interface $(\mathbf{m m})$ & $\begin{array}{c}\text { Supernatant Turbidity } \\
\text { (NTU) }\end{array}$ \\
\hline 0.00 & N/A & 260 \\
\hline 0.05 & $\sim 1.0$ & 107 \\
\hline 0.10 & $\sim 1.0$ & 108 \\
\hline 0.50 & $\sim 1.0$ & 113 \\
\hline 1.00 & $\sim 1.0$ & 114 \\
\hline 1.50 & $\sim 1.0$ & 115 \\
\hline 2.00 & $\sim 1.0$ & 110 \\
\hline 3.00 & $\sim 1.0$ & 125 \\
\hline Optimum Settling Time & 160 seconds at $0.05 \mathrm{mg} / \mathrm{L}$ polymer dose \\
\hline
\end{tabular}

Table 15: Settling test data for high turbidity (9000 NTU) Rideau River Water dosed Actiflo $®$ enhanced coagulation process chemicals and Magnafloc LT27AG polymer

\begin{tabular}{ccc}
\hline $\begin{array}{c}\text { Polymer Concentration } \\
(\mathbf{m g} / \mathbf{L})\end{array}$ & Interface (mm) & $\begin{array}{c}\text { Supernatant Turbidity } \\
(\mathbf{N T U})\end{array}$ \\
\hline 0.00 & N/A & 9000 \\
\hline 0.05 & 2.0 & 980 \\
\hline 0.10 & 2.0 & 965 \\
\hline 0.50 & 4.0 & 385 \\
\hline 1.00 & 3.0 & 373 \\
\hline 2.00 & 3.0 & 392 \\
\hline 4.00 & 2.0 & 462 \\
\hline 6.00 & 2.0 & 516 \\
\hline 8.00 & 2.0 & 525 \\
\hline Optimum Settling Time & 266 seconds at $0.50 \mathrm{mg} / \mathrm{L}$ polymer dose \\
\hline
\end{tabular}

From these preliminary settling test results, the optimum dose of Magnafloc LT27AG polymer water was determined to be $2.00 \mathrm{mg} / \mathrm{L}$ for the high turbidity Rideau River, $0.05 \mathrm{mg} / \mathrm{L}$ for the low turbidity Rideau River water, and $0.50 \mathrm{mg} / \mathrm{L}$ for the high turbidity Rideau River water dosed with Actiflo ${ }^{\circledR}$ enhanced coagulation process chemicals. These results were based on apparent clarity of the supernatant and the fastest settling time. 


\subsubsection{Turbidity measurements}

Samples of Rideau River water of both high and low turbidity were dosed with polymer both overdosing and under-dosing the optimum polymer dose range which was predetermined through a preliminary settling test, as described above. The supernatant of the settled samples was collected and used for turbidity measurements, absorbance measurements as well as particle count measurements. In order to better visualize the relationship between turbidity and polymer dosage, the data was plotted in scatter plot form. Figure 46 below represents the turbidity data in relation to polymer concentration for the supernatant of high turbidity (9172NTU) Rideau River water samples dosed with polymer both over and under-dosing the preliminarily determined optimum dosage range.

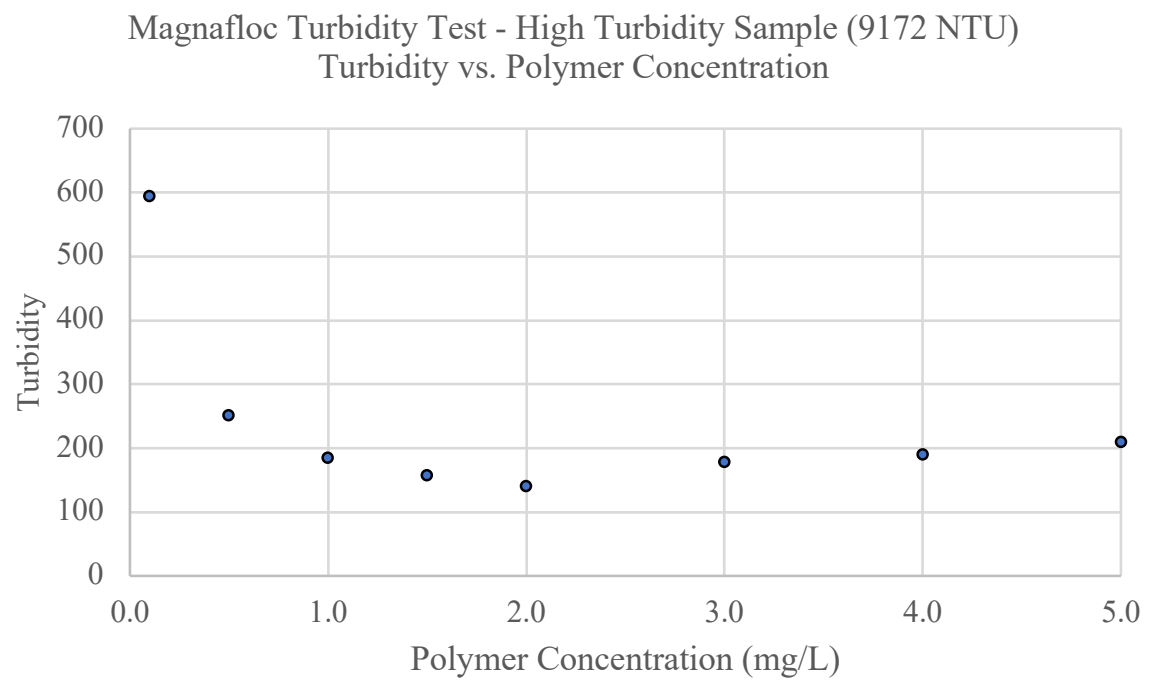

Figure 46: Turbidity values corresponding to Magnafloc LT27AG polymer concentrations within the optimum dose range in the supernatant of a high turbidity (9172NTU) Rideau River water sample 
Figure 47 below represents the turbidity data in relation to polymer concentration for the supernatant of low turbidity (260 NTU) Rideau River water samples dosed with polymer both over and under-dosing the preliminarily determined optimum dosage range.

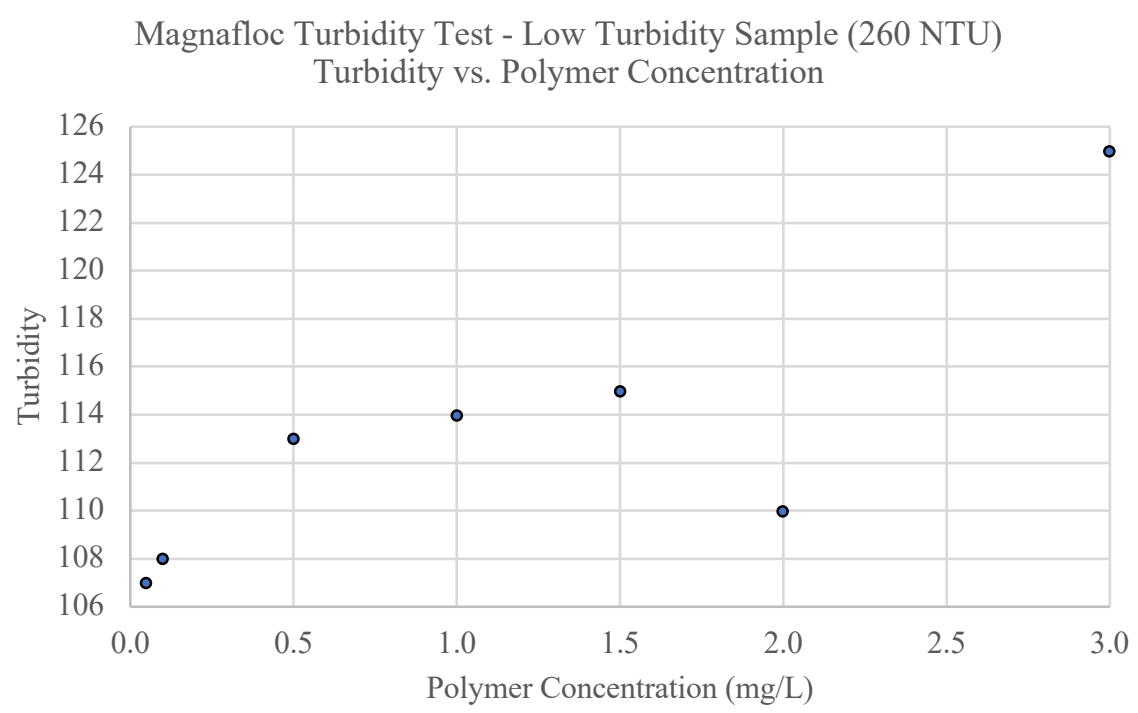

Figure 47: Turbidity values corresponding to Magnafloc LT27AG polymer concentrations within the optimum dose range in the supernatant of a low turbidity (260 NTU) Rideau River water sample

Figure 48 below represents the turbidity data in relation to polymer concentration for the supernatant of high turbidity (9000 NTU) Rideau River water samples dosed with polymer both over and under-dosing the preliminarily determined optimum dosage range. In addition, each sample was also dosed with Actiflo® enhanced coagulation process chemicals in concentrations listed in Table 4 found in Chapter 3: Materials and Methods. 


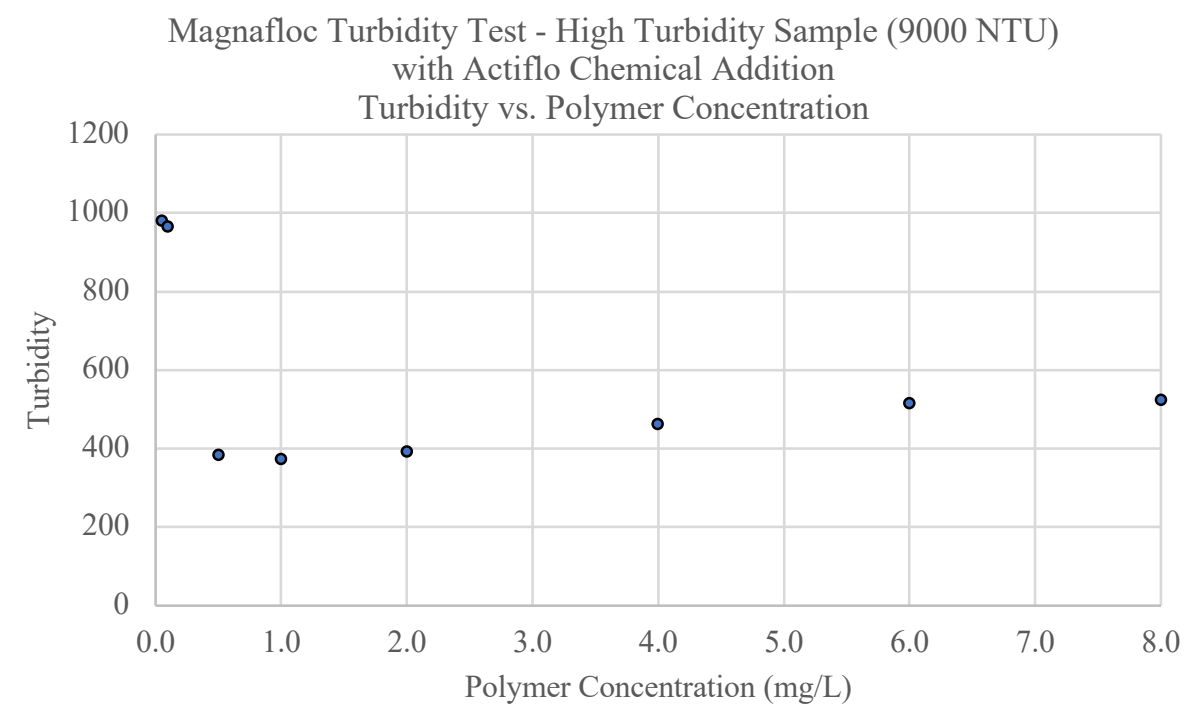

Figure 48: Turbidity values corresponding to Magnafloc LT27AG polymer concentrations within the optimum dose

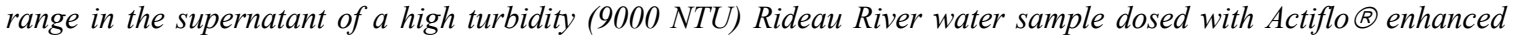
coagulation process chemicals

Figure 46, representing the relationship between turbidity and polymer concentration in the supernatant of a high turbidity (9172 NTU) Rideau River water sample, reports that the minimum turbidity (140 NTU) corresponds to the sample dosed with $2.0 \mathrm{mg} / \mathrm{L}$ of polymer. This polymer concentration is in accordance with the preliminary value determined using the settling test. As was expected, an under-dose of polymer resulted in an increase in turbidity. This is due to the fact that polymer has a finite capacity for absorption; if there is an insufficient concentration of polymer available in the sample solution to properly coagulate the suspended particulates, more particles will remain in the supernatant postsettling and the turbidity will measure a higher value (5). As polymer dosage was increased toward the optimum value, the turbidity decreased accordingly. Again, this is expected seeing that an increase in polymer concentration implies an increase in capacity for absorption. In the overdose range, a slight increase in turbidity was observed, however the values measured only slightly higher than the minimum value associated with the optimum 
dose. This is expected, as the optimum polymer dose corresponds to the concentration of polymer at which the maximum absorbance occurs. Beyond this dosage the polymer is no longer effective, and the removal of suspended particulates no longer increases, meaning the turbidity should remain fairly constant. The slight increase in turbidity can be attributed to the fact that the excess polymer in the overdose range will remain suspended in the supernatant as residual polymer, which contributes slightly to the overall turbidity.

Figure 47, representing the relationship between turbidity and polymer concentration in the supernatant of a low turbidity (260 NTU) Rideau River water sample, reports that the minimum turbidity (107 NTU) corresponds to the sample dosed with $0.05 \mathrm{mg} / \mathrm{L}$ of Magnafloc polymer. The sample dosed with $0.10 \mathrm{mg} / \mathrm{L}$ of polymer is only marginally more turbid (108 NTU). These concentration values coincide with the predetermined settling test estimates. The samples were not under-dosed beyond the value of $0.05 \mathrm{mg} / \mathrm{L}$ as accurately measuring this volume of polymer is extremely difficult due to the viscous solution polymer forms with water. In the overdosed range, the turbidity once again increases with increasing polymer dose. There is the exception of the $2.0 \mathrm{mg} / \mathrm{L}$ dosage, in which case the turbidity decreases suddenly to 110 NTU. This outlier may be due to an issue with the mixing of the bulk sample, which was mixed prior to preparing each sample of different polymer concentration in order to maintain suspension of particles and ensure each individual sample was created with water of equal turbidity. There is the possibility that the raw water collected to prepare the $2.0 \mathrm{mg} / \mathrm{L}$ contained a less evenly distributed number of suspended particles, and in turn a lower initial turbidity than the other samples, leading to a lower turbidity overall after settling. These results indicate that in water matrices of 
lower turbidity, determining the optimum polymer dose using turbidity measurements alone may not be a viable method for accurate results.

Figure 48, representing the relationship between turbidity and polymer concentration in the supernatant of a high turbidity (9000 NTU) Rideau River water sample dosed with Actiflo enhanced coagulation process chemicals, reports that the minimum turbidity (373 NTU) corresponds to a $1.00 \mathrm{mg} / \mathrm{L}$ dosage of polymer. Again, this dosage corresponds to the optimum dose range that was predetermined using an initial settling test. In the under-dose range, the curve once again tends to the previously observed pattern of increased turbidity, which decreases as the polymer concentration is incremented towards optimal concentration. Beyond this optimum dosage, as polymer concentration is further increased into the overdose range, the turbidity follows the previously observed trend of increasing slightly. These results indicate that in highly turbid water matrices containing additional water treatment chemical constituents, the method of determining optimum polymer dose through turbidity measurements has viable potential using Magnafloc LT27AG polymer.

\subsubsection{UV-vis absorbance spectra}

In order to test the viability of using UV-vis spectrophotometry as a method of determining optimum polymer dose in drinking water treatment applications, the optimum dose results of the settling test and turbidity measurements were compared to the absorbance results produced by UV-vis spectroscopy using both a desktop and in-line spectrophotometer. The supernatant of the samples prepared for the settling tests and turbidity measurements was reserved and used for the UV-vis spectroscopy absorbance tests as well. Figures 49a and 
49b below represent the absorbance spectra of the supernatant of high turbidity (9172NTU)

Rideau River water samples dosed with Magnafloc polymer at concentrations both overdosing and under-dosing the optimum polymer dose range using a desktop and in-line spectrophotometer, respectively. Figures 50a and 50b below represent the absorbance spectra of the supernatant of low turbidity (260 NTU) Rideau River water samples dosed with Magnafloc polymer at concentrations both overdosing and under-dosing the optimum polymer dose range using a desktop and in-line spectrophotometer, respectively.

(a) desktop spectrophotometer

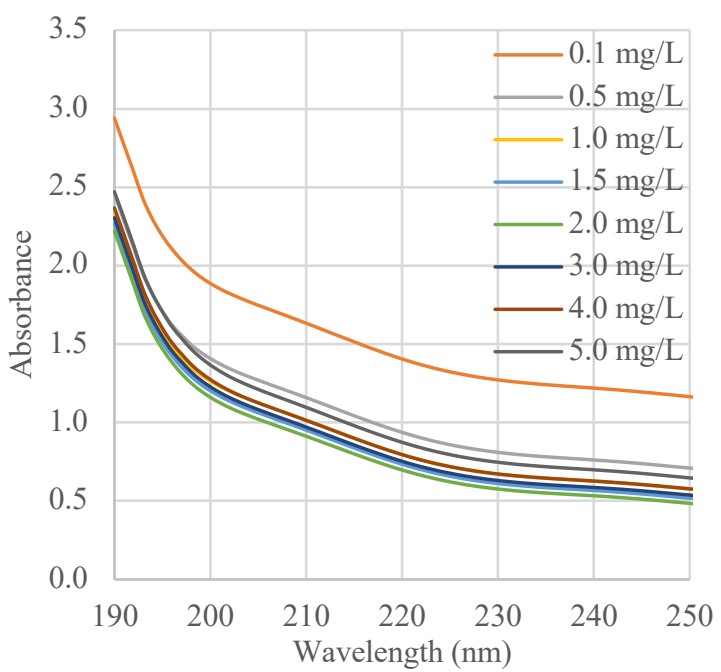

(b) in-line spectrophotometer

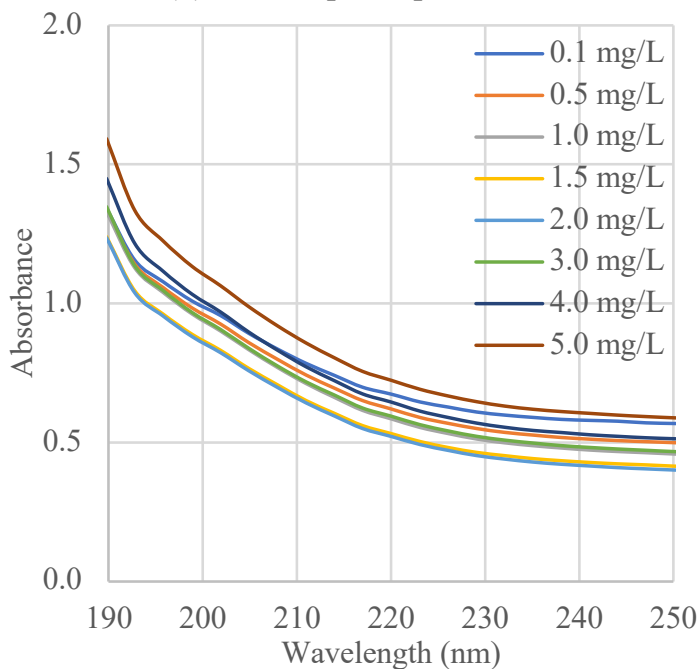

Figure 49: UV-vis absorbance spectra of Magnafloc LT27AG polymer in a sample of high turbidity (9172 NTU) Rideau River water using a (a) desktop spectrophotometer and a (b) in-line spectrophotometer for identifying the samples optimum polymer dose 
(a) desktop spectrophotometer

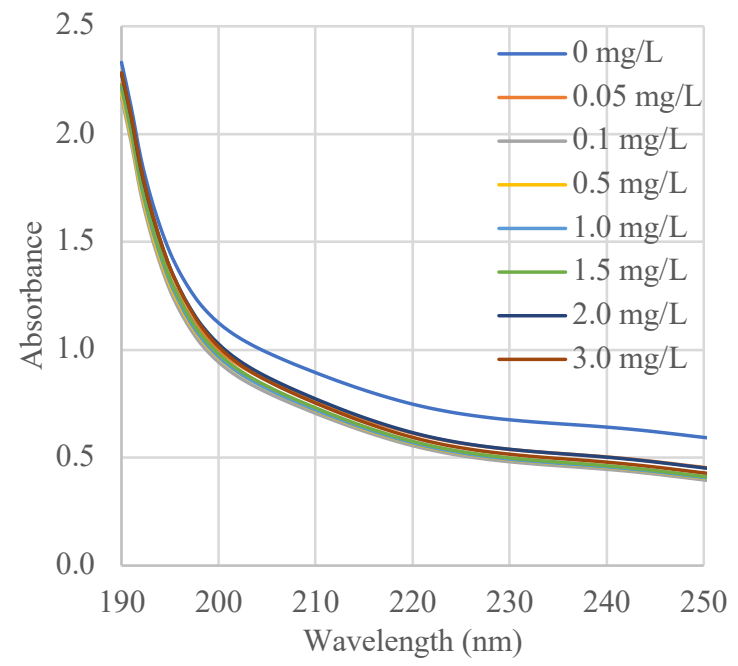

(b) in-line spectrophotometer

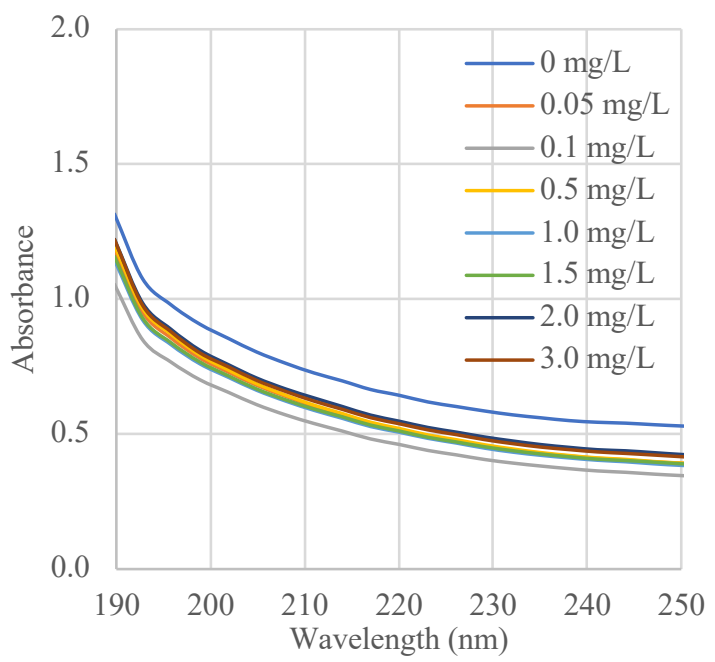

Figure 50: UV-vis absorbance spectra of Magnafloc LT27AG polymer in a sample of low turbidity (260 NTU) Rideau River water using a (a) desktop spectrophotometer and a (b) in-line spectrophotometer for identifying the samples optimum polymer dose

As is observed in Figures 49 and 50, both the high and low turbidity Rideau River water samples resulted in absorbance spectra with the expected shape and peak absorbance at 190nm using both the desktop and in-line spectrophotometers. The peak absorbance values of the high turbidity samples were slightly higher on both spectrophotometers when compared to that of the low turbidity samples, which is expected as the increase in suspended particulates contributes to the absorbance value. There absorbance spectra curves of each different concentration are also more sparsely distributed than those in experimental phases one, two and three. This is also expected seeing that the polymer is overdosed and under-dosed relative to the optimum dose rather than dosed in equal incrementations as has been done for all previous experimental phases.

The same experiment was also conducted with Magnafloc polymer in a high turbidity (9000 NTU) Rideau River water sample dosed with Actiflo ${ }^{\circledR}$ enhanced coagulation 
process chemicals in concentrations reported in Table 4 found in Chapter 3: Materials and Methods. Figures 51a and 51b below represent the absorbance spectra for the supernatant of these high turbidity Rideau River water samples treated with Actiflo ${ }^{\circledR}$ enhanced coagulation process chemicals using both the desktop and in-line spectrophotometers, respectively.

(a) desktop spectrophotometer

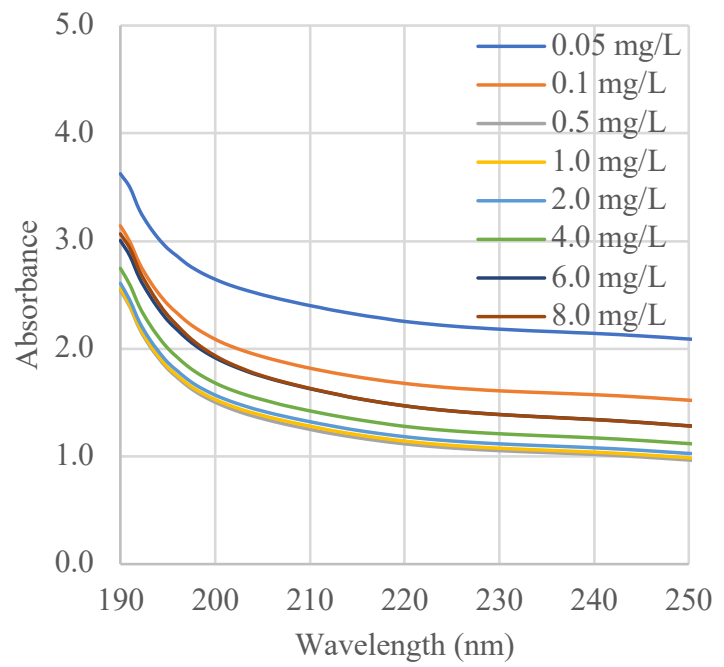

(b) in-line spectrophotometer

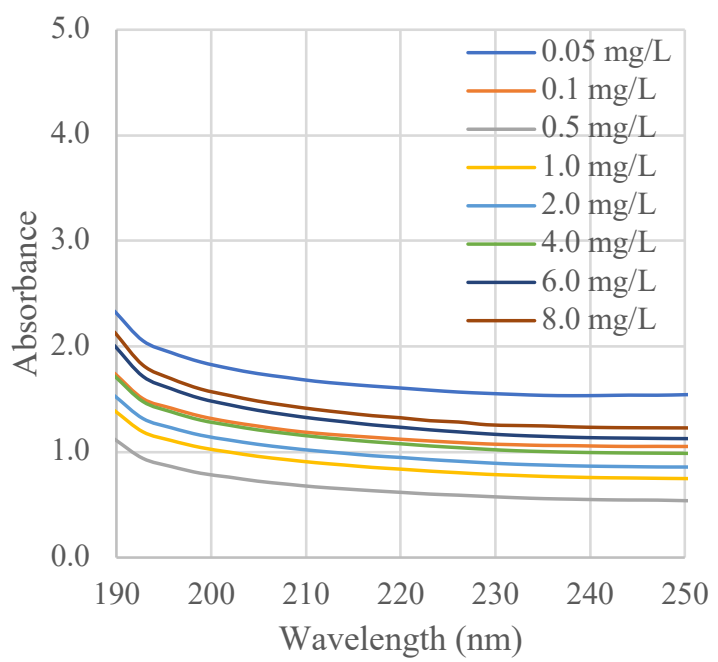

Figure 51: UV-vis absorbance spectra of Magnafloc LT27AG polymer in a sample of high turbidity (9000 NTU) Rideau River water dosed with Actiflo enhanced coagulation process chemicals using a (a) desktop spectrophotometer and a (b) in-line spectrophotometer for identifying the samples optimum polymer dose

Again, from Figure 51 it is apparent that even in the presence of Actiflo ${ }^{\circledR}$ enhanced coagulation process chemicals in a highly turbid water sample, the supernatant of the treated samples resulted in the expected shape and peak absorbance values at 190nm using both the desktop and in-line spectrophotometers. The curves were higher in peak absorbance than those of the samples not containing the Actiflo ${ }^{\circledR}$ process chemicals, which was expected as the chemicals do contribute to some of the overall absorbance of the sample. Regardless of this, a clear increase in absorbance at 190nm as well as an increase 
and decrease in overall absorbance between the overdosed and under-dosed samples, respectively, is still observed in the presence the additional constituents added to each sample. These results, along with those in Figures 49 and 50, demonstrate that the use of UV-vis absorbance as a method for the detection of residual polymer can be replicated in water matrices of both high and low turbidity, with and without the addition of process chemicals.

\subsubsection{Linear correlations}

As was done for all other absorbance data gathered during the previous experimental phases, linear correlations of peak absorbance data versus polymer concentration from Figures 49,50 and 51 were plotted in order to further analyze the absorbance-polymer concentration relationship and determine whether or not UV-vis spectroscopy is a method capable of observing the optimum polymer dose in each different water sample. The linear relationship between the polymer concentration and sample absorbance allows for the construction of a calibration curve to determine unknown polymer concentrations in a given water sample. Figures 52a and 52b below represent the linear correlations of high turbidity (9172 NTU) Rideau River water samples overdosed and under-dosed with Magnafloc polymer relative to the polymers predetermined optimum dosage range using both a desktop and in-line spectrophotometer, respectively. 
(a) desktop spectrophotometer

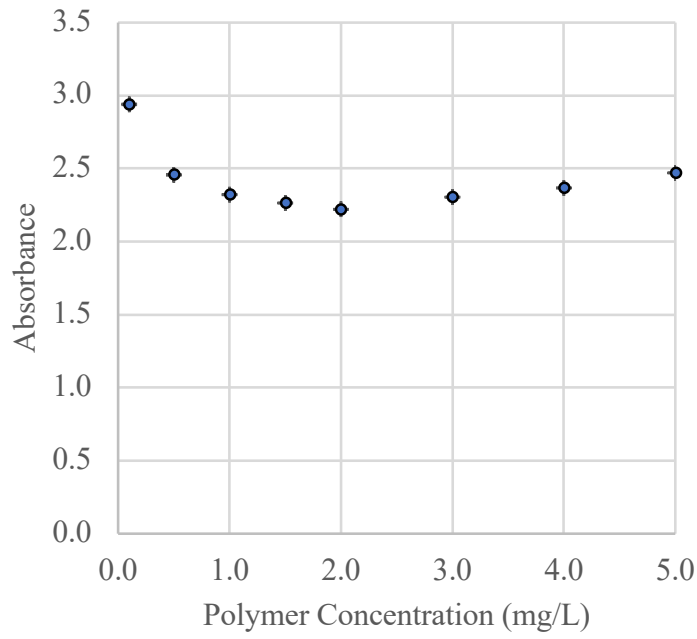

(b) in-line spectrophotometer

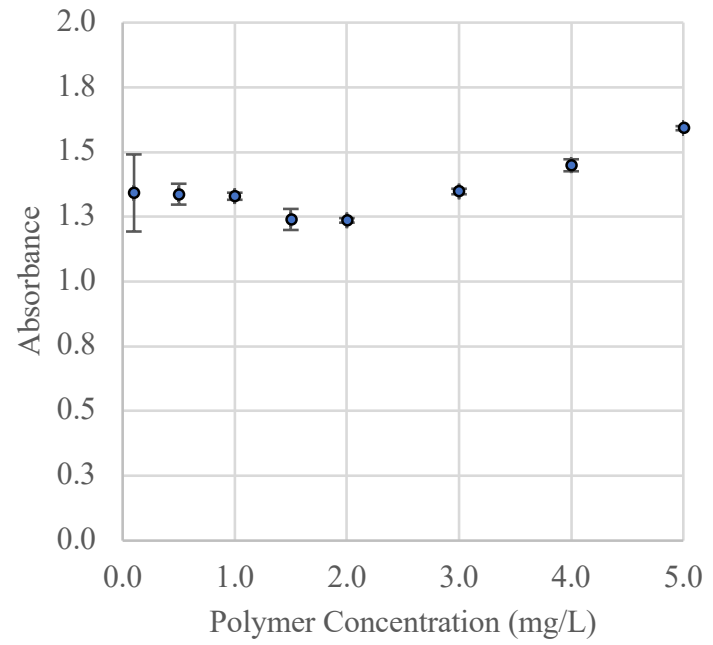

Figure 52: Linear correlation of Magnafloc LT27AG absorbance at 190nm in high turbidity (9172 NTU) Rideau River water using a (a) desktop spectrophotometer and a (b) in-line spectrophotometer for determination of the sample's optimum polymer dose

Figures $53 \mathrm{a}$ and $53 \mathrm{~b}$ below represent the linear correlations of low turbidity (260 NTU)

Rideau River water samples overdosed and under-dosed with Magnafloc polymer relative to the polymers predetermined optimum dosage range using both a desktop and in-line spectrophotometer, respectively.

(a) desktop spectrophotometer

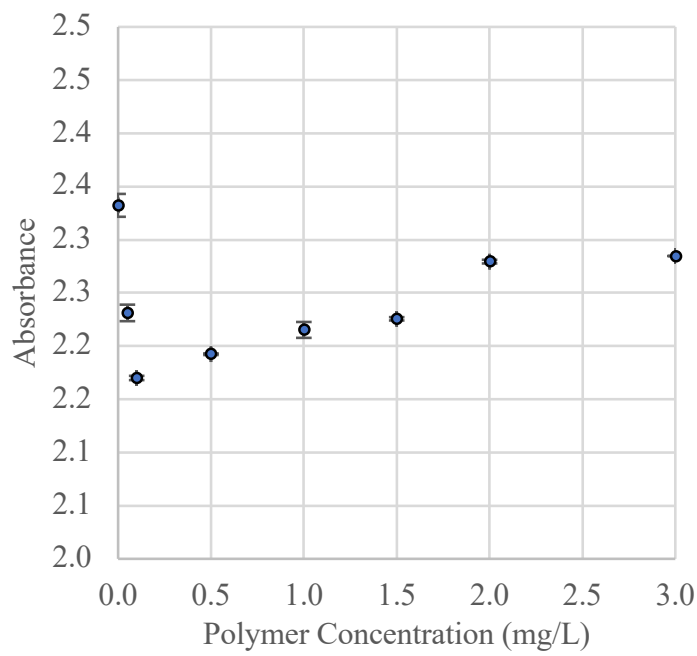

(b) in-line spectrophotometer

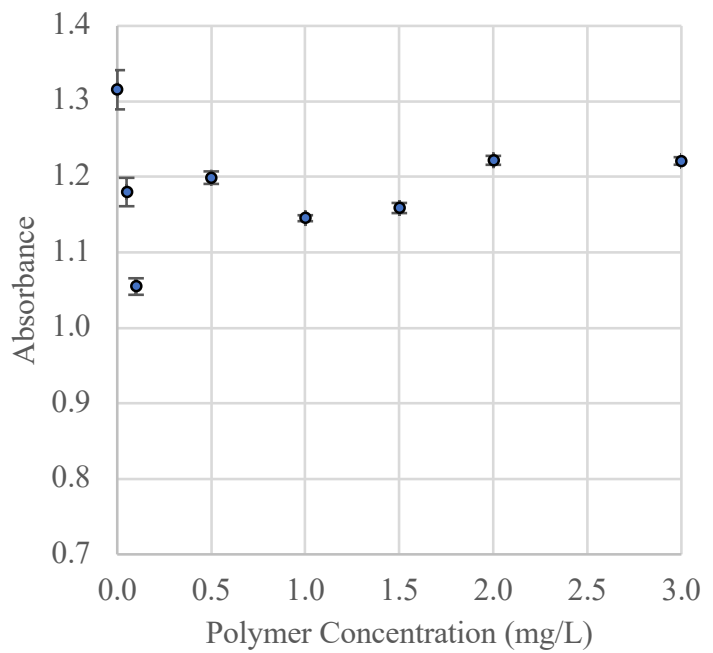

Figure 53: Linear correlation of Magnafloc LT27AG absorbance at 190nm in low turbidity (260 NTU) Rideau River water using a (a) desktop spectrophotometer and a (b) in-line spectrophotometer for determination of the sample's optimum polymer dose 
Figures 54a and 54b below represent the linear correlations of high turbidity (9000 NTU) Rideau River water samples dosed with Actiflo process chemicals using both the desktop and in-line spectrophotometers, respectively. Again, these samples overdosed and underdosed with Magnafloc polymer relative to the polymers predetermined optimum dosage range.

(a) desktop spectrophotometer

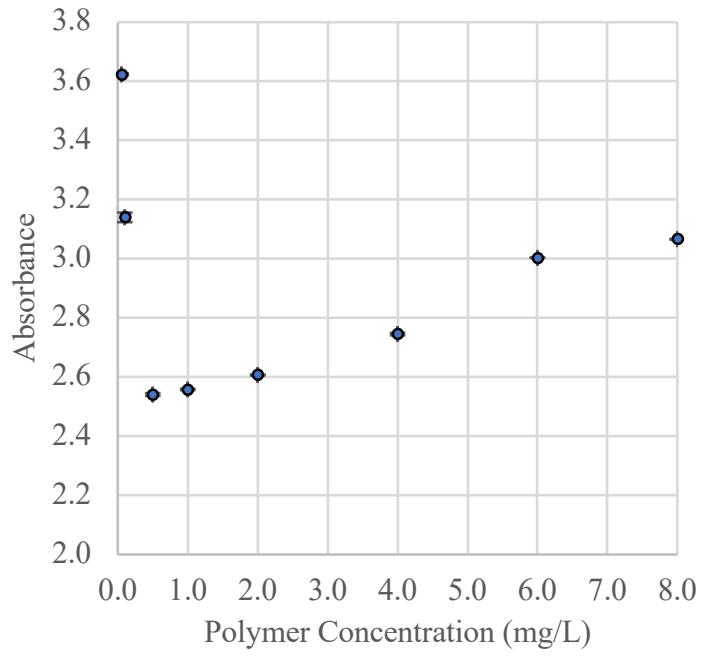

(b) in-line spectrophotometer

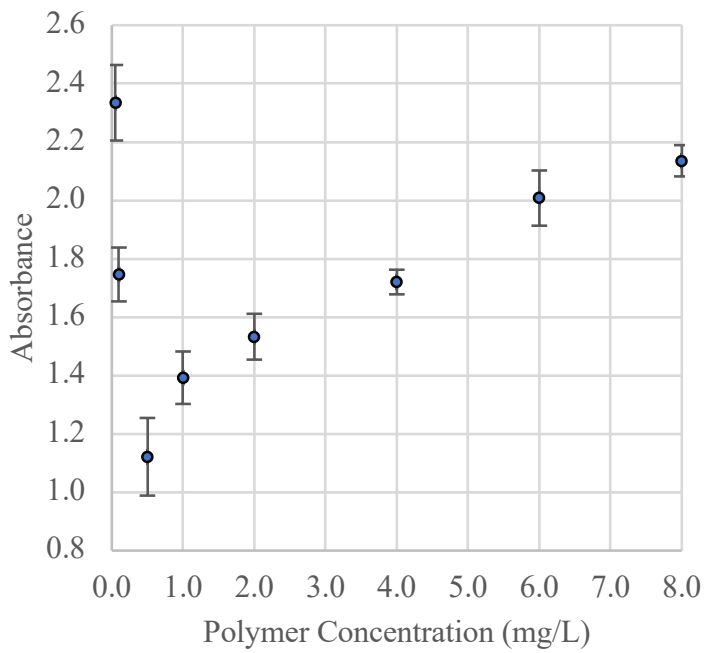

Figure 54: Linear correlation of Magnafloc LT27AG absorbance at 190nm in high turbidity (9000 NTU) Rideau River water dosed with Actiflo $\Theta$ enhanced coagulation process chemicals using a (a) desktop spectrophotometer and a (b) inline spectrophotometer for determination of the sample's optimum polymer dose

The linear correlations depicted in Figures 52, 53 and 54 demonstrate some very interesting and promising results for the use of UV-vis spectroscopy as a tool for the optimization of polymer dose in drinking water treatment applications. The results of the highly turbid water samples dosed with polymer, as seen in Figure 52, demonstrate that on both the desktop and in-line spectrophotometers, the lowest absorbance value, 2.220 A.U. on the desktop unit and 1.240 A.U. on the in-line unit, corresponds to the sample with a $2.0 \mathrm{mg} / \mathrm{L}$ concentration of Magnafloc polymer. When comparing this value to that of the predetermined optimum dose done using a settling test, the value of $2.0 \mathrm{mg} / \mathrm{L}$ remains 
consistent. When the polymer concentration was lower than $2.0 \mathrm{mg} / \mathrm{L}$, or under-dosed, the sample dosed with a $0.10 \mathrm{mg} / \mathrm{L}$ concentration of polymer resulted in an increase in absorbance values on both spectrophotometers, 2.940 A.U. for the desktop unit and $1.34 \mathrm{mg} / \mathrm{L}$ on the Real tech unit. As the polymer dose concentration is further increased from $0.1 \mathrm{mg} / \mathrm{L}$ towards $2.0 \mathrm{mg} / \mathrm{L}$, a steady decrease in absorbance is observed on both the desktop and in-line spectrophotometers. Beyond $2.0 \mathrm{mg} / \mathrm{L}$, or when the polymer is overdosed, again a steady increase in absorbance is observed until the maximum polymer dose of $5.0 \mathrm{mg} / \mathrm{L}$ is reached, resulting in absorbance value increases to 2.470 A.U. on the desktop unit and 1.590 A.U. on the in-line unit. This is further observed in Figure 46, which depicts the turbidity data for the supernatant of the same sample set. The turbidity results also show a minimum value when the sample was dosed with $2.0 \mathrm{mg} / \mathrm{L}$ of polymer, indicating that for the highly turbid water sample a polymer dose of $2.0 \mathrm{mg} / \mathrm{L}$ resulted in the clearest supernatant after settling, and was thus dosed optimally with polymer. In addition, the turbidity results also show very similar trends to the absorbance results in the under-dosed range, indicating that as polymer dosage is increased towards an optimum concentration, the turbidity decreases steadily as well. In the overdosed range, however, the turbidity results neither increase nor decrease with increasing polymer concentration and instead remain fairly constant.

When observing Figures 53 and 54, for the low turbidity water sample and the high turbidity water sample dosed with Actiflo enhanced coagulation process chemicals, respectively, very similar results are demonstrated. In Figure 53, both spectrophotometers indicated that the lowest absorbance value, 2.170 A.U. using the desktop unit and 1.060 
A.U. using the in-line unit, corresponded to the sample with a $0.10 \mathrm{mg} / \mathrm{L}$ polymer concentration in Rideau River water with a low turbidity. This value is in accordance with the optimum dose predetermined using the preliminary settling tests, as well as within the range of the turbidity measurements for the supernatant of the same sample set presented in Figure 47. The turbidity measurements indicated that the lowest turbidity (107 NTU) corresponded to the sample dosed with $0.05 \mathrm{mg} / \mathrm{L}$ of polymer, however the sample dosed with $0.10 \mathrm{mg} / \mathrm{L}$ of polymer was only marginally more turbid (108 NTU). Moreover, Figure 54 demonstrates that both spectrophotometers reported a minimum supernatant absorbance value, 2.540 A.U. for the desktop unit and 1.120 A.U. for the in-line unit, corresponding to the sample with a $0.50 \mathrm{mg} / \mathrm{L}$ polymer concentration. This value is in accordance with the optimum dose predetermined using the preliminary settling tests, as well as within the range of the turbidity measurements for the supernatant of the same sample set presented in Figure 48. The turbidity measurements indicated that the lowest turbidity (373 NTU) corresponded to the sample dosed with $1.0 \mathrm{mg} / \mathrm{L}$ of polymer, however the sample dosed with $0.5 \mathrm{mg} / \mathrm{L}$ of polymer was only marginally more turbid (385 NTU). Both Figures 53 and 54 also followed the same curve shape trends, showing a decrease in absorbance in the under-dose range until the optimum polymer dose is reached, at which point the overdose of polymer corresponds to an increase in absorbance.

The absorbance results presented in Figures 52, 53 and 54 and their accordance with the turbidity results presented in Figures 46, 47 and 48 demonstrate that the use of UV-vis spectroscopy as a method for the determination of optimum polymer dose is very 
promising. These results also indicate that the method can be replicated in water matrices of both high and low turbidity, with and without the addition of process chemicals.

\subsubsection{Total particle concentration}

In order to further explore the trend between optimum polymer dose and minimum turbidity as well as minimum absorbance, particle counts were also measured from each sample of supernatant for all three water matrices using a Dynamic Particle Analyzer (BP4100-HEV model) manufactured by BrightWell Technologies INC (Ottawa, ON). It was hypothesized that the optimum polymer dose should remove the greatest number of particles from the water sample, and therefore the supernatant should in turn contain a minimum number of particles at this polymer concentration. Figure 55 below represents the relationship between polymer concentration and particle concentration in the supernatant of a high turbidity (9172 NTU) Rideau River water sample.

Total Particle Concentration vs. Polymer Dose

Magnafloc in High Turbidity Sample (9172 NTU)

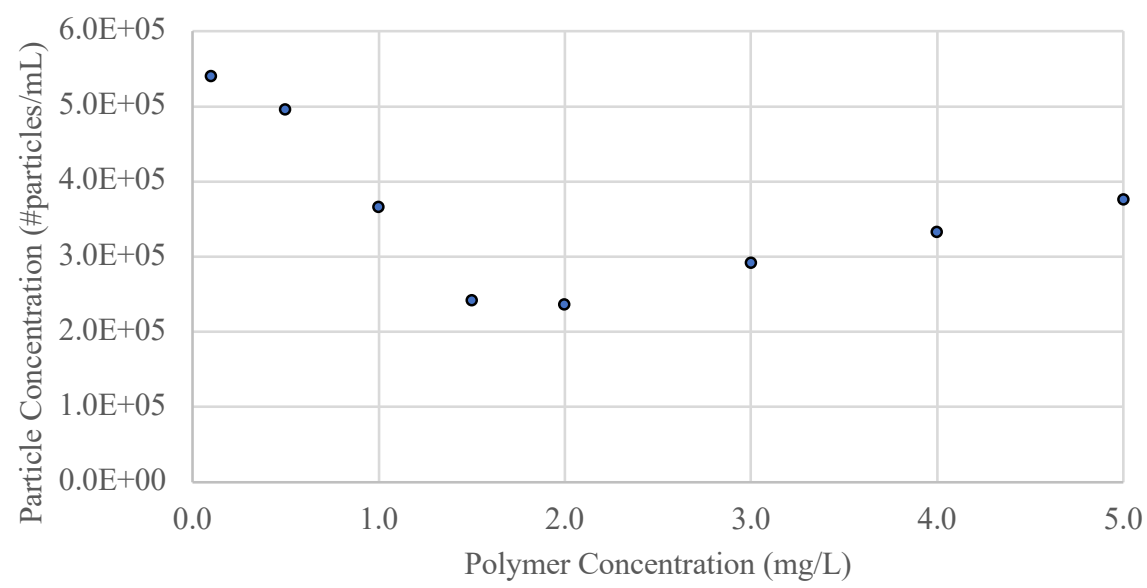

Figure 55: Particle counts corresponding to Magnafloc LT27AG polymer concentrations within the optimum dose range in the supernatant of a high turbidity (9172 NTU) Rideau River water sample 
Figures 56 and 57 below represent the relationship between polymer concentration and particle concentration in the supernatant of a low turbidity (260 NTU) Rideau River water sample and a high turbidity (9000 NTU) Rideau River water sample dosed with Actiflo ${ }^{\circledR}$ enhanced coagulation process chemicals, respectively.

Total Particle Concentration vs. Polymer Dose

Magnafloc in Low Turbidity Sample (290 NTU)

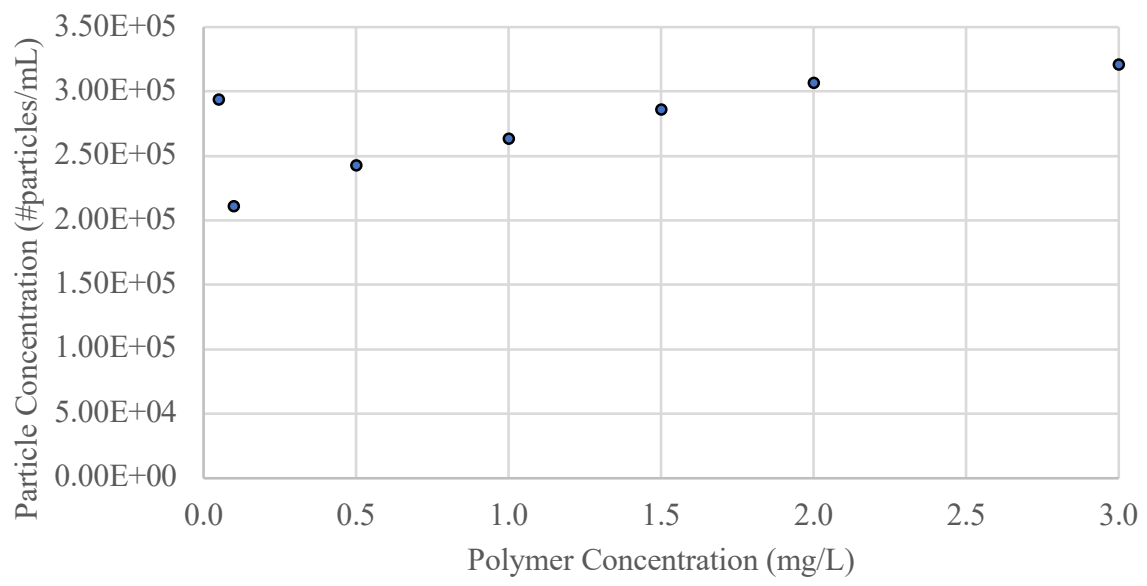

Figure 56: Particle counts corresponding to Magnafloc LT27AG polymer concentrations within the optimum dose range in the supernatant of a low turbidity (260 NTU) Rideau River water sample

Total Particle Concentration vs. Polymer Dose Magnafloc in High Turbidity Sample (9000 NTU) with Actiflo

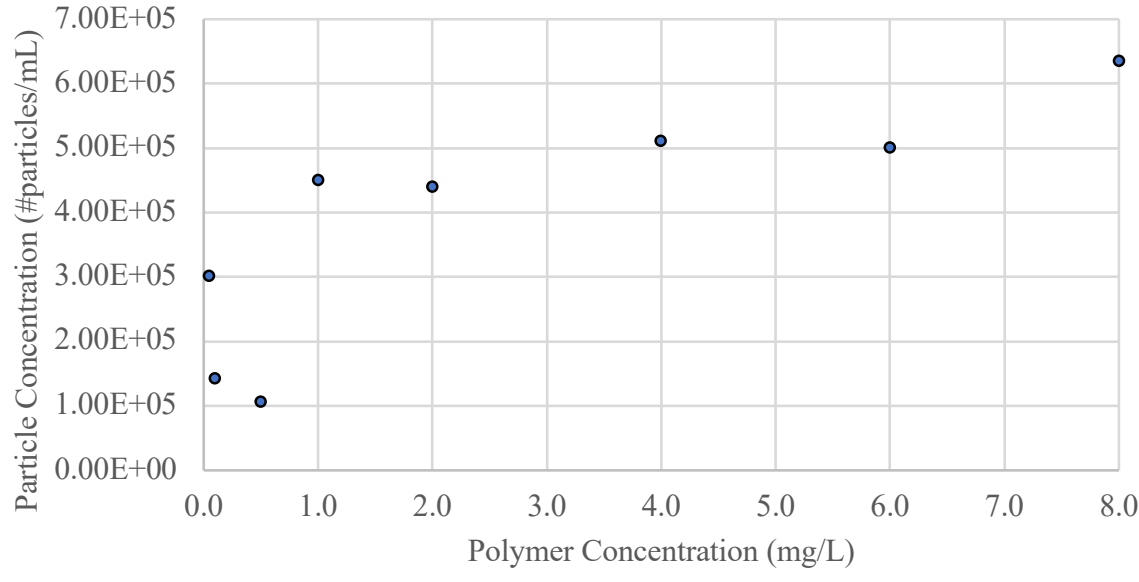

Figure 57: Particle counts corresponding to Magnafloc LT27AG polymer concentrations within the optimum dose range in the supernatant of a high turbidity (9000 NTU) Rideau River water sample dosed with Actiflo $®$ enhanced coagulation process chemicals 
Figure 55 illustrates that the lowest particle concentration $\left(2.36 \times 10^{5}\right.$ \#particles $\left./ \mathrm{mL}\right)$ coincides with the sample dosed with a polymer concentration of $2.0 \mathrm{mg} / \mathrm{L}$. This polymer concentration is in accordance with the optimum polymer dose determined through the preliminary settling test results depicted in Table 13, the turbidity results depicted in Figure 46, and the absorbance results depicted in Figure 52. The trends observed in all other results for this high turbidity water sample are once again observed in the particle count results, demonstrating an increase in total particle concentration $\left(5.40 \times 10^{5} \mathrm{\# particles} / \mathrm{mL}\right)$ in the under-dose range which then incrementally decreases as the polymer dose is increased towards the optimum concentration of $2.0 \mathrm{mg} / \mathrm{L}$. Beyond this concentration, as the polymer dose is increased, the total particle concentration then increases incrementally to a value of $3.76 \times 10^{5}$ \#particles/mL corresponding to the maximum polymer dose of $5.0 \mathrm{mg} / \mathrm{L}$.

Figures 56 shows very similar trends in the low turbidity water sample, illustrating that the lowest total particle concentration $\left(2.11 \times 10^{5}\right.$ \#particles $\left./ \mathrm{mL}\right)$ corresponds to a polymer concentration of $0.10 \mathrm{mg} / \mathrm{L}$. This polymer concentration is in accordance with the optimum polymer dose determined using the UV-vis spectroscopy absorbance method depicted in Figure 53. The preliminary settling test, depicted in Table 14, and the turbidity results, depicted in Figure 47, suggested the optimum polymer dose be slightly lower at a concentration of $0.05 \mathrm{mg} / \mathrm{L}$. These preliminary results were likely not as sensitive as the absorbance and particle count results, as there was very marginal difference in turbidity between $0.10 \mathrm{mg} / \mathrm{L}$ and $0.05 \mathrm{mg} / \mathrm{L}$ concentrations of polymer; whereas there is a more noticeable distinction between the difference in absorbance (1.180 A.U. and 1.060 A.U., 
respectively, using the in-line unit) and the difference in particle concentration $\left(2.94 \times 10^{5}\right.$ $\#$ particles $/ \mathrm{mL}$ and $2.11 \times 10^{5}$ \#particles $/ \mathrm{mL}$, respectively) between the two concentrations.

Again, Figure 57 demonstrates very similar trends in the high turbidity water sample dosed with Actiflo ${ }^{\circledR}$ process chemicals, with results showing a minimum total particle concentration corresponding to a polymer concentration of $0.50 \mathrm{mg} / \mathrm{L}$. This polymer concentration is in accordance with the optimum polymer dose determined using the UVvis spectroscopy absorbance method depicted in Figure 54. The preliminary settling test, depicted in Table 15, and the turbidity results, depicted in Figure 48, suggested the optimum polymer dose be slightly higher at a concentration of $1.0 \mathrm{mg} / \mathrm{L}$. Again, this variation is likely due to the fact that UV-vis spectroscopy and particle counting offer more sensitive results as there was very marginal difference in turbidity between $0.50 \mathrm{mg} / \mathrm{L}$ and $1.0 \mathrm{mg} / \mathrm{L}$ concentrations of polymer; whereas there is a more noticeable distinction between the difference in absorbance (1.120 A.U. and 1.390 A.U., respectively, using the in-line unit) and the difference in particle concentration $\left(1.07 \times 10^{5} \#\right.$ particles $/ \mathrm{mL}$ and $4.51 \times 10^{5}$ \#particles/mL, respectively) between the two concentrations.

The fact that the particle count results between all three water sample matrices coincide with the absorbance results infers that UV-vis spectroscopy has the viability to be used as a tool for the optimization of polymer dose for Magnafloc LT27AG. The preliminary settling test and turbidity results are also in accordance with these results, furthering this inference. In addition, the ability of this method to succeed in a variety of different water matrices demonstrates replicability. 


\subsubsection{Hydrex 3661 Polymer}

\subsubsection{Settling tests}

Hydrex 3661 is a cationic polyacrylamide polymer with a high molecular weight. This polymer was used to replicate the same experimental setup as was done for the Magnafloc LT27AG polymer, with the aim of testing the replicability of UV-vis spectroscopy as a method for determining the optimum polymer dose in both a high and low turbidity water sample using a polymer of opposing charge. Using the same method as was done using the Magnafloc LT27AG polymer, a preliminary settling test was performed on both high (4426 NTU) and low (546 NTU) turbidity water matrices in order to determine the optimum polymer dose range based on apparent clarity of the supernatant and the fastest settling time. Dissimilar to the tests performed using Magnafloc LT27AG, the experiments carried out using Hydrex 3661 were not performed in water matrices dosed with Actiflo® enhanced coagulation treatment chemicals due to the fact that this process specifies the use of Magnafloc LT27AG polymer only. Tables 16 and 17 below represent the results of the settling tests for the high turbidity Rideau River water and the low turbidity Rideau River water, respectively. 
Table 16: Settling test data for high turbidity (4426 NTU) Rideau River Water dosed with Hydrex 3661 polymer

\begin{tabular}{ccc}
\hline Polymer Concentration $\mathbf{( m g / L )}$ & Interface $(\mathbf{m m})$ & Supernatant Turbidity (NTU) \\
\hline 0.00 & N/A & 4426 \\
\hline 3.00 & 2.0 & 171 \\
\hline 4.00 & 4.0 & 122 \\
\hline 5.00 & 3.0 & 106 \\
\hline 6.00 & 4.0 & 92 \\
\hline 7.00 & 4.0 & 126 \\
\hline 8.00 & 4.0 & 93 \\
\hline 10.00 & 5.0 & 118 \\
\hline 12.00 & 5.0 & 129 \\
\hline Optimum Settling Time & 210 seconds at $8.00 \mathrm{mg} / \mathrm{L}$ polymer dose
\end{tabular}

Table 17: Settling test data for low turbidity (546 NTU) Rideau River Water dosed with Hydrex 3661 polymer

\begin{tabular}{ccc}
\hline Polymer Concentration $\mathbf{( m g} / \mathbf{L})$ & Interface $(\mathbf{m m})$ & Supernatant Turbidity (NTU) \\
\hline 0.00 & N/A & 546 \\
\hline 0.025 & $\sim 1.0$ & 395 \\
\hline 0.05 & $\sim 1.0$ & 350 \\
\hline 0.10 & $\sim 1.0$ & 325 \\
\hline 0.50 & $\sim 1.0$ & 231 \\
\hline 1.00 & $\sim 1.0$ & 155 \\
\hline 2.00 & 2.0 & 113 \\
\hline 4.00 & 2.0 & 98 \\
\hline 6.00 & $\sim 1.0$ & 107 \\
\hline 8.00 & $\sim 1.0$ & 90 \\
\hline 10.00 & $\sim 1.0$ & 108 \\
\hline Optimum Settling Time & 262 seconds at $2.00 \mathrm{mg} / \mathrm{L}$ polymer dose
\end{tabular}

From these preliminary settling test results, the optimum dose of Hydrex 3661 polymer was determined to be $8.0 \mathrm{mg} / \mathrm{L}$ for the high turbidity Rideau River and $2.0 \mathrm{mg} / \mathrm{L}$ for the low turbidity Rideau River water. These results were based on apparent clarity of the supernatant and the fastest settling time. The interface post-settling was quite variable in both the high and low turbidity water samples, and not much of a trend is observed. 


\subsubsection{Turbidity measurements}

As was done for samples dosed with Magnafloc LT27AG polymer, the supernatant of the settled samples dosed with Hydrex 3661 polymer was collected and used for turbidity measurements, absorbance measurements as well as particle count measurements. In order to better visualize the relationship between turbidity and polymer dosage, the data was plotted in scatter plot form. Figures 58 and 59 below represent the turbidity data in relation to polymer concentration for the supernatant of the high turbidity (4426 NTU) and the low turbidity (546 NTU) Rideau River water samples dosed with polymer both over and underdosing the preliminarily determined optimum dosage range, respectively.

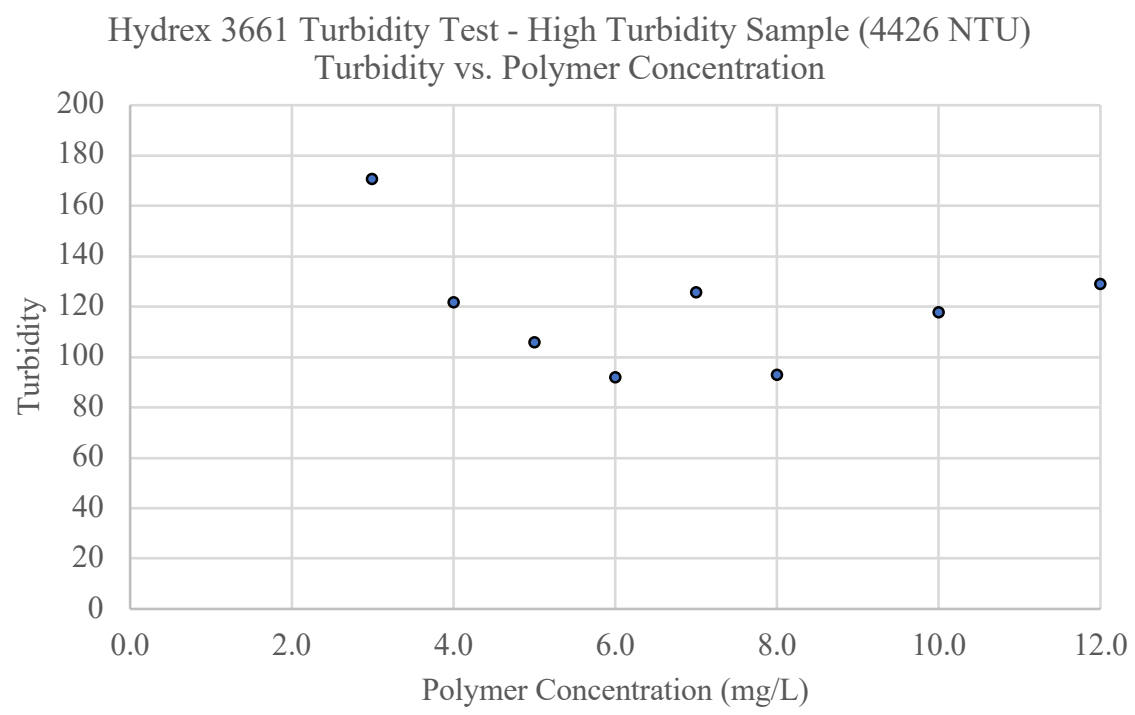

Figure 58: Turbidity values corresponding to Hydrex 3661 polymer concentrations within the optimum dose range in a high turbidity (4426 NTU) Rideau River water sample supernatant 


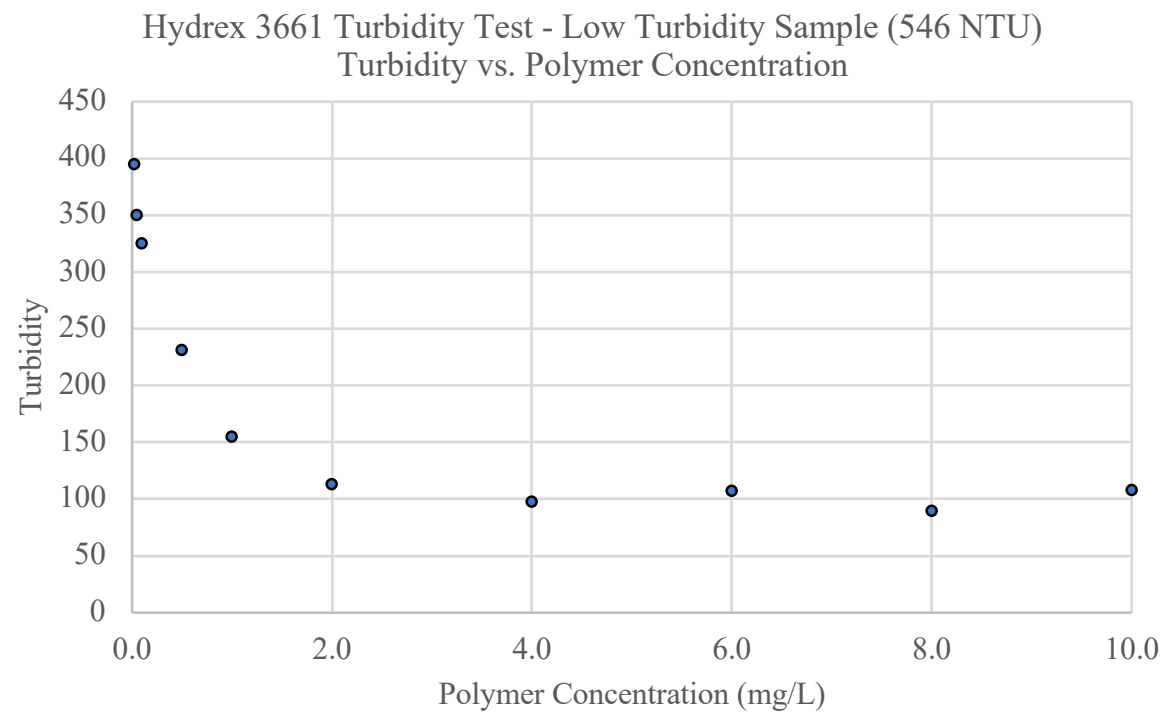

Figure 59: Turbidity values corresponding to Hydrex 3661 polymer concentrations within the optimum dose range in a low turbidity (546 NTU) Rideau River water sample supernatant

Figure 58, representing the relationship between turbidity and polymer concentration in the supernatant of a high turbidity (4426 NTU) Rideau River water sample, reports that the minimum turbidity (92 NTU) corresponds to the sample dosed with $6.0 \mathrm{mg} / \mathrm{L}$ of polymer. This polymer concentration differs slightly from the preliminary value determined using the settling test, which determined the fastest settling time to occur at a polymer dose of $8.0 \mathrm{mg} / \mathrm{L}$. However, the turbidity between the $6.0 \mathrm{mg} / \mathrm{L}$ sample (92 NTU) was only marginally less than that of the $8.0 \mathrm{mg} / \mathrm{L}$ sample ( $93 \mathrm{NTU}$ ), implying that the turbidimeter does not possess the sensitivity to detect a substantial difference between these polymer dosages. As was expected, an under-dose of polymer resulted in an increase in turbidity. As polymer dosage was increased toward the optimum value, the turbidity decreased accordingly. At a polymer dose of $7.0 \mathrm{mg} / \mathrm{L}$, one incremental dose higher than the presumed optimum dose, a spike in turbidity was observed (126 NTU). Beyond $7.0 \mathrm{mg} / \mathrm{L}$, in the overdose range, the expected trend was observed and only a slight increase in turbidity was noted once again. This spike in turbidity at a polymer concentration of $7.0 \mathrm{mg} / \mathrm{L}$ may once 
again be attributed to the fact that the bulk sample, assumed to be homogeneous postmixing, may have not settled for as long a period as the other samples in the set. This would lead to a potential higher initial turbidity of this specific sample, thus resulting in a higher turbidity post-settling test. Regardless of this spike, the remaining samples of varying polymer concentration tend to the expected trend.

Figure 59, representing the relationship between turbidity and polymer concentration in the supernatant of a low turbidity (546 NTU) Rideau River water sample, reports that the minimum turbidity (90 NTU) corresponds to the sample dosed with $8.0 \mathrm{mg} / \mathrm{L}$ of Hydrex 3661 polymer. This concentration value differs from the predetermined settling test estimates, which suggested the optimum polymer dose should be in the range of $2.0 \mathrm{mg} / \mathrm{L}$ for this particular water matrix. In the under-dose range, the turbidity once again follows the expected trend of decreasing with increasing polymer concentration. However, at $4.0 \mathrm{mg} / \mathrm{L}$, the turbidity rests at a value of $98 \mathrm{NTU}$, increasing only slightly to $107 \mathrm{NTU}$ (at a $6.0 \mathrm{mg} / \mathrm{L}$ polymer concentration) before reaching the minimum value of $90 \mathrm{NTU}$. In the overdosed range, the turbidity once again increases marginally with increasing polymer dose. Between $4.0 \mathrm{mg} / \mathrm{L}$ and $10.0 \mathrm{mg} / \mathrm{L}$, the variance in turbidity veers only slightly and the exact optimum dose is suggestive. These results again indicate that in water matrices of lower turbidity, determining the optimum polymer dose using turbidity measurements alone may not be a viable method for accurate results in drinking water treatment applications. 


\subsubsection{UV-vis absorbance spectra}

As was done for the samples prepared using Magnafloc LT27AG, the viability of using UV-vis spectrophotometry as a method of determining optimum polymer dose for cationic polymers in drinking water treatment applications was tested. This was again done by comparing the preliminary optimum dose results of the settling test and turbidity measurements to absorbance results produced through UV-vis spectroscopy using both a desktop and in-line spectrophotometer. The supernatant of the samples prepared for the settling tests and turbidity measurements was reserved and used for the UV-vis spectroscopy absorbance tests as well. Figures $60 \mathrm{a}$ and $60 \mathrm{~b}$ below represent the absorbance spectra of the supernatant of high turbidity (4426 NTU) Rideau River water samples dosed with Hydrex 3661 polymer at concentrations both overdosing and under-dosing the optimum polymer dose range using a desktop and in-line spectrophotometer, respectively.

(a) desktop spectrophotometer

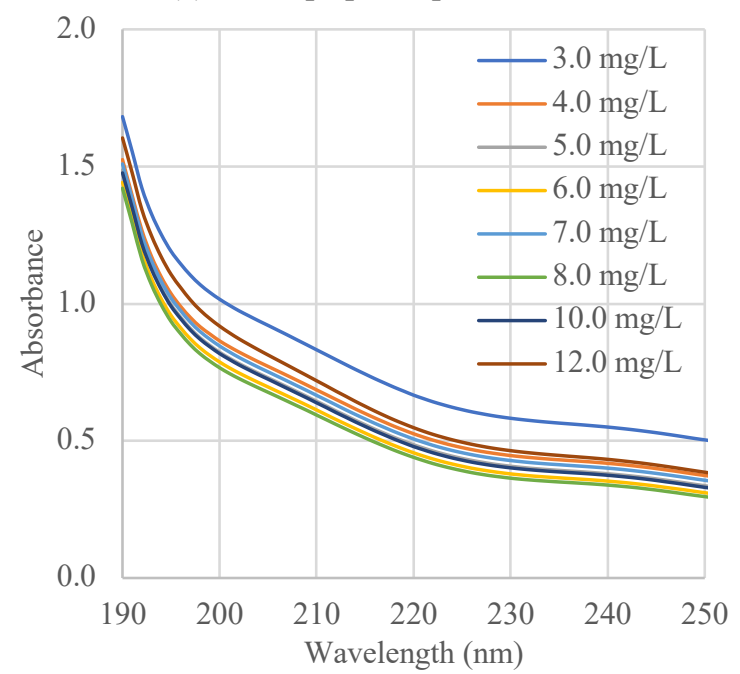

(b) in-line spectrophotometer

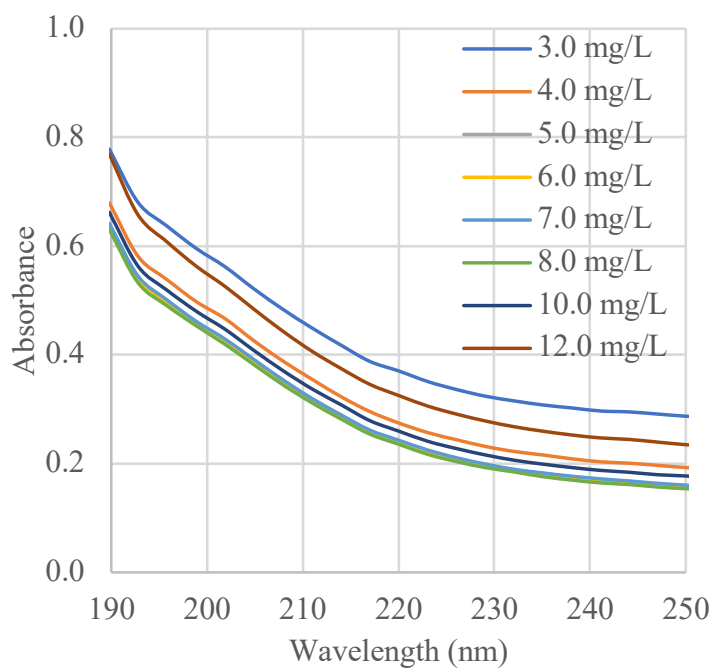

Figure 60: UV-vis absorbance spectra of Hydrex 3661 polymer in a sample of high turbidity (4426 NTU) Rideau River water using a (a) desktop spectrophotometer and a (b) in-line spectrophotometer for identifying the samples optimum polymer dose 
Figures $61 \mathrm{a}$ and $61 \mathrm{~b}$ below represent the absorbance spectra of the supernatant of low turbidity (546 NTU) Rideau River water samples dosed with Hydrex 3661 polymer at concentrations both overdosing and under-dosing the optimum polymer dose range using a desktop and in-line spectrophotometer, respectively.

(a) desktop spectrophotometer

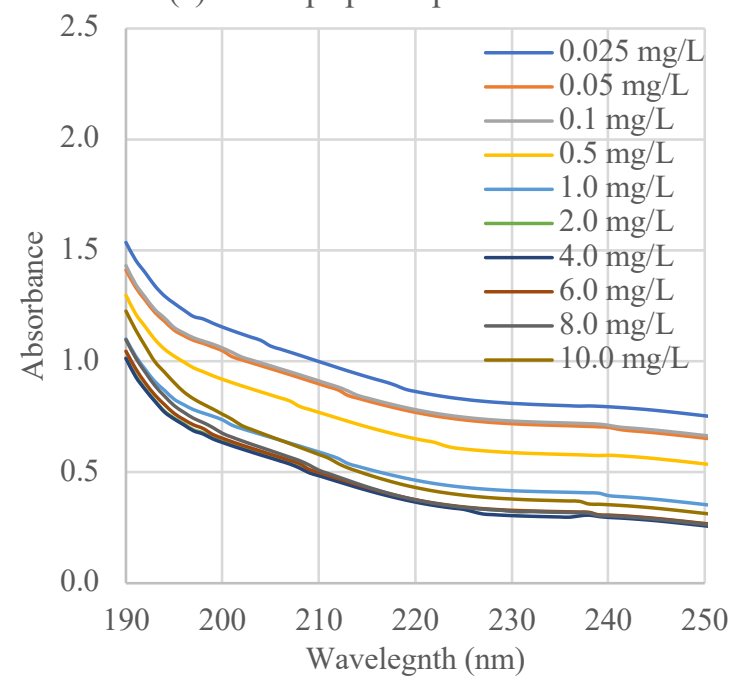

(b) in-line spectrophotometer

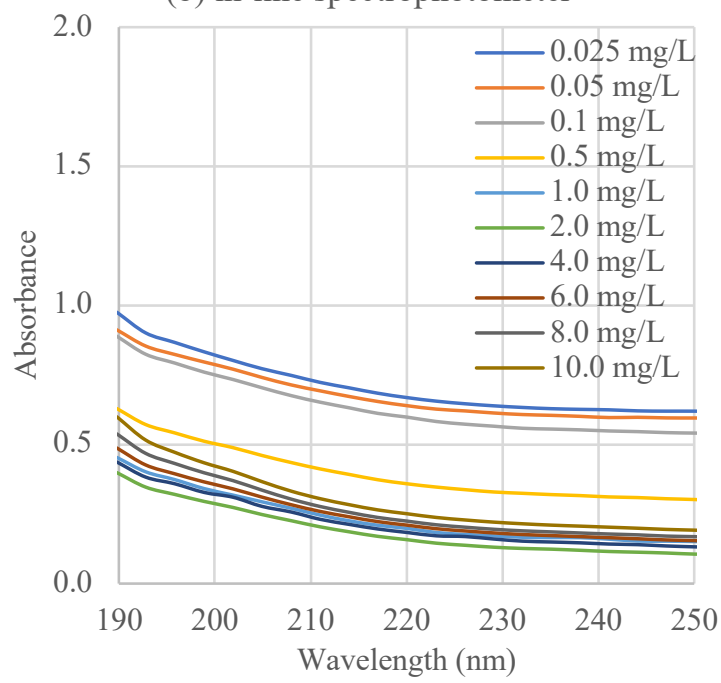

Figure 61: UV-vis absorbance spectra of Hydrex 3661 polymer in a sample of low turbidity (546 NTU) Rideau River water using a (a) desktop spectrophotometer and a (b) in-line spectrophotometer for identifying the samples optimum polymer dose

As is observed in Figures 60 and 61, both the high and low turbidity Rideau River water samples resulted in absorbance spectra with the expected shape and peak absorbance at $190 \mathrm{~nm}$ using both the desktop and in-line spectrophotometers. The absorbance spectra curves of each different concentration are again more sparsely distributed than those in experimental phases one, two and three. This is expected, seeing that the polymer is overdosed and under-dosed relative to the optimum dose rather than dosed in equal increments as has been done for all previous experimental phases. This, in-turn, results in curves that increase un-uniformly between polymer concentrations. 


\subsubsection{Linear correlations}

As for all other absorbance data gathered during the previous experimental phases, linear correlations of peak absorbance data versus polymer concentration from Figures 58 and 59 were plotted in order to further analyze the absorbance-polymer concentration relationship and determine whether or not UV-vis spectroscopy is a method capable of observing the optimum polymer dose in each different water sample. Figures $62 \mathrm{a}$ and $62 \mathrm{~b}$ below represent the linear correlations of high turbidity (4426 NTU) Rideau River water samples overdosed and under-dosed with Hydrex 3661 polymer relative to the polymers predetermined optimum dosage range using both a desktop and in-line spectrophotometer, respectively.

(a) desktop spectrophotometer

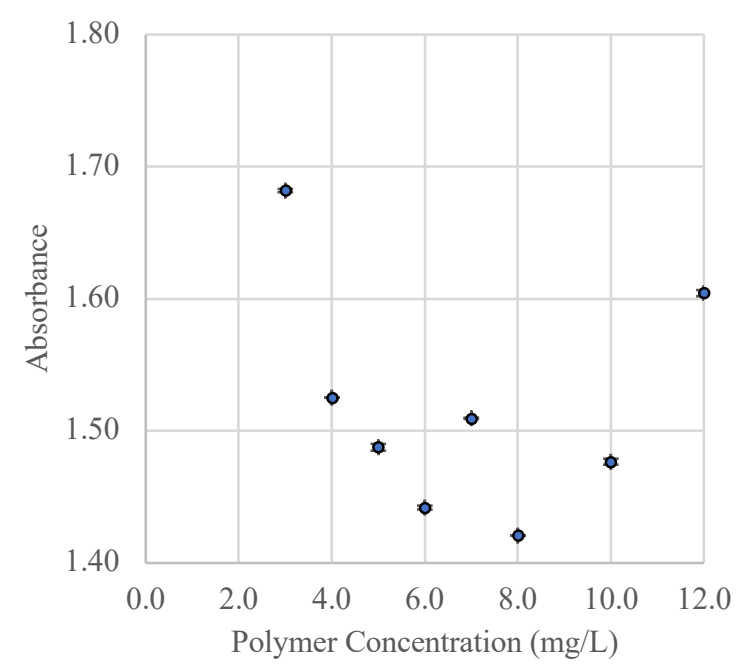

(b) in-line spectrophotometer

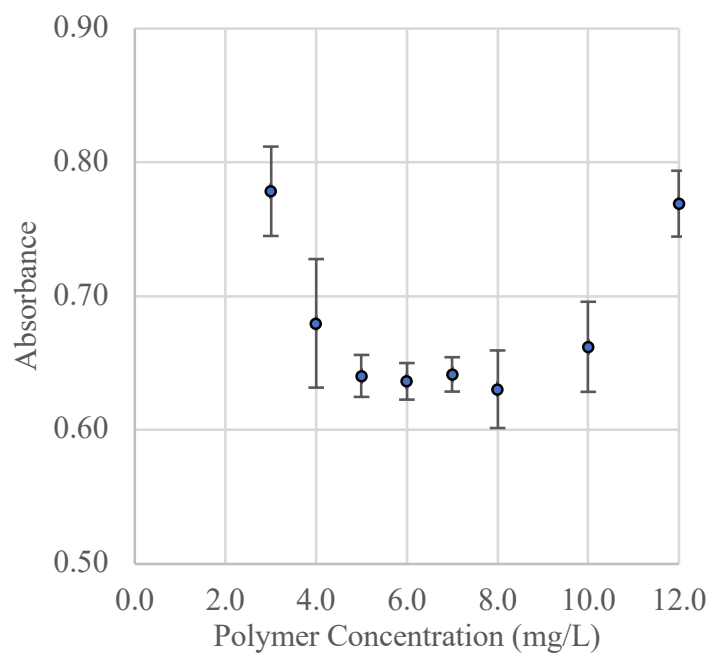

Figure 62: Linear correlation of Hydrex 3661 polymer absorbance at 190nm in high turbidity (4426 NTU) Rideau River water using a (a) desktop spectrophotometer and a (b) in-line spectrophotometer for determination of the sample's optimum polymer dose 
Figures $63 \mathrm{a}$ and $63 \mathrm{~b}$ below represent the linear correlations of low turbidity (546 NTU)

Rideau River water samples overdosed and under-dosed with Hydrex 3661 polymer relative to the polymers predetermined optimum dosage range using both a desktop and inline spectrophotometer, respectively.

(a) desktop spectrophotometer

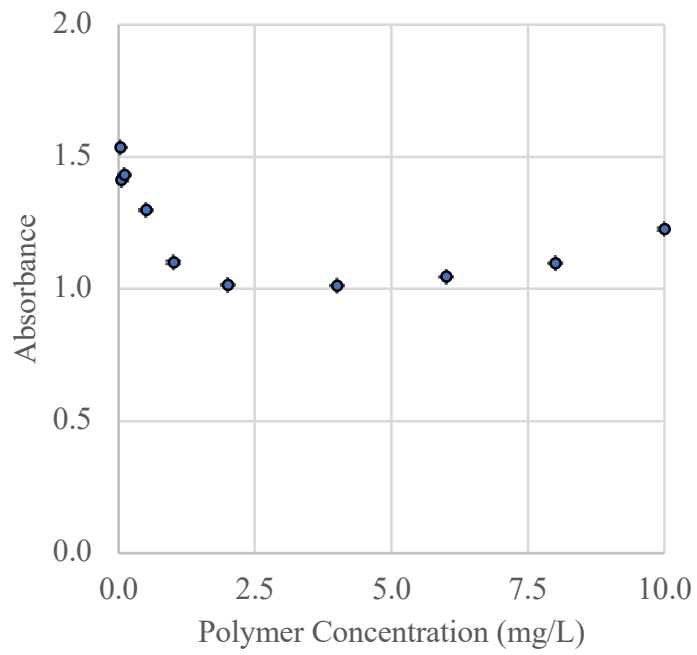

(b) in-line spectrophotometer

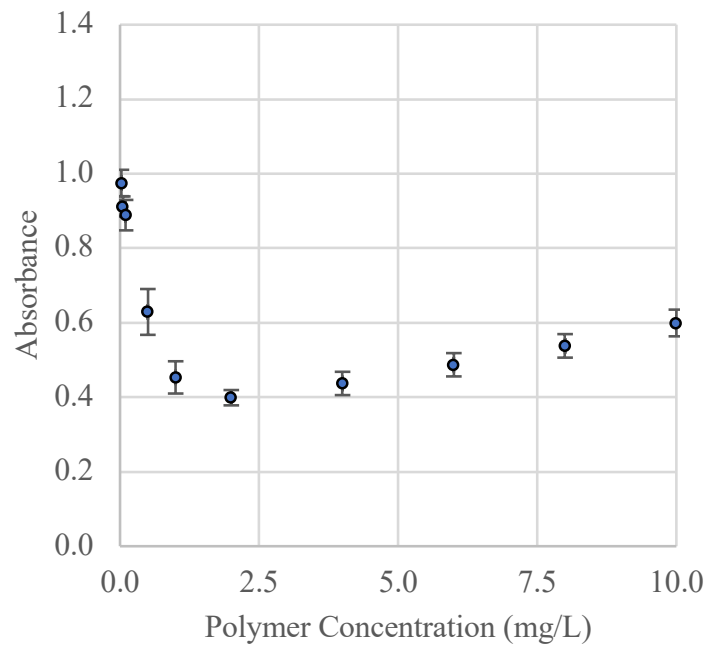

Figure 63: Linear correlation of Hydrex 3661polymer absorbance at 190nm in low turbidity (546 NTU) Rideau River water using a (a) desktop spectrophotometer and a (b) in-line spectrophotometer for determination of the sample's optimum polymer dose

The linear relationship between polymer concentration and absorbance at $190 \mathrm{~nm}$ of the highly turbid water sample (Figure 62) demonstrate very similar trends to those observed in a high turbidity sample dosed with Magnafloc LT27AG (Figure 52). Again, in the underdose range, the absorbance decreases with increasing polymer concentration until it reaches a minimum value at $8.0 \mathrm{mg} / \mathrm{L}$ on both spectrophotometers; 1.420 A.U. on the desktop unit and 0.630 A.U. on the in-line unit. This value is in accordance with the value estimated through the preliminary settling tests and turbidity results for this water sample. Beyond this concentration, as polymer dose increases, the absorbance again demonstrates an 
increase in absorbance with increasing polymer concentration. This increase in absorbance is notably more prominent than the increase in turbidity for the same supernatant samples, observed in Figure 58. This is likely due to the fact that beyond the optimum dosage, the polymer is no longer effective and therefore remains suspended in the solution as residual rather than absorbing onto particulates present in the sample and settling out. UV-vis spectroscopy is able to detect this residual polymer increase with increasing dose, whereas the turbidity measurements are not able to detect this. Another notable difference are the results between spectrophotometers, the desktop unit spiking in absorbance at the $7.0 \mathrm{mg} / \mathrm{L}$ sample (Figure 62a) and the in-line unit demonstrating more of the expected relationship (Figure 62b), implying the in-line unit has a higher detection sensitivity and is able to detect very slight changes in polymer dosage at a wavelength of $190 \mathrm{~nm}$.

The linear relationship between polymer concentration and absorbance in a low turbidity water sample (Figure 63) again shows very similar trends as observed with previous samples used for this experiment. The minimum absorbance value at $190 \mathrm{~nm}$ using the desktop unit measured 1.013 A.U. and is observed at a concentration of $4.0 \mathrm{mg} / \mathrm{L}$ of polymer. This value differs slightly from that read using the in-line unit, which detected a minimum absorbance value ( 0.399 A.U.) corresponding to a polymer concentration of $2.0 \mathrm{mg} / \mathrm{L}$. Comparing the absorbance values at $190 \mathrm{~nm}$ between $2.0 \mathrm{mg} / \mathrm{L}$ and $4.0 \mathrm{mg} / \mathrm{L}$ read using the desktop unit (Figure 63a) there is a very marginal difference of 0.003 A.U. Alternatively, when comparing these same values read using the in-line unit (Figure 63b) there is a more notable difference in absorbance of 0.038 A.U. between the $2.0 \mathrm{mg} / \mathrm{L}$ and 4.0mg/L samples. Again, the ability of the in-line unit to detect a larger variance in 
absorbance between samples of varying polymer concentration infers that this spectrophotometer is a more sensitive tool for the detection of residual polymer than the desktop unit. Using this logic, the optimum polymer dosage should therefore correspond to $2.0 \mathrm{mg} / \mathrm{L}$, as reported by the in-line spectrophotometer. In the under-dose and overdose ranges, both spectrophotometers demonstrate the expected trends as observed in previous samples. The optimum dosage value of $2.0 \mathrm{mg} / \mathrm{L}$ determined using the $\mathrm{UV}$-vis spectroscopy method differs from the optimum dose value of $8.0 \mathrm{mg} / \mathrm{L}$ determined through the preliminary turbidity measurements; however, it is in accordance with the value of $2.0 \mathrm{mg} / \mathrm{L}$ determined using the preliminary settling tests and settling time. As was previously noted, the turbidity test method of determining the optimum polymer dose lacks in sensitivity in the overdose range, whereas the UV-vis absorbance measurement method is able to detect residual polymer in the overdose range and indicate its presence in solution through an increase in absorbance. This results in a more obvious minimum absorbance reading as opposed to the more ambiguous minimum turbidity reading, which is likely why the absorbance measurements are in accordance with the settling test estimates for the optimum dosage of Hydrex 3661 in the low turbidity water sample.

The results of the linear relationship between polymer concentration and absorbance presented in Figures 62 and 63; and their accordance with the turbidity results presented in Figures 58 and 59 demonstrate that the use of UV-vis spectroscopy as a method for the determination of optimum polymer dose for cationic polymers is very promising. These results also indicate that the method can be replicated not only in water matrices of both high and low turbidity, but also using polymers of variable charge. 


\subsubsection{Total particle concentration}

As was done for the samples prepared with Magnafloc LT27AG polymer, the trend between optimum polymer dose and minimum turbidity as well as minimum absorbance was compared to the total particle concentration of each sample of supernatant for both water matrices using a Dynamic Particle Analyzer (BP-4100-HEV model) manufactured by BrightWell Technologies INC (Ottawa, ON). It was hypothesized that the optimum polymer dose for the cationic polymer should again remove the greatest number of particles from the water sample, and therefore the supernatant should in turn contain a minimum number of particles at this polymer concentration. Figures 64 and 65 below represent the relationship between polymer concentration and particle concentration in the supernatant of a high turbidity (4426 NTU) and low turbidity (546 NTU) Rideau River water sample dosed with Hydrex 3661, respectively.

Total Particle Concentration vs. Polymer Dose Hydrex 3661 in High Turbidity Sample (4426 NTU)

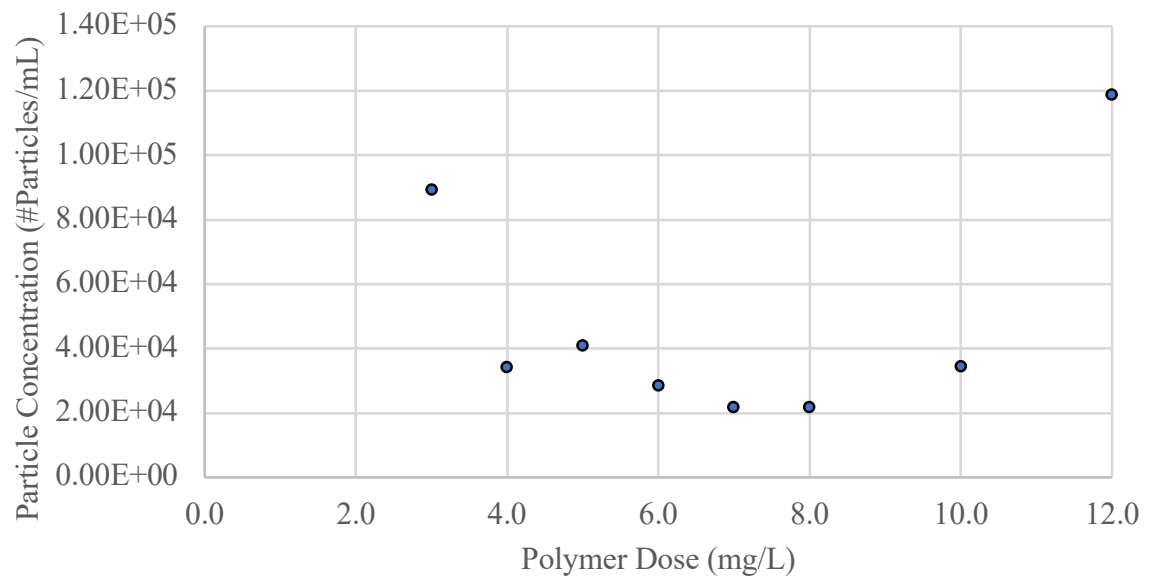

Figure 64: Particle counts corresponding to Hydrex 3661 polymer concentrations within the optimum dose range in a high turbidity (4426 NTU) Rideau River water sample supernatant 
Total Particle Concentration vs. Polymer Dose

Hydrex 3661 in Low Turbidity Sample (546 NTU)

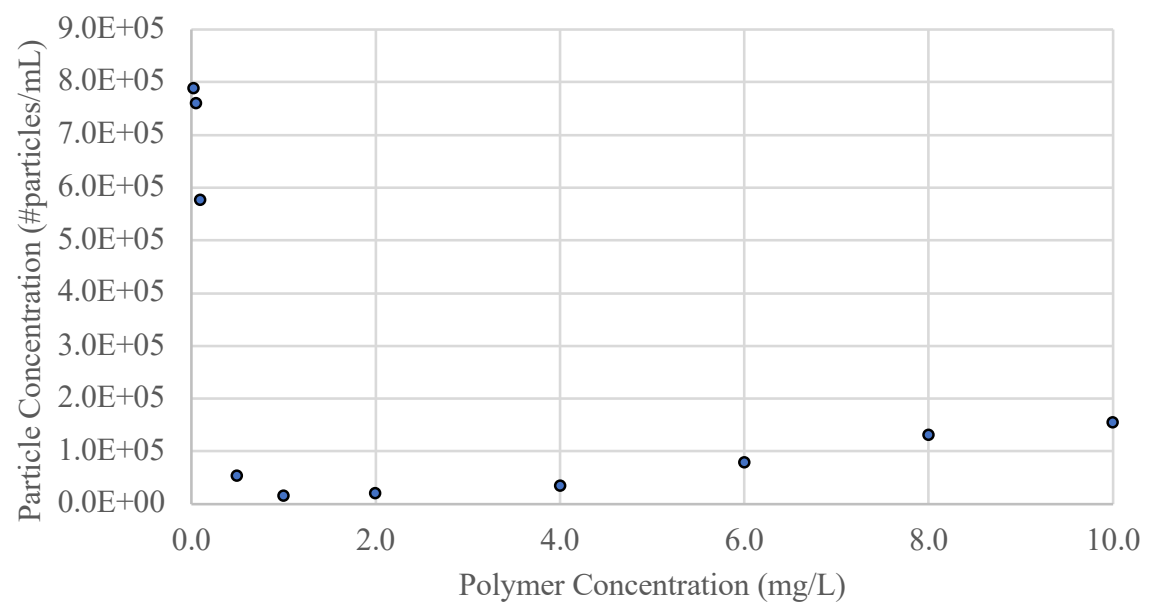

Figure 65: Particle counts corresponding to Hydrex 3661 polymer concentrations within the optimum dose range in a low turbidity (546 NTU) Rideau River water sample supernatant

Figure 64 illustrates that the lowest particle concentration $\left(2.18 \times 10^{4}\right.$ \#particles $\left./ \mathrm{mL}\right)$ coincides with the sample dosed with a polymer concentration of $7.0 \mathrm{mg} / \mathrm{L}$. This polymer concentration is in range of the optimum polymer dose determined through the preliminary turbidity results depicted in Figure 58, and the absorbance results depicted in Figure 62; both of which suggested the optimum dose measure $8.0 \mathrm{mg} / \mathrm{L}$. The preliminary settling test, however, suggested that the optimum dose measure close to $6.0 \mathrm{mg} / \mathrm{L}$. This is slightly different from the other results, however the settling test is based on visual appearance and settling time only, meaning it is very much an estimate and leaves room for error. The trends observed in all other results for this high turbidity water sample are once again observed in the particle count results, demonstrating an incremental decrease in total particle concentration with increasing polymer concentration towards the optimum concentration of $7.0 \mathrm{mg} / \mathrm{L}$. At the $4.0 \mathrm{mg} / \mathrm{L}$ polymer concentration, a slight decrease in total particle concentration is observed marginally outside of the trend. This may be due to 
inadequate mixing of the sample prior to measuring total particle concentration, as the samples following tend back to the expected trend. Beyond the optimum concentration, as the polymer dose is increased, the total particle concentration then increases incrementally to a value of $1.19 \times 10^{5} \#$ particles $/ \mathrm{mL}$ corresponding to the maximum polymer dose of $12.0 \mathrm{mg} / \mathrm{L}$, following the expected trend based on previous results.

Figures 65 shows very similar trends in the low turbidity water sample, illustrating that the lowest total particle concentration $\left(1.67 \times 10^{4} \#\right.$ particles $\left./ \mathrm{mL}\right)$ corresponds to a polymer concentration of $1.0 \mathrm{mg} / \mathrm{L}$. This polymer concentration differs slightly with the optimum polymer dose of $2.0 \mathrm{mg} / \mathrm{L}$ determined using the UV-vis spectroscopy absorbance method (Figure 63) and the preliminary settling test (Table 17) for the same sample. The preliminary turbidity results (Figure 59) suggested the optimum polymer dose be higher at a concentration of $8.0 \mathrm{mg} / \mathrm{L}$. Again, these preliminary results were likely not as sensitive as the absorbance and particle count results, as there was very marginal difference in turbidity between the $2.0 \mathrm{mg} / \mathrm{L}$ and $10.0 \mathrm{mg} / \mathrm{L}$ sample; whereas there is a more noticeable distinction between the difference in absorbance (0.453 A.U. and 0.599 A.U., respectively, using the in-line unit) and the difference in particle concentration $\left(1.67 \times 10^{4} \#\right.$ particles $/ \mathrm{mL}$ and $1.55 \times 10^{5}$ \#particles $/ \mathrm{mL}$, respectively) between the two concentrations. The variance in optimum dose results between the particle concentration $(1.0 \mathrm{mg} / \mathrm{L}$ polymer) and the UVvis absorbance $(2.0 \mathrm{mg} / \mathrm{L}$ polymer) suggests that the exact optimum dose lies in the range between these two concentrations. 
The particle count results for the high and low turbidity water samples dosed with Hydrex 3661 coincide with the absorbance results, as was observed for the samples dosed with Magnafloc LT27AG. This infers that UV-vis spectroscopy has the viability to be used as a tool for the optimization of polymer dose for not only an anionic Magnafloc LT27AG polymer but can also be replicated using a cationic Hydrex 3661 polymer. In addition, the ability of this method to succeed in a variety of different water matrices further demonstrates replicability.

Table 18 below outlines a complete comparison of optimum dosages for both polymers in each water sample matrix determined using each of the four analytical methods. For both polymers in all water matrices, the more robust UV-vis spectroscopy and particle concentration method of determining optimum dose produced result in accordance with one another. There is a very slight discrepancy between the two methods using the Hydrex 3661 polymer, the total particle concentration resulting in an optimum dosage $1.0 \mathrm{mg} / \mathrm{L}$ lower in both water samples. The settling test and turbidity measurements are also either in accordance or marginally variable to the UV-vis spectroscopy and particle concentration results for all samples and polymer types, with the exception of the turbidity results for the Hydrex 3661 polymer in the low turbidity water sample. The settling test and turbidity methods of determining optimum polymer dose are suitable to acquire a general polymer dose range, however the methods are quite rudimentary and are not expected to produce as precise of a result as UV-vis spectroscopy or total particle concentration 
Table 18: Comparison of optimum polymer dosages determined using the four methods in all water samples

\begin{tabular}{lccccc}
\hline \multicolumn{1}{c}{ Polymer } & \multicolumn{4}{c}{ Analytical Method } & \\
\hline & Sample Type & Settling Test & Turbidity & $\begin{array}{c}\text { UV-vis } \\
\text { absorbance }\end{array}$ & $\begin{array}{c}\text { Total particle } \\
\text { concentration }\end{array}$ \\
\hline Magnafloc LT27AG & High Turbidity & $2.00 \mathrm{mg} / \mathrm{L}$ & $2.00 \mathrm{mg} / \mathrm{L}$ & $2.00 \mathrm{mg} / \mathrm{L}$ & $2.00 \mathrm{mg} / \mathrm{L}$ \\
\hline Magnafloc LT27AG & Low Turbidity & $0.05 \mathrm{mg} / \mathrm{L}$ & $0.05 \mathrm{mg} / \mathrm{L}$ & $0.10 \mathrm{mg} / \mathrm{L}$ & $0.10 \mathrm{mg} / \mathrm{L}$ \\
\hline Magnafloc LT27AG & Actiflo & $0.50 \mathrm{mg} / \mathrm{L}$ & $1.00 \mathrm{mg} / \mathrm{L}$ & $0.50 \mathrm{mg} / \mathrm{L}$ & $0.50 \mathrm{mg} / \mathrm{L}$ \\
\hline Hydrex 3661 & High Turbidity & $8.00 \mathrm{mg} / \mathrm{L}$ & $6.00 \mathrm{mg} / \mathrm{L}$ & $8.00 \mathrm{mg} / \mathrm{L}$ & $7.00 \mathrm{mg} / \mathrm{L}$ \\
\hline Hydrex 3661 & Low Turbidity & $2.00 \mathrm{mg} / \mathrm{L}$ & $8.00 \mathrm{mg} / \mathrm{L}$ & $2.00 \mathrm{mg} / \mathrm{L}$ & $1.00 \mathrm{mg} / \mathrm{L}$ \\
\hline
\end{tabular}

\subsubsection{UV-vis absorbance as an in-line method for polymer optimization}

The results of this phase of the study indicate that an in-line UV-vis spectrophotometer not only has the potential to be used as a tool for the detection, quantification and monitoring of residual polymer in drinking water treatment applications; but also as a method for the in-line real-time determination of optimum polymer dose necessary for drinking water treatment applications. UV-vis spectroscopy has proven to be a suitable method for this purpose, seeing that the process can be continuously automated, and the need for additional chemical reagents and/or dilution is eliminated.

There is the potential to create a feedback control loop that would correlate the absorbance value of the water post-coagulation and flocculation to the concentration of residual polymer present in the water sample. The output could then be used for the continuous adjustment of the polymer dose being added in real-time, ensuring efficient coagulation, reduced polymer costs, and a decrease in the concentration of residual polymer being released into environmental water sources. In order for this full-scale system to be implemented, a minimum search algorithm would need to be developed. This algorithm 
would need to measure the change in slope between absorbance values coinciding with polymer concentrations as they vary, and minimize this slope through a polymer-dosing controller to maintain a constant optimum polymer dose. The development of this algorithm is outside the scope of this study, and will need to be explored through future work.

\subsection{Discussion of UV-vis spectroscopy in comparison to other analytical methods} for the detection of residual polymer in drinking water treatment applications

The results of experimental phases one, two and three demonstrate the viability of UV-vis spectroscopy as a sensitive method for the in-line detection of residual polymer in drinking water treatment applications, using the in-line spectrophotometer. These results also indicate that this method is suitable for water matrices containing particulate matter, as well as water matrices containing additional treatment chemicals used during drinking water treatment. As previously mentioned, the achievability of other analytical methods used for the detection of residual polymer in water treatment applications have also been explored.

High-performance liquid chromatography (HPLC) is an analytical method that has been researched extensively for the detection of residual acrylamide monomer in various water matrices. Brown and Read developed a HPLC/ultraviolet absorption detection procedure for the detection of acrylamide in samples of sea, river, potable and estuarine waters; as well as sewage and China clay works effluents. This method was able to achieve a detection range of $0.2-100 \mu \mathrm{g} / \mathrm{L}$ for acrylamide monomer, which at an assumed monomer content of 
$0.05 \%$ corresponds to a polyacrylamide concentration range of $4.0-2000 \mu \mathrm{g} / \mathrm{L}$ (51). This procedure involves the bromination and extraction of $\alpha, \beta$-dibromopropionamide with ethyl acetate, quantification using HPLC, and finally detection using ultraviolet detection, which is quite timely and impractical for use in-situ. Ver Vers developed a HPLC method using $\mathrm{C}_{18}$ and ion-exchange columns in series for the determination of acrylamide and acrylic monomers in polymeric samples and detection limits were shown in the parts-per-billion range; however again this method is more suitable for studying polymer degradation and less applicable for in-situ residual polymer detection (52). Backe et al. also explored the use of large-volume injection hydrophilic-interaction liquid chromatography and tandem mass spectrometry for the determination of acrylamide residuals in environmental and drinking waters. Interestingly, this study found that the concentration of acrylamide leaving the drinking water plant $(46 \mathrm{ng} / \mathrm{L})$ was about twice as high as that measured within the plant $(21-27 \mathrm{ng} / \mathrm{L})(53)$. It is mentioned that this event was not isolated, and that samples from a separate water treatment plant showed similar results. This was attributed to the fact that acrylamide does not have a large affinity for sludge and sediment, and therefore is not significantly reduced through clarification and/or filtration processes (54). This large amount of residual polyacrylamide was suspected to be degraded to its acrylamide monomer form through disinfection (chloroamination) or fluoridation processes (53). The advantage of using HPLC for the detection of residual polymer in drinking water treatment applications is the methods capability of detecting extremely low limits of isolated acrylamide monomer. The US EPA limit for the release of polyacrylamide is $1.0 \mathrm{mg} / \mathrm{L}$, corresponding to an acrylamide monomer concentration of $0.005 \mathrm{mg} / \mathrm{L}$ assuming a $0.05 \%$. monomer content. In terms of applicability, however, this method is quite cumbersome to 
perform in-situ and requires that the plant have the capacity to execute these procedures routinely.

Electron-capture gas chromatography is another analytical method that has been frequented for the detected of residual acrylamide monomers post-drinking water treatment. Croll and Simkins used a combination of $\alpha, \beta$-dibromopropionamide extraction using diethyl ether followed by analysis by electron-gas capture chromatography to detect residual acrylamide monomer in a river water sample. The detection limit of acrylamide monomer was found to be $0.1 \mu \mathrm{g} / \mathrm{L}$, which, at an assumed acrylamide monomer content of $0.05 \%$, results in a polyacrylamide detection limit of $2.0 \mu \mathrm{g} / \mathrm{L}$ (55). Yamini et al. performed a study developing and validating a method using dispersive liquid-liquid microextraction coupled with electron-capture gas chromatography detection to detect ultra-trace amounts of acrylamide contaminant in water samples filtered through a $0.45 \mu \mathrm{m}$ filter. This method was able to form a correlation between acrylamide concentration and the response of ECD with a correlation of determination $\left(\mathrm{R}^{2}\right)$ of 0.9999 , a relative standard deviation (RSD) of 3.6\%, and a limit of detection of $0.001 \mu \mathrm{g} / \mathrm{L}$ of acrylamide monomer, which corresponds to $0.02 \mu \mathrm{g} / \mathrm{L}$ of polyacrylamide polymer at a monomer content of $0.05 \%(56)$. As is similar for the high-performance liquid chromatography method, electron-capture gas chromatography does have the advantage of extremely low detection limits of acrylamide monomer. Again, this method does require plants to have the capacity to carry out these extensive analytical methods, and the option of continuous monitoring is not viable as is with UV-vis spectroscopy as a method for the detection of residual polymer post-drinking water treatment. 


\subsection{Discussion of determination of optimum polymer dose}

The use of UV-vis spectroscopy for the determination of optimum polymer dose is a method that has been previously examined for use in other industrial applications. Salam et al. explored the use of this method for the determination of optimum polymer dose in oil sands tailings. This study was very similar to that performed in this study on drinking water samples, however it was performed on oil sands tailings conditioned with an anionic A3338 (SNF Group, France) polymer dosed in both the under and overdose ranges. A capillary suction time (CST) test was performed on the conditioned samples as a comparison method to initially determine the optimum polymer dose for the tailings sample. Afterwards, a settling test was also performed on the conditioned samples. The supernatant of these settled samples was then used for UV-vis spectroscopy absorbance measurements, using a desktop spectrophotometer. The results of this study showed that the optimum polymer dose, determined by the lowest capillary suction time, aligned with the polymer dose that yielded the lowest absorbance value at 190nm (47). This experiment is quite similar to that performed on the drinking water samples in this study, however the trend in absorbance was instead correlated to the lowest particle concentration as well as the lowest turbidity measurements of the supernatant of the settled samples dosed with polymer. A CST test would not be appropriate for samples of drinking water, as these samples contain very little particulate matter and do not possess the necessary rheological characteristics to carry out a CST test. However, the correlation between minimum turbidity and particle concentration with minimum absorbance aligns very well with the results of the CST-absorbance results in the study done by Salam et al. 
As was previously mentioned, Al Momani and Örmeci also conducted a similar study on the use of UV-vis spectroscopy as a method for the optimization of sludge dewatering. This study conditioned anaerobically digested sludge samples with various doses of a few different cationic and anionic polymers. In this case, CST tests were coupled with filtration tests to quantify the filtration rate of the conditioned sludge samples. The filtrate of these samples was then used to carry out UV-vis spectroscopy absorbance measurements using an in-line UV-vis spectrophotometer. The results of this study concluded that samples dosed with polymer in the optimum dose range correlated with those that exhibited the lowest capillary suction time, fastest filtration rate and minimum absorbance at a wavelength at or around $191.5 \mathrm{~nm}$ (23). This study also showed that in the over and underdose ranges, the absorbance and CST results tended to the same $\mathrm{U}$ or V-shape as was demonstrated in the results found using drinking water samples. Again, this study further indicates that a sample, whether it be sludge or drinking water, dosed with the optimum concentration of polymer will tend to a minimum absorbance value at or around $190 \mathrm{~nm}$.

The correspondence between these studies further demonstrates not only the replicability of UV-vis spectroscopy as a method for monitoring polymer dose, but also the wide range of applications this method has the potential to expand upon. The release of residual polymer into the environment has negative impacts regardless of industrial source, and the negative impacts of polymer overdose and under-dose are experienced in a plethora of different applications. 


\section{CHAPTER 6: CONCLUSION}

The general conclusions determined from this study are as follows:

- The use of UV-vis spectroscopy as a method for the in-line detection of residual polyacrylamide polymer concentration was established in deionized water. The method was able to form a linear correlation between polymer concentration and absorbance at $190 \mathrm{~nm}$ at both a high $(0-3.0 \mathrm{mg} / \mathrm{L})$ and low $(0-1.0 \mathrm{mg} / \mathrm{L})$ polymer concentration range. Linear correlation coefficients of 0.9990 and 0.9753 were attained for the relationship between polymer concentration and absorbance at $190 \mathrm{~nm}$ for the high and low concentration ranges, respectively.

- The effect of path length on the sensitivity of in-line UV-vis spectroscopy as a method for the detection of residual polyacrylamide polymer in water was studied using dosed samples of deionized water. It was determined that a path length of $4 \mathrm{~mm}$ rendered the most sensitive results at a low concentration range $(0-1.0 \mathrm{mg} / \mathrm{L})$ of polyacrylamide polymer when compared to a $2 \mathrm{~mm}, 8 \mathrm{~mm}$, and $10 \mathrm{~mm}$ path length; yielding a linear relationship between polymer concentration and absorbance at $190 \mathrm{~nm}$ with a linear correlation coefficient of $\mathrm{R}^{2}=0.9753$. At a higher concentration range $(0-3.0 \mathrm{mg} / \mathrm{L})$, the $4 \mathrm{~mm}$ and $8 \mathrm{~mm}$ path lengths performed similarly, yielding linear relationships with correlation coefficients of $\mathrm{R}^{2}=0.9990$ and $\mathrm{R}^{2}=0.9992$, respectively. Seeing that drinking water treatment requires the accurate detection of polymers at very low concentrations $(<1.0 \mathrm{mg} / \mathrm{L})$, the $4 \mathrm{~mm}$ path length was chosen as most effective and was used for subsequent experimental phases. 
- The effect of suspended particulates present in raw water samples on the viability of UV-vis spectroscopy as a method for the in-line detection of residual polymer in drinking water applications was studied. It was found that raw water samples still resulted in strong linear correlations between polymer concentration and absorbance at $190 \mathrm{~nm}$ at both the high and low concentration ranges, with linear relation coefficients of $\mathrm{R}^{2}=0.9990$ and $\mathrm{R}^{2}=0.9757$, respectively. The samples were then filtered, diluted with deionized water, as well as a combination of both filtration and dilution. It was found that filtration and dilution decreased the strength of the linear correlation slightly, however a substantial linear relationship was still present at both the high and low concentration ranges using the in-line spectrophotometer. The in-line spectrophotometer was capable of producing linear correlations between polymer concentration and absorbance at $190 \mathrm{~nm}$ of 0.8435 post-filtration, 0.8190 post dilution, and 0.3614 post-filtration and dilution at the low polymer dose range $(0-1 \mathrm{mg} / \mathrm{L})$. At the high polymer dose range, the in-line unit was capable of attaining linear relation coefficients of 0.8352 post-filtration, 0.9856 post-dilution, and 0.9356 post-filtration and dilution.

- The effect of process chemicals on the sensitivity of UV-vis spectroscopy as a method for the in-line detection of residual polymer in drinking water treatment applications was also studied. The Actiflo ${ }^{\circledR}$ enhanced coagulation process was carried out lab scale using raw water samples, and a linear relationship between polymer concentration and absorbance at 190nm was still attainable using an inline spectrophotometer at both the high and low concentration ranges. However, the linear relation coefficients did decrease in the presence of Actiflo ${ }^{\circledR}$ process 
chemicals, reducing the linear relation coefficients to 0.4485 at the high concentration range and 0.5565 at the low concentration range using the in-line spectrophotometer, indicating that the sensitivity of the method is reduced.

- Finally, a relationship between the optimum polymer dosage and the absorbance of the dosed sample at $190 \mathrm{~nm}$ was established in raw water samples of both high and low turbidity using both an anionic and cationic polyelectrolyte polymer. The constructed relationship formed a ' $U$ ' shape trend, with the minimum absorbance value corresponding to the optimum polymer dose. The optimum polymer dose indicated by the UV-vis absorbance method at $190 \mathrm{~nm}$ was in close agreement with optimum polymer concentration detected by common settling tests, turbidity tests, and total particle concentration. The in-line spectrophotometer determined an optimum anionic polymer dose of $0.1 \mathrm{mg} / \mathrm{L}$ in the low turbidity water sample $\left(2.11 \times 10^{5} \#\right.$ particles $\left./ \mathrm{mL}\right), 2.0 \mathrm{mg} / \mathrm{L}$ in the high turbidity water sample $\left(2.36 \times 10^{5}\right.$ \#particles $/ \mathrm{mL}$ ), and $0.5 \mathrm{mg} / \mathrm{L}$ in the high turbidity water sample dose with enhanced coagulation process chemicals $\left(1.07 \times 10^{5} \quad \#\right.$ particles $\left./ \mathrm{mL}\right)$. The in-line spectrophotometer also determined an optimum cationic polymer dose of $8.0 \mathrm{mg} / \mathrm{L}$ for the high turbidity water sample $\left(2.19 \times 10^{4} \#\right.$ particles $\left./ \mathrm{mL}\right)$, and $2.0 \mathrm{mg} / \mathrm{L}$ for the low turbidity water sample $\left(2.13 \times 10^{4}\right.$ \#particles $\left./ \mathrm{mL}\right)$. In addition, seeing that this relationship was established using an in-line spectrophotometer, this relationship has the potential to be used to create a control loop for automatic adjustments of polymer dosed in drinking water in real-time. 


\section{APPENDICES}

\section{Appendix A: UV-vis absorbance data for experimental phase 1}

\begin{tabular}{|c|c|c|c|c|c|c|c|c|c|c|c|}
\hline \multicolumn{12}{|c|}{ Magnafloc in deionized water $(2018$} \\
\hline \multicolumn{12}{|c|}{ Desktop spectrophotometer (1 $\mathrm{cm}$ path length) } \\
\hline $\begin{array}{l}\text { Wavelength } \\
(\mathrm{nm})\end{array}$ & $0 \mathrm{mg} / \mathrm{L}$ & $\begin{array}{c}0.1 \\
\mathrm{mg} / \mathrm{L}\end{array}$ & $\begin{array}{c}0.2 \\
\mathrm{mg} / \mathrm{L}\end{array}$ & $\begin{array}{c}0.3 \\
\mathrm{mg} / \mathrm{L}\end{array}$ & $\begin{array}{c}0.4 \\
\mathrm{mg} / \mathrm{L}\end{array}$ & $\begin{array}{c}0.5 \\
\mathrm{mg} / \mathrm{L}\end{array}$ & $\begin{array}{c}0.6 \\
\mathrm{mg} / \mathrm{L}\end{array}$ & $\begin{array}{c}0.7 \\
\mathrm{mg} / \mathrm{L}\end{array}$ & $\begin{array}{c}0.8 \\
\mathrm{mg} / \mathrm{L}\end{array}$ & $\begin{array}{c}0.9 \\
\mathrm{mg} / \mathrm{L}\end{array}$ & $\begin{array}{c}1.0 \\
\mathrm{mg} / \mathrm{L}\end{array}$ \\
\hline 250 & 0.042 & 0.042 & 0.042 & 0.042 & 0.044 & 0.042 & 0.042 & 0.043 & 0.042 & 0.044 & 0.044 \\
\hline 249 & 0.045 & 0.046 & 0.045 & 0.046 & 0.047 & 0.046 & 0.046 & 0.047 & 0.046 & 0.047 & 0.047 \\
\hline 248 & 0.048 & 0.049 & 0.048 & 0.049 & 0.050 & 0.049 & 0.049 & 0.050 & 0.049 & 0.050 & 0.050 \\
\hline 247 & 0.052 & 0.052 & 0.052 & 0.052 & 0.054 & 0.052 & 0.052 & 0.053 & 0.052 & 0.054 & 0.054 \\
\hline 246 & 0.055 & 0.056 & 0.055 & 0.056 & 0.057 & 0.055 & 0.055 & 0.056 & 0.055 & 0.057 & 0.057 \\
\hline 245 & 0.058 & 0.059 & 0.058 & 0.058 & 0.060 & 0.058 & 0.058 & 0.059 & 0.058 & 0.060 & 0.060 \\
\hline 244 & 0.060 & 0.061 & 0.061 & 0.061 & 0.062 & 0.061 & 0.061 & 0.062 & 0.061 & 0.062 & 0.062 \\
\hline 243 & 0.062 & 0.063 & 0.063 & 0.063 & 0.065 & 0.063 & 0.063 & 0.064 & 0.063 & 0.064 & 0.064 \\
\hline 242 & 0.064 & 0.065 & 0.064 & 0.064 & 0.066 & 0.064 & 0.064 & 0.065 & 0.064 & 0.066 & 0.066 \\
\hline 241 & 0.065 & 0.065 & 0.065 & 0.065 & 0.067 & 0.065 & 0.065 & 0.066 & 0.065 & 0.067 & 0.067 \\
\hline 240 & 0.065 & 0.066 & 0.065 & 0.066 & 0.067 & 0.065 & 0.065 & 0.066 & 0.065 & 0.067 & 0.067 \\
\hline 239 & 0.065 & 0.066 & 0.065 & 0.065 & 0.067 & 0.065 & 0.065 & 0.066 & 0.065 & 0.067 & 0.067 \\
\hline 238 & 0.064 & 0.065 & 0.064 & 0.065 & 0.066 & 0.064 & 0.064 & 0.065 & 0.065 & 0.066 & 0.066 \\
\hline 237 & 0.063 & 0.064 & 0.063 & 0.064 & 0.065 & 0.063 & 0.063 & 0.064 & 0.063 & 0.065 & 0.065 \\
\hline 236 & 0.062 & 0.062 & 0.062 & 0.062 & 0.064 & 0.062 & 0.061 & 0.063 & 0.062 & 0.063 & 0.063 \\
\hline 235 & 0.060 & 0.061 & 0.060 & 0.060 & 0.062 & 0.060 & 0.060 & 0.061 & 0.060 & 0.062 & 0.062 \\
\hline 234 & 0.058 & 0.059 & 0.058 & 0.058 & 0.060 & 0.058 & 0.058 & 0.059 & 0.058 & 0.060 & 0.060 \\
\hline 233 & 0.056 & 0.057 & 0.056 & 0.056 & 0.058 & 0.056 & 0.056 & 0.057 & 0.056 & 0.058 & 0.058 \\
\hline 232 & 0.054 & 0.055 & 0.054 & 0.054 & 0.056 & 0.054 & 0.054 & 0.055 & 0.054 & 0.056 & 0.056 \\
\hline 231 & 0.052 & 0.053 & 0.052 & 0.052 & 0.054 & 0.052 & 0.052 & 0.053 & 0.052 & 0.054 & 0.054 \\
\hline 230 & 0.051 & 0.051 & 0.051 & 0.051 & 0.053 & 0.050 & 0.050 & 0.051 & 0.051 & 0.052 & 0.052 \\
\hline 229 & 0.049 & 0.050 & 0.049 & 0.049 & 0.051 & 0.049 & 0.049 & 0.050 & 0.049 & 0.051 & 0.051 \\
\hline 228 & 0.047 & 0.048 & 0.048 & 0.048 & 0.050 & 0.047 & 0.047 & 0.048 & 0.048 & 0.049 & 0.049 \\
\hline 227 & 0.046 & 0.047 & 0.046 & 0.046 & 0.049 & 0.046 & 0.046 & 0.047 & 0.046 & 0.048 & 0.048 \\
\hline 226 & 0.045 & 0.046 & 0.045 & 0.045 & 0.048 & 0.045 & 0.045 & 0.046 & 0.045 & 0.047 & 0.047 \\
\hline 225 & 0.045 & 0.046 & 0.045 & 0.045 & 0.047 & 0.045 & 0.045 & 0.045 & 0.045 & 0.047 & 0.047 \\
\hline 224 & 0.045 & 0.046 & 0.045 & 0.045 & 0.047 & 0.044 & 0.044 & 0.045 & 0.045 & 0.047 & 0.047 \\
\hline 223 & 0.045 & 0.046 & 0.045 & 0.045 & 0.047 & 0.045 & 0.045 & 0.046 & 0.045 & 0.047 & 0.047 \\
\hline 222 & 0.046 & 0.047 & 0.046 & 0.046 & 0.048 & 0.046 & 0.046 & 0.047 & 0.046 & 0.048 & 0.048 \\
\hline 221 & 0.047 & 0.048 & 0.047 & 0.047 & 0.050 & 0.047 & 0.047 & 0.048 & 0.047 & 0.049 & 0.049 \\
\hline 220 & 0.049 & 0.050 & 0.049 & 0.049 & 0.052 & 0.049 & 0.049 & 0.050 & 0.049 & 0.051 & 0.051 \\
\hline
\end{tabular}




\begin{tabular}{|c|c|c|c|c|c|c|c|c|c|c|c|}
\hline 219 & 0.051 & 0.053 & 0.052 & 0.052 & 0.054 & 0.052 & 0.052 & 0.053 & 0.052 & 0.054 & 0.054 \\
\hline 218 & 0.055 & 0.056 & 0.055 & 0.055 & 0.058 & 0.055 & 0.055 & 0.056 & 0.055 & 0.057 & 0.057 \\
\hline 217 & 0.058 & 0.060 & 0.058 & 0.059 & 0.061 & 0.058 & 0.059 & 0.059 & 0.058 & 0.061 & 0.061 \\
\hline 216 & 0.062 & 0.064 & 0.063 & 0.063 & 0.066 & 0.062 & 0.063 & 0.064 & 0.062 & 0.065 & 0.065 \\
\hline 215 & 0.067 & 0.068 & 0.067 & 0.067 & 0.070 & 0.067 & 0.067 & 0.068 & 0.067 & 0.069 & 0.070 \\
\hline 214 & 0.071 & 0.073 & 0.072 & 0.072 & 0.075 & 0.072 & 0.072 & 0.073 & 0.072 & 0.074 & 0.075 \\
\hline 213 & 0.077 & 0.078 & 0.077 & 0.077 & 0.080 & 0.077 & 0.077 & 0.078 & 0.077 & 0.080 & 0.080 \\
\hline 212 & 0.082 & 0.084 & 0.082 & 0.083 & 0.086 & 0.083 & 0.083 & 0.084 & 0.082 & 0.085 & 0.086 \\
\hline 211 & 0.088 & 0.090 & 0.088 & 0.089 & 0.092 & 0.089 & 0.089 & 0.090 & 0.088 & 0.091 & 0.092 \\
\hline 210 & 0.094 & 0.096 & 0.095 & 0.095 & 0.099 & 0.095 & 0.095 & 0.096 & 0.095 & 0.098 & 0.098 \\
\hline 209 & 0.100 & 0.102 & 0.101 & 0.102 & 0.105 & 0.101 & 0.101 & 0.102 & 0.101 & 0.104 & 0.105 \\
\hline 208 & 0.107 & 0.109 & 0.107 & 0.108 & 0.111 & 0.108 & 0.108 & 0.108 & 0.107 & 0.110 & 0.111 \\
\hline 207 & 0.113 & 0.115 & 0.113 & 0.114 & 0.118 & 0.114 & 0.114 & 0.115 & 0.113 & 0.116 & 0.118 \\
\hline 206 & 0.119 & 0.121 & 0.119 & 0.120 & 0.124 & 0.120 & 0.120 & 0.121 & 0.119 & 0.122 & 0.124 \\
\hline 205 & 0.125 & 0.126 & 0.125 & 0.126 & 0.130 & 0.125 & 0.125 & 0.126 & 0.125 & 0.128 & 0.130 \\
\hline 204 & 0.130 & 0.132 & 0.130 & 0.131 & 0.135 & 0.131 & 0.131 & 0.132 & 0.130 & 0.134 & 0.136 \\
\hline 203 & 0.135 & 0.137 & 0.135 & 0.136 & 0.141 & 0.136 & 0.136 & 0.137 & 0.135 & 0.139 & 0.141 \\
\hline 202 & 0.141 & 0.142 & 0.141 & 0.142 & 0.146 & 0.141 & 0.141 & 0.142 & 0.141 & 0.145 & 0.147 \\
\hline 201 & 0.146 & 0.147 & 0.146 & 0.147 & 0.151 & 0.147 & 0.146 & 0.148 & 0.146 & 0.150 & 0.153 \\
\hline 200 & 0.151 & 0.153 & 0.151 & 0.152 & 0.156 & 0.152 & 0.151 & 0.153 & 0.151 & 0.156 & 0.159 \\
\hline 199 & 0.156 & 0.158 & 0.156 & 0.157 & 0.162 & 0.157 & 0.157 & 0.158 & 0.157 & 0.162 & 0.165 \\
\hline 198 & 0.161 & 0.163 & 0.161 & 0.162 & 0.167 & 0.162 & 0.162 & 0.164 & 0.162 & 0.167 & 0.172 \\
\hline 197 & 0.167 & 0.168 & 0.167 & 0.167 & 0.173 & 0.168 & 0.168 & 0.170 & 0.168 & 0.174 & 0.179 \\
\hline 196 & 0.172 & 0.174 & 0.173 & 0.173 & 0.179 & 0.175 & 0.175 & 0.177 & 0.175 & 0.182 & 0.187 \\
\hline 195 & 0.180 & 0.182 & 0.180 & 0.181 & 0.187 & 0.183 & 0.183 & 0.186 & 0.184 & 0.191 & 0.197 \\
\hline 194 & 0.191 & 0.193 & 0.191 & 0.193 & 0.199 & 0.194 & 0.196 & 0.199 & 0.197 & 0.204 & 0.210 \\
\hline 193 & 0.201 & 0.203 & 0.202 & 0.204 & 0.210 & 0.206 & 0.208 & 0.210 & 0.209 & 0.216 & 0.223 \\
\hline 192 & 0.220 & 0.223 & 0.222 & 0.224 & 0.230 & 0.226 & 0.228 & 0.232 & 0.230 & 0.238 & 0.245 \\
\hline 191 & 0.251 & 0.254 & 0.252 & 0.255 & 0.262 & 0.258 & 0.261 & 0.264 & 0.261 & 0.271 & 0.278 \\
\hline 190 & 0.292 & 0.295 & 0.293 & 0.296 & 0.303 & 0.299 & 0.303 & 0.306 & 0.304 & 0.313 & 0.320 \\
\hline CONC & 0.000 & 0.100 & 0.200 & 0.300 & 0.400 & 0.500 & 0.600 & 0.700 & 0.800 & 0.900 & 1.000 \\
\hline $\begin{array}{l}\text { ABS AT } \\
190 \mathrm{~nm}\end{array}$ & 0.292 & 0.295 & 0.293 & 0.296 & 0.303 & 0.299 & 0.303 & 0.306 & 0.304 & 0.313 & 0.320 \\
\hline STDV & 0.002 & 0.000 & 0.001 & 0.001 & 0.001 & 0.001 & 0.002 & 0.000 & 0.001 & 0.000 & 0.001 \\
\hline
\end{tabular}




\begin{tabular}{|c|c|c|c|c|c|c|c|c|c|c|c|}
\hline \multicolumn{12}{|c|}{ Magnafloc in deionized water (2018-02-02) } \\
\hline \multicolumn{12}{|c|}{ In-line spectrophotometer ( $2 \mathrm{~mm}$ path length) } \\
\hline $\begin{array}{c}\text { Wavelength } \\
(\mathrm{nm})\end{array}$ & $0 \mathrm{mg} / \mathrm{L}$ & $\begin{array}{c}0.1 \\
\mathrm{mg} / \mathrm{L}\end{array}$ & $\begin{array}{c}0.2 \\
\mathrm{mg} / \mathrm{L}\end{array}$ & $\begin{array}{c}0.3 \\
\mathrm{mg} / \mathrm{L}\end{array}$ & $\begin{array}{c}0.4 \\
\mathrm{mg} / \mathrm{L}\end{array}$ & $\begin{array}{c}0.5 \\
\mathrm{mg} / \mathrm{L}\end{array}$ & $\begin{array}{c}0.6 \\
\mathrm{mg} / \mathrm{L} \\
\end{array}$ & $\begin{array}{c}0.7 \\
\mathrm{mg} / \mathrm{L}\end{array}$ & $\begin{array}{c}0.8 \\
\mathrm{mg} / \mathrm{L}\end{array}$ & $\begin{array}{c}0.9 \\
\mathrm{mg} / \mathrm{L}\end{array}$ & $\begin{array}{c}1.0 \\
\mathrm{mg} / \mathrm{L} \\
\end{array}$ \\
\hline 189.8 & -0.0002 & 0.0195 & 0.0195 & 0.0186 & 0.0157 & 0.0172 & 0.0197 & 0.0193 & 0.0203 & 0.0240 & 0.0229 \\
\hline 192.8 & -0.0001 & 0.0106 & 0.0103 & 0.0087 & 0.0064 & 0.0065 & 0.0068 & 0.0070 & 0.0067 & 0.0076 & 0.0084 \\
\hline 195.8 & 0.0009 & 0.0078 & 0.0057 & 0.0048 & 0.0031 & 0.0028 & 0.0007 & 0.0007 & 0.0025 & 0.0010 & 0.0014 \\
\hline 198.9 & -0.0001 & 0.0068 & 0.0039 & 0.0040 & 0.0009 & 0.0010 & -0.0011 & -0.0013 & 0.0005 & -0.0013 & -0.0012 \\
\hline 201.9 & -0.0008 & 0.0041 & 0.0019 & 0.0018 & -0.0018 & -0.0016 & -0.0024 & -0.0032 & -0.0022 & -0.0018 & -0.0037 \\
\hline 204.9 & -0.0023 & 0.0025 & 0.0017 & 0.0002 & -0.0021 & -0.0040 & -0.0044 & -0.0039 & -0.0050 & -0.0029 & -0.0048 \\
\hline 208 & -0.0008 & 0.0022 & 0.0022 & 0.0001 & -0.0014 & -0.0034 & -0.0022 & -0.0023 & -0.0048 & -0.0038 & -0.0045 \\
\hline 211 & 0.0007 & 0.0019 & 0.0015 & -0.0009 & -0.0013 & -0.0028 & -0.0029 & -0.0032 & -0.0037 & -0.0045 & -0.0048 \\
\hline 214 & 0.0007 & 0.0017 & -0.0003 & -0.0014 & -0.0023 & -0.0026 & -0.0047 & -0.0047 & -0.0042 & -0.0053 & -0.0053 \\
\hline 217 & -0.0008 & 0.0004 & -0.0017 & -0.0020 & -0.0032 & -0.0034 & -0.0047 & -0.0054 & -0.0046 & -0.0049 & -0.0060 \\
\hline 220 & -0.0021 & -0.0012 & -0.0021 & -0.0023 & -0.0043 & -0.0043 & -0.0048 & -0.0053 & -0.0057 & -0.0050 & -0.0067 \\
\hline 223.1 & -0.0007 & -0.0007 & -0.0005 & -0.0011 & -0.0033 & -0.0040 & -0.0031 & -0.0034 & -0.0048 & -0.0038 & -0.0054 \\
\hline 226.1 & 0.0006 & 0.0000 & 0.0001 & -0.0015 & -0.0027 & -0.0039 & -0.0030 & -0.0029 & -0.0039 & -0.0037 & -0.0043 \\
\hline 229.1 & 0.0006 & 0.0007 & 0.0000 & -0.0017 & -0.0023 & -0.0030 & -0.0032 & -0.0033 & -0.0033 & -0.0037 & -0.0039 \\
\hline 232.1 & -0.0001 & 0.0000 & -0.0012 & -0.0021 & -0.0029 & -0.0026 & -0.0036 & -0.0041 & -0.0034 & -0.0037 & -0.0047 \\
\hline 235.1 & -0.0008 & -0.0010 & -0.0016 & -0.0016 & -0.0025 & -0.0023 & -0.0034 & -0.0041 & -0.0028 & -0.0032 & -0.0049 \\
\hline 238.1 & -0.0007 & 0.0000 & -0.0009 & -0.0002 & -0.0029 & -0.0017 & -0.0021 & -0.0030 & -0.0026 & -0.0026 & -0.0043 \\
\hline 241.1 & 0.0015 & 0.0005 & 0.0006 & 0.0000 & -0.0013 & -0.0020 & -0.0019 & -0.0018 & -0.0020 & -0.0018 & -0.0034 \\
\hline 244.1 & 0.0016 & 0.0011 & 0.0007 & -0.0011 & -0.0018 & -0.0035 & -0.0022 & -0.0020 & -0.0029 & -0.0029 & -0.0034 \\
\hline 247.1 & -0.0001 & 0.0002 & -0.0011 & -0.0032 & -0.0036 & -0.0038 & -0.0042 & -0.0034 & -0.0036 & -0.0039 & -0.0043 \\
\hline 250.1 & -0.0027 & -0.0011 & -0.0024 & -0.0036 & -0.0040 & -0.0029 & -0.0038 & -0.0043 & -0.0038 & -0.0038 & -0.0054 \\
\hline $\mathrm{CONC}$ & 0.0 & 0.1 & 0.2 & 0.3 & 0.4 & 0.5 & 0.6 & 0.7 & 0.8 & 0.9 & 1.0 \\
\hline $\begin{array}{l}\text { ABS at } \\
190 \mathrm{~nm}\end{array}$ & -0.0002 & 0.0195 & 0.0195 & 0.0186 & 0.0157 & 0.0172 & 0.0197 & 0.0193 & 0.0203 & 0.0240 & 0.0229 \\
\hline STDV & 0.0000 & 0.0027 & 0.0018 & 0.0020 & 0.0017 & 0.0000 & 0.0014 & 0.0007 & 0.0001 & 0.0016 & 0.0001 \\
\hline
\end{tabular}




\begin{tabular}{|c|c|c|c|c|c|c|c|c|c|c|c|}
\hline \multicolumn{12}{|c|}{ Magnafloc in deionized water (2018-02-14) } \\
\hline \multicolumn{12}{|c|}{ In-line spectrophotometer ( $4 \mathrm{~mm}$ path length) } \\
\hline $\begin{array}{c}\text { Wavelength } \\
(\mathrm{nm})\end{array}$ & $0 \mathrm{mg} / \mathrm{L}$ & $\begin{array}{c}0.1 \\
\mathrm{mg} / \mathrm{L}\end{array}$ & $\begin{array}{c}0.2 \\
\mathrm{mg} / \mathrm{L}\end{array}$ & $\begin{array}{c}0.3 \\
\mathrm{mg} / \mathrm{L}\end{array}$ & $\begin{array}{c}0.4 \\
\mathrm{mg} / \mathrm{L}\end{array}$ & $\begin{array}{c}0.5 \\
\mathrm{mg} / \mathrm{L}\end{array}$ & $\begin{array}{c}0.6 \\
\mathrm{mg} / \mathrm{L}\end{array}$ & $\begin{array}{c}0.7 \\
\mathrm{mg} / \mathrm{L}\end{array}$ & $\begin{array}{c}0.8 \\
\mathrm{mg} / \mathrm{L}\end{array}$ & $\begin{array}{c}0.9 \\
\mathrm{mg} / \mathrm{L}\end{array}$ & $\begin{array}{c}1.0 \\
\mathrm{mg} / \mathrm{L}\end{array}$ \\
\hline 189.8 & 0.0000 & 0.0024 & 0.0050 & 0.0062 & 0.0083 & 0.0097 & 0.0122 & 0.0132 & 0.0151 & 0.0178 & 0.0231 \\
\hline 192.8 & -0.0006 & -0.0005 & 0.0003 & 0.0022 & 0.0030 & 0.0027 & 0.0053 & 0.0057 & 0.0063 & 0.0070 & 0.0107 \\
\hline 195.8 & 0.0004 & 0.0014 & 0.0020 & 0.0023 & 0.0025 & 0.0037 & 0.0035 & 0.0042 & 0.0041 & 0.0057 & 0.0079 \\
\hline 198.9 & -0.0001 & 0.0009 & 0.0013 & 0.0010 & 0.0018 & 0.0021 & 0.0025 & 0.0028 & 0.0033 & 0.0040 & 0.0054 \\
\hline 201.9 & 0.0000 & -0.0008 & -0.0007 & -0.0003 & -0.0001 & -0.0003 & 0.0007 & 0.0005 & 0.0005 & 0.0005 & 0.0021 \\
\hline 204.9 & 0.0007 & 0.0016 & 0.0017 & 0.0017 & 0.0011 & 0.0017 & 0.0014 & 0.0021 & 0.0018 & 0.0026 & 0.0025 \\
\hline 208 & -0.0003 & 0.0002 & 0.0004 & 0.0006 & 0.0009 & 0.0010 & 0.0013 & 0.0013 & 0.0014 & 0.0015 & 0.0022 \\
\hline 211 & -0.0003 & -0.0003 & -0.0007 & -0.0002 & -0.0001 & -0.0006 & 0.0001 & 0.0001 & -0.0002 & -0.0002 & 0.0007 \\
\hline 214 & 0.0003 & 0.0011 & 0.0013 & 0.0010 & 0.0007 & 0.0014 & 0.0008 & 0.0012 & 0.0011 & 0.0017 & 0.0015 \\
\hline 217 & -0.0005 & -0.0009 & -0.0008 & -0.0009 & -0.0006 & -0.0003 & -0.0004 & -0.0005 & -0.0005 & -0.0011 & -0.0002 \\
\hline 220 & 0.0004 & -0.0001 & -0.0003 & 0.0000 & 0.0000 & -0.0001 & 0.0000 & -0.0001 & -0.0001 & -0.0003 & 0.0002 \\
\hline 223.1 & 0.0001 & 0.0006 & 0.0009 & 0.0006 & 0.0005 & 0.0006 & 0.0006 & 0.0005 & 0.0003 & 0.0009 & 0.0008 \\
\hline 226.1 & -0.0003 & -0.0007 & -0.0011 & -0.0009 & -0.0007 & -0.0005 & -0.0007 & -0.0011 & -0.0008 & -0.0013 & -0.0006 \\
\hline 229.1 & 0.0005 & 0.0006 & 0.0007 & 0.0007 & 0.0006 & 0.0003 & 0.0004 & 0.0005 & 0.0002 & 0.0002 & 0.0008 \\
\hline 232.1 & -0.0001 & 0.0000 & 0.0005 & 0.0000 & -0.0001 & 0.0002 & 0.0001 & -0.0002 & -0.0002 & 0.0002 & -0.0004 \\
\hline 235.1 & -0.0002 & -0.0006 & -0.0007 & -0.0007 & -0.0001 & -0.0003 & -0.0005 & -0.0007 & -0.0008 & -0.0013 & -0.0006 \\
\hline 238.1 & 0.0009 & 0.0010 & 0.0010 & 0.0012 & 0.0009 & 0.0029 & 0.0010 & 0.0008 & 0.0007 & 0.0007 & 0.0007 \\
\hline 241.1 & -0.0002 & -0.0002 & -0.0003 & -0.0005 & -0.0006 & -0.0001 & -0.0005 & -0.0006 & -0.0010 & -0.0004 & -0.0009 \\
\hline 244.1 & -0.0004 & -0.0004 & -0.0008 & -0.0006 & -0.0003 & -0.0005 & -0.0008 & -0.0010 & -0.0008 & -0.0013 & -0.0007 \\
\hline 247.1 & 0.0007 & 0.0008 & 0.0009 & 0.0009 & 0.0009 & 0.0003 & 0.0005 & 0.0008 & 0.0004 & 0.0003 & 0.0007 \\
\hline 250.1 & -0.0001 & -0.0008 & -0.0005 & -0.0010 & -0.0012 & -0.0006 & -0.0008 & -0.0012 & -0.0014 & -0.0012 & -0.0015 \\
\hline $\mathrm{CONC}$ & 0 & 0.1 & 0.2 & 0.3 & 0.4 & 0.5 & 0.6 & 0.7 & 0.8 & 0.9 & 1 \\
\hline $\begin{array}{l}\text { ABS AT } \\
190 \mathrm{~nm}\end{array}$ & 0.000 & 0.002 & 0.005 & 0.006 & 0.008 & 0.010 & 0.012 & 0.013 & 0.015 & 0.018 & 0.023 \\
\hline STDV & 0.001 & 0.001 & 0.000 & 0.000 & 0.000 & 0.001 & 0.001 & 0.000 & 0.001 & 0.001 & 0.000 \\
\hline
\end{tabular}




\begin{tabular}{|c|c|c|c|c|c|c|c|c|c|c|c|}
\hline \multicolumn{12}{|c|}{ Magnafloc in deionized water (2018-02-07) } \\
\hline \multicolumn{12}{|c|}{ In-line spectrophotometer (8mm path length) } \\
\hline $\begin{array}{c}\text { Wavelength } \\
(\mathrm{nm})\end{array}$ & $0 \mathrm{mg} / \mathrm{L}$ & $\begin{array}{c}0.1 \\
\mathrm{mg} / \mathrm{L}\end{array}$ & $\begin{array}{c}0.2 \\
\mathrm{mg} / \mathrm{L}\end{array}$ & $\begin{array}{c}0.3 \\
\mathrm{mg} / \mathrm{L}\end{array}$ & $\begin{array}{c}0.4 \\
\mathrm{mg} / \mathrm{L}\end{array}$ & $\begin{array}{c}0.5 \\
\mathrm{mg} / \mathrm{L}\end{array}$ & $\begin{array}{c}0.6 \\
\mathrm{mg} / \mathrm{L}\end{array}$ & $\begin{array}{c}0.7 \\
\mathrm{mg} / \mathrm{L}\end{array}$ & $\begin{array}{c}0.8 \\
\mathrm{mg} / \mathrm{L}\end{array}$ & $\begin{array}{c}0.9 \\
\mathrm{mg} / \mathrm{L}\end{array}$ & $\begin{array}{c}1.0 \\
\mathrm{mg} / \mathrm{L}\end{array}$ \\
\hline 189.8 & 0.0000 & 0.0000 & -0.0004 & 0.0027 & 0.0041 & 0.0056 & 0.0075 & 0.0089 & 0.0095 & 0.0122 & 0.0145 \\
\hline 192.8 & 0.0000 & -0.0003 & -0.0006 & 0.0005 & 0.0011 & 0.0019 & 0.0030 & 0.0037 & 0.0040 & 0.0056 & 0.0067 \\
\hline 195.8 & 0.0001 & 0.0001 & -0.0003 & 0.0001 & 0.0003 & 0.0008 & 0.0012 & 0.0015 & 0.0013 & 0.0025 & 0.0029 \\
\hline 198.9 & -0.0003 & -0.0003 & -0.0011 & -0.0001 & -0.0003 & -0.0002 & -0.0001 & 0.0002 & -0.0003 & 0.0005 & 0.0006 \\
\hline 201.9 & -0.0003 & -0.0005 & -0.0012 & -0.0004 & -0.0005 & -0.0003 & -0.0006 & -0.0004 & -0.0010 & -0.0007 & -0.0001 \\
\hline 204.9 & -0.0004 & -0.0008 & -0.0015 & -0.0003 & -0.0005 & -0.0008 & -0.0008 & -0.0008 & -0.0012 & -0.0010 & -0.0006 \\
\hline 208 & -0.0004 & -0.0005 & -0.0009 & -0.0004 & -0.0003 & -0.0009 & -0.0008 & -0.0009 & -0.0010 & -0.0007 & -0.0006 \\
\hline 211 & -0.0002 & 0.0001 & -0.0001 & -0.0003 & -0.0004 & -0.0006 & -0.0006 & -0.0006 & -0.0007 & -0.0005 & -0.0005 \\
\hline 214 & 0.0001 & 0.0006 & 0.0006 & 0.0001 & 0.0000 & -0.0001 & -0.0002 & -0.0003 & -0.0004 & -0.0003 & -0.0001 \\
\hline 217 & 0.0000 & 0.0003 & 0.0005 & -0.0001 & -0.0002 & -0.0001 & -0.0003 & -0.0005 & -0.0006 & -0.0006 & -0.0005 \\
\hline 220 & -0.0001 & -0.0001 & 0.0000 & -0.0001 & -0.0004 & -0.0003 & -0.0006 & -0.0006 & -0.0010 & -0.0010 & -0.0009 \\
\hline 223.1 & -0.0001 & -0.0003 & -0.0006 & 0.0000 & -0.0005 & -0.0004 & -0.0006 & -0.0007 & -0.0011 & -0.0010 & -0.0010 \\
\hline 226.1 & -0.0001 & 0.0000 & -0.0005 & 0.0003 & -0.0001 & -0.0004 & -0.0005 & -0.0006 & -0.0008 & -0.0005 & -0.0008 \\
\hline 229.1 & -0.0005 & -0.0001 & -0.0005 & -0.0002 & -0.0004 & -0.0006 & -0.0006 & -0.0008 & -0.0012 & -0.0008 & -0.0008 \\
\hline 232.1 & -0.0003 & 0.0002 & 0.0000 & -0.0002 & -0.0002 & -0.0004 & -0.0005 & -0.0006 & -0.0009 & -0.0008 & -0.0005 \\
\hline 235.1 & 0.0002 & 0.0006 & 0.0007 & 0.0000 & 0.0001 & 0.0000 & -0.0001 & -0.0006 & -0.0004 & -0.0006 & -0.0002 \\
\hline 238.1 & 0.0004 & 0.0004 & 0.0007 & 0.0002 & 0.0000 & 0.0001 & -0.0001 & -0.0004 & -0.0004 & -0.0006 & -0.0004 \\
\hline 241.1 & 0.0003 & 0.0004 & 0.0006 & 0.0003 & 0.0001 & 0.0000 & -0.0001 & -0.0004 & -0.0005 & -0.0004 & -0.0006 \\
\hline 244.1 & -0.0001 & 0.0001 & 0.0002 & 0.0005 & 0.0001 & -0.0003 & -0.0003 & -0.0004 & -0.0007 & -0.0003 & -0.0008 \\
\hline 247.1 & -0.0003 & 0.0004 & -0.0002 & 0.0005 & 0.0001 & 0.0000 & -0.0001 & -0.0004 & -0.0006 & -0.0003 & -0.0005 \\
\hline 250.1 & -0.0006 & 0.0003 & -0.0001 & 0.0002 & 0.0001 & -0.0001 & -0.0002 & -0.0003 & -0.0005 & -0.0005 & -0.0003 \\
\hline $\mathrm{CONC}$ & 0.0 & 0.1 & 0.2 & 0.3 & 0.4 & 0.5 & 0.6 & 0.7 & 0.8 & 0.9 & 1.0 \\
\hline $\begin{array}{l}\text { ABS at } \\
190 \mathrm{NM}\end{array}$ & 0.0000 & 0.0000 & -0.0004 & 0.0027 & 0.0041 & 0.0056 & 0.0075 & 0.0089 & 0.0095 & 0.0122 & 0.0145 \\
\hline STDV & 0.0006 & 0.0002 & 0.0002 & 0.0001 & 0.0005 & 0.0003 & 0.0002 & 0.0001 & 0.0003 & 0.0008 & 0.0000 \\
\hline
\end{tabular}




\begin{tabular}{|c|c|c|c|c|c|c|c|}
\hline \multicolumn{8}{|c|}{ Magnafloc in deionized water (2018-01-08) } \\
\hline \multicolumn{8}{|c|}{ Desktop spectrophotometer (1 $\mathrm{cm}$ path length) } \\
\hline Wavelength (nm) & $0 \mathrm{mg} / \mathrm{L}$ & $0.5 \mathrm{mg} / \mathrm{L}$ & $1.0 \mathrm{mg} / \mathrm{L}$ & $1.5 \mathrm{mg} / \mathrm{L}$ & $2.0 \mathrm{mg} / \mathrm{L}$ & $2.5 \mathrm{mg} / \mathrm{L}$ & $3.0 \mathrm{mg} / \mathrm{L}$ \\
\hline 250 & 0.0420 & 0.0427 & 0.0420 & 0.0421 & 0.0430 & 0.0438 & 0.0423 \\
\hline 249 & 0.0454 & 0.0463 & 0.0455 & 0.0456 & 0.0464 & 0.0475 & 0.0458 \\
\hline 248 & 0.0487 & 0.0493 & 0.0486 & 0.0486 & 0.0497 & 0.0504 & 0.0489 \\
\hline 247 & 0.0520 & 0.0529 & 0.0520 & 0.0520 & 0.0529 & 0.0538 & 0.0523 \\
\hline 246 & 0.0553 & 0.0560 & 0.0551 & 0.0552 & 0.0562 & 0.0569 & 0.0554 \\
\hline 245 & 0.0580 & 0.0589 & 0.0579 & 0.0581 & 0.0591 & 0.0597 & 0.0583 \\
\hline 244 & 0.0605 & 0.0612 & 0.0603 & 0.0604 & 0.0616 & 0.0621 & 0.0609 \\
\hline 243 & 0.0626 & 0.0633 & 0.0625 & 0.0627 & 0.0637 & 0.0641 & 0.0630 \\
\hline 242 & 0.0641 & 0.0646 & 0.0639 & 0.0641 & 0.0651 & 0.0657 & 0.0644 \\
\hline 241 & 0.0647 & 0.0656 & 0.0647 & 0.0649 & 0.0660 & 0.0666 & 0.0651 \\
\hline 240 & 0.0651 & 0.0657 & 0.0651 & 0.0652 & 0.0662 & 0.0668 & 0.0655 \\
\hline 239 & 0.0648 & 0.0655 & 0.0648 & 0.0649 & 0.0661 & 0.0667 & 0.0654 \\
\hline 238 & 0.0641 & 0.0648 & 0.0640 & 0.0644 & 0.0652 & 0.0661 & 0.0646 \\
\hline 237 & 0.0630 & 0.0637 & 0.0629 & 0.0631 & 0.0645 & 0.0649 & 0.0636 \\
\hline 236 & 0.0614 & 0.0622 & 0.0615 & 0.0618 & 0.0629 & 0.0635 & 0.0623 \\
\hline 235 & 0.0597 & 0.0606 & 0.0599 & 0.0601 & 0.0613 & 0.0618 & 0.0606 \\
\hline 234 & 0.0579 & 0.0587 & 0.0580 & 0.0583 & 0.0592 & 0.0601 & 0.0588 \\
\hline 233 & 0.0558 & 0.0568 & 0.0561 & 0.0564 & 0.0576 & 0.0582 & 0.0571 \\
\hline 232 & 0.0539 & 0.0548 & 0.0541 & 0.0545 & 0.0558 & 0.0563 & 0.0551 \\
\hline 231 & 0.0519 & 0.0529 & 0.0522 & 0.0526 & 0.0538 & 0.0545 & 0.0533 \\
\hline 230 & 0.0502 & 0.0512 & 0.0505 & 0.0508 & 0.0521 & 0.0531 & 0.0516 \\
\hline 229 & 0.0484 & 0.0496 & 0.0487 & 0.0492 & 0.0505 & 0.0514 & 0.0501 \\
\hline 228 & 0.0470 & 0.0481 & 0.0474 & 0.0480 & 0.0492 & 0.0503 & 0.0489 \\
\hline 227 & 0.0456 & 0.0469 & 0.0463 & 0.0469 & 0.0481 & 0.0492 & 0.0480 \\
\hline 226 & 0.0447 & 0.0461 & 0.0454 & 0.0461 & 0.0474 & 0.0487 & 0.0474 \\
\hline 225 & 0.0441 & 0.0456 & 0.0450 & 0.0458 & 0.0470 & 0.0484 & 0.0470 \\
\hline 224 & 0.0440 & 0.0456 & 0.0450 & 0.0458 & 0.0471 & 0.0486 & 0.0474 \\
\hline 223 & 0.0443 & 0.0461 & 0.0455 & 0.0463 & 0.0477 & 0.0493 & 0.0481 \\
\hline 222 & 0.0451 & 0.0470 & 0.0465 & 0.0472 & 0.0488 & 0.0505 & 0.0493 \\
\hline 221 & 0.0465 & 0.0485 & 0.0480 & 0.0488 & 0.0504 & 0.0523 & 0.0510 \\
\hline 220 & 0.0484 & 0.0504 & 0.0501 & 0.0510 & 0.0528 & 0.0546 & 0.0535 \\
\hline 219 & 0.0509 & 0.0532 & 0.0528 & 0.0537 & 0.0555 & 0.0577 & 0.0565 \\
\hline 218 & 0.0538 & 0.0564 & 0.0561 & 0.0572 & 0.0590 & 0.0614 & 0.0602 \\
\hline 217 & 0.0575 & 0.0601 & 0.0598 & 0.0610 & 0.0629 & 0.0655 & 0.0642 \\
\hline 216 & 0.0616 & 0.0644 & 0.0641 & 0.0653 & 0.0676 & 0.0701 & 0.0690 \\
\hline 215 & 0.0658 & 0.0689 & 0.0687 & 0.0701 & 0.0726 & 0.0752 & 0.0741 \\
\hline
\end{tabular}




\begin{tabular}{|c|c|c|c|c|c|c|c|}
\hline 214 & 0.0709 & 0.0741 & 0.0738 & 0.0753 & 0.0780 & 0.0807 & 0.0798 \\
\hline 213 & 0.0761 & 0.0792 & 0.0791 & 0.0806 & 0.0834 & 0.0864 & 0.0854 \\
\hline 212 & 0.0814 & 0.0850 & 0.0849 & 0.0866 & 0.0897 & 0.0927 & 0.0919 \\
\hline 211 & 0.0873 & 0.0909 & 0.0909 & 0.0929 & 0.0960 & 0.0994 & 0.0986 \\
\hline 210 & 0.0936 & 0.0973 & 0.0975 & 0.0995 & 0.1028 & 0.1063 & 0.1056 \\
\hline 209 & 0.0998 & 0.1035 & 0.1038 & 0.1062 & 0.1096 & 0.1133 & 0.1126 \\
\hline 208 & 0.1061 & 0.1100 & 0.1102 & 0.1127 & 0.1166 & 0.1204 & 0.1198 \\
\hline 207 & 0.1120 & 0.1162 & 0.1164 & 0.1193 & 0.1231 & 0.1274 & 0.1268 \\
\hline 206 & 0.1181 & 0.1223 & 0.1226 & 0.1257 & 0.1299 & 0.1341 & 0.1339 \\
\hline 205 & 0.1236 & 0.1279 & 0.1286 & 0.1319 & 0.1365 & 0.1409 & 0.1410 \\
\hline 204 & 0.1288 & 0.1336 & 0.1342 & 0.1381 & 0.1430 & 0.1479 & 0.1482 \\
\hline 203 & 0.1338 & 0.1388 & 0.1397 & 0.1442 & 0.1494 & 0.1547 & 0.1557 \\
\hline 202 & 0.1387 & 0.1440 & 0.1454 & 0.1506 & 0.1561 & 0.1619 & 0.1636 \\
\hline 201 & 0.1437 & 0.1495 & 0.1515 & 0.1574 & 0.1633 & 0.1699 & 0.1723 \\
\hline 200 & 0.1482 & 0.1547 & 0.1574 & 0.1642 & 0.1706 & 0.1782 & 0.1818 \\
\hline 199 & 0.1529 & 0.1601 & 0.1636 & 0.1718 & 0.1785 & 0.1874 & 0.1920 \\
\hline 198 & 0.1574 & 0.1656 & 0.1701 & 0.1796 & 0.1872 & 0.1970 & 0.2032 \\
\hline 197 & 0.1625 & 0.1721 & 0.1776 & 0.1887 & 0.1969 & 0.2084 & 0.2161 \\
\hline 196 & 0.1682 & 0.1790 & 0.1856 & 0.1986 & 0.2077 & 0.2210 & 0.2305 \\
\hline 195 & 0.1756 & 0.1876 & 0.1958 & 0.2107 & 0.2207 & 0.2356 & 0.2467 \\
\hline 194 & 0.1865 & 0.2007 & 0.2102 & 0.2273 & 0.2382 & 0.2554 & 0.2684 \\
\hline 193 & 0.1972 & 0.2133 & 0.2242 & 0.2434 & 0.2556 & 0.2746 & 0.2892 \\
\hline 192 & 0.2171 & 0.2357 & 0.2482 & 0.2696 & 0.2827 & 0.3041 & 0.3206 \\
\hline 191 & 0.2486 & 0.2708 & 0.2848 & 0.3081 & 0.3226 & 0.3465 & 0.3646 \\
\hline 190 & 0.2902 & 0.3167 & 0.3322 & 0.3573 & 0.3733 & 0.3999 & 0.4194 \\
\hline CONC & 0.0 & 0.5 & 1.0 & 1.5 & 2.0 & 2.5 & 3.0 \\
\hline ABS AT 190NM & 0.2902 & 0.3167 & 0.3322 & 0.3573 & 0.3733 & 0.3999 & 0.4194 \\
\hline STDV & 0.0017 & 0.0004 & 0.0011 & 0.0008 & 0.0002 & 0.0013 & 0.0004 \\
\hline
\end{tabular}




\begin{tabular}{|c|c|c|c|c|c|c|c|}
\hline \multicolumn{8}{|c|}{ Magnafloc in deionized water (2018-02-13) } \\
\hline \multicolumn{8}{|c|}{ In-line spectrophotometer ( $2 \mathrm{~mm}$ path length) } \\
\hline Wavelength (nm) & $0 \mathrm{mg} / \mathrm{L}$ & $0.5 \mathrm{mg} / \mathrm{L}$ & $1.0 \mathrm{mg} / \mathrm{L}$ & $1.5 \mathrm{mg} / \mathrm{L}$ & $2.0 \mathrm{mg} / \mathrm{L}$ & $2.5 \mathrm{mg} / \mathrm{L}$ & $3.0 \mathrm{mg} / \mathrm{L}$ \\
\hline 189.8 & -0.0001 & 0.0017 & 0.0069 & 0.0101 & 0.0175 & 0.0216 & 0.0324 \\
\hline 192.8 & 0.0008 & 0.0010 & 0.0043 & 0.0047 & 0.0095 & 0.0116 & 0.0176 \\
\hline 195.8 & -0.0009 & 0.0007 & 0.0032 & 0.0035 & 0.0056 & 0.0083 & 0.0126 \\
\hline 198.9 & -0.0032 & -0.0027 & -0.0015 & -0.0007 & -0.0004 & 0.0022 & 0.0045 \\
\hline 201.9 & -0.0025 & -0.0042 & -0.0042 & -0.0018 & -0.0027 & -0.0014 & 0.0010 \\
\hline 204.9 & -0.0016 & -0.0036 & -0.0050 & -0.0036 & -0.0038 & -0.0038 & -0.0010 \\
\hline 208 & 0.0000 & -0.0023 & -0.0025 & -0.0017 & -0.0023 & -0.0034 & 0.0000 \\
\hline 211 & 0.0003 & 0.0006 & -0.0004 & -0.0012 & -0.0002 & -0.0015 & 0.0011 \\
\hline 214 & 0.0003 & 0.0019 & 0.0009 & -0.0013 & 0.0004 & -0.0004 & 0.0005 \\
\hline 217 & -0.0015 & -0.0002 & -0.0005 & -0.0025 & -0.0010 & -0.0009 & 0.0001 \\
\hline 220 & -0.0018 & -0.0019 & -0.0024 & -0.0025 & -0.0028 & -0.0025 & -0.0020 \\
\hline 223.1 & -0.0019 & -0.0039 & -0.0042 & -0.0032 & -0.0046 & -0.0045 & -0.0034 \\
\hline 226.1 & -0.0011 & -0.0039 & -0.0037 & -0.0029 & -0.0047 & -0.0045 & -0.0035 \\
\hline 229.1 & 0.0002 & -0.0009 & -0.0017 & -0.0012 & -0.0021 & -0.0030 & -0.0018 \\
\hline 232.1 & 0.0008 & 0.0007 & 0.0000 & -0.0006 & -0.0002 & -0.0013 & -0.0009 \\
\hline 235.1 & -0.0006 & 0.0020 & 0.0004 & -0.0006 & 0.0001 & -0.0010 & -0.0008 \\
\hline 238.1 & -0.0010 & 0.0006 & -0.0003 & -0.0019 & -0.0009 & -0.0010 & -0.0007 \\
\hline 241.1 & -0.0021 & -0.0016 & -0.0024 & -0.0030 & -0.0031 & -0.0031 & -0.0020 \\
\hline 244.1 & -0.0018 & -0.0036 & -0.0035 & -0.0036 & -0.0046 & -0.0040 & -0.0032 \\
\hline 247.1 & -0.0002 & -0.0037 & -0.0037 & -0.0030 & -0.0044 & -0.0039 & -0.0036 \\
\hline 250.1 & 0.0015 & -0.0010 & -0.0013 & -0.0001 & -0.0017 & -0.0025 & -0.0021 \\
\hline CONC & 0 & 0.5 & 1 & 1.5 & 2 & 2.5 & 3 \\
\hline ABS AT 190NM & -0.0001 & 0.0017 & 0.0069 & 0.0101 & 0.0175 & 0.0216 & 0.0324 \\
\hline STDV & 0.0005 & 0.0011 & 0.0006 & 0.0005 & 0.0015 & 0.0018 & 0.0025 \\
\hline
\end{tabular}




\begin{tabular}{|c|c|c|c|c|c|c|c|}
\hline \multicolumn{8}{|c|}{ Magnafloc in deionized water (2018-02-09) } \\
\hline \multicolumn{8}{|c|}{ In-line spectrophotometer (4mm path length) } \\
\hline Wavelength (nm) & $0 \mathrm{mg} / \mathrm{L}$ & $0.5 \mathrm{mg} / \mathrm{L}$ & $1.0 \mathrm{mg} / \mathrm{L}$ & $1.5 \mathrm{mg} / \mathrm{L}$ & $2.0 \mathrm{mg} / \mathrm{L}$ & $2.5 \mathrm{mg} / \mathrm{L}$ & $3.0 \mathrm{mg} / \mathrm{L}$ \\
\hline 189.8 & -0.0001 & 0.0105 & 0.0211 & 0.0296 & 0.0397 & 0.0506 & 0.0611 \\
\hline 192.8 & 0.0018 & 0.0065 & 0.0118 & 0.0179 & 0.0232 & 0.0296 & 0.0359 \\
\hline 195.8 & 0.0019 & 0.0043 & 0.0064 & 0.0113 & 0.0141 & 0.0185 & 0.0214 \\
\hline 198.9 & 0.0005 & 0.0021 & 0.0038 & 0.0070 & 0.0081 & 0.0108 & 0.0129 \\
\hline 201.9 & -0.0016 & 0.0004 & 0.0021 & 0.0037 & 0.0049 & 0.0069 & 0.0081 \\
\hline 204.9 & -0.0011 & 0.0007 & 0.0022 & 0.0027 & 0.0044 & 0.0055 & 0.0072 \\
\hline 208 & 0.0011 & 0.0014 & 0.0027 & 0.0034 & 0.0043 & 0.0051 & 0.0067 \\
\hline 211 & 0.0019 & 0.0019 & 0.0018 & 0.0034 & 0.0033 & 0.0042 & 0.0047 \\
\hline 214 & 0.0001 & 0.0004 & 0.0005 & 0.0018 & 0.0015 & 0.0025 & 0.0028 \\
\hline 217 & -0.0016 & -0.0010 & -0.0004 & 0.0003 & -0.0001 & 0.0012 & 0.0008 \\
\hline 220 & -0.0011 & -0.0006 & 0.0007 & 0.0003 & 0.0002 & 0.0008 & 0.0013 \\
\hline 223.1 & 0.0009 & 0.0005 & 0.0013 & 0.0011 & 0.0013 & 0.0013 & 0.0020 \\
\hline 226.1 & 0.0012 & 0.0007 & 0.0005 & 0.0012 & 0.0008 & 0.0004 & 0.0012 \\
\hline 229.1 & 0.0000 & -0.0004 & -0.0006 & 0.0000 & -0.0004 & -0.0004 & 0.0000 \\
\hline 232.1 & -0.0017 & -0.0010 & -0.0007 & -0.0009 & -0.0011 & -0.0006 & -0.0012 \\
\hline 235.1 & -0.0007 & -0.0005 & 0.0003 & -0.0003 & -0.0005 & 0.0000 & 0.0001 \\
\hline 238.1 & 0.0010 & 0.0003 & 0.0011 & 0.0007 & 0.0004 & 0.0004 & 0.0005 \\
\hline 241.1 & 0.0017 & 0.0010 & 0.0008 & 0.0014 & 0.0011 & 0.0007 & 0.0010 \\
\hline 244.1 & -0.0002 & -0.0009 & -0.0011 & -0.0005 & -0.0005 & -0.0012 & -0.0010 \\
\hline 247.1 & -0.0019 & -0.0017 & -0.0011 & -0.0013 & -0.0014 & -0.0017 & -0.0016 \\
\hline 250.1 & -0.0004 & -0.0008 & 0.0002 & -0.0002 & -0.0011 & -0.0002 & -0.0011 \\
\hline $\mathrm{CONC}$ & 0 & 0.5 & 1 & 1.5 & 2 & 2.5 & 3 \\
\hline ABS at $190 \mathrm{~nm}$ & -0.00010 & 0.01055 & 0.02109 & 0.02959 & 0.03966 & 0.05057 & 0.06111 \\
\hline STDV & 0.00069 & 0.00079 & 0.00109 & 0.00066 & 0.00063 & 0.00123 & 0.00031 \\
\hline
\end{tabular}




\begin{tabular}{|c|c|c|c|c|c|c|c|}
\hline \multicolumn{8}{|c|}{ Magnafloc in deionized water (2018-01-24) } \\
\hline \multicolumn{8}{|c|}{ In-line spectrophotometer (8mm path length) } \\
\hline Wavelength (nm) & $0 \mathrm{mg} / \mathrm{L}$ & $0.5 \mathrm{mg} / \mathrm{L}$ & $1.0 \mathrm{mg} / \mathrm{L}$ & $1.5 \mathrm{mg} / \mathrm{L}$ & $2.0 \mathrm{mg} / \mathrm{L}$ & $2.5 \mathrm{mg} / \mathrm{L}$ & $3.0 \mathrm{mg} / \mathrm{L}$ \\
\hline 189.8 & 0.0002 & 0.0445 & 0.0927 & 0.1456 & 0.1922 & 0.2327 & 0.2891 \\
\hline 192.8 & -0.0003 & 0.0281 & 0.0536 & 0.0849 & 0.1119 & 0.1372 & 0.1682 \\
\hline 195.8 & 0.0002 & 0.0192 & 0.0325 & 0.0520 & 0.0687 & 0.0844 & 0.1009 \\
\hline 198.9 & -0.0003 & 0.0135 & 0.0216 & 0.0333 & 0.0435 & 0.0543 & 0.0633 \\
\hline 201.9 & -0.0012 & 0.0076 & 0.0134 & 0.0225 & 0.0273 & 0.0355 & 0.0426 \\
\hline 204.9 & -0.0019 & 0.0039 & 0.0079 & 0.0150 & 0.0189 & 0.0250 & 0.0312 \\
\hline 208 & -0.0015 & 0.0030 & 0.0065 & 0.0119 & 0.0141 & 0.0191 & 0.0245 \\
\hline 211 & -0.0010 & 0.0043 & 0.0066 & 0.0107 & 0.0123 & 0.0162 & 0.0205 \\
\hline 214 & -0.0006 & 0.0043 & 0.0056 & 0.0084 & 0.0104 & 0.0129 & 0.0150 \\
\hline 217 & 0.0001 & 0.0058 & 0.0056 & 0.0074 & 0.0084 & 0.0109 & 0.0110 \\
\hline 220 & -0.0006 & 0.0036 & 0.0032 & 0.0049 & 0.0048 & 0.0065 & 0.0064 \\
\hline 223.1 & -0.0010 & 0.0024 & 0.0011 & 0.0016 & 0.0022 & 0.0034 & 0.0035 \\
\hline 226.1 & -0.0010 & 0.0014 & -0.0003 & 0.0011 & 0.0001 & 0.0006 & 0.0020 \\
\hline 229.1 & -0.0007 & 0.0008 & -0.0003 & 0.0002 & -0.0006 & -0.0004 & 0.0014 \\
\hline 232.1 & -0.0003 & 0.0015 & 0.0008 & 0.0001 & 0.0008 & 0.0004 & 0.0014 \\
\hline 235.1 & 0.0003 & 0.0019 & 0.0014 & -0.0001 & 0.0019 & 0.0015 & 0.0007 \\
\hline 238.1 & 0.0011 & 0.0041 & 0.0026 & 0.0017 & 0.0025 & 0.0025 & 0.0021 \\
\hline 241.1 & 0.0001 & 0.0030 & 0.0012 & 0.0013 & 0.0008 & 0.0018 & 0.0006 \\
\hline 244.1 & -0.0007 & 0.0018 & 0.0003 & 0.0006 & -0.0009 & -0.0001 & -0.0006 \\
\hline 247.1 & -0.0012 & -0.0005 & -0.0013 & -0.0012 & -0.0028 & -0.0036 & -0.0022 \\
\hline 250.1 & -0.0001 & 0.0001 & -0.0008 & -0.0025 & -0.0020 & -0.0028 & -0.0013 \\
\hline $\mathrm{CONC}$ & 0.0 & 0.5 & 1.0 & 1.5 & 2.0 & 2.5 & 3.0 \\
\hline ABS at $190 \mathrm{~nm}$ & 0.0002 & 0.0445 & 0.0927 & 0.1456 & 0.1922 & 0.2327 & 0.2891 \\
\hline STDV & 0.0004 & 0.0007 & 0.0038 & 0.0026 & 0.0046 & 0.0223 & 0.0009 \\
\hline
\end{tabular}




\section{Appendix B: UV-vis absorbance data for experimental phase 2}

\begin{tabular}{|c|c|c|c|c|c|c|c|}
\hline \multicolumn{8}{|c|}{ Magnafloc in unfiltered/undiluted Rideau River Water (6.8 NTU) (2018-02-23) } \\
\hline \multicolumn{8}{|c|}{ Desktop spectrophotometer (1 $\mathrm{cm}$ path length) } \\
\hline $\begin{array}{l}\text { Wavelength } \\
(\mathrm{nm})\end{array}$ & $0 \mathrm{mg} / \mathrm{L}$ & $0.5 \mathrm{mg} / \mathrm{L}$ & $1.0 \mathrm{mg} / \mathrm{L}$ & $1.5 \mathrm{mg} / \mathrm{L}$ & $2.0 \mathrm{mg} / \mathrm{L}$ & $2.5 \mathrm{mg} / \mathrm{L}$ & $3.0 \mathrm{mg} / \mathrm{L}$ \\
\hline 250 & 0.2859 & 0.2850 & 0.2872 & 0.2859 & 0.2863 & 0.2866 & 0.2857 \\
\hline 249 & 0.2918 & 0.2907 & 0.2931 & 0.2915 & 0.2920 & 0.2924 & 0.2915 \\
\hline 248 & 0.2977 & 0.2966 & 0.2990 & 0.2975 & 0.2978 & 0.2983 & 0.2973 \\
\hline 247 & 0.3036 & 0.3026 & 0.3048 & 0.3034 & 0.3038 & 0.3042 & 0.3032 \\
\hline 246 & 0.3095 & 0.3084 & 0.3107 & 0.3093 & 0.3095 & 0.3101 & 0.3090 \\
\hline 245 & 0.3153 & 0.3141 & 0.3165 & 0.3149 & 0.3154 & 0.3158 & 0.3148 \\
\hline 244 & 0.3207 & 0.3197 & 0.3221 & 0.3205 & 0.3208 & 0.3212 & 0.3203 \\
\hline 243 & 0.3258 & 0.3249 & 0.3272 & 0.3258 & 0.3260 & 0.3264 & 0.3255 \\
\hline 242 & 0.3307 & 0.3298 & 0.3321 & 0.3307 & 0.3309 & 0.3314 & 0.3303 \\
\hline 241 & 0.3354 & 0.3345 & 0.3367 & 0.3353 & 0.3356 & 0.3359 & 0.3350 \\
\hline 240 & 0.3397 & 0.3388 & 0.3410 & 0.3396 & 0.3400 & 0.3404 & 0.3393 \\
\hline 239 & 0.3439 & 0.3429 & 0.3452 & 0.3437 & 0.3441 & 0.3444 & 0.3435 \\
\hline 238 & 0.3478 & 0.3468 & 0.3491 & 0.3477 & 0.3483 & 0.3485 & 0.3474 \\
\hline 237 & 0.3517 & 0.3507 & 0.3530 & 0.3514 & 0.3520 & 0.3523 & 0.3513 \\
\hline 236 & 0.3554 & 0.3546 & 0.3568 & 0.3553 & 0.3558 & 0.3561 & 0.3552 \\
\hline 235 & 0.3593 & 0.3585 & 0.3607 & 0.3594 & 0.3598 & 0.3600 & 0.3592 \\
\hline 234 & 0.3635 & 0.3627 & 0.3648 & 0.3635 & 0.3639 & 0.3642 & 0.3633 \\
\hline 233 & 0.3681 & 0.3673 & 0.3693 & 0.3680 & 0.3686 & 0.3688 & 0.3680 \\
\hline 232 & 0.3731 & 0.3722 & 0.3744 & 0.3734 & 0.3735 & 0.3738 & 0.3731 \\
\hline 231 & 0.3786 & 0.3777 & 0.3798 & 0.3788 & 0.3791 & 0.3794 & 0.3787 \\
\hline 230 & 0.3847 & 0.3838 & 0.3858 & 0.3850 & 0.3852 & 0.3854 & 0.3849 \\
\hline 229 & 0.3915 & 0.3905 & 0.3928 & 0.3920 & 0.3921 & 0.3924 & 0.3918 \\
\hline 228 & 0.3991 & 0.3979 & 0.4004 & 0.3995 & 0.3998 & 0.3999 & 0.3995 \\
\hline 227 & 0.4076 & 0.4065 & 0.4088 & 0.4081 & 0.4083 & 0.4086 & 0.4081 \\
\hline 226 & 0.4170 & 0.4158 & 0.4183 & 0.4176 & 0.4178 & 0.4180 & 0.4177 \\
\hline 225 & 0.4274 & 0.4263 & 0.4288 & 0.4282 & 0.4284 & 0.4285 & 0.4283 \\
\hline 224 & 0.4391 & 0.4381 & 0.4407 & 0.4400 & 0.4401 & 0.4404 & 0.4401 \\
\hline 223 & 0.4520 & 0.4510 & 0.4536 & 0.4529 & 0.4531 & 0.4534 & 0.4532 \\
\hline 222 & 0.4662 & 0.4652 & 0.4680 & 0.4673 & 0.4675 & 0.4678 & 0.4675 \\
\hline 221 & 0.4818 & 0.4809 & 0.4835 & 0.4830 & 0.4832 & 0.4837 & 0.4833 \\
\hline 220 & 0.4988 & 0.4979 & 0.5006 & 0.5000 & 0.5002 & 0.5006 & 0.5004 \\
\hline 219 & 0.5170 & 0.5163 & 0.5189 & 0.5183 & 0.5188 & 0.5192 & 0.5189 \\
\hline 218 & 0.5366 & 0.5359 & 0.5385 & 0.5379 & 0.5384 & 0.5387 & 0.5388 \\
\hline
\end{tabular}




\begin{tabular}{|c|c|c|c|c|c|c|c|}
\hline 217 & 0.5571 & 0.5563 & 0.5591 & 0.5587 & 0.5594 & 0.5596 & 0.5598 \\
\hline 216 & 0.5787 & 0.5782 & 0.5808 & 0.5804 & 0.5810 & 0.5814 & 0.5815 \\
\hline 215 & 0.6011 & 0.6003 & 0.6033 & 0.6030 & 0.6036 & 0.6040 & 0.6043 \\
\hline 214 & 0.6241 & 0.6236 & 0.6265 & 0.6261 & 0.6267 & 0.6275 & 0.6277 \\
\hline 213 & 0.6478 & 0.6471 & 0.6501 & 0.6501 & 0.6505 & 0.6512 & 0.6519 \\
\hline 212 & 0.6716 & 0.6713 & 0.6741 & 0.6742 & 0.6748 & 0.6755 & 0.6761 \\
\hline 211 & 0.6960 & 0.6955 & 0.6987 & 0.6986 & 0.6993 & 0.7001 & 0.7009 \\
\hline 210 & 0.7205 & 0.7198 & 0.7229 & 0.7232 & 0.7239 & 0.7248 & 0.7258 \\
\hline 209 & 0.7449 & 0.7442 & 0.7476 & 0.7477 & 0.7485 & 0.7494 & 0.7504 \\
\hline 208 & 0.7692 & 0.7684 & 0.7719 & 0.7723 & 0.7733 & 0.7739 & 0.7751 \\
\hline 207 & 0.7934 & 0.7927 & 0.7963 & 0.7968 & 0.7978 & 0.7987 & 0.8002 \\
\hline 206 & 0.8177 & 0.8171 & 0.8208 & 0.8215 & 0.8228 & 0.8234 & 0.8251 \\
\hline 205 & 0.8423 & 0.8417 & 0.8461 & 0.8467 & 0.8480 & 0.8489 & 0.8509 \\
\hline 204 & 0.8678 & 0.8675 & 0.8716 & 0.8728 & 0.8742 & 0.8752 & 0.8775 \\
\hline 203 & 0.8945 & 0.8944 & 0.8990 & 0.9005 & 0.9023 & 0.9035 & 0.9064 \\
\hline 202 & 0.9240 & 0.9239 & 0.9293 & 0.9311 & 0.9333 & 0.9348 & 0.9382 \\
\hline 201 & 0.9564 & 0.9569 & 0.9627 & 0.9652 & 0.9679 & 0.9701 & 0.9737 \\
\hline 200 & 0.9936 & 0.9947 & 1.0011 & 1.0043 & 1.0078 & 1.0104 & 1.0151 \\
\hline 199 & 1.0376 & 1.0394 & 1.0466 & 1.0509 & 1.0548 & 1.0580 & 1.0638 \\
\hline 198 & 1.0896 & 1.0922 & 1.1004 & 1.1053 & 1.1103 & 1.1145 & 1.1212 \\
\hline 197 & 1.1543 & 1.1580 & 1.1672 & 1.1736 & 1.1796 & 1.1847 & 1.1925 \\
\hline 196 & 1.2329 & 1.2380 & 1.2484 & 1.2557 & 1.2632 & 1.2690 & 1.2786 \\
\hline 195 & 1.3309 & 1.3364 & 1.3485 & 1.3568 & 1.3655 & 1.3723 & 1.3835 \\
\hline 194 & 1.4605 & 1.4717 & 1.4810 & 1.4903 & 1.5006 & 1.5085 & 1.5216 \\
\hline 193 & 1.6052 & 1.6130 & 1.6276 & 1.6376 & 1.6491 & 1.6582 & 1.6731 \\
\hline 192 & 1.7922 & 1.8010 & 1.8166 & 1.8272 & 1.8401 & 1.8500 & 1.8655 \\
\hline 191 & 2.0340 & 2.0424 & 2.0587 & 2.0703 & 2.0832 & 2.0943 & 2.1113 \\
\hline 190 & 2.2845 & 2.2933 & 2.3082 & 2.3186 & 2.3315 & 2.3420 & 2.3568 \\
\hline $\mathrm{CONC}$ & 0 & 0.5 & 1 & 1.5 & 2 & 2.5 & 3 \\
\hline ABS at $190 \mathrm{~nm}$ & 2.2845 & 2.2933 & 2.3082 & 2.3186 & 2.3315 & 2.3420 & 2.3568 \\
\hline STDV & 0.0003 & 0.0018 & 0.0014 & 0.0050 & 0.0089 & 0.0083 & 0.0026 \\
\hline
\end{tabular}




\begin{tabular}{|c|c|c|c|c|c|c|c|}
\hline \multicolumn{8}{|c|}{ Magnafloc in unfiltered/undiluted Rideau River water (6.8 NTU) (2018-02-23) } \\
\hline \multicolumn{8}{|c|}{ In-line spectrophotometer (4mm path length) } \\
\hline Wavelength (nm) & $0 \mathrm{mg} / \mathrm{L}$ & $0.5 \mathrm{mg} / \mathrm{L}$ & $1.0 \mathrm{mg} / \mathrm{L}$ & $1.5 \mathrm{mg} / \mathrm{L}$ & $2.0 \mathrm{mg} / \mathrm{L}$ & $2.5 \mathrm{mg} / \mathrm{L}$ & $3.0 \mathrm{mg} / \mathrm{L}$ \\
\hline 189.8 & 1.0473 & 1.0565 & 1.0645 & 1.0750 & 1.0848 & 1.0936 & 1.1019 \\
\hline 192.8 & 0.8462 & 0.8542 & 0.8587 & 0.8659 & 0.8689 & 0.8738 & 0.8792 \\
\hline 195.8 & 0.7659 & 0.7736 & 0.7756 & 0.7807 & 0.7836 & 0.7859 & 0.7888 \\
\hline 198.9 & 0.6971 & 0.7038 & 0.7051 & 0.7073 & 0.7106 & 0.7123 & 0.7140 \\
\hline 201.9 & 0.6445 & 0.6496 & 0.6502 & 0.6528 & 0.6545 & 0.6563 & 0.6581 \\
\hline 204.9 & 0.5843 & 0.5890 & 0.5897 & 0.5923 & 0.5926 & 0.5943 & 0.5955 \\
\hline 208 & 0.5260 & 0.5309 & 0.5314 & 0.5335 & 0.5343 & 0.5351 & 0.5354 \\
\hline 211 & 0.4739 & 0.4787 & 0.4791 & 0.4795 & 0.4811 & 0.4819 & 0.4825 \\
\hline 214 & 0.4301 & 0.4342 & 0.4340 & 0.4347 & 0.4352 & 0.4370 & 0.4374 \\
\hline 217 & 0.3884 & 0.3922 & 0.3921 & 0.3932 & 0.3930 & 0.3940 & 0.3941 \\
\hline 220 & 0.3594 & 0.3637 & 0.3637 & 0.3645 & 0.3650 & 0.3649 & 0.3651 \\
\hline 223.1 & 0.3310 & 0.3355 & 0.3352 & 0.3358 & 0.3366 & 0.3368 & 0.3370 \\
\hline 226.1 & 0.3110 & 0.3151 & 0.3150 & 0.3153 & 0.3152 & 0.3162 & 0.3164 \\
\hline 229.1 & 0.2931 & 0.2970 & 0.2964 & 0.2969 & 0.2969 & 0.2977 & 0.2973 \\
\hline 232.1 & 0.2792 & 0.2826 & 0.2823 & 0.2831 & 0.2838 & 0.2835 & 0.2831 \\
\hline 235.1 & 0.2676 & 0.2718 & 0.2712 & 0.2715 & 0.2724 & 0.2724 & 0.2726 \\
\hline 238.1 & 0.2601 & 0.2601 & 0.2634 & 0.2634 & 0.2632 & 0.2644 & 0.2644 \\
\hline 241.1 & 0.2600 & 0.2562 & 0.2556 & 0.2565 & 0.2559 & 0.2564 & 0.2560 \\
\hline 244.1 & 0.2472 & 0.2503 & 0.2504 & 0.2504 & 0.2512 & 0.2511 & 0.2503 \\
\hline 247.1 & 0.2412 & 0.2452 & 0.2446 & 0.2445 & 0.2454 & 0.2452 & 0.2461 \\
\hline 250.1 & 0.2365 & 0.2399 & 0.2393 & 0.2401 & 0.2396 & 0.2409 & 0.2406 \\
\hline $\mathrm{CONC}$ & 0 & 0.5 & 1 & 1.5 & 2 & 2.5 & 3 \\
\hline ABS AT 190nm & 1.0473 & 1.0565 & 1.0645 & 1.0750 & 1.0848 & 1.0936 & 1.1019 \\
\hline STDV & 0.0021 & 0.0010 & 0.0024 & 0.0021 & 0.0006 & 0.0034 & 0.0013 \\
\hline
\end{tabular}




\begin{tabular}{|c|c|c|c|c|c|c|c|}
\hline \multicolumn{8}{|c|}{ Magnafloc in filtered/undiluted Rideau River water (6.8 NTU) (2018-02-28) } \\
\hline \multicolumn{8}{|c|}{ Desktop spectrophotometer (1 $\mathrm{cm}$ path length) } \\
\hline Wavelength (nm) & $0 \mathrm{mg} / \mathrm{L}$ & $0.5 \mathrm{mg} / \mathrm{L}$ & $1.0 \mathrm{mg} / \mathrm{L}$ & $1.5 \mathrm{mg} / \mathrm{L}$ & $2.0 \mathrm{mg} / \mathrm{L}$ & $2.5 \mathrm{mg} / \mathrm{L}$ & $3.0 \mathrm{mg} / \mathrm{L}$ \\
\hline 250 & 0.2503 & 0.2496 & 0.2445 & 0.2449 & 0.2436 & 0.2416 & 0.2408 \\
\hline 249 & 0.2561 & 0.2554 & 0.2503 & 0.2505 & 0.2493 & 0.2472 & 0.2464 \\
\hline 248 & 0.2618 & 0.2610 & 0.2558 & 0.2561 & 0.2548 & 0.2529 & 0.2519 \\
\hline 247 & 0.2678 & 0.2668 & 0.2617 & 0.2620 & 0.2607 & 0.2586 & 0.2578 \\
\hline 246 & 0.2735 & 0.2726 & 0.2673 & 0.2679 & 0.2663 & 0.2643 & 0.2635 \\
\hline 245 & 0.2793 & 0.2781 & 0.2730 & 0.2734 & 0.2721 & 0.2699 & 0.2691 \\
\hline 244 & 0.2846 & 0.2835 & 0.2783 & 0.2787 & 0.2772 & 0.2751 & 0.2743 \\
\hline 243 & 0.2897 & 0.2887 & 0.2833 & 0.2838 & 0.2823 & 0.2802 & 0.2794 \\
\hline 242 & 0.2946 & 0.2935 & 0.2880 & 0.2883 & 0.2871 & 0.2849 & 0.2840 \\
\hline 241 & 0.2991 & 0.2980 & 0.2925 & 0.2928 & 0.2914 & 0.2892 & 0.2884 \\
\hline 240 & 0.3033 & 0.3021 & 0.2967 & 0.2969 & 0.2955 & 0.2933 & 0.2925 \\
\hline 239 & 0.3073 & 0.3061 & 0.3005 & 0.3009 & 0.2995 & 0.2973 & 0.2964 \\
\hline 238 & 0.3112 & 0.3099 & 0.3042 & 0.3045 & 0.3032 & 0.3009 & 0.2999 \\
\hline 237 & 0.3150 & 0.3136 & 0.3079 & 0.3082 & 0.3069 & 0.3045 & 0.3036 \\
\hline 236 & 0.3187 & 0.3175 & 0.3118 & 0.3119 & 0.3107 & 0.3082 & 0.3073 \\
\hline 235 & 0.3228 & 0.3214 & 0.3155 & 0.3158 & 0.3145 & 0.3121 & 0.3112 \\
\hline 234 & 0.3270 & 0.3257 & 0.3198 & 0.3200 & 0.3187 & 0.3162 & 0.3154 \\
\hline 233 & 0.3319 & 0.3304 & 0.3244 & 0.3249 & 0.3233 & 0.3209 & 0.3200 \\
\hline 232 & 0.3371 & 0.3356 & 0.3295 & 0.3301 & 0.3287 & 0.3259 & 0.3252 \\
\hline 231 & 0.3429 & 0.3414 & 0.3350 & 0.3357 & 0.3344 & 0.3317 & 0.3309 \\
\hline 230 & 0.3493 & 0.3479 & 0.3415 & 0.3420 & 0.3407 & 0.3378 & 0.3372 \\
\hline 229 & 0.3565 & 0.3548 & 0.3483 & 0.3490 & 0.3478 & 0.3449 & 0.3442 \\
\hline 228 & 0.3643 & 0.3627 & 0.3561 & 0.3567 & 0.3554 & 0.3526 & 0.3520 \\
\hline 227 & 0.3730 & 0.3714 & 0.3647 & 0.3654 & 0.3642 & 0.3613 & 0.3607 \\
\hline 226 & 0.3827 & 0.3809 & 0.3744 & 0.3750 & 0.3738 & 0.3708 & 0.3702 \\
\hline 225 & 0.3935 & 0.3919 & 0.3851 & 0.3856 & 0.3845 & 0.3816 & 0.3811 \\
\hline 224 & 0.4053 & 0.4035 & 0.3966 & 0.3974 & 0.3962 & 0.3932 & 0.3927 \\
\hline 223 & 0.4185 & 0.4166 & 0.4097 & 0.4103 & 0.4092 & 0.4062 & 0.4059 \\
\hline 222 & 0.4326 & 0.4308 & 0.4236 & 0.4244 & 0.4234 & 0.4202 & 0.4198 \\
\hline 221 & 0.4483 & 0.4465 & 0.4392 & 0.4401 & 0.4391 & 0.4360 & 0.4354 \\
\hline 220 & 0.4651 & 0.4633 & 0.4558 & 0.4568 & 0.4557 & 0.4526 & 0.4522 \\
\hline 219 & 0.4836 & 0.4818 & 0.4741 & 0.4752 & 0.4741 & 0.4711 & 0.4707 \\
\hline 218 & 0.5029 & 0.5010 & 0.4933 & 0.4945 & 0.4935 & 0.4903 & 0.4899 \\
\hline 217 & 0.5237 & 0.5218 & 0.5138 & 0.5152 & 0.5144 & 0.5113 & 0.5108 \\
\hline 216 & 0.5452 & 0.5432 & 0.5349 & 0.5364 & 0.5357 & 0.5325 & 0.5319 \\
\hline 215 & 0.5679 & 0.5657 & 0.5574 & 0.5590 & 0.5583 & 0.5551 & 0.5547 \\
\hline
\end{tabular}




\begin{tabular}{|c|c|c|c|c|c|c|c|}
\hline 214 & 0.5909 & 0.5887 & 0.5801 & 0.5819 & 0.5812 & 0.5781 & 0.5777 \\
\hline 213 & 0.6151 & 0.6127 & 0.6037 & 0.6059 & 0.6051 & 0.6019 & 0.6016 \\
\hline 212 & 0.6389 & 0.6365 & 0.6274 & 0.6297 & 0.6290 & 0.6260 & 0.6257 \\
\hline 211 & 0.6635 & 0.6611 & 0.6515 & 0.6541 & 0.6538 & 0.6505 & 0.6502 \\
\hline 210 & 0.6879 & 0.6853 & 0.6755 & 0.6783 & 0.6779 & 0.6748 & 0.6744 \\
\hline 209 & 0.7125 & 0.7098 & 0.6997 & 0.7030 & 0.7025 & 0.6994 & 0.6991 \\
\hline 208 & 0.7368 & 0.7339 & 0.7235 & 0.7269 & 0.7266 & 0.7235 & 0.7232 \\
\hline 207 & 0.7613 & 0.7583 & 0.7474 & 0.7513 & 0.7511 & 0.7481 & 0.7480 \\
\hline 206 & 0.7856 & 0.7822 & 0.7710 & 0.7753 & 0.7753 & 0.7722 & 0.7724 \\
\hline 205 & 0.8106 & 0.8070 & 0.7954 & 0.8001 & 0.8005 & 0.7974 & 0.7977 \\
\hline 204 & 0.8360 & 0.8323 & 0.8199 & 0.8253 & 0.8262 & 0.8234 & 0.8240 \\
\hline 203 & 0.8632 & 0.8592 & 0.8465 & 0.8528 & 0.8541 & 0.8515 & 0.8525 \\
\hline 202 & 0.8927 & 0.8884 & 0.8752 & 0.8828 & 0.8850 & 0.8823 & 0.8841 \\
\hline 201 & 0.9263 & 0.9218 & 0.9079 & 0.9169 & 0.9198 & 0.9176 & 0.9203 \\
\hline 200 & 0.9644 & 0.9596 & 0.9447 & 0.9555 & 0.9597 & 0.9579 & 0.9616 \\
\hline 199 & 1.0094 & 1.0046 & 0.9885 & 1.0015 & 1.0071 & 1.0059 & 1.0109 \\
\hline 198 & 1.0613 & 1.0564 & 1.0393 & 1.0550 & 1.0617 & 1.0613 & 1.0681 \\
\hline 197 & 1.1254 & 1.1198 & 1.1014 & 1.1197 & 1.1283 & 1.1288 & 1.1377 \\
\hline 196 & 1.2006 & 1.1944 & 1.1748 & 1.1961 & 1.2064 & 1.2083 & 1.2193 \\
\hline 195 & 1.2934 & 1.2858 & 1.2655 & 1.2895 & 1.3020 & 1.3054 & 1.3189 \\
\hline 194 & 1.4185 & 1.4093 & 1.3877 & 1.4144 & 1.4294 & 1.4341 & 1.4509 \\
\hline 193 & 1.5506 & 1.5392 & 1.5175 & 1.5464 & 1.5632 & 1.5697 & 1.5893 \\
\hline 192 & 1.7258 & 1.7118 & 1.6897 & 1.7193 & 1.7387 & 1.7465 & 1.7692 \\
\hline 191 & 1.9528 & 1.9356 & 1.9134 & 1.9436 & 1.9644 & 1.9738 & 1.9990 \\
\hline 190 & 2.1886 & 2.1694 & 2.1473 & 2.1758 & 2.1988 & 2.2060 & 2.2324 \\
\hline $\mathrm{CONC}$ & 0 & 0.5 & 1 & 1.5 & 2 & 2.5 & 3 \\
\hline ABS at $190 \mathrm{~nm}$ & 2.1886 & 2.1694 & 2.1473 & 2.1758 & 2.1988 & 2.2060 & 2.2324 \\
\hline STDV & 0.0494 & 0.0518 & 0.0432 & 0.0089 & 0.0219 & 0.0291 & 0.0153 \\
\hline
\end{tabular}




\begin{tabular}{|c|c|c|c|c|c|c|c|}
\hline \multicolumn{8}{|c|}{ Magnafloc in filtered/undiluted Rideau River water (6.8 NTU) (2018-02-28) } \\
\hline \multicolumn{8}{|c|}{ In-line spectrophotometer (4mm path length) } \\
\hline Wavelength (nm) & $0 \mathrm{mg} / \mathrm{L}$ & $0.5 \mathrm{mg} / \mathrm{L}$ & $1.0 \mathrm{mg} / \mathrm{L}$ & $1.5 \mathrm{mg} / \mathrm{L}$ & $2.0 \mathrm{mg} / \mathrm{L}$ & $2.5 \mathrm{mg} / \mathrm{L}$ & $3.0 \mathrm{mg} / \mathrm{L}$ \\
\hline 189.8 & 0.9671 & 0.9759 & 1.1271 & 1.1632 & 1.1786 & 1.1918 & 1.2087 \\
\hline 192.8 & 0.7810 & 0.7881 & 0.8619 & 0.8801 & 0.8829 & 0.8947 & 0.9038 \\
\hline 195.8 & 0.7037 & 0.7072 & 0.7452 & 0.7561 & 0.7557 & 0.7631 & 0.7688 \\
\hline 198.9 & 0.6391 & 0.6419 & 0.6742 & 0.6808 & 0.6789 & 0.6845 & 0.6872 \\
\hline 201.9 & 0.5905 & 0.5946 & 0.6183 & 0.6216 & 0.6177 & 0.6233 & 0.6255 \\
\hline 204.9 & 0.5333 & 0.5362 & 0.5644 & 0.5659 & 0.5631 & 0.5667 & 0.5692 \\
\hline 208 & 0.4767 & 0.4785 & 0.5086 & 0.5108 & 0.5069 & 0.5110 & 0.5121 \\
\hline 211 & 0.4258 & 0.4286 & 0.4582 & 0.4587 & 0.4809 & 0.4589 & 0.4591 \\
\hline 214 & 0.3828 & 0.3856 & 0.4087 & 0.4100 & 0.4073 & 0.4094 & 0.4107 \\
\hline 217 & 0.3427 & 0.3443 & 0.3659 & 0.3661 & 0.3626 & 0.3654 & 0.3655 \\
\hline 220 & 0.3138 & 0.3160 & 0.3309 & 0.3301 & 0.3273 & 0.3297 & 0.3301 \\
\hline 223.1 & 0.2846 & 0.2871 & 0.3009 & 0.3010 & 0.2988 & 0.2999 & 0.3004 \\
\hline 226.1 & 0.2644 & 0.2658 & 0.2760 & 0.2757 & 0.2724 & 0.2747 & 0.2743 \\
\hline 229.1 & 0.2469 & 0.2491 & 0.2576 & 0.2570 & 0.2543 & 0.2556 & 0.2556 \\
\hline 232.1 & 0.2333 & 0.2351 & 0.2417 & 0.2416 & 0.2394 & 0.2399 & 0.2404 \\
\hline 235.1 & 0.2227 & 0.2241 & 0.2307 & 0.2304 & 0.2272 & 0.2291 & 0.2283 \\
\hline 238.1 & 0.2159 & 0.2174 & 0.2218 & 0.2211 & 0.2188 & 0.2198 & 0.2199 \\
\hline 241.1 & 0.2091 & 0.2106 & 0.2138 & 0.2139 & 0.2116 & 0.2124 & 0.2130 \\
\hline 244.1 & 0.2042 & 0.2051 & 0.2085 & 0.2085 & 0.2052 & 0.2069 & 0.2065 \\
\hline 247.1 & 0.1990 & 0.1999 & 0.2036 & 0.2030 & 0.2011 & 0.2015 & 0.2018 \\
\hline 250.1 & 0.1950 & 0.1965 & 0.1988 & 0.1988 & 0.1962 & 0.1972 & 0.1975 \\
\hline CONC & 0 & 0.5 & 1 & 1.5 & 2 & 2.5 & 3 \\
\hline ABS at $190 \mathrm{~nm}$ & 0.9671 & 0.9759 & 1.1271 & 1.1632 & 1.1786 & 1.1918 & 1.2087 \\
\hline STDV & 0.0025 & 0.0041 & 0.0031 & 0.0043 & 0.0037 & 0.0055 & 0.0020 \\
\hline
\end{tabular}




\begin{tabular}{|c|c|c|c|c|c|c|c|}
\hline \multicolumn{8}{|c|}{ Magnafloc in unfiltered/diluted Rideau River water (6.8 NTU) (2018-02-23) } \\
\hline \multicolumn{8}{|c|}{ In-line spectrophotometer (4mm path length) } \\
\hline Wavelength (nm) & $0 \mathrm{mg} / \mathrm{L}$ & $0.5 \mathrm{mg} / \mathrm{L}$ & $1.0 \mathrm{mg} / \mathrm{L}$ & $1.5 \mathrm{mg} / \mathrm{L}$ & $2.0 \mathrm{mg} / \mathrm{L}$ & $2.5 \mathrm{mg} / \mathrm{L}$ & $3.0 \mathrm{mg} / \mathrm{L}$ \\
\hline 189.8 & 0.5153 & 0.5221 & 0.5258 & 0.5336 & 0.5353 & 0.5403 & 0.5464 \\
\hline 192.8 & 0.4212 & 0.4259 & 0.4280 & 0.4322 & 0.4339 & 0.4357 & 0.4383 \\
\hline 195.8 & 0.3818 & 0.3856 & 0.3881 & 0.3907 & 0.3921 & 0.3943 & 0.3963 \\
\hline 198.9 & 0.3473 & 0.3510 & 0.3530 & 0.3560 & 0.3556 & 0.3556 & 0.3588 \\
\hline 201.9 & 0.3210 & 0.3250 & 0.3253 & 0.3279 & 0.3281 & 0.3283 & 0.3298 \\
\hline 204.9 & 0.2933 & 0.2968 & 0.2971 & 0.2992 & 0.2995 & 0.2994 & 0.3006 \\
\hline 208 & 0.2639 & 0.2666 & 0.2680 & 0.2697 & 0.2699 & 0.2688 & 0.2716 \\
\hline 211 & 0.2370 & 0.2403 & 0.2411 & 0.2427 & 0.2421 & 0.2422 & 0.2438 \\
\hline 214 & 0.2190 & 0.2179 & 0.2183 & 0.2197 & 0.2200 & 0.2196 & 0.2202 \\
\hline 217 & 0.1947 & 0.1978 & 0.1979 & 0.1995 & 0.1994 & 0.1985 & 0.1998 \\
\hline 220 & 0.1808 & 0.1829 & 0.1838 & 0.1851 & 0.1844 & 0.1840 & 0.1859 \\
\hline 223.1 & 0.1648 & 0.1675 & 0.1680 & 0.1699 & 0.1692 & 0.1690 & 0.1693 \\
\hline 226.1 & 0.1551 & 0.1574 & 0.1576 & 0.1589 & 0.1592 & 0.1585 & 0.1592 \\
\hline 229.1 & 0.1465 & 0.1494 & 0.1495 & 0.1507 & 0.1503 & 0.1497 & 0.1511 \\
\hline 232.1 & 0.1396 & 0.1419 & 0.1428 & 0.1440 & 0.1434 & 0.1428 & 0.1438 \\
\hline 235.1 & 0.1328 & 0.1354 & 0.1360 & 0.1370 & 0.1370 & 0.1373 & 0.1367 \\
\hline 238.1 & 0.1288 & 0.1321 & 0.1313 & 0.1323 & 0.1325 & 0.1321 & 0.1330 \\
\hline 241.1 & 0.1260 & 0.1288 & 0.1283 & 0.1293 & 0.1294 & 0.1285 & 0.1301 \\
\hline 244.1 & 0.1237 & 0.1255 & 0.1267 & 0.1274 & 0.1271 & 0.1269 & 0.1273 \\
\hline 247.1 & 0.1205 & 0.1211 & 0.1221 & 0.1231 & 0.1227 & 0.1229 & 0.1235 \\
\hline 250.1 & 0.1169 & 0.1197 & 0.1187 & 0.1200 & 0.1202 & 0.1195 & 0.1210 \\
\hline CONC & 0 & 0.5 & 1 & 1.5 & 2 & 2.5 & 3 \\
\hline $\mathrm{ABS}$ at $190 \mathrm{NM}$ & 0.5153 & 0.5221 & 0.5258 & 0.5336 & 0.5353 & 0.5403 & 0.5464 \\
\hline STDV & 0.0003 & 0.0000 & 0.0000 & 0.0009 & 0.0000 & 0.0011 & 0.0006 \\
\hline
\end{tabular}




\begin{tabular}{|c|c|c|c|c|c|c|c|}
\hline \multicolumn{8}{|c|}{ Magnafloc in filtered/diluted Rideau River water (6.8 NTU) (2018-02-28) } \\
\hline \multicolumn{8}{|c|}{ In-line spectrophotometer (4mm path length) } \\
\hline Wavelength (nm) & $0 \mathrm{mg} / \mathrm{L}$ & $0.5 \mathrm{mg} / \mathrm{L}$ & $1.0 \mathrm{mg} / \mathrm{L}$ & $1.5 \mathrm{mg} / \mathrm{L}$ & $2.0 \mathrm{mg} / \mathrm{L}$ & $2.5 \mathrm{mg} / \mathrm{L}$ & $3.0 \mathrm{mg} / \mathrm{L}$ \\
\hline 189.8 & 0.5658 & 0.5761 & 0.5745 & 0.5860 & 0.5850 & 0.5942 & 0.6042 \\
\hline 192.8 & 0.4260 & 0.4339 & 0.4320 & 0.4395 & 0.4373 & 0.4423 & 0.4488 \\
\hline 195.8 & 0.3643 & 0.3695 & 0.3690 & 0.3731 & 0.3700 & 0.3737 & 0.3791 \\
\hline 198.9 & 0.3282 & 0.3331 & 0.3329 & 0.3345 & 0.3317 & 0.3338 & 0.3381 \\
\hline 201.9 & 0.2997 & 0.3036 & 0.3035 & 0.3056 & 0.3020 & 0.3038 & 0.3117 \\
\hline 204.9 & 0.2737 & 0.2760 & 0.2772 & 0.2777 & 0.2744 & 0.2756 & 0.2794 \\
\hline 208 & 0.2458 & 0.2497 & 0.2498 & 0.2501 & 0.2471 & 0.2480 & 0.2513 \\
\hline 211 & 0.2201 & 0.2234 & 0.2239 & 0.2242 & 0.2215 & 0.2220 & 0.2250 \\
\hline 214 & 0.1966 & 0.1990 & 0.1999 & 0.1999 & 0.1969 & 0.1972 & 0.2004 \\
\hline 217 & 0.1751 & 0.1782 & 0.1788 & 0.1787 & 0.1761 & 0.1765 & 0.1787 \\
\hline 220 & 0.1572 & 0.1594 & 0.1604 & 0.1607 & 0.1580 & 0.1577 & 0.1603 \\
\hline 223.1 & 0.1428 & 0.1453 & 0.1460 & 0.1456 & 0.1429 & 0.1432 & 0.1455 \\
\hline 226.1 & 0.1301 & 0.1328 & 0.1335 & 0.1333 & 0.1310 & 0.1308 & 0.1330 \\
\hline 229.1 & 0.1213 & 0.1233 & 0.1243 & 0.1241 & 0.1221 & 0.1215 & 0.1239 \\
\hline 232.1 & 0.1139 & 0.1165 & 0.1166 & 0.1167 & 0.1143 & 0.1142 & 0.1162 \\
\hline 235.1 & 0.1079 & 0.1100 & 0.1108 & 0.1107 & 0.1090 & 0.1086 & 0.1102 \\
\hline 238.1 & 0.1043 & 0.1057 & 0.1067 & 0.1066 & 0.1047 & 0.1040 & 0.1061 \\
\hline 241.1 & 0.1004 & 0.1033 & 0.1034 & 0.1032 & 0.1010 & 0.1010 & 0.1028 \\
\hline 244.1 & 0.0975 & 0.0994 & 0.1002 & 0.1003 & 0.0985 & 0.0981 & 0.0997 \\
\hline 247.1 & 0.0953 & 0.0969 & 0.0976 & 0.0977 & 0.0958 & 0.0951 & 0.0977 \\
\hline 250.1 & 0.0933 & 0.0955 & 0.0959 & 0.0958 & 0.0938 & 0.0939 & 0.0954 \\
\hline CONC & 0 & 0.5 & 1 & 1.5 & 2 & 2.5 & 3 \\
\hline ABS at $190 \mathrm{~nm}$ & 0.5658 & 0.5761 & 0.5745 & 0.5860 & 0.5850 & 0.5942 & 0.6042 \\
\hline STDV & 0.0014 & 0.0016 & 0.0013 & 0.0010 & 0.0007 & 0.0018 & 0.0024 \\
\hline
\end{tabular}




\begin{tabular}{|c|c|c|c|c|c|c|c|c|c|c|c|}
\hline \multicolumn{12}{|c|}{ Magnafloc in unfiltered/undiluted Rideau River water (3.2 NTU) (2018-03-21) } \\
\hline \multicolumn{12}{|c|}{ Desktop spectrophotometer (1 cm path length) } \\
\hline $\begin{array}{l}\text { Wavelength } \\
(\mathrm{nm})\end{array}$ & $0 \mathrm{mg} / \mathrm{L}$ & $\begin{array}{c}0.1 \\
\mathrm{mg} / \mathrm{L}\end{array}$ & $\begin{array}{c}0.2 \\
\mathrm{mg} / \mathrm{L}\end{array}$ & $\begin{array}{c}0.3 \\
\mathrm{mg} / \mathrm{L}\end{array}$ & $\begin{array}{c}0.4 \\
\mathrm{mg} / \mathrm{L}\end{array}$ & $\begin{array}{c}0.5 \\
\mathrm{mg} / \mathrm{L}\end{array}$ & $\begin{array}{c}0.6 \\
\mathrm{mg} / \mathrm{L}\end{array}$ & $\begin{array}{c}0.7 \\
\mathrm{mg} / \mathrm{L}\end{array}$ & $\begin{array}{c}0.8 \\
\mathrm{mg} / \mathrm{L}\end{array}$ & $\begin{array}{c}0.9 \\
\mathrm{mg} / \mathrm{L}\end{array}$ & $\begin{array}{c}1.0 \\
\mathrm{mg} / \mathrm{L}\end{array}$ \\
\hline 250 & 0.2567 & 0.2649 & 0.2618 & 0.2642 & 0.2655 & 0.2643 & 0.2655 & 0.2669 & 0.2667 & 0.2676 & 0.2674 \\
\hline 249 & 0.2624 & 0.2708 & 0.2676 & 0.2699 & 0.2713 & 0.2700 & 0.2712 & 0.2729 & 0.2725 & 0.2737 & 0.2734 \\
\hline 248 & 0.2683 & 0.2765 & 0.2736 & 0.2759 & 0.2772 & 0.2759 & 0.2771 & 0.2788 & 0.2783 & 0.2795 & 0.2792 \\
\hline 247 & 0.2741 & 0.2823 & 0.2794 & 0.2817 & 0.2831 & 0.2819 & 0.2831 & 0.2847 & 0.2845 & 0.2854 & 0.2851 \\
\hline 246 & 0.2798 & 0.2880 & 0.2852 & 0.2875 & 0.2888 & 0.2876 & 0.2888 & 0.2904 & 0.2902 & 0.2912 & 0.2910 \\
\hline 245 & 0.2854 & 0.2938 & 0.2909 & 0.2932 & 0.2946 & 0.2934 & 0.2944 & 0.2963 & 0.2961 & 0.2971 & 0.2968 \\
\hline 244 & 0.2908 & 0.2991 & 0.2963 & 0.2986 & 0.3001 & 0.2989 & 0.3001 & 0.3018 & 0.3013 & 0.3024 & 0.3022 \\
\hline 243 & 0.2958 & 0.3043 & 0.3015 & 0.3036 & 0.3051 & 0.3040 & 0.3051 & 0.3070 & 0.3066 & 0.3077 & 0.3074 \\
\hline 242 & 0.3005 & 0.3091 & 0.3062 & 0.3084 & 0.3099 & 0.3087 & 0.3102 & 0.3118 & 0.3114 & 0.3126 & 0.3122 \\
\hline 241 & 0.3048 & 0.3134 & 0.3106 & 0.3128 & 0.3144 & 0.3132 & 0.3145 & 0.3163 & 0.3159 & 0.3171 & 0.3167 \\
\hline 240 & 0.3088 & 0.3176 & 0.3146 & 0.3170 & 0.3187 & 0.3172 & 0.3188 & 0.3205 & 0.3202 & 0.3212 & 0.3210 \\
\hline 239 & 0.3126 & 0.3215 & 0.3185 & 0.3211 & 0.3225 & 0.3214 & 0.3227 & 0.3245 & 0.3241 & 0.3251 & 0.3250 \\
\hline 238 & 0.3161 & 0.3252 & 0.3221 & 0.3245 & 0.3263 & 0.3250 & 0.3264 & 0.3282 & 0.3279 & 0.3289 & 0.3287 \\
\hline 237 & 0.3196 & 0.3287 & 0.3256 & 0.3282 & 0.3298 & 0.3285 & 0.3301 & 0.3318 & 0.3315 & 0.3324 & 0.3324 \\
\hline 236 & 0.3230 & 0.3322 & 0.3292 & 0.3316 & 0.3332 & 0.3320 & 0.3336 & 0.3354 & 0.3351 & 0.3362 & 0.3360 \\
\hline 235 & 0.3266 & 0.3358 & 0.3326 & 0.3353 & 0.3370 & 0.3356 & 0.3371 & 0.3390 & 0.3386 & 0.3397 & 0.3396 \\
\hline 234 & 0.3301 & 0.3396 & 0.3364 & 0.3391 & 0.3407 & 0.3393 & 0.3409 & 0.3428 & 0.3424 & 0.3435 & 0.3434 \\
\hline 233 & 0.3342 & 0.3437 & 0.3404 & 0.3432 & 0.3448 & 0.3433 & 0.3451 & 0.3470 & 0.3467 & 0.3478 & 0.3476 \\
\hline 232 & 0.3386 & 0.3483 & 0.3449 & 0.3479 & 0.3493 & 0.3478 & 0.3497 & 0.3516 & 0.3513 & 0.3523 & 0.3523 \\
\hline 231 & 0.3434 & 0.3535 & 0.3498 & 0.3528 & 0.3543 & 0.3530 & 0.3547 & 0.3567 & 0.3563 & 0.3575 & 0.3573 \\
\hline 230 & 0.3489 & 0.3590 & 0.3554 & 0.3585 & 0.3600 & 0.3585 & 0.3604 & 0.3623 & 0.3620 & 0.3633 & 0.3630 \\
\hline 229 & 0.3550 & 0.3651 & 0.3615 & 0.3648 & 0.3663 & 0.3647 & 0.3668 & 0.3688 & 0.3685 & 0.3698 & 0.3694 \\
\hline 228 & 0.3619 & 0.3723 & 0.3687 & 0.3720 & 0.3736 & 0.3719 & 0.3740 & 0.3761 & 0.3757 & 0.3771 & 0.3766 \\
\hline 227 & 0.3696 & 0.3803 & 0.3766 & 0.3798 & 0.3814 & 0.3799 & 0.3821 & 0.3843 & 0.3838 & 0.3852 & 0.3847 \\
\hline 226 & 0.3785 & 0.3893 & 0.3855 & 0.3889 & 0.3905 & 0.3890 & 0.3912 & 0.3931 & 0.3928 & 0.3944 & 0.3939 \\
\hline 225 & 0.3883 & 0.3993 & 0.3952 & 0.3988 & 0.4004 & 0.3989 & 0.4011 & 0.4034 & 0.4029 & 0.4046 & 0.4040 \\
\hline 224 & 0.3991 & 0.4104 & 0.4065 & 0.4101 & 0.4118 & 0.4102 & 0.4125 & 0.4147 & 0.4143 & 0.4160 & 0.4154 \\
\hline 223 & 0.4112 & 0.4229 & 0.4188 & 0.4225 & 0.4241 & 0.4226 & 0.4250 & 0.4272 & 0.4270 & 0.4286 & 0.4281 \\
\hline 222 & 0.4248 & 0.4368 & 0.4324 & 0.4363 & 0.4381 & 0.4364 & 0.4390 & 0.4413 & 0.4409 & 0.4428 & 0.4423 \\
\hline 221 & 0.4396 & 0.4518 & 0.4475 & 0.4513 & 0.4532 & 0.4516 & 0.4541 & 0.4564 & 0.4560 & 0.4580 & 0.4574 \\
\hline 220 & 0.4557 & 0.4684 & 0.4638 & 0.4680 & 0.4698 & 0.4683 & 0.4709 & 0.4732 & 0.4729 & 0.4747 & 0.4743 \\
\hline 219 & 0.4731 & 0.4861 & 0.4814 & 0.4858 & 0.4876 & 0.4860 & 0.4888 & 0.4910 & 0.4908 & 0.4928 & 0.4924 \\
\hline 218 & 0.4920 & 0.5053 & 0.5005 & 0.5050 & 0.5070 & 0.5053 & 0.5082 & 0.5106 & 0.5104 & 0.5123 & 0.5121 \\
\hline 217 & 0.5117 & 0.5256 & 0.5207 & 0.5253 & 0.5273 & 0.5256 & 0.5285 & 0.5309 & 0.5308 & 0.5329 & 0.5326 \\
\hline 216 & 0.5329 & 0.5470 & 0.5418 & 0.5466 & 0.5488 & 0.5471 & 0.5500 & 0.5525 & 0.5524 & 0.5544 & 0.5544 \\
\hline
\end{tabular}




\begin{tabular}{|c|c|c|c|c|c|c|c|c|c|c|c|}
\hline 215 & 0.5543 & 0.5690 & 0.5637 & 0.5687 & 0.5709 & 0.5692 & 0.5721 & 0.5748 & 0.5747 & 0.5769 & 0.5769 \\
\hline 214 & 0.5770 & 0.5919 & 0.5863 & 0.5918 & 0.5941 & 0.5922 & 0.5954 & 0.5980 & 0.5981 & 0.6001 & 0.6001 \\
\hline 213 & 0.5996 & 0.6150 & 0.6095 & 0.6151 & 0.6175 & 0.6157 & 0.6187 & 0.6217 & 0.6216 & 0.6238 & 0.6240 \\
\hline 212 & 0.6230 & 0.6389 & 0.6330 & 0.6389 & 0.6414 & 0.6397 & 0.6427 & 0.6457 & 0.6457 & 0.6480 & 0.6484 \\
\hline 211 & 0.6465 & 0.6626 & 0.6566 & 0.6629 & 0.6653 & 0.6637 & 0.6668 & 0.6698 & 0.6701 & 0.6725 & 0.6729 \\
\hline 210 & 0.6701 & 0.6867 & 0.6806 & 0.6871 & 0.6897 & 0.6878 & 0.6910 & 0.6942 & 0.6945 & 0.6969 & 0.6976 \\
\hline 209 & 0.6937 & 0.7105 & 0.7043 & 0.7111 & 0.7138 & 0.7120 & 0.7153 & 0.7187 & 0.7187 & 0.7214 & 0.7219 \\
\hline 208 & 0.7170 & 0.7342 & 0.7278 & 0.7347 & 0.7376 & 0.7359 & 0.7394 & 0.7428 & 0.7431 & 0.7456 & 0.7464 \\
\hline 207 & 0.7402 & 0.7577 & 0.7509 & 0.7583 & 0.7616 & 0.7597 & 0.7632 & 0.7668 & 0.7671 & 0.7701 & 0.7707 \\
\hline 206 & 0.7633 & 0.7811 & 0.7741 & 0.7816 & 0.7853 & 0.7834 & 0.7870 & 0.7908 & 0.7913 & 0.7943 & 0.7951 \\
\hline 205 & 0.7866 & 0.8047 & 0.7972 & 0.8053 & 0.8092 & 0.8073 & 0.8111 & 0.8151 & 0.8156 & 0.8189 & 0.8195 \\
\hline 204 & 0.8107 & 0.8291 & 0.8211 & 0.8298 & 0.8338 & 0.8319 & 0.8362 & 0.8402 & 0.8410 & 0.8445 & 0.8450 \\
\hline 203 & 0.8352 & 0.8539 & 0.8455 & 0.8547 & 0.8592 & 0.8571 & 0.8618 & 0.8661 & 0.8671 & 0.8709 & 0.8712 \\
\hline 202 & 0.8614 & 0.8805 & 0.8717 & 0.8814 & 0.8865 & 0.8843 & 0.8892 & 0.8939 & 0.8950 & 0.8993 & 0.8998 \\
\hline 201 & 0.8899 & 0.9095 & 0.9001 & 0.9108 & 0.9160 & 0.9137 & 0.9193 & 0.9241 & 0.9260 & 0.9304 & 0.9310 \\
\hline 200 & 0.9222 & 0.9423 & 0.9322 & 0.9438 & 0.9498 & 0.9472 & 0.9530 & 0.9584 & 0.9606 & 0.9655 & 0.9660 \\
\hline 199 & 0.9591 & 0.9802 & 0.9691 & 0.9816 & 0.9882 & 0.9851 & 0.9924 & 0.9976 & 1.0004 & 1.0057 & 1.0066 \\
\hline 198 & 1.0019 & 1.0236 & 1.0117 & 1.0254 & 1.0330 & 1.0296 & 1.0374 & 1.0432 & 1.0468 & 1.0527 & 1.0533 \\
\hline 197 & 1.0536 & 1.0762 & 1.0633 & 1.0784 & 1.0866 & 1.0825 & 1.0920 & 1.0974 & 1.1022 & 1.1091 & 1.1117 \\
\hline 196 & 1.1155 & 1.1392 & 1.1251 & 1.1415 & 1.1507 & 1.1460 & 1.1564 & 1.1626 & 1.1684 & 1.1763 & 1.1766 \\
\hline 195 & 1.1935 & 1.2182 & 1.2027 & 1.2206 & 1.2314 & 1.2255 & 1.2380 & 1.2441 & 1.2514 & 1.2607 & 1.2590 \\
\hline 194 & 1.2841 & 1.3104 & 1.2931 & 1.3129 & 1.3246 & 1.3180 & 1.3321 & 1.3387 & 1.3478 & 1.3578 & 1.3581 \\
\hline 193 & 1.3952 & 1.4227 & 1.4042 & 1.4254 & 1.4389 & 1.4302 & 1.4471 & 1.4533 & 1.4650 & 1.4761 & 1.4765 \\
\hline 192 & 1.5364 & 1.5665 & 1.5449 & 1.5684 & 1.5841 & 1.5738 & 1.5930 & 1.5990 & 1.6128 & 1.6263 & 1.6258 \\
\hline 191 & 1.7201 & 1.7516 & 1.7278 & 1.7527 & 1.7705 & 1.7585 & 1.7809 & 1.7865 & 1.8026 & 1.8173 & 1.8210 \\
\hline 190 & 1.9261 & 1.9586 & 1.9328 & 1.9600 & 1.9785 & 1.9637 & 1.9891 & 1.9946 & 2.0129 & 2.0276 & 2.0232 \\
\hline $\mathrm{CONC}$ & 0 & 0.1 & 0.2 & 0.3 & 0.4 & 0.5 & 0.6 & 0.7 & 0.8 & 0.9 & 1 \\
\hline $\begin{array}{l}\text { ABS at } \\
190 \mathrm{~nm}\end{array}$ & 1.9261 & 1.9586 & 1.9328 & 1.9600 & 1.9785 & 1.9637 & 1.9891 & 1.9946 & 2.0129 & 2.0276 & 2.0232 \\
\hline STDV & 0.0145 & 0.0523 & 0.0147 & 0.0428 & 0.0505 & 0.0196 & 0.0338 & 0.0311 & 0.0448 & 0.0432 & 0.0289 \\
\hline
\end{tabular}




\begin{tabular}{|c|c|c|c|c|c|c|c|c|c|c|c|}
\hline \multicolumn{12}{|c|}{ Magnafloc in unfiltered/undiluted Rideau Rive } \\
\hline \multicolumn{12}{|c|}{ In-line spectrophotometer (4mm path length) } \\
\hline $\begin{array}{l}\text { Wavelength } \\
(\mathrm{nm})\end{array}$ & $0 \mathrm{mg} / \mathrm{L}$ & $\begin{array}{c}0.1 \\
\mathrm{mg} / \mathrm{L}\end{array}$ & $\begin{array}{c}0.2 \\
\mathrm{mg} / \mathrm{L}\end{array}$ & $\begin{array}{c}0.3 \\
\mathrm{mg} / \mathrm{L}\end{array}$ & $\begin{array}{c}0.4 \\
\mathrm{mg} / \mathrm{L}\end{array}$ & $\begin{array}{c}0.5 \\
\mathrm{mg} / \mathrm{L}\end{array}$ & $\begin{array}{c}0.6 \\
\mathrm{mg} / \mathrm{L}\end{array}$ & $\begin{array}{c}0.7 \\
\mathrm{mg} / \mathrm{L}\end{array}$ & $\begin{array}{c}0.8 \\
\mathrm{mg} / \mathrm{L}\end{array}$ & $\begin{array}{c}0.9 \\
\mathrm{mg} / \mathrm{L}\end{array}$ & $\begin{array}{c}1.0 \\
\mathrm{mg} / \mathrm{L}\end{array}$ \\
\hline 189.8 & 0.8981 & 0.9123 & 0.9173 & 0.9219 & 0.9282 & 0.9320 & 0.9380 & 0.9430 & 0.9461 & 0.9512 & 0.9546 \\
\hline 192.8 & 0.7530 & 0.7614 & 0.7626 & 0.7648 & 0.7690 & 0.7716 & 0.7743 & 0.7763 & 0.7773 & 0.7814 & 0.7806 \\
\hline 195.8 & 0.6882 & 0.6960 & 0.6942 & 0.6961 & 0.6983 & 0.7028 & 0.7034 & 0.7051 & 0.7046 & 0.7073 & 0.7076 \\
\hline 198.9 & 0.6315 & 0.6372 & 0.6353 & 0.6370 & 0.6389 & 0.6413 & 0.6421 & 0.6436 & 0.6434 & 0.6445 & 0.6454 \\
\hline 201.9 & 0.5843 & 0.5888 & 0.5874 & 0.5883 & 0.5902 & 0.5922 & 0.5924 & 0.5935 & 0.5928 & 0.5951 & 0.5943 \\
\hline 204.9 & 0.5284 & 0.5338 & 0.5313 & 0.5323 & 0.5339 & 0.5377 & 0.5376 & 0.5385 & 0.5367 & 0.5391 & 0.5387 \\
\hline 208 & 0.4751 & 0.4798 & 0.4780 & 0.4795 & 0.4809 & 0.4831 & 0.4834 & 0.4845 & 0.4832 & 0.4845 & 0.4842 \\
\hline 211 & 0.4263 & 0.4305 & 0.4290 & 0.4298 & 0.4313 & 0.4337 & 0.4339 & 0.4352 & 0.4336 & 0.4354 & 0.4339 \\
\hline 214 & 0.3837 & 0.3884 & 0.3863 & 0.3874 & 0.3886 & 0.3916 & 0.3922 & 0.3928 & 0.3905 & 0.3924 & 0.3919 \\
\hline 217 & 0.3443 & 0.3485 & 0.3471 & 0.3481 & 0.3515 & 0.3513 & 0.3518 & 0.3531 & 0.3513 & 0.3521 & 0.3519 \\
\hline 220 & 0.3168 & 0.3210 & 0.3198 & 0.3200 & 0.3217 & 0.3240 & 0.3242 & 0.3255 & 0.3231 & 0.3248 & 0.3235 \\
\hline 223.1 & 0.2901 & 0.2941 & 0.2930 & 0.2938 & 0.2948 & 0.2975 & 0.2981 & 0.2983 & 0.2963 & 0.2973 & 0.2970 \\
\hline 226.1 & 0.2721 & 0.2756 & 0.2745 & 0.2758 & 0.2774 & 0.2789 & 0.2792 & 0.2804 & 0.2781 & 0.2790 & 0.2786 \\
\hline 229.1 & 0.2558 & 0.2609 & 0.2578 & 0.2584 & 0.2598 & 0.2623 & 0.2623 & 0.2637 & 0.2610 & 0.2625 & 0.2612 \\
\hline 232.1 & 0.2421 & 0.2459 & 0.2449 & 0.2456 & 0.2468 & 0.2491 & 0.2501 & 0.2507 & 0.2480 & 0.2490 & 0.2485 \\
\hline 235.1 & 0.2330 & 0.2368 & 0.2353 & 0.2363 & 0.2379 & 0.2392 & 0.2399 & 0.2407 & 0.2388 & 0.2392 & 0.2388 \\
\hline 238.1 & 0.2257 & 0.2286 & 0.2279 & 0.2280 & 0.2291 & 0.2317 & 0.2321 & 0.2332 & 0.2305 & 0.2320 & 0.2305 \\
\hline 241.1 & 0.2172 & 0.2207 & 0.2201 & 0.2218 & 0.2221 & 0.2242 & 0.2253 & 0.2260 & 0.2231 & 0.2235 & 0.2232 \\
\hline 244.1 & 0.2136 & 0.2174 & 0.2159 & 0.2172 & 0.2184 & 0.2201 & 0.2205 & 0.2220 & 0.2194 & 0.2198 & 0.2196 \\
\hline 247.1 & 0.2094 & 0.2113 & 0.2109 & 0.2111 & 0.2123 & 0.2153 & 0.2153 & 0.2166 & 0.2140 & 0.2154 & 0.2137 \\
\hline 250.1 & 0.2035 & 0.2069 & 0.2062 & 0.2069 & 0.2086 & 0.2122 & 0.2112 & 0.2115 & 0.2094 & 0.2095 & 0.2091 \\
\hline CONC & 0 & 0.1 & 0.2 & 0.3 & 0.4 & 0.5 & 0.6 & 0.7 & 0.8 & 0.9 & 1 \\
\hline $\begin{array}{l}\text { ABS at } \\
190 \mathrm{~nm}\end{array}$ & 0.8981 & 0.9123 & 0.9173 & 0.9219 & 0.9282 & 0.9320 & 0.9380 & 0.9430 & 0.9461 & 0.9512 & 0.9546 \\
\hline STDV & 0.0048 & 0.0023 & 0.0031 & 0.0016 & 0.0016 & 0.0013 & 0.0011 & 0.0015 & 0.0010 & 0.0010 & 0.0012 \\
\hline
\end{tabular}




\begin{tabular}{|c|c|c|c|c|c|c|c|c|c|c|c|}
\hline \multicolumn{12}{|c|}{ Magnafloc in unfiltered/diluted Rideau River water (3.2 NTU) (2018-03-21) } \\
\hline \multicolumn{12}{|c|}{ In-line spectrophotometer (4mm path length) } \\
\hline $\begin{array}{l}\text { Wavelength } \\
(\mathrm{nm})\end{array}$ & $\begin{array}{c}0 \\
\mathrm{mg} / \mathrm{L}\end{array}$ & $\begin{array}{c}0.1 \\
\mathrm{mg} / \mathrm{L}\end{array}$ & $\begin{array}{c}0.2 \\
\mathrm{mg} / \mathrm{L}\end{array}$ & $\begin{array}{c}0.3 \\
\mathrm{mg} / \mathrm{L}\end{array}$ & $\begin{array}{c}0.4 \\
\mathrm{mg} / \mathrm{L}\end{array}$ & $\begin{array}{c}0.5 \\
\mathrm{mg} / \mathrm{L}\end{array}$ & $\begin{array}{c}0.6 \\
\mathrm{mg} / \mathrm{L}\end{array}$ & $\begin{array}{c}0.7 \\
\mathrm{mg} / \mathrm{L}\end{array}$ & $\begin{array}{c}0.8 \\
\mathrm{mg} / \mathrm{L}\end{array}$ & $\begin{array}{c}0.9 \\
\mathrm{mg} / \mathrm{L}\end{array}$ & $\begin{array}{c}1.0 \\
\mathrm{mg} / \mathrm{L}\end{array}$ \\
\hline 189.8 & 0.4582 & 0.4663 & 0.4626 & 0.4626 & 0.4684 & 0.4710 & 0.4732 & 0.4748 & 0.4729 & 0.4719 & 0.4781 \\
\hline 192.8 & 0.3766 & 0.3830 & 0.3791 & 0.3785 & 0.3836 & 0.3855 & 0.3869 & 0.3870 & 0.3849 & 0.3865 & 0.3905 \\
\hline 195.8 & 0.3410 & 0.3480 & 0.3431 & 0.3425 & 0.3479 & 0.3509 & 0.3505 & 0.3503 & 0.3482 & 0.3504 & 0.3545 \\
\hline 198.9 & 0.3107 & 0.3174 & 0.3137 & 0.3122 & 0.3169 & 0.3178 & 0.3192 & 0.3192 & 0.3180 & 0.3179 & 0.3224 \\
\hline 201.9 & 0.2873 & 0.2934 & 0.2896 & 0.2882 & 0.2924 & 0.2937 & 0.2942 & 0.2939 & 0.2954 & 0.2936 & 0.2973 \\
\hline 204.9 & 0.2603 & 0.2665 & 0.2623 & 0.2611 & 0.2657 & 0.2662 & 0.2667 & 0.2661 & 0.2651 & 0.2675 & 0.2700 \\
\hline 208 & 0.2343 & 0.2398 & 0.2366 & 0.2354 & 0.2389 & 0.2395 & 0.2398 & 0.2397 & 0.2391 & 0.2398 & 0.2433 \\
\hline 211 & 0.2128 & 0.2157 & 0.2122 & 0.2106 & 0.2234 & 0.2153 & 0.2156 & 0.2147 & 0.2136 & 0.2147 & 0.2178 \\
\hline 214 & 0.1890 & 0.1947 & 0.1911 & 0.1897 & 0.1931 & 0.1939 & 0.1941 & 0.1935 & 0.1927 & 0.1950 & 0.1972 \\
\hline 217 & 0.1698 & 0.1750 & 0.1721 & 0.1703 & 0.1732 & 0.1739 & 0.1741 & 0.1738 & 0.1733 & 0.1741 & 0.1767 \\
\hline 220 & 0.1594 & 0.1613 & 0.1581 & 0.1565 & 0.1593 & 0.1603 & 0.1605 & 0.1597 & 0.1590 & 0.1593 & 0.1624 \\
\hline 223.1 & 0.1425 & 0.1478 & 0.1446 & 0.1428 & 0.1460 & 0.1465 & 0.1467 & 0.1457 & 0.1457 & 0.1463 & 0.1490 \\
\hline 226.1 & 0.1342 & 0.1388 & 0.1362 & 0.1344 & 0.1371 & 0.1378 & 0.1379 & 0.1371 & 0.1369 & 0.1376 & 0.1400 \\
\hline 229.1 & 0.1258 & 0.1306 & 0.1277 & 0.1260 & 0.1282 & 0.1294 & 0.1295 & 0.1287 & 0.1274 & 0.1278 & 0.1308 \\
\hline 232.1 & 0.1193 & 0.1242 & 0.1212 & 0.1193 & 0.1219 & 0.1225 & 0.1226 & 0.1217 & 0.1213 & 0.1225 & 0.1247 \\
\hline 235.1 & 0.1151 & 0.1193 & 0.1168 & 0.1152 & 0.1173 & 0.1178 & 0.1182 & 0.1169 & 0.1169 & 0.1178 & 0.1200 \\
\hline 238.1 & 0.1111 & 0.1157 & 0.1133 & 0.1112 & 0.1136 & 0.1143 & 0.1143 & 0.1133 & 0.1128 & 0.1136 & 0.1162 \\
\hline 241.1 & 0.1067 & 0.1114 & 0.1089 & 0.1064 & 0.1090 & 0.1095 & 0.1097 & 0.1085 & 0.1084 & 0.1092 & 0.1120 \\
\hline 244.1 & 0.1057 & 0.1097 & 0.1075 & 0.1057 & 0.1078 & 0.1084 & 0.1084 & 0.1074 & 0.1074 & 0.1086 & 0.1103 \\
\hline 247.1 & 0.1031 & 0.1075 & 0.1050 & 0.1030 & 0.1047 & 0.1060 & 0.1058 & 0.1048 & 0.1040 & 0.1053 & 0.1073 \\
\hline 250.1 & 0.1001 & 0.1045 & 0.1021 & 0.0996 & 0.1021 & 0.1025 & 0.1030 & 0.1013 & 0.1018 & 0.1018 & 0.1049 \\
\hline $\mathrm{CONC}$ & 0 & 0.1 & 0.2 & 0.3 & 0.4 & 0.5 & 0.6 & 0.7 & 0.8 & 0.9 & 1 \\
\hline $\begin{array}{l}\text { ABS at } \\
190 \mathrm{NM}\end{array}$ & 0.4582 & 0.4663 & 0.4626 & 0.4626 & 0.4684 & 0.4710 & 0.4732 & 0.4748 & 0.4729 & 0.4719 & 0.4781 \\
\hline STDV & 0.0007 & 0.0014 & 0.0005 & 0.0010 & 0.0017 & 0.0008 & 0.0010 & 0.0023 & 0.0002 & 0.0003 & 0.0023 \\
\hline
\end{tabular}




\begin{tabular}{|c|c|c|c|c|c|c|c|c|c|c|c|}
\hline \multicolumn{12}{|c|}{ Magnafloc in unfiltered/diluted Rideau River water (3.2 NTU) (2018-03-21) } \\
\hline \multicolumn{12}{|c|}{ Desktop spectrophotometer (1 $\mathrm{cm}$ path length) } \\
\hline $\begin{array}{c}\text { Wavelength } \\
(\mathrm{nm})\end{array}$ & $0 \mathrm{mg} / \mathrm{L}$ & $\begin{array}{c}0.1 \\
\mathrm{mg} / \mathrm{L} \\
\end{array}$ & $\begin{array}{c}0.2 \\
\mathrm{mg} / \mathrm{L}\end{array}$ & $\begin{array}{c}0.3 \\
\mathrm{mg} / \mathrm{L}\end{array}$ & $\begin{array}{c}0.4 \\
\mathrm{mg} / \mathrm{L} \\
\end{array}$ & $\begin{array}{c}0.5 \\
\mathrm{mg} / \mathrm{L}\end{array}$ & $\begin{array}{c}0.6 \\
\mathrm{mg} / \mathrm{L} \\
\end{array}$ & $\begin{array}{c}0.7 \\
\mathrm{mg} / \mathrm{L}\end{array}$ & $\begin{array}{c}0.8 \\
\mathrm{mg} / \mathrm{L}\end{array}$ & $\begin{array}{c}0.9 \\
\mathrm{mg} / \mathrm{L}\end{array}$ & $\begin{array}{c}1.0 \\
\mathrm{mg} / \mathrm{L}\end{array}$ \\
\hline 250 & 0.1595 & 0.1608 & 0.1600 & 0.1602 & 0.1630 & 0.1610 & 0.1621 & 0.1677 & 0.1669 & 0.1641 & 0.1652 \\
\hline 249 & 0.1644 & 0.1655 & 0.1648 & 0.1651 & 0.1678 & 0.1658 & 0.1669 & 0.1724 & 0.1718 & 0.1689 & 0.1699 \\
\hline 248 & 0.1692 & 0.1704 & 0.1697 & 0.1699 & 0.1727 & 0.1706 & 0.1718 & 0.1774 & 0.1767 & 0.1738 & 0.1749 \\
\hline 247 & 0.1742 & 0.1753 & 0.1747 & 0.1748 & 0.1775 & 0.1755 & 0.1766 & 0.1823 & 0.1816 & 0.1787 & 0.1797 \\
\hline 246 & 0.1787 & 0.1799 & 0.1794 & 0.1794 & 0.1822 & 0.1801 & 0.1814 & 0.1871 & 0.1863 & 0.1834 & 0.1844 \\
\hline 245 & 0.1832 & 0.1845 & 0.1839 & 0.1839 & 0.1865 & 0.1846 & 0.1858 & 0.1917 & 0.1909 & 0.1881 & 0.1889 \\
\hline 244 & 0.1874 & 0.1886 & 0.1879 & 0.1880 & 0.1907 & 0.1888 & 0.1899 & 0.1958 & 0.1950 & 0.1921 & 0.1929 \\
\hline 243 & 0.1912 & 0.1923 & 0.1917 & 0.1919 & 0.1944 & 0.1925 & 0.1937 & 0.1994 & 0.1987 & 0.1960 & 0.1969 \\
\hline 242 & 0.1944 & 0.1957 & 0.1950 & 0.1953 & 0.1978 & 0.1959 & 0.1970 & 0.2030 & 0.2022 & 0.1992 & 0.2002 \\
\hline 241 & 0.1973 & 0.1986 & 0.1979 & 0.1981 & 0.2007 & 0.1987 & 0.1999 & 0.2059 & 0.2052 & 0.2022 & 0.2031 \\
\hline 240 & 0.1998 & 0.2011 & 0.2004 & 0.2006 & 0.2032 & 0.2013 & 0.2025 & 0.2084 & 0.2077 & 0.2048 & 0.2058 \\
\hline 239 & 0.2020 & 0.2032 & 0.2025 & 0.2025 & 0.2054 & 0.2033 & 0.2047 & 0.2106 & 0.2099 & 0.2070 & 0.2080 \\
\hline 238 & 0.2037 & 0.2050 & 0.2042 & 0.2045 & 0.2072 & 0.2052 & 0.2065 & 0.2125 & 0.2118 & 0.2087 & 0.2099 \\
\hline 237 & 0.2054 & 0.2066 & 0.2058 & 0.2061 & 0.2088 & 0.2069 & 0.2080 & 0.2141 & 0.2134 & 0.2103 & 0.2115 \\
\hline 236 & 0.2067 & 0.2080 & 0.2072 & 0.2074 & 0.2102 & 0.2083 & 0.2095 & 0.2155 & 0.2150 & 0.2118 & 0.2128 \\
\hline 235 & 0.2080 & 0.2092 & 0.2085 & 0.2086 & 0.2116 & 0.2095 & 0.2107 & 0.2171 & 0.2162 & 0.2129 & 0.2144 \\
\hline 234 & 0.2093 & 0.2106 & 0.2097 & 0.2100 & 0.2131 & 0.2108 & 0.2121 & 0.2183 & 0.2175 & 0.2145 & 0.2156 \\
\hline 233 & 0.2107 & 0.2122 & 0.2111 & 0.2114 & 0.2146 & 0.2122 & 0.2136 & 0.2199 & 0.2191 & 0.2160 & 0.2174 \\
\hline 232 & 0.2124 & 0.2139 & 0.2129 & 0.2131 & 0.2163 & 0.2140 & 0.2154 & 0.2216 & 0.2209 & 0.2177 & 0.2190 \\
\hline 231 & 0.2144 & 0.2158 & 0.2148 & 0.2151 & 0.2185 & 0.2160 & 0.2173 & 0.2238 & 0.2230 & 0.2198 & 0.2212 \\
\hline 230 & 0.2166 & 0.2181 & 0.2171 & 0.2173 & 0.2210 & 0.2182 & 0.2198 & 0.2262 & 0.2253 & 0.2221 & 0.2235 \\
\hline 229 & 0.2193 & 0.2209 & 0.2198 & 0.2201 & 0.2239 & 0.2211 & 0.2225 & 0.2290 & 0.2281 & 0.2249 & 0.2263 \\
\hline 228 & 0.2227 & 0.2241 & 0.2231 & 0.2232 & 0.2275 & 0.2243 & 0.2258 & 0.2323 & 0.2315 & 0.2281 & 0.2296 \\
\hline 227 & 0.2265 & 0.2279 & 0.2269 & 0.2271 & 0.2316 & 0.2282 & 0.2297 & 0.2363 & 0.2353 & 0.2321 & 0.2334 \\
\hline 226 & 0.2311 & 0.2325 & 0.2313 & 0.2315 & 0.2362 & 0.2326 & 0.2343 & 0.2408 & 0.2400 & 0.2365 & 0.2379 \\
\hline 225 & 0.2362 & 0.2376 & 0.2364 & 0.2366 & 0.2416 & 0.2379 & 0.2395 & 0.2461 & 0.2453 & 0.2418 & 0.2432 \\
\hline 224 & 0.2422 & 0.2438 & 0.2424 & 0.2427 & 0.2478 & 0.2439 & 0.2455 & 0.2523 & 0.2515 & 0.2479 & 0.2493 \\
\hline 223 & 0.2490 & 0.2506 & 0.2492 & 0.2495 & 0.2550 & 0.2508 & 0.2526 & 0.2593 & 0.2583 & 0.2548 & 0.2564 \\
\hline 222 & 0.2569 & 0.2585 & 0.2570 & 0.2573 & 0.2631 & 0.2587 & 0.2604 & 0.2672 & 0.2663 & 0.2629 & 0.2642 \\
\hline 221 & 0.2656 & 0.2672 & 0.2657 & 0.2661 & 0.2720 & 0.2675 & 0.2693 & 0.2761 & 0.2749 & 0.2716 & 0.2730 \\
\hline 220 & 0.2754 & 0.2771 & 0.2755 & 0.2757 & 0.2821 & 0.2774 & 0.2790 & 0.2860 & 0.2849 & 0.2815 & 0.2829 \\
\hline 219 & 0.2861 & 0.2878 & 0.2861 & 0.2865 & 0.2930 & 0.2881 & 0.2899 & 0.2969 & 0.2958 & 0.2923 & 0.2940 \\
\hline 218 & 0.2979 & 0.2996 & 0.2980 & 0.2983 & 0.3052 & 0.2999 & 0.3018 & 0.3090 & 0.3078 & 0.3042 & 0.3058 \\
\hline 217 & 0.3105 & 0.3123 & 0.3105 & 0.3108 & 0.3180 & 0.3126 & 0.3143 & 0.3218 & 0.3203 & 0.3169 & 0.3184 \\
\hline 216 & 0.3240 & 0.3257 & 0.3239 & 0.3242 & 0.3316 & 0.3261 & 0.3280 & 0.3354 & 0.3339 & 0.3305 & 0.3322 \\
\hline 215 & 0.3380 & 0.3398 & 0.3379 & 0.3383 & 0.3460 & 0.3401 & 0.3420 & 0.3497 & 0.3481 & 0.3446 & 0.3462 \\
\hline
\end{tabular}




\begin{tabular}{|c|c|c|c|c|c|c|c|c|c|c|c|}
\hline 214 & 0.3529 & 0.3547 & 0.3527 & 0.3531 & 0.3611 & 0.3549 & 0.3569 & 0.3647 & 0.3631 & 0.3595 & 0.3613 \\
\hline 213 & 0.3681 & 0.3698 & 0.3677 & 0.3681 & 0.3767 & 0.3702 & 0.3722 & 0.3801 & 0.3785 & 0.3747 & 0.3768 \\
\hline 212 & 0.3839 & 0.3856 & 0.3834 & 0.3838 & 0.3926 & 0.3861 & 0.3882 & 0.3961 & 0.3944 & 0.3907 & 0.3928 \\
\hline 211 & 0.3997 & 0.4016 & 0.3994 & 0.3997 & 0.4089 & 0.4020 & 0.4043 & 0.4124 & 0.4105 & 0.4069 & 0.4087 \\
\hline 210 & 0.4162 & 0.4179 & 0.4156 & 0.4159 & 0.4254 & 0.4183 & 0.4206 & 0.4290 & 0.4271 & 0.4233 & 0.4252 \\
\hline 209 & 0.4323 & 0.4341 & 0.4318 & 0.4320 & 0.4421 & 0.4348 & 0.4370 & 0.4454 & 0.4437 & 0.4396 & 0.4416 \\
\hline 208 & 0.4488 & 0.4506 & 0.4481 & 0.4485 & 0.4588 & 0.4511 & 0.4536 & 0.4621 & 0.4603 & 0.4559 & 0.4582 \\
\hline 207 & 0.4649 & 0.4668 & 0.4643 & 0.4646 & 0.4752 & 0.4676 & 0.4700 & 0.4787 & 0.4769 & 0.4726 & 0.4746 \\
\hline 206 & 0.4814 & 0.4832 & 0.4804 & 0.4808 & 0.4920 & 0.4839 & 0.4864 & 0.4957 & 0.4935 & 0.4889 & 0.4912 \\
\hline 205 & 0.4976 & 0.4999 & 0.4971 & 0.4974 & 0.5090 & 0.5006 & 0.5032 & 0.5127 & 0.5103 & 0.5058 & 0.5079 \\
\hline 20 & 0.5149 & 0.5168 & 0.5140 & 0.5142 & 0.5264 & 0.5179 & 0.5203 & 0.5302 & 0.5278 & 0.5232 & 0.5251 \\
\hline 203 & 0.5324 & 0.5343 & 0.5313 & 0.5316 & 0.5442 & 0.5355 & 0.5381 & 0.5481 & 0.5458 & 0.5407 & 0.5430 \\
\hline 202 & 0.5508 & 0.5527 & 0.5496 & 0.5499 & 0.5630 & 0.5540 & 0.5570 & 0.5672 & 0.5645 & 0.5595 & 0.5618 \\
\hline 201 & 0.5705 & 0.5725 & 0.5693 & 0.5695 & 0.5834 & 0.5741 & 0.5772 & 0.5879 & 0.5850 & 0.5798 & 0.5821 \\
\hline 200 & 0.5925 & 0.5944 & 0.5914 & 0.5916 & 0.6060 & 0.5967 & 0.5998 & 0.6107 & 0.6078 & 0.6024 & 0.6047 \\
\hline 199 & 0.6174 & 0.6196 & 0.6163 & 0.6164 & 0.6318 & 0.6221 & 0.6256 & 0.6367 & 0.6334 & 0.6281 & 0.6308 \\
\hline 198 & 0.6460 & 0.6481 & 0.6446 & 0.6448 & 0.6612 & 0.6511 & 0.6548 & 0.6664 & 0.6627 & 0.6573 & 0.6602 \\
\hline 197 & 0.6801 & 0.6825 & 0.6789 & 0.6788 & 0.6966 & 0.6861 & 0.6913 & 0.7019 & 0.6979 & 0.6926 & 0.6969 \\
\hline 196 & 0.7202 & 0.7227 & 0.7192 & 0.7189 & 0.7379 & 0.7272 & 0.7311 & 0.7434 & 0.7389 & 0.7336 & 0.7371 \\
\hline 195 & 0.7717 & 0.7748 & 0.7709 & 0.7707 & 0.7907 & 0.7798 & 0.7825 & 0.7964 & 0.7916 & 0.7864 & 0.7887 \\
\hline 194 & 0.8304 & 0.8336 & 0.8298 & 0.8294 & 0.8509 & 0.8399 & 0.8441 & 0.8569 & 0.8515 & 0.8465 & 0.8509 \\
\hline 193 & 0.9038 & 0.9074 & 0.9035 & 0.9031 & 0.9256 & 0.9148 & 0.9189 & 0.9319 & 0.9259 & 0.9211 & 0.9262 \\
\hline 192 & 1.0011 & 1.0052 & 1.0011 & 1.0000 & 1.0243 & 1.0133 & 1.0174 & 1.0310 & 1.0240 & 1.0197 & 1.0250 \\
\hline 191 & 1.1289 & 1.1336 & 1.1292 & 1.1276 & 1.1542 & 1.1428 & 1.1496 & 1.1612 & 1.1532 & 1.1492 & 1.1580 \\
\hline 190 & 1.2809 & 1.2862 & 1.2815 & 1.2801 & 1.3076 & 1.2972 & 1.2966 & 1.3147 & 1.3052 & 1.3025 & 1.3059 \\
\hline CONC & 0 & 0.1 & 0.2 & 0.3 & 0.4 & 0.5 & 0.6 & 0.7 & 0.8 & 0.9 & 1 \\
\hline $\begin{array}{l}\text { ABS AT } \\
190 \mathrm{~nm}\end{array}$ & 1.2809 & 1.2862 & 1.2815 & 1.2801 & 1.3076 & 1.2972 & 1.2966 & 1.3147 & 1.3052 & 1.3025 & 1.3059 \\
\hline STDV & 0.0023 & 0.0067 & 0.0048 & 0.0034 & 0.0062 & 0.0015 & 0.0052 & 0.0063 & 0.0010 & 0.0039 & 0.0056 \\
\hline
\end{tabular}




\begin{tabular}{|c|c|c|c|c|c|c|c|c|c|c|c|}
\hline \multicolumn{12}{|c|}{ Magnafloc in filtered/undiluted Rideau River Water (3.2 NTU) (2018-03-19) } \\
\hline \multicolumn{12}{|c|}{ Desktop spectrophotometer (1 $\mathrm{cm}$ path length) } \\
\hline $\begin{array}{l}\text { Wavelength } \\
\quad(\mathrm{nm})\end{array}$ & $0 \mathrm{mg} / \mathrm{L}$ & $\begin{array}{c}0.1 \\
\mathrm{mg} / \mathrm{L}\end{array}$ & $\begin{array}{c}0.2 \\
\mathrm{mg} / \mathrm{L}\end{array}$ & $\begin{array}{c}0.3 \\
\mathrm{mg} / \mathrm{L}\end{array}$ & $\begin{array}{c}0.4 \\
\mathrm{mg} / \mathrm{L}\end{array}$ & $\begin{array}{c}0.5 \\
\mathrm{mg} / \mathrm{L}\end{array}$ & $\begin{array}{c}0.6 \\
\mathrm{mg} / \mathrm{L}\end{array}$ & $\begin{array}{c}0.7 \\
\mathrm{mg} / \mathrm{L}\end{array}$ & $\begin{array}{c}0.8 \\
\mathrm{mg} / \mathrm{L}\end{array}$ & $\begin{array}{c}0.9 \\
\mathrm{mg} / \mathrm{L}\end{array}$ & $\begin{array}{c}1.0 \\
\mathrm{mg} / \mathrm{L}\end{array}$ \\
\hline 250 & 0.2542 & 0.2542 & 0.2538 & 0.2533 & 0.2538 & 0.2531 & 0.2539 & 0.2539 & 0.2538 & 0.2541 & 0.2535 \\
\hline 249 & 0.2599 & 0.2598 & 0.2594 & 0.2590 & 0.2594 & 0.2588 & 0.2595 & 0.2595 & 0.2595 & 0.2596 & 0.2590 \\
\hline 248 & 0.2657 & 0.2657 & 0.2653 & 0.2648 & 0.2651 & 0.2646 & 0.2653 & 0.2652 & 0.2652 & 0.2652 & 0.2648 \\
\hline 247 & 0.2713 & 0.2713 & 0.2709 & 0.2705 & 0.2708 & 0.2702 & 0.2711 & 0.2709 & 0.2710 & 0.2711 & 0.2705 \\
\hline 246 & 0.2770 & 0.2770 & 0.2766 & 0.2760 & 0.2764 & 0.2758 & 0.2766 & 0.2766 & 0.2765 & 0.2767 & 0.2761 \\
\hline 245 & 0.2826 & 0.2824 & 0.2820 & 0.2816 & 0.2819 & 0.2814 & 0.2821 & 0.2820 & 0.2821 & 0.2821 & 0.2817 \\
\hline 244 & 0.2878 & 0.2877 & 0.2873 & 0.2869 & 0.2872 & 0.2866 & 0.2874 & 0.2872 & 0.2873 & 0.2873 & 0.2869 \\
\hline 243 & 0.2927 & 0.2926 & 0.2922 & 0.2918 & 0.2921 & 0.2915 & 0.2924 & 0.2923 & 0.2922 & 0.2924 & 0.2919 \\
\hline 242 & 0.2974 & 0.2972 & 0.2969 & 0.2965 & 0.2969 & 0.2962 & 0.2969 & 0.2969 & 0.2968 & 0.2969 & 0.2965 \\
\hline 241 & 0.3017 & 0.3016 & 0.3011 & 0.3007 & 0.3010 & 0.3005 & 0.3012 & 0.3012 & 0.3011 & 0.3012 & 0.3008 \\
\hline 240 & 0.3057 & 0.3055 & 0.3051 & 0.3047 & 0.3050 & 0.3045 & 0.3053 & 0.3051 & 0.3051 & 0.3052 & 0.3048 \\
\hline 239 & 0.3095 & 0.3093 & 0.3089 & 0.3085 & 0.3087 & 0.3083 & 0.3090 & 0.3088 & 0.3088 & 0.3089 & 0.3085 \\
\hline 238 & 0.3129 & 0.3128 & 0.3125 & 0.3120 & 0.3123 & 0.3117 & 0.3125 & 0.3124 & 0.3124 & 0.3125 & 0.3120 \\
\hline 237 & 0.3164 & 0.3163 & 0.3160 & 0.3155 & 0.3157 & 0.3152 & 0.3160 & 0.3160 & 0.3158 & 0.3160 & 0.3156 \\
\hline 236 & 0.3199 & 0.3199 & 0.3195 & 0.3191 & 0.3193 & 0.3189 & 0.3196 & 0.3196 & 0.3194 & 0.3195 & 0.3190 \\
\hline 235 & 0.3237 & 0.3236 & 0.3232 & 0.3227 & 0.3230 & 0.3224 & 0.3232 & 0.3232 & 0.3230 & 0.3232 & 0.3227 \\
\hline 234 & 0.3278 & 0.3276 & 0.3273 & 0.3267 & 0.3268 & 0.3263 & 0.3271 & 0.3271 & 0.3269 & 0.3271 & 0.3266 \\
\hline 233 & 0.3321 & 0.3320 & 0.3315 & 0.3311 & 0.3312 & 0.3308 & 0.3315 & 0.3315 & 0.3313 & 0.3315 & 0.3309 \\
\hline 232 & 0.3370 & 0.3369 & 0.3365 & 0.3358 & 0.3360 & 0.3355 & 0.3361 & 0.3362 & 0.3360 & 0.3363 & 0.3358 \\
\hline 231 & 0.3424 & 0.3423 & 0.3418 & 0.3412 & 0.3413 & 0.3408 & 0.3416 & 0.3416 & 0.3414 & 0.3416 & 0.3410 \\
\hline 230 & 0.3485 & 0.3483 & 0.3478 & 0.3473 & 0.3473 & 0.3468 & 0.3475 & 0.3475 & 0.3474 & 0.3475 & 0.3470 \\
\hline 229 & 0.3551 & 0.3551 & 0.3545 & 0.3540 & 0.3540 & 0.3535 & 0.3541 & 0.3541 & 0.3538 & 0.3540 & 0.3536 \\
\hline 228 & 0.3626 & 0.3626 & 0.3620 & 0.3615 & 0.3614 & 0.3610 & 0.3616 & 0.3616 & 0.3613 & 0.3616 & 0.3610 \\
\hline 227 & 0.3711 & 0.3711 & 0.3705 & 0.3699 & 0.3698 & 0.3692 & 0.3699 & 0.3699 & 0.3697 & 0.3699 & 0.3694 \\
\hline 226 & 0.3804 & 0.3805 & 0.3799 & 0.3794 & 0.3792 & 0.3787 & 0.3792 & 0.3792 & 0.3789 & 0.3793 & 0.3786 \\
\hline 225 & 0.3907 & 0.3910 & 0.3904 & 0.3898 & 0.3895 & 0.3889 & 0.3896 & 0.3895 & 0.3891 & 0.3895 & 0.3889 \\
\hline 224 & 0.4022 & 0.4026 & 0.4020 & 0.4015 & 0.4011 & 0.4005 & 0.4011 & 0.4009 & 0.4006 & 0.4010 & 0.4003 \\
\hline 223 & 0.4148 & 0.4154 & 0.4149 & 0.4142 & 0.4138 & 0.4132 & 0.4139 & 0.4136 & 0.4132 & 0.4136 & 0.4130 \\
\hline 222 & 0.4288 & 0.4296 & 0.4290 & 0.4285 & 0.4279 & 0.4273 & 0.4279 & 0.4277 & 0.4273 & 0.4276 & 0.4269 \\
\hline 221 & 0.4439 & 0.4450 & 0.4445 & 0.4437 & 0.4431 & 0.4425 & 0.4431 & 0.4429 & 0.4424 & 0.4427 & 0.4421 \\
\hline 220 & 0.4604 & 0.4619 & 0.4613 & 0.4608 & 0.4599 & 0.4593 & 0.4598 & 0.4597 & 0.4591 & 0.4594 & 0.4586 \\
\hline 219 & 0.4779 & 0.4799 & 0.4794 & 0.4786 & 0.4778 & 0.4772 & 0.4776 & 0.4775 & 0.4767 & 0.4772 & 0.4765 \\
\hline 218 & 0.4971 & 0.4994 & 0.4988 & 0.4982 & 0.4972 & 0.4965 & 0.4969 & 0.4968 & 0.4961 & 0.4964 & 0.4957 \\
\hline 217 & 0.5170 & 0.5197 & 0.5193 & 0.5185 & 0.5173 & 0.5167 & 0.5170 & 0.5169 & 0.5163 & 0.5165 & 0.5156 \\
\hline 216 & 0.5380 & 0.5411 & 0.5406 & 0.5399 & 0.5386 & 0.5380 & 0.5383 & 0.5384 & 0.5374 & 0.5378 & 0.5369 \\
\hline
\end{tabular}




\begin{tabular}{|c|c|c|c|c|c|c|c|c|c|c|c|}
\hline 215 & 0.5596 & 0.5631 & 0.5626 & 0.5619 & 0.5604 & 0.5599 & 0.5602 & 0.5602 & 0.5592 & 0.5597 & 0.5586 \\
\hline 214 & 0.5821 & 0.5862 & 0.5856 & 0.5848 & 0.5832 & 0.5827 & 0.5829 & 0.5830 & 0.5820 & 0.5824 & 0.5812 \\
\hline 213 & 0.6049 & 0.6092 & 0.6086 & 0.6079 & 0.6061 & 0.6056 & 0.6058 & 0.6060 & 0.6048 & 0.6052 & 0.6041 \\
\hline 212 & 0.6281 & 0.6329 & 0.6322 & 0.6315 & 0.6297 & 0.6292 & 0.6294 & 0.6295 & 0.6284 & 0.6287 & 0.6276 \\
\hline 211 & 0.6513 & 0.6564 & 0.6557 & 0.6551 & 0.6533 & 0.6526 & 0.6527 & 0.6530 & 0.6517 & 0.6522 & 0.6508 \\
\hline 210 & 0.6748 & 0.6802 & 0.6795 & 0.6790 & 0.6768 & 0.6763 & 0.6764 & 0.6767 & 0.6752 & 0.6757 & 0.6743 \\
\hline 209 & 0.6979 & 0.7036 & 0.7030 & 0.7024 & 0.7002 & 0.6996 & 0.6998 & 0.7002 & 0.6986 & 0.6991 & 0.6977 \\
\hline 208 & 0.7210 & 0.7269 & 0.7263 & 0.7258 & 0.7235 & 0.7229 & 0.7231 & 0.7234 & 0.7219 & 0.7223 & 0.7209 \\
\hline 207 & 0.7439 & 0.7500 & 0.7494 & 0.7487 & 0.7463 & 0.7457 & 0.7460 & 0.7464 & 0.7449 & 0.7453 & 0.7440 \\
\hline 206 & 0.7665 & 0.7730 & 0.7723 & 0.7716 & 0.7695 & 0.7687 & 0.7692 & 0.7694 & 0.7679 & 0.7683 & 0.7669 \\
\hline 205 & 0.7895 & 0.7960 & 0.7953 & 0.7948 & 0.7922 & 0.7917 & 0.7922 & 0.7925 & 0.7909 & 0.7915 & 0.7900 \\
\hline 204 & 0.8137 & 0.8201 & 0.8194 & 0.8190 & 0.8165 & 0.8159 & 0.8164 & 0.8168 & 0.8151 & 0.8158 & 0.8143 \\
\hline 203 & 0.8386 & 0.8450 & 0.8444 & 0.8438 & 0.8413 & 0.8408 & 0.8414 & 0.8420 & 0.8403 & 0.8411 & 0.8396 \\
\hline 202 & 0.8657 & 0.8720 & 0.8716 & 0.8709 & 0.8685 & 0.8681 & 0.8689 & 0.8694 & 0.8677 & 0.8687 & 0.8671 \\
\hline 201 & 0.8961 & 0.9019 & 0.9016 & 0.9009 & 0.8985 & 0.8980 & 0.8990 & 0.8995 & 0.8980 & 0.8993 & 0.8978 \\
\hline 200 & 0.9311 & 0.9363 & 0.9361 & 0.9352 & 0.9330 & 0.9328 & 0.9341 & 0.9343 & 0.9329 & 0.9345 & 0.9331 \\
\hline 199 & 0.9714 & 0.9758 & 0.9759 & 0.9747 & 0.9731 & 0.9728 & 0.9739 & 0.9747 & 0.9735 & 0.9753 & 0.9737 \\
\hline 198 & 1.0183 & 1.0218 & 1.0219 & 1.0212 & 1.0192 & 1.0195 & 1.0211 & 1.0218 & 1.0202 & 1.0228 & 1.0216 \\
\hline 197 & 1.0733 & 1.0764 & 1.0768 & 1.0758 & 1.0744 & 1.0748 & 1.0765 & 1.0773 & 1.0762 & 1.0791 & 1.0777 \\
\hline 196 & 1.1381 & 1.1409 & 1.1414 & 1.1403 & 1.1392 & 1.1398 & 1.1417 & 1.1426 & 1.1418 & 1.1454 & 1.1441 \\
\hline 195 & 1.2192 & 1.2212 & 1.2222 & 1.2214 & 1.2200 & 1.2210 & 1.2233 & 1.2246 & 1.2220 & 1.2279 & 1.2268 \\
\hline 194 & 1.3127 & 1.3143 & 1.3157 & 1.3146 & 1.3135 & 1.3152 & 1.3175 & 1.3190 & 1.3183 & 1.3229 & 1.3217 \\
\hline 193 & 1.4263 & 1.4275 & 1.4291 & 1.4280 & 1.4269 & 1.4288 & 1.4317 & 1.4334 & 1.4326 & 1.4384 & 1.4366 \\
\hline 192 & 1.5714 & 1.5711 & 1.5730 & 1.5719 & 1.5708 & 1.5730 & 1.5763 & 1.5777 & 1.5802 & 1.5841 & 1.5819 \\
\hline 191 & 1.7568 & 1.7568 & 1.7585 & 1.7565 & 1.7554 & 1.7580 & 1.7609 & 1.7630 & 1.7622 & 1.7697 & 1.7671 \\
\hline 190 & 1.9616 & 1.9601 & 1.9631 & 1.9613 & 1.9597 & 1.9623 & 1.9655 & 1.9678 & 1.9627 & 1.9748 & 1.9724 \\
\hline $\mathrm{CONC}$ & 0 & 0.1 & 0.2 & 0.3 & 0.4 & 0.5 & 0.6 & 0.7 & 0.8 & 0.9 & 1 \\
\hline $\begin{array}{l}\text { ABS at } \\
190 \mathrm{NM}\end{array}$ & 1.9616 & 1.9601 & 1.9631 & 1.9613 & 1.9597 & 1.9623 & 1.9655 & 1.9678 & 1.9627 & 1.9748 & 1.9724 \\
\hline STDV & 0.0018 & 0.0027 & 0.0035 & 0.0038 & 0.0018 & 0.0075 & 0.0039 & 0.0049 & 0.0003 & 0.0117 & 0.0007 \\
\hline
\end{tabular}




\begin{tabular}{|c|c|c|c|c|c|c|c|c|c|c|c|}
\hline \multicolumn{12}{|c|}{ Magnafloc in filtered/undiluted Rideau River Water (3.2 NTU) (2018-03-19) } \\
\hline \multicolumn{12}{|c|}{ In-line spectrophotometer ( $4 \mathrm{~mm}$ path length) } \\
\hline $\begin{array}{l}\text { Wavelength } \\
(\mathrm{nm})\end{array}$ & $\begin{array}{c}0 \\
\mathrm{mg} / \mathrm{L}\end{array}$ & $\begin{array}{c}0.1 \\
\mathrm{mg} / \mathrm{L}\end{array}$ & $\begin{array}{c}0.2 \\
\mathrm{mg} / \mathrm{L}\end{array}$ & $\begin{array}{c}0.3 \\
\mathrm{mg} / \mathrm{L}\end{array}$ & $\begin{array}{c}0.4 \\
\mathrm{mg} / \mathrm{L}\end{array}$ & $\begin{array}{c}0.5 \\
\mathrm{mg} / \mathrm{L}\end{array}$ & $\begin{array}{c}0.6 \\
\mathrm{mg} / \mathrm{L}\end{array}$ & $\begin{array}{c}0.7 \\
\mathrm{mg} / \mathrm{L}\end{array}$ & $\begin{array}{c}0.8 \\
\mathrm{mg} / \mathrm{L}\end{array}$ & $\begin{array}{c}0.9 \\
\mathrm{mg} / \mathrm{L}\end{array}$ & $\begin{array}{c}1.0 \\
\mathrm{mg} / \mathrm{L}\end{array}$ \\
\hline 189.8 & 1.0326 & 1.0451 & 1.0460 & 1.0491 & 1.0480 & 1.0534 & 1.0519 & 1.0532 & 1.0544 & 1.0576 & 1.0636 \\
\hline 192.8 & 0.8172 & 0.8291 & 0.8292 & 0.8302 & 0.8298 & 0.8310 & 0.8315 & 0.8325 & 0.8303 & 0.8330 & 0.8362 \\
\hline 195.8 & 0.7201 & 0.7332 & 0.7306 & 0.7319 & 0.7311 & 0.7311 & 0.7298 & 0.7322 & 0.7301 & 0.7316 & 0.7324 \\
\hline 198.9 & 0.6571 & 0.6701 & 0.6665 & 0.6675 & 0.6663 & 0.6661 & 0.6637 & 0.6658 & 0.6655 & 0.6654 & 0.6657 \\
\hline 201.9 & 0.6031 & 0.6137 & 0.6115 & 0.6122 & 0.6101 & 0.6118 & 0.6088 & 0.6104 & 0.6098 & 0.6100 & 0.6105 \\
\hline 204.9 & 0.5501 & 0.5587 & 0.5575 & 0.5578 & 0.5560 & 0.5571 & 0.5556 & 0.5557 & 0.5556 & 0.5557 & 0.5561 \\
\hline 208 & 0.4989 & 0.5070 & 0.5052 & 0.5057 & 0.5043 & 0.5047 & 0.5042 & 0.5048 & 0.5036 & 0.5043 & 0.5042 \\
\hline 211 & 0.4511 & 0.4588 & 0.4563 & 0.4562 & 0.4557 & 0.4556 & 0.4544 & 0.4559 & 0.4548 & 0.4554 & 0.4556 \\
\hline 214 & 0.4045 & 0.4106 & 0.4082 & 0.4085 & 0.4075 & 0.4083 & 0.4067 & 0.4081 & 0.4074 & 0.4082 & 0.4080 \\
\hline 217 & 0.3599 & 0.3641 & 0.3623 & 0.3630 & 0.3618 & 0.3633 & 0.3618 & 0.3628 & 0.3627 & 0.3630 & 0.3630 \\
\hline 220 & 0.3257 & 0.3288 & 0.3279 & 0.3280 & 0.3278 & 0.3283 & 0.3284 & 0.3284 & 0.3281 & 0.3287 & 0.3294 \\
\hline 223.1 & 0.2981 & 0.3017 & 0.3001 & 0.3000 & 0.3005 & 0.3001 & 0.3001 & 0.3008 & 0.3002 & 0.3012 & 0.3014 \\
\hline 226.1 & 0.2749 & 0.2781 & 0.2759 & 0.2762 & 0.2764 & 0.2766 & 0.2758 & 0.2769 & 0.2766 & 0.2776 & 0.2775 \\
\hline 229.1 & 0.2567 & 0.2592 & 0.2574 & 0.2578 & 0.2580 & 0.2586 & 0.2575 & 0.2588 & 0.2585 & 0.2591 & 0.2589 \\
\hline 232.1 & 0.2417 & 0.2436 & 0.2420 & 0.2424 & 0.2424 & 0.2435 & 0.2430 & 0.2433 & 0.2435 & 0.2441 & 0.2440 \\
\hline 235.1 & 0.2307 & 0.2326 & 0.2314 & 0.2315 & 0.2317 & 0.2319 & 0.2321 & 0.2325 & 0.2323 & 0.2327 & 0.2327 \\
\hline 238.1 & 0.2226 & 0.2248 & 0.2231 & 0.2228 & 0.2239 & 0.2235 & 0.2234 & 0.2242 & 0.2238 & 0.2242 & 0.2246 \\
\hline 241.1 & 0.2161 & 0.2184 & 0.2163 & 0.2166 & 0.2171 & 0.2172 & 0.2164 & 0.2176 & 0.2169 & 0.2179 & 0.2179 \\
\hline 244.1 & 0.2094 & 0.2108 & 0.2096 & 0.2103 & 0.2099 & 0.2112 & 0.2099 & 0.2109 & 0.2109 & 0.2114 & 0.2115 \\
\hline 247.1 & 0.2036 & 0.2047 & 0.2042 & 0.2042 & 0.2040 & 0.2051 & 0.2048 & 0.2049 & 0.2049 & 0.2053 & 0.2054 \\
\hline 250.1 & 0.1986 & 0.2005 & 0.1992 & 0.1987 & 0.1997 & 0.1990 & 0.1998 & 0.1999 & 0.2000 & 0.2000 & 0.2004 \\
\hline $\mathrm{CONC}$ & 0 & 0.1 & 0.2 & 0.3 & 0.4 & 0.5 & 0.6 & 0.7 & 0.8 & 0.9 & 1 \\
\hline $\begin{array}{l}\text { ABS at } \\
190 \mathrm{~nm}\end{array}$ & 1.0326 & 1.0451 & 1.0460 & 1.0491 & 1.0480 & 1.0534 & 1.0519 & 1.0532 & 1.0544 & 1.0576 & 1.0636 \\
\hline STDV & 0.0012 & 0.0006 & 0.0020 & 0.0007 & 0.0022 & 0.0013 & 0.0004 & 0.0013 & 0.0010 & 0.0019 & 0.0006 \\
\hline
\end{tabular}




\begin{tabular}{|c|c|c|c|c|c|c|c|c|c|c|c|}
\hline \multicolumn{12}{|c|}{ Magnafloc in filtered/diluted Rideau River Water (3.2 NTU) (2018-03-26) } \\
\hline \multicolumn{12}{|c|}{ In-line spectrophotometer (4mm path length) } \\
\hline $\begin{array}{c}\text { Wavelength } \\
(\mathrm{nm})\end{array}$ & $\begin{array}{c}0 \\
\mathrm{mg} / \mathrm{L}\end{array}$ & $\begin{array}{c}0.1 \\
\mathrm{mg} / \mathrm{L}\end{array}$ & $\begin{array}{c}0.2 \\
\mathrm{mg} / \mathrm{L}\end{array}$ & $\begin{array}{c}0.3 \\
\mathrm{mg} / \mathrm{L}\end{array}$ & $\begin{array}{c}0.4 \\
\mathrm{mg} / \mathrm{L}\end{array}$ & $\begin{array}{c}0.5 \\
\mathrm{mg} / \mathrm{L}\end{array}$ & $\begin{array}{c}0.6 \\
\mathrm{mg} / \mathrm{L}\end{array}$ & $\begin{array}{c}0.7 \\
\mathrm{mg} / \mathrm{L}\end{array}$ & $\begin{array}{c}0.8 \\
\mathrm{mg} / \mathrm{L}\end{array}$ & $\begin{array}{c}0.9 \\
\mathrm{mg} / \mathrm{L}\end{array}$ & $\begin{array}{c}1.0 \\
\mathrm{mg} / \mathrm{L}\end{array}$ \\
\hline 189.8 & 0.4756 & 0.4815 & 0.4858 & 0.4854 & 0.4828 & 0.4839 & 0.4829 & 0.4841 & 0.4836 & 0.4834 & 0.4886 \\
\hline 192.8 & 0.3908 & 0.3953 & 0.3982 & 0.3978 & 0.3944 & 0.3965 & 0.3949 & 0.3957 & 0.3968 & 0.3962 & 0.3997 \\
\hline 195.8 & 0.3542 & 0.3571 & 0.3591 & 0.3587 & 0.3561 & 0.3580 & 0.3560 & 0.3569 & 0.3587 & 0.3575 & 0.3605 \\
\hline 198.9 & 0.3228 & 0.3255 & 0.3272 & 0.3271 & 0.3249 & 0.3270 & 0.3250 & 0.3252 & 0.3264 & 0.3258 & 0.3283 \\
\hline 201.9 & 0.2984 & 0.3001 & 0.3036 & 0.3029 & 0.3008 & 0.3021 & 0.3009 & 0.3007 & 0.3010 & 0.3015 & 0.3033 \\
\hline 204.9 & 0.2710 & 0.2731 & 0.2751 & 0.2744 & 0.2730 & 0.2731 & 0.2733 & 0.2732 & 0.2731 & 0.2740 & 0.2753 \\
\hline 208 & 0.2427 & 0.2449 & 0.2458 & 0.2451 & 0.2433 & 0.2439 & 0.2438 & 0.2442 & 0.2445 & 0.2449 & 0.2469 \\
\hline 211 & 0.2170 & 0.2187 & 0.2194 & 0.2186 & 0.2167 & 0.2179 & 0.2178 & 0.2178 & 0.2191 & 0.2185 & 0.2207 \\
\hline 214 & 0.1929 & 0.1944 & 0.1947 & 0.1944 & 0.1930 & 0.1942 & 0.1937 & 0.1939 & 0.1949 & 0.1944 & 0.1963 \\
\hline 217 & 0.1698 & 0.1713 & 0.1725 & 0.1719 & 0.1710 & 0.1716 & 0.1714 & 0.1716 & 0.1717 & 0.1721 & 0.1734 \\
\hline 220 & 0.1556 & 0.1578 & 0.1584 & 0.1573 & 0.1571 & 0.1567 & 0.1574 & 0.1575 & 0.1571 & 0.1579 & 0.1590 \\
\hline 223.1 & 0.1421 & 0.1436 & 0.1443 & 0.1432 & 0.1430 & 0.1427 & 0.1433 & 0.1434 & 0.1438 & 0.1441 & 0.1452 \\
\hline 226.1 & 0.1327 & 0.1346 & 0.1340 & 0.1334 & 0.1328 & 0.1330 & 0.1335 & 0.1337 & 0.1347 & 0.1345 & 0.1356 \\
\hline 229.1 & 0.1243 & 0.1261 & 0.1250 & 0.1248 & 0.1241 & 0.1246 & 0.1249 & 0.1252 & 0.1259 & 0.1257 & 0.1268 \\
\hline 232.1 & 0.1171 & 0.1182 & 0.1184 & 0.1182 & 0.1176 & 0.1182 & 0.1183 & 0.1180 & 0.1182 & 0.1191 & 0.1196 \\
\hline 235.1 & 0.1116 & 0.1129 & 0.1134 & 0.1135 & 0.1130 & 0.1129 & 0.1130 & 0.1131 & 0.1128 & 0.1138 & 0.1145 \\
\hline 238.1 & 0.1078 & 0.1086 & 0.1094 & 0.1087 & 0.1084 & 0.1082 & 0.1091 & 0.1086 & 0.1091 & 0.1132 & 0.1103 \\
\hline 241.1 & 0.1035 & 0.1053 & 0.1050 & 0.1041 & 0.1038 & 0.1038 & 0.1048 & 0.1049 & 0.1055 & 0.1049 & 0.1063 \\
\hline 244.1 & 0.1011 & 0.1040 & 0.1024 & 0.1019 & 0.1013 & 0.1015 & 0.1022 & 0.1023 & 0.1031 & 0.1029 & 0.1035 \\
\hline 247.1 & 0.0991 & 0.1008 & 0.1003 & 0.1000 & 0.1000 & 0.1001 & 0.1005 & 0.1000 & 0.1004 & 0.1010 & 0.1010 \\
\hline 250.1 & 0.0971 & 0.0989 & 0.0991 & 0.0984 & 0.0983 & 0.0986 & 0.0988 & 0.0989 & 0.0981 & 0.0993 & 0.0999 \\
\hline $\mathrm{CONC}$ & 0 & 0.1 & 0.2 & 0.3 & 0.4 & 0.5 & 0.6 & 0.7 & 0.8 & 0.9 & 1 \\
\hline $\begin{array}{l}\text { ABS AT } \\
190 \mathrm{~nm}\end{array}$ & 0.4756 & 0.4815 & 0.4858 & 0.4854 & 0.4828 & 0.4839 & 0.4829 & 0.4841 & 0.4836 & 0.4834 & 0.4886 \\
\hline STDV & 0.0028 & 0.0000 & 0.0008 & 0.0004 & 0.0003 & 0.0008 & 0.0008 & 0.0009 & 0.0004 & 0.0015 & 0.0003 \\
\hline
\end{tabular}




\begin{tabular}{|c|c|c|c|c|c|c|c|c|c|c|c|}
\hline \multicolumn{12}{|c|}{ Magnafloc in filtered/diluted Rideau River Wat } \\
\hline \multicolumn{12}{|c|}{ Desktop spectrophotometer (1 cm path length) } \\
\hline $\begin{array}{l}\text { Wavelength } \\
\text { (nm) }\end{array}$ & $\begin{array}{c}0 \\
\mathrm{mg} / \mathrm{L}\end{array}$ & $\begin{array}{c}0.1 \\
\mathrm{mg} / \mathrm{L}\end{array}$ & $\begin{array}{c}0.2 \\
\mathrm{mg} / \mathrm{L}\end{array}$ & $\begin{array}{c}0.3 \\
\mathrm{mg} / \mathrm{L}\end{array}$ & $\begin{array}{c}0.4 \\
\mathrm{mg} / \mathrm{L}\end{array}$ & $\begin{array}{c}0.5 \\
\mathrm{mg} / \mathrm{L}\end{array}$ & $\begin{array}{c}0.6 \\
\mathrm{mg} / \mathrm{L}\end{array}$ & $\begin{array}{c}0.7 \\
\mathrm{mg} / \mathrm{L}\end{array}$ & $\begin{array}{c}0.8 \\
\mathrm{mg} / \mathrm{L}\end{array}$ & $\begin{array}{c}0.9 \\
\mathrm{mg} / \mathrm{L}\end{array}$ & $\begin{array}{c}1.0 \\
\mathrm{mg} / \mathrm{L}\end{array}$ \\
\hline 250 & 0.1502 & 0.1519 & 0.1532 & 0.1525 & 0.1523 & 0.1506 & 0.1509 & 0.1516 & 0.1513 & 0.1513 & 0.1524 \\
\hline 249 & 0.1548 & 0.1565 & 0.1579 & 0.1572 & 0.1569 & 0.1552 & 0.1557 & 0.1563 & 0.1558 & 0.1559 & 0.1570 \\
\hline 248 & 0.1595 & 0.1613 & 0.1624 & 0.1618 & 0.1617 & 0.1600 & 0.1603 & 0.1611 & 0.1607 & 0.1607 & 0.1619 \\
\hline 247 & 0.1641 & 0.1659 & 0.1672 & 0.1666 & 0.1663 & 0.1646 & 0.1649 & 0.1658 & 0.1653 & 0.1654 & 0.1665 \\
\hline 246 & 0.1685 & 0.1705 & 0.1719 & 0.1711 & 0.1707 & 0.1691 & 0.1694 & 0.1704 & 0.1700 & 0.1698 & 0.1711 \\
\hline 245 & 0.1729 & 0.1746 & 0.1760 & 0.1754 & 0.1752 & 0.1732 & 0.1738 & 0.1747 & 0.1741 & 0.1741 & 0.1753 \\
\hline 244 & 0.1769 & 0.1786 & 0.1800 & 0.1793 & 0.1792 & 0.1772 & 0.1777 & 0.1787 & 0.1781 & 0.1782 & 0.1794 \\
\hline 243 & 0.1805 & 0.1822 & 0.1837 & 0.1829 & 0.1829 & 0.1807 & 0.1812 & 0.1822 & 0.1817 & 0.1817 & 0.1830 \\
\hline 242 & 0.1835 & 0.1853 & 0.1868 & 0.1861 & 0.1858 & 0.1838 & 0.1844 & 0.1855 & 0.1849 & 0.1848 & 0.1860 \\
\hline 241 & 0.1863 & 0.1881 & 0.1896 & 0.1887 & 0.1887 & 0.1867 & 0.1871 & 0.1882 & 0.1876 & 0.1876 & 0.1888 \\
\hline 240 & 0.1887 & 0.1905 & 0.1919 & 0.1912 & 0.1911 & 0.1888 & 0.1895 & 0.1905 & 0.1899 & 0.1898 & 0.1912 \\
\hline 239 & 0.1908 & 0.1924 & 0.1939 & 0.1931 & 0.1930 & 0.1909 & 0.1914 & 0.1925 & 0.1919 & 0.1919 & 0.1931 \\
\hline 238 & 0.1924 & 0.1939 & 0.1956 & 0.1948 & 0.1947 & 0.1925 & 0.1930 & 0.1941 & 0.1935 & 0.1934 & 0.1947 \\
\hline 237 & 0.1938 & 0.1954 & 0.1971 & 0.1962 & 0.1962 & 0.1939 & 0.1943 & 0.1954 & 0.1947 & 0.1947 & 0.1962 \\
\hline 236 & 0.1949 & 0.1966 & 0.1984 & 0.1974 & 0.1973 & 0.1950 & 0.1955 & 0.1967 & 0.1960 & 0.1959 & 0.1973 \\
\hline 235 & 0.1962 & 0.1979 & 0.1995 & 0.1986 & 0.1986 & 0.1963 & 0.1967 & 0.1978 & 0.1972 & 0.1970 & 0.1985 \\
\hline 234 & 0.1975 & 0.1990 & 0.2008 & 0.1998 & 0.1998 & 0.1974 & 0.1979 & 0.1991 & 0.1984 & 0.1982 & 0.1998 \\
\hline 233 & 0.1988 & 0.2005 & 0.2023 & 0.2012 & 0.2011 & 0.1987 & 0.1993 & 0.2005 & 0.1998 & 0.1996 & 0.2011 \\
\hline 232 & 0.2002 & 0.2020 & 0.2039 & 0.2028 & 0.2029 & 0.2005 & 0.2010 & 0.2021 & 0.2013 & 0.2011 & 0.2029 \\
\hline 231 & 0.2022 & 0.2040 & 0.2059 & 0.2049 & 0.2047 & 0.2023 & 0.2028 & 0.2039 & 0.2032 & 0.2031 & 0.2047 \\
\hline 230 & 0.2045 & 0.2062 & 0.2083 & 0.2072 & 0.2071 & 0.2046 & 0.2051 & 0.2064 & 0.2056 & 0.2052 & 0.2070 \\
\hline 229 & 0.2071 & 0.2091 & 0.2112 & 0.2098 & 0.2098 & 0.2075 & 0.2078 & 0.2091 & 0.2083 & 0.2080 & 0.2099 \\
\hline 228 & 0.2102 & 0.2124 & 0.2144 & 0.2133 & 0.2131 & 0.2108 & 0.2111 & 0.2123 & 0.2115 & 0.2113 & 0.2132 \\
\hline 227 & 0.2139 & 0.2163 & 0.2184 & 0.2173 & 0.2170 & 0.2145 & 0.2148 & 0.2162 & 0.2155 & 0.2151 & 0.2171 \\
\hline 226 & 0.2186 & 0.2210 & 0.2230 & 0.2219 & 0.2216 & 0.2191 & 0.2194 & 0.2209 & 0.2200 & 0.2196 & 0.2217 \\
\hline 225 & 0.2237 & 0.2262 & 0.2282 & 0.2274 & 0.2269 & 0.2244 & 0.2246 & 0.2261 & 0.2252 & 0.2249 & 0.2271 \\
\hline 224 & 0.2297 & 0.2324 & 0.2345 & 0.2335 & 0.2330 & 0.2306 & 0.2308 & 0.2323 & 0.2314 & 0.2310 & 0.2332 \\
\hline 223 & 0.2366 & 0.2394 & 0.2416 & 0.2405 & 0.2400 & 0.2375 & 0.2377 & 0.2392 & 0.2384 & 0.2379 & 0.2402 \\
\hline 222 & 0.2444 & 0.2474 & 0.2496 & 0.2488 & 0.2479 & 0.2457 & 0.2456 & 0.2473 & 0.2465 & 0.2459 & 0.2483 \\
\hline 221 & 0.2531 & 0.2562 & 0.2584 & 0.2577 & 0.2567 & 0.2543 & 0.2545 & 0.2560 & 0.2552 & 0.2547 & 0.2571 \\
\hline 220 & 0.2628 & 0.2662 & 0.2686 & 0.2678 & 0.2668 & 0.2644 & 0.2644 & 0.2661 & 0.2653 & 0.2646 & 0.2671 \\
\hline 219 & 0.2735 & 0.2769 & 0.2793 & 0.2786 & 0.2775 & 0.2754 & 0.2751 & 0.2770 & 0.2760 & 0.2754 & 0.2780 \\
\hline 218 & 0.2855 & 0.2890 & 0.2914 & 0.2908 & 0.2894 & 0.2874 & 0.2871 & 0.2892 & 0.2883 & 0.2874 & 0.2900 \\
\hline 217 & 0.2977 & 0.3016 & 0.3041 & 0.3035 & 0.3018 & 0.2999 & 0.2996 & 0.3018 & 0.3007 & 0.2999 & 0.3026 \\
\hline 216 & 0.3112 & 0.3152 & 0.3176 & 0.3171 & 0.3156 & 0.3135 & 0.3131 & 0.3154 & 0.3144 & 0.3136 & 0.3163 \\
\hline
\end{tabular}




\begin{tabular}{|c|c|c|c|c|c|c|c|c|c|c|c|}
\hline 215 & 0.3250 & 0.3290 & 0.3317 & 0.3312 & 0.3293 & 0.3275 & 0.3270 & 0.3294 & 0.3282 & 0.3276 & 0.3302 \\
\hline 214 & 0.3396 & 0.3440 & 0.3465 & 0.3462 & 0.3441 & 0.3424 & 0.3419 & 0.3444 & 0.3431 & 0.3423 & 0.3451 \\
\hline 213 & 0.3545 & 0.3589 & 0.3616 & 0.3613 & 0.3591 & 0.3573 & 0.3567 & 0.3592 & 0.3579 & 0.3573 & 0.3598 \\
\hline 212 & 0.3698 & 0.3745 & 0.3772 & 0.3770 & 0.3746 & 0.3730 & 0.3721 & 0.3748 & 0.3736 & 0.3729 & 0.3755 \\
\hline 211 & 0.3853 & 0.3900 & 0.3929 & 0.3927 & 0.3902 & 0.3887 & 0.3876 & 0.3905 & 0.3890 & 0.3882 & 0.3910 \\
\hline 210 & 0.4009 & 0.4058 & 0.4088 & 0.4088 & 0.4061 & 0.4046 & 0.4034 & 0.4063 & 0.4050 & 0.4040 & 0.4069 \\
\hline 209 & 0.4167 & 0.4215 & 0.4247 & 0.4245 & 0.4217 & 0.4202 & 0.4189 & 0.4220 & 0.4205 & 0.4196 & 0.4224 \\
\hline 208 & 0.4323 & 0.4371 & 0.4406 & 0.4403 & 0.4375 & 0.4359 & 0.4345 & 0.4375 & 0.4362 & 0.4353 & 0.4383 \\
\hline 207 & 0.4476 & 0.4525 & 0.4561 & 0.4558 & 0.4529 & 0.4512 & 0.4498 & 0.4529 & 0.4513 & 0.4504 & 0.4535 \\
\hline 206 & 0.4632 & 0.4678 & 0.4717 & 0.4713 & 0.4684 & 0.4666 & 0.4651 & 0.4682 & 0.4667 & 0.4657 & 0.4689 \\
\hline 205 & 0.4784 & 0.4829 & 0.4871 & 0.4865 & 0.4837 & 0.4817 & 0.4800 & 0.4832 & 0.4817 & 0.4807 & 0.4839 \\
\hline 204 & 0.4941 & 0.4989 & 0.5031 & 0.5025 & 0.4996 & 0.4977 & 0.4960 & 0.4992 & 0.4976 & 0.4963 & 0.4999 \\
\hline 203 & 0.5097 & 0.5148 & 0.5193 & 0.5187 & 0.5156 & 0.5137 & 0.5116 & 0.5149 & 0.5133 & 0.5122 & 0.5158 \\
\hline 202 & 0.5266 & 0.5320 & 0.5367 & 0.5358 & 0.5327 & 0.5311 & 0.5288 & 0.5322 & 0.5305 & 0.5292 & 0.5332 \\
\hline 201 & 0.5447 & 0.5503 & 0.5554 & 0.5544 & 0.5510 & 0.5493 & 0.5470 & 0.5504 & 0.5487 & 0.5474 & 0.5516 \\
\hline 200 & 0.5650 & 0.5713 & 0.5766 & 0.5754 & 0.5722 & 0.5704 & 0.5680 & 0.5715 & 0.5698 & 0.5684 & 0.5730 \\
\hline 199 & 0.5879 & 0.5949 & 0.6006 & 0.5993 & 0.5959 & 0.5942 & 0.5918 & 0.5952 & 0.5934 & 0.5919 & 0.5974 \\
\hline 198 & 0.6146 & 0.6223 & 0.6282 & 0.6269 & 0.6232 & 0.6218 & 0.6190 & 0.6226 & 0.6207 & 0.6193 & 0.6254 \\
\hline 197 & 0.6460 & 0.6548 & 0.6608 & 0.6593 & 0.6554 & 0.6543 & 0.6515 & 0.6551 & 0.6532 & 0.6518 & 0.6584 \\
\hline 196 & 0.6832 & 0.6930 & 0.6995 & 0.6980 & 0.6934 & 0.6928 & 0.6897 & 0.6934 & 0.6916 & 0.6902 & 0.6975 \\
\hline 195 & 0.7300 & 0.7410 & 0.7479 & 0.7463 & 0.7410 & 0.7410 & 0.7380 & 0.7413 & 0.7393 & 0.7385 & 0.7466 \\
\hline 194 & 0.7848 & 0.7976 & 0.8042 & 0.8028 & 0.7967 & 0.7976 & 0.7937 & 0.7969 & 0.7956 & 0.7952 & 0.8036 \\
\hline 193 & 0.8524 & 0.8667 & 0.8737 & 0.8717 & 0.8654 & 0.8666 & 0.8626 & 0.8659 & 0.8642 & 0.8644 & 0.8737 \\
\hline 192 & 0.9438 & 0.9596 & 0.9668 & 0.9648 & 0.9571 & 0.9590 & 0.9549 & 0.9579 & 0.9563 & 0.9571 & 0.9676 \\
\hline 191 & 1.0645 & 1.0825 & 1.0897 & 1.0873 & 1.0785 & 1.0815 & 1.0766 & 1.0794 & 1.0778 & 1.0795 & 1.0908 \\
\hline 190 & 1.2094 & 1.2298 & 1.2369 & 1.2335 & 1.2237 & 1.2273 & 1.2222 & 1.2237 & 1.2231 & 1.2254 & 1.2376 \\
\hline $\mathrm{CONC}$ & 0 & 0.1 & 0.2 & 0.3 & 0.4 & 0.5 & 0.6 & 0.7 & 0.8 & 0.9 & 1 \\
\hline ABS AT 19NM & 1.2094 & 1.2298 & 1.2369 & 1.2335 & 1.2237 & 1.2273 & 1.2222 & 1.2237 & 1.2231 & 1.2254 & 1.2376 \\
\hline STDV & 0.0010 & 0.0009 & 0.0053 & 0.0035 & 0.0031 & 0.0042 & 0.0010 & 0.0037 & 0.0033 & 0.0017 & 0.0027 \\
\hline
\end{tabular}




\section{Appendix C: UV-vis absorbance data for experimental phase 3}

\begin{tabular}{|c|c|c|c|c|c|c|c|}
\hline \multicolumn{8}{|c|}{ Magnafloc in deionized water with Actiflo process chemicals (2018-05-08) } \\
\hline \multicolumn{8}{|c|}{ Desktop spectrophotometer (1 $\mathrm{cm}$ path length) } \\
\hline Wavelength $(\mathrm{nm})$ & $0 \mathrm{mg} / \mathrm{L}$ & $0.5 \mathrm{mg} / \mathrm{L}$ & $1.0 \mathrm{mg} / \mathrm{L}$ & $1.5 \mathrm{mg} / \mathrm{L}$ & $2.0 \mathrm{mg} / \mathrm{L}$ & $2.5 \mathrm{mg} / \mathrm{L}$ & $3.0 \mathrm{mg} / \mathrm{L}$ \\
\hline 250 & 0.1892 & 0.1892 & 0.1749 & 0.1669 & 0.1609 & 0.1551 & 0.1599 \\
\hline 249 & 0.1943 & 0.1939 & 0.1795 & 0.1714 & 0.1654 & 0.1594 & 0.1648 \\
\hline 248 & 0.1993 & 0.1986 & 0.1840 & 0.1758 & 0.1697 & 0.1635 & 0.1690 \\
\hline 247 & 0.2048 & 0.2034 & 0.1887 & 0.1803 & 0.1741 & 0.1679 & 0.1737 \\
\hline 246 & 0.2102 & 0.2081 & 0.1931 & 0.1844 & 0.1784 & 0.1721 & 0.1782 \\
\hline 245 & 0.2152 & 0.2124 & 0.1975 & 0.1885 & 0.1825 & 0.1761 & 0.1825 \\
\hline 244 & 0.2201 & 0.2162 & 0.2014 & 0.1918 & 0.1861 & 0.1797 & 0.1864 \\
\hline 243 & 0.2246 & 0.2199 & 0.2048 & 0.1952 & 0.1894 & 0.1831 & 0.1898 \\
\hline 242 & 0.2290 & 0.2232 & 0.2078 & 0.1978 & 0.1921 & 0.1859 & 0.1929 \\
\hline 241 & 0.2327 & 0.2258 & 0.2104 & 0.2000 & 0.1944 & 0.1883 & 0.1956 \\
\hline 240 & 0.2363 & 0.2282 & 0.2125 & 0.2017 & 0.1962 & 0.1904 & 0.1977 \\
\hline 239 & 0.2397 & 0.2302 & 0.2142 & 0.2029 & 0.1978 & 0.1920 & 0.1996 \\
\hline 238 & 0.2426 & 0.2316 & 0.2155 & 0.2038 & 0.1987 & 0.1931 & 0.2009 \\
\hline 237 & 0.2453 & 0.2327 & 0.2163 & 0.2043 & 0.1994 & 0.1939 & 0.2018 \\
\hline 236 & 0.2478 & 0.2336 & 0.2168 & 0.2045 & 0.1996 & 0.1945 & 0.2026 \\
\hline 235 & 0.2500 & 0.2344 & 0.2170 & 0.2043 & 0.1996 & 0.1947 & 0.2030 \\
\hline 234 & 0.2521 & 0.2346 & 0.2170 & 0.2040 & 0.1995 & 0.1949 & 0.2032 \\
\hline 233 & 0.2539 & 0.2349 & 0.2169 & 0.2037 & 0.1994 & 0.1950 & 0.2034 \\
\hline 232 & 0.2559 & 0.2353 & 0.2167 & 0.2033 & 0.1992 & 0.1949 & 0.2036 \\
\hline 231 & 0.2577 & 0.2354 & 0.2167 & 0.2029 & 0.1990 & 0.1948 & 0.2036 \\
\hline 230 & 0.2592 & 0.2358 & 0.2166 & 0.2027 & 0.1988 & 0.1949 & 0.2039 \\
\hline 229 & 0.2608 & 0.2360 & 0.2165 & 0.2023 & 0.1989 & 0.1949 & 0.2041 \\
\hline 228 & 0.2622 & 0.2364 & 0.2167 & 0.2024 & 0.1990 & 0.1950 & 0.2045 \\
\hline 227 & 0.2634 & 0.2368 & 0.2169 & 0.2025 & 0.1993 & 0.1954 & 0.2049 \\
\hline 226 & 0.2646 & 0.2373 & 0.2174 & 0.2030 & 0.1996 & 0.1959 & 0.2056 \\
\hline 225 & 0.2656 & 0.2381 & 0.2183 & 0.2035 & 0.2004 & 0.1965 & 0.2063 \\
\hline 224 & 0.2667 & 0.2391 & 0.2192 & 0.2046 & 0.2015 & 0.1972 & 0.2074 \\
\hline 223 & 0.2679 & 0.2402 & 0.2207 & 0.2060 & 0.2026 & 0.1986 & 0.2087 \\
\hline 222 & 0.2690 & 0.2420 & 0.2225 & 0.2079 & 0.2044 & 0.2001 & 0.2109 \\
\hline 221 & 0.2706 & 0.2439 & 0.2248 & 0.2101 & 0.2064 & 0.2021 & 0.2132 \\
\hline 220 & 0.2725 & 0.2463 & 0.2277 & 0.2132 & 0.2091 & 0.2046 & 0.2159 \\
\hline 219 & 0.2747 & 0.2494 & 0.2312 & 0.2167 & 0.2122 & 0.2076 & 0.2194 \\
\hline 218 & 0.2776 & 0.2530 & 0.2352 & 0.2207 & 0.2161 & 0.2112 & 0.2235 \\
\hline
\end{tabular}




\begin{tabular}{|c|c|c|c|c|c|c|c|}
\hline 217 & 0.2812 & 0.2572 & 0.2396 & 0.2254 & 0.2203 & 0.2152 & 0.2282 \\
\hline 216 & 0.2854 & 0.2619 & 0.2447 & 0.2306 & 0.2251 & 0.2200 & 0.2334 \\
\hline 215 & 0.2900 & 0.2672 & 0.2504 & 0.2361 & 0.2305 & 0.2251 & 0.2391 \\
\hline 214 & 0.2957 & 0.2731 & 0.2566 & 0.2423 & 0.2365 & 0.2310 & 0.2454 \\
\hline 213 & 0.3023 & 0.2798 & 0.2635 & 0.2490 & 0.2432 & 0.2374 & 0.2526 \\
\hline 212 & 0.3100 & 0.2873 & 0.2712 & 0.2564 & 0.2504 & 0.2444 & 0.2606 \\
\hline 211 & 0.3188 & 0.2955 & 0.2795 & 0.2642 & 0.2581 & 0.2522 & 0.2692 \\
\hline 210 & 0.3286 & 0.3045 & 0.2885 & 0.2726 & 0.2665 & 0.2605 & 0.2788 \\
\hline 209 & 0.3394 & 0.3142 & 0.2983 & 0.2814 & 0.2755 & 0.2695 & 0.2889 \\
\hline 208 & 0.3510 & 0.3245 & 0.3088 & 0.2907 & 0.2849 & 0.2792 & 0.2999 \\
\hline 207 & 0.3639 & 0.3356 & 0.3201 & 0.3005 & 0.2950 & 0.2896 & 0.3119 \\
\hline 206 & 0.3775 & 0.3474 & 0.3321 & 0.3109 & 0.3058 & 0.3004 & 0.3247 \\
\hline 205 & 0.3922 & 0.3607 & 0.3460 & 0.3226 & 0.3175 & 0.3125 & 0.3392 \\
\hline 204 & 0.4077 & 0.3742 & 0.3603 & 0.3345 & 0.3300 & 0.3252 & 0.3546 \\
\hline 203 & 0.4239 & 0.3889 & 0.3763 & 0.3475 & 0.3433 & 0.3388 & 0.3718 \\
\hline 202 & 0.4411 & 0.4048 & 0.3937 & 0.3616 & 0.3577 & 0.3537 & 0.3905 \\
\hline 201 & 0.4593 & 0.4223 & 0.4130 & 0.3771 & 0.3735 & 0.3698 & 0.4114 \\
\hline 200 & 0.4785 & 0.4410 & 0.4339 & 0.3938 & 0.3908 & 0.3875 & 0.4341 \\
\hline 199 & 0.4985 & 0.4610 & 0.4570 & 0.4121 & 0.4093 & 0.4063 & 0.4590 \\
\hline 198 & 0.5188 & 0.4819 & 0.4812 & 0.4315 & 0.4294 & 0.4262 & 0.4852 \\
\hline 197 & 0.5400 & 0.5046 & 0.5076 & 0.4527 & 0.4502 & 0.4477 & 0.5137 \\
\hline 196 & 0.5617 & 0.5280 & 0.5349 & 0.4748 & 0.4726 & 0.4699 & 0.5432 \\
\hline 195 & 0.5864 & 0.5551 & 0.5666 & 0.5011 & 0.4974 & 0.4960 & 0.5769 \\
\hline 194 & 0.6078 & 0.5791 & 0.5944 & 0.5246 & 0.5222 & 0.5191 & 0.6069 \\
\hline 193 & 0.6333 & 0.6078 & 0.6272 & 0.5530 & 0.5504 & 0.5472 & 0.6413 \\
\hline 192 & 0.6648 & 0.6421 & 0.6652 & 0.5875 & 0.5843 & 0.5809 & 0.6814 \\
\hline 191 & 0.7070 & 0.6873 & 0.7141 & 0.6331 & 0.6308 & 0.6258 & 0.7318 \\
\hline 190 & 0.7602 & 0.7426 & 0.7722 & 0.6892 & 0.6821 & 0.6817 & 0.7920 \\
\hline $\mathrm{CONC}$ & 0 & 0.5 & 1 & 1.5 & 2 & 2.5 & 3 \\
\hline ABS at $190 \mathrm{~nm}$ & 0.7602 & 0.7426 & 0.7722 & 0.6892 & 0.6821 & 0.6817 & 0.7920 \\
\hline STDV & 0.0013 & 0.0010 & 0.0006 & 0.0019 & 0.0035 & 0.0008 & 0.0017 \\
\hline
\end{tabular}




\begin{tabular}{|c|c|c|c|c|c|c|c|}
\hline \multicolumn{8}{|c|}{ Magnafloc in deionized water with Actiflo process chemicals (2018-05-08) } \\
\hline \multicolumn{8}{|c|}{ In-line spectrophotometer (4mm path length) } \\
\hline $\begin{array}{c}\text { Wavelength } \\
(\mathrm{nm})\end{array}$ & $0 \mathrm{mg} / \mathrm{L}$ & $0.5 \mathrm{mg} / \mathrm{L}$ & $1.0 \mathrm{mg} / \mathrm{L}$ & $1.5 \mathrm{mg} / \mathrm{L}$ & $2.0 \mathrm{mg} / \mathrm{L}$ & $2.5 \mathrm{mg} / \mathrm{L}$ & $3.0 \mathrm{mg} / \mathrm{L}$ \\
\hline 189.8 & 0.4596 & 0.5261 & 0.5835 & 0.5589 & 0.5846 & 0.6120 & 0.7226 \\
\hline 192.8 & 0.4060 & 0.4693 & 0.5177 & 0.5076 & 0.5342 & 0.5639 & 0.6541 \\
\hline 195.8 & 0.3743 & 0.4362 & 0.4851 & 0.4831 & 0.5114 & 0.5378 & 0.6194 \\
\hline 198.9 & 0.3441 & 0.4124 & 0.4586 & 0.4644 & 0.4928 & 0.5178 & 0.5889 \\
\hline 201.9 & 0.3280 & 0.3991 & 0.4429 & 0.4525 & 0.4795 & 0.5022 & 0.5671 \\
\hline 204.9 & 0.3177 & 0.3884 & 0.4310 & 0.4462 & 0.4703 & 0.4940 & 0.5573 \\
\hline 208 & 0.3098 & 0.3780 & 0.4187 & 0.4322 & 0.4598 & 0.4817 & 0.5427 \\
\hline 211 & 0.3086 & 0.3745 & 0.4119 & 0.4238 & 0.4509 & 0.4744 & 0.5327 \\
\hline 214 & 0.3062 & 0.3676 & 0.3998 & 0.4140 & 0.4414 & 0.4630 & 0.5195 \\
\hline 217 & 0.3035 & 0.3581 & 0.3917 & 0.4035 & 0.4315 & 0.4527 & 0.5038 \\
\hline 220 & 0.2981 & 0.3506 & 0.3824 & 0.3930 & 0.4209 & 0.4409 & 0.4935 \\
\hline 223.1 & 0.2876 & 0.3410 & 0.3693 & 0.3835 & 0.4087 & 0.4282 & 0.4777 \\
\hline 226.1 & 0.2747 & 0.3327 & 0.3585 & 0.3723 & 0.3963 & 0.4166 & 0.4681 \\
\hline 229.1 & 0.2609 & 0.3219 & 0.3543 & 0.3662 & 0.3890 & 0.4056 & 0.4573 \\
\hline 232.1 & 0.2522 & 0.3137 & 0.3443 & 0.3591 & 0.3825 & 0.3980 & 0.4477 \\
\hline 235.1 & 0.2445 & 0.3103 & 0.3389 & 0.3557 & 0.3774 & 0.3913 & 0.4421 \\
\hline 238.1 & 0.2372 & 0.3061 & 0.3349 & 0.3521 & 0.3726 & 0.3880 & 0.4366 \\
\hline 241.1 & 0.2337 & 0.3021 & 0.3339 & 0.3499 & 0.3716 & 0.3865 & 0.4339 \\
\hline 244.1 & 0.2297 & 0.3012 & 0.3336 & 0.3502 & 0.3727 & 0.3869 & 0.4341 \\
\hline 247.1 & 0.2280 & 0.2994 & 0.3339 & 0.3509 & 0.3755 & 0.3896 & 0.4349 \\
\hline 250.1 & 0.2255 & 0.3003 & 0.3356 & 0.3517 & 0.3800 & 0.3954 & 0.4363 \\
\hline CONC & 0 & 0.5 & 1 & 1.5 & 2 & 2.5 & 3 \\
\hline ABS at $190 \mathrm{~nm}$ & 0.4596 & 0.5261 & 0.5835 & 0.5589 & 0.5846 & 0.6120 & 0.7226 \\
\hline STDV & 0.0081 & 0.0021 & 0.0014 & 0.0009 & 0.0047 & 0.0033 & 0.0047 \\
\hline
\end{tabular}




\begin{tabular}{|c|c|c|c|c|c|c|c|c|c|c|c|}
\hline \multicolumn{12}{|c|}{ Magnafloc in deionized water with Actiflo process chemicals (2018-04-25) } \\
\hline \multicolumn{12}{|c|}{ Desktop spectrophotometer (1 $\mathrm{cm}$ path length) } \\
\hline $\begin{array}{l}\text { Wavelength } \\
(\mathrm{nm})\end{array}$ & $\begin{array}{c}0 \\
\mathrm{mg} / \mathrm{L}\end{array}$ & $\begin{array}{c}0.1 \\
\mathrm{mg} / \mathrm{L}\end{array}$ & $\begin{array}{c}0.2 \\
\mathrm{mg} / \mathrm{L}\end{array}$ & $\begin{array}{c}0.3 \\
\mathrm{mg} / \mathrm{L}\end{array}$ & $\begin{array}{c}0.4 \\
\mathrm{mg} / \mathrm{L}\end{array}$ & $\begin{array}{c}0.5 \\
\mathrm{mg} / \mathrm{L}\end{array}$ & $\begin{array}{c}0.6 \\
\mathrm{mg} / \mathrm{L}\end{array}$ & $\begin{array}{c}0.7 \\
\mathrm{mg} / \mathrm{L}\end{array}$ & $\begin{array}{c}0.8 \\
\mathrm{mg} / \mathrm{L}\end{array}$ & $\begin{array}{c}0.9 \\
\mathrm{mg} / \mathrm{L}\end{array}$ & $\begin{array}{c}1.0 \\
\mathrm{mg} / \mathrm{L}\end{array}$ \\
\hline 250 & 0.1647 & 0.1498 & 0.1541 & 0.1464 & 0.1370 & 0.1345 & 0.1240 & 0.1239 & 0.1365 & 0.1332 & 0.1248 \\
\hline 249 & 0.1674 & 0.1511 & 0.1551 & 0.1473 & 0.1384 & 0.1355 & 0.1245 & 0.1250 & 0.1374 & 0.1343 & 0.1256 \\
\hline 248 & 0.1701 & 0.1529 & 0.1568 & 0.1487 & 0.1403 & 0.1365 & 0.1253 & 0.1257 & 0.1386 & 0.1358 & 0.1265 \\
\hline 247 & 0.1732 & 0.1546 & 0.1584 & 0.1501 & 0.1423 & 0.1377 & 0.1266 & 0.1272 & 0.1398 & 0.1375 & 0.1275 \\
\hline 246 & 0.1764 & 0.1566 & 0.1598 & 0.1515 & 0.1445 & 0.1388 & 0.1275 & 0.1281 & 0.1412 & 0.1390 & 0.1285 \\
\hline 245 & 0.1799 & 0.1585 & 0.1615 & 0.1530 & 0.1466 & 0.1402 & 0.1285 & 0.1293 & 0.1426 & 0.1407 & 0.1298 \\
\hline 244 & 0.1835 & 0.1605 & 0.1630 & 0.1545 & 0.1491 & 0.1414 & 0.1298 & 0.1305 & 0.1441 & 0.1423 & 0.1308 \\
\hline 243 & 0.1873 & 0.1628 & 0.1647 & 0.1558 & 0.1515 & 0.1427 & 0.1308 & 0.1316 & 0.1453 & 0.1440 & 0.1321 \\
\hline 242 & 0.1917 & 0.1651 & 0.1662 & 0.1573 & 0.1543 & 0.1442 & 0.1318 & 0.1328 & 0.1467 & 0.1456 & 0.1332 \\
\hline 241 & 0.1963 & 0.1674 & 0.1678 & 0.1588 & 0.1571 & 0.1456 & 0.1329 & 0.1338 & 0.1479 & 0.1473 & 0.1344 \\
\hline 240 & 0.2012 & 0.1700 & 0.1696 & 0.1602 & 0.1603 & 0.1471 & 0.1342 & 0.1351 & 0.1495 & 0.1491 & 0.1355 \\
\hline 239 & 0.2065 & 0.1728 & 0.1713 & 0.1617 & 0.1635 & 0.1485 & 0.1353 & 0.1366 & 0.1510 & 0.1509 & 0.1368 \\
\hline 238 & 0.2120 & 0.1757 & 0.1730 & 0.1633 & 0.1668 & 0.1498 & 0.1365 & 0.1379 & 0.1525 & 0.1527 & 0.1381 \\
\hline 237 & 0.2178 & 0.1787 & 0.1746 & 0.1648 & 0.1706 & 0.1513 & 0.1377 & 0.1392 & 0.1540 & 0.1545 & 0.1393 \\
\hline 236 & 0.2237 & 0.1817 & 0.1765 & 0.1662 & 0.1740 & 0.1528 & 0.1389 & 0.1403 & 0.1553 & 0.1562 & 0.1406 \\
\hline 235 & 0.2296 & 0.1847 & 0.1783 & 0.1679 & 0.1777 & 0.1542 & 0.1400 & 0.1416 & 0.1571 & 0.1579 & 0.1418 \\
\hline 234 & 0.2357 & 0.1879 & 0.1800 & 0.1694 & 0.1813 & 0.1555 & 0.1411 & 0.1429 & 0.1584 & 0.1596 & 0.1429 \\
\hline 233 & 0.2417 & 0.1910 & 0.1816 & 0.1710 & 0.1851 & 0.1569 & 0.1423 & 0.1441 & 0.1599 & 0.1613 & 0.1442 \\
\hline 232 & 0.2476 & 0.1940 & 0.1833 & 0.1726 & 0.1887 & 0.1585 & 0.1435 & 0.1454 & 0.1614 & 0.1630 & 0.1455 \\
\hline 231 & 0.2533 & 0.1970 & 0.1852 & 0.1742 & 0.1921 & 0.1599 & 0.1449 & 0.1466 & 0.1629 & 0.1647 & 0.1470 \\
\hline 230 & 0.2587 & 0.1999 & 0.1867 & 0.1758 & 0.1955 & 0.1613 & 0.1461 & 0.1480 & 0.1642 & 0.1664 & 0.1483 \\
\hline 229 & 0.2638 & 0.2026 & 0.1885 & 0.1774 & 0.1985 & 0.1627 & 0.1473 & 0.1492 & 0.1655 & 0.1681 & 0.1496 \\
\hline 228 & 0.2683 & 0.2049 & 0.1901 & 0.1790 & 0.2014 & 0.1643 & 0.1486 & 0.1505 & 0.1669 & 0.1696 & 0.1509 \\
\hline 227 & 0.2721 & 0.2071 & 0.1917 & 0.1806 & 0.2040 & 0.1656 & 0.1498 & 0.1518 & 0.1684 & 0.1713 & 0.1522 \\
\hline 226 & 0.2754 & 0.2090 & 0.1935 & 0.1821 & 0.2060 & 0.1669 & 0.1510 & 0.1529 & 0.1697 & 0.1728 & 0.1533 \\
\hline 225 & 0.2781 & 0.2105 & 0.1949 & 0.1835 & 0.2077 & 0.1682 & 0.1521 & 0.1539 & 0.1711 & 0.1743 & 0.1546 \\
\hline 224 & 0.2800 & 0.2118 & 0.1965 & 0.1851 & 0.2092 & 0.1695 & 0.1533 & 0.1552 & 0.1723 & 0.1757 & 0.1558 \\
\hline 223 & 0.2813 & 0.2127 & 0.1982 & 0.1866 & 0.2101 & 0.1708 & 0.1543 & 0.1564 & 0.1736 & 0.1773 & 0.1569 \\
\hline 222 & 0.2822 & 0.2135 & 0.1999 & 0.1881 & 0.2108 & 0.1722 & 0.1556 & 0.1573 & 0.1749 & 0.1788 & 0.1582 \\
\hline 221 & 0.2825 & 0.2142 & 0.2016 & 0.1897 & 0.2112 & 0.1733 & 0.1568 & 0.1588 & 0.1764 & 0.1806 & 0.1594 \\
\hline 220 & 0.2827 & 0.2146 & 0.2035 & 0.1913 & 0.2115 & 0.1746 & 0.1580 & 0.1600 & 0.1780 & 0.1823 & 0.1608 \\
\hline 219 & 0.2828 & 0.2152 & 0.2052 & 0.1929 & 0.2118 & 0.1760 & 0.1593 & 0.1614 & 0.1794 & 0.1841 & 0.1621 \\
\hline 218 & 0.2831 & 0.2158 & 0.2073 & 0.1946 & 0.2121 & 0.1774 & 0.1608 & 0.1628 & 0.1812 & 0.1860 & 0.1637 \\
\hline 217 & 0.2831 & 0.2163 & 0.2094 & 0.1965 & 0.2124 & 0.1790 & 0.1622 & 0.1644 & 0.1830 & 0.1880 & 0.1651 \\
\hline 216 & 0.2839 & 0.2170 & 0.2113 & 0.1984 & 0.2129 & 0.1806 & 0.1637 & 0.1659 & 0.1847 & 0.1902 & 0.1669 \\
\hline
\end{tabular}




\begin{tabular}{|c|c|c|c|c|c|c|c|c|c|c|c|}
\hline 215 & 0.2849 & 0.2178 & 0.2135 & 0.2002 & 0.2136 & 0.1822 & 0.1653 & 0.1676 & 0.1868 & 0.1924 & 0.1684 \\
\hline 214 & 0.2869 & 0.2191 & 0.2159 & 0.2022 & 0.2147 & 0.1842 & 0.1668 & 0.1694 & 0.1887 & 0.1950 & 0.1701 \\
\hline 213 & 0.2897 & 0.2207 & 0.2184 & 0.2043 & 0.2163 & 0.1863 & 0.1687 & 0.1712 & 0.1908 & 0.1978 & 0.1722 \\
\hline 212 & 0.2937 & 0.2228 & 0.2209 & 0.2067 & 0.2189 & 0.1886 & 0.1705 & 0.1733 & 0.1934 & 0.2010 & 0.1742 \\
\hline 211 & 0.2990 & 0.2256 & 0.2241 & 0.2092 & 0.2219 & 0.1913 & 0.1730 & 0.1755 & 0.1963 & 0.2047 & 0.1768 \\
\hline 210 & 0.3056 & 0.2290 & 0.2273 & 0.2123 & 0.2259 & 0.1943 & 0.1753 & 0.1782 & 0.1997 & 0.2089 & 0.1795 \\
\hline 209 & 0.3140 & 0.2330 & 0.2311 & 0.2154 & 0.2308 & 0.1983 & 0.1780 & 0.1812 & 0.2032 & 0.2137 & 0.1826 \\
\hline 208 & 0.3235 & 0.2378 & 0.2354 & 0.2191 & 0.2366 & 0.2025 & 0.1811 & 0.1846 & 0.2074 & 0.2195 & 0.1860 \\
\hline 207 & 0.3348 & 0.2433 & 0.2403 & 0.2232 & 0.2434 & 0.2079 & 0.1844 & 0.1885 & 0.2126 & 0.2262 & 0.1902 \\
\hline 206 & 0.3475 & 0.2497 & 0.2456 & 0.2278 & 0.2513 & 0.2141 & 0.1885 & 0.1930 & 0.2184 & 0.2340 & 0.1949 \\
\hline 205 & 0.3619 & 0.2572 & 0.2523 & 0.2332 & 0.2601 & 0.2215 & 0.1929 & 0.1983 & 0.2253 & 0.2435 & 0.2006 \\
\hline 204 & 0.3778 & 0.2654 & 0.2596 & 0.2396 & 0.2705 & 0.2306 & 0.1984 & 0.2049 & 0.2339 & 0.2551 & 0.2074 \\
\hline 203 & 0.3956 & 0.2753 & 0.2689 & 0.2473 & 0.2823 & 0.2411 & 0.2048 & 0.2124 & 0.2436 & 0.2687 & 0.2154 \\
\hline 202 & 0.4147 & 0.2858 & 0.2790 & 0.2557 & 0.2948 & 0.2536 & 0.2121 & 0.2211 & 0.2551 & 0.2843 & 0.2245 \\
\hline 201 & 0.4354 & 0.2975 & 0.2907 & 0.2652 & 0.3088 & 0.2679 & 0.2202 & 0.2308 & 0.2683 & 0.3025 & 0.2352 \\
\hline 200 & 0.4574 & 0.3107 & 0.3040 & 0.2761 & 0.3242 & 0.2842 & 0.2301 & 0.2424 & 0.2833 & 0.3233 & 0.2475 \\
\hline 199 & 0.4805 & 0.3245 & 0.3189 & 0.2884 & 0.3406 & 0.3025 & 0.2410 & 0.2552 & 0.3002 & 0.3465 & 0.2614 \\
\hline 198 & 0.5039 & 0.3390 & 0.3345 & 0.3015 & 0.3576 & 0.3222 & 0.2527 & 0.2691 & 0.3185 & 0.3717 & 0.2762 \\
\hline 197 & 0.5281 & 0.3549 & 0.3522 & 0.3163 & 0.3757 & 0.3444 & 0.2666 & 0.2849 & 0.3388 & 0.3996 & 0.2939 \\
\hline 196 & 0.5526 & 0.3710 & 0.3713 & 0.3318 & 0.3939 & 0.3674 & 0.2803 & 0.3017 & 0.3604 & 0.4287 & 0.3107 \\
\hline 195 & 0.5760 & 0.3891 & 0.3926 & 0.3501 & 0.4140 & 0.3931 & 0.2961 & 0.3208 & 0.3848 & 0.4611 & 0.3303 \\
\hline 194 & 0.5991 & 0.4060 & 0.4128 & 0.3676 & 0.4325 & 0.4176 & 0.3134 & 0.3393 & 0.4079 & 0.4913 & 0.3509 \\
\hline 193 & 0.6232 & 0.4259 & 0.4361 & 0.3881 & 0.4535 & 0.4452 & 0.3332 & 0.3614 & 0.4343 & 0.5245 & 0.3740 \\
\hline 192 & 0.6517 & 0.4509 & 0.4651 & 0.4143 & 0.4798 & 0.4773 & 0.3582 & 0.3885 & 0.4659 & 0.5621 & 0.4025 \\
\hline 191 & 0.6909 & 0.4858 & 0.5032 & 0.4499 & 0.5153 & 0.5203 & 0.3961 & 0.4271 & 0.5086 & 0.6118 & 0.4433 \\
\hline 190 & 0.7315 & 0.5300 & 0.5495 & 0.4945 & 0.5592 & 0.5643 & 0.4315 & 0.4679 & 0.5524 & 0.6547 & 0.4809 \\
\hline $\mathrm{CONC}$ & 0 & 0.1 & 0.2 & 0.3 & 0.4 & 0.5 & 0.6 & 0.7 & 0.8 & 0.9 & 1 \\
\hline $\begin{array}{l}\text { ABS at } \\
190 \mathrm{~nm}\end{array}$ & 0.7315 & 0.5300 & 0.5495 & 0.4945 & 0.5592 & 0.5643 & 0.4315 & 0.4679 & 0.5524 & 0.6547 & 0.4809 \\
\hline STDV & 0.0033 & 0.0014 & 0.0011 & 0.0015 & 0.0013 & 0.0037 & 0.0018 & 0.0036 & 0.0039 & 0.0022 & 0.0012 \\
\hline
\end{tabular}




\begin{tabular}{|c|c|c|c|c|c|c|c|c|c|c|c|}
\hline \multicolumn{12}{|c|}{ Magnafloc in deionized water with Actiflo process chemicals (2018-04-25) } \\
\hline \multicolumn{12}{|c|}{ In-line spectrophotometer (4mm path length) } \\
\hline $\begin{array}{l}\text { Wavelength } \\
(\mathrm{nm})\end{array}$ & $0 \mathrm{mg} / \mathrm{L}$ & $\begin{array}{c}0.1 \\
\mathrm{mg} / \mathrm{L}\end{array}$ & $\begin{array}{c}0.2 \\
\mathrm{mg} / \mathrm{L}\end{array}$ & $\begin{array}{c}0.3 \\
\mathrm{mg} / \mathrm{L}\end{array}$ & $\begin{array}{c}0.4 \\
\mathrm{mg} / \mathrm{L}\end{array}$ & $\begin{array}{c}0.5 \\
\mathrm{mg} / \mathrm{L}\end{array}$ & $\begin{array}{c}0.6 \\
\mathrm{mg} / \mathrm{L}\end{array}$ & $\begin{array}{c}0.7 \\
\mathrm{mg} / \mathrm{L}\end{array}$ & $\begin{array}{c}0.8 \\
\mathrm{mg} / \mathrm{L}\end{array}$ & $\begin{array}{c}0.9 \\
\mathrm{mg} / \mathrm{L}\end{array}$ & $\begin{array}{c}1.0 \\
\mathrm{mg} / \mathrm{L}\end{array}$ \\
\hline 189.8 & 0.4821 & 0.3640 & 0.4128 & 0.4258 & 0.4974 & 0.5271 & 0.4624 & 0.4803 & 0.5395 & 0.6894 & 0.5579 \\
\hline 192.8 & 0.4119 & 0.3244 & 0.3675 & 0.3885 & 0.4550 & 0.4653 & 0.4289 & 0.4454 & 0.4884 & 0.6025 & 0.5146 \\
\hline 195.8 & 0.3622 & 0.3007 & 0.3414 & 0.3668 & 0.4278 & 0.4298 & 0.4095 & 0.4241 & 0.4596 & 0.5470 & 0.4891 \\
\hline 198.9 & 0.3170 & 0.2787 & 0.3196 & 0.3496 & 0.4039 & 0.4022 & 0.3944 & 0.4070 & 0.4387 & 0.5096 & 0.4705 \\
\hline 201.9 & 0.2846 & 0.2661 & 0.3074 & 0.3387 & 0.3876 & 0.3870 & 0.3882 & 0.3963 & 0.4247 & 0.4852 & 0.4570 \\
\hline 204.9 & 0.2650 & 0.2564 & 0.2958 & 0.3286 & 0.3748 & 0.3735 & 0.3811 & 0.3866 & 0.4142 & 0.4706 & 0.4473 \\
\hline 208 & 0.2564 & 0.2515 & 0.2870 & 0.3192 & 0.3674 & 0.3644 & 0.3714 & 0.3769 & 0.4048 & 0.4578 & 0.4399 \\
\hline 211 & 0.2582 & 0.2489 & 0.2800 & 0.3125 & 0.3622 & 0.3582 & 0.3632 & 0.3714 & 0.3955 & 0.4479 & 0.4308 \\
\hline 214 & 0.2588 & 0.2492 & 0.2725 & 0.3052 & 0.3586 & 0.3493 & 0.3538 & 0.3627 & 0.3870 & 0.4377 & 0.4214 \\
\hline 217 & 0.2592 & 0.2455 & 0.2655 & 0.2972 & 0.3492 & 0.3418 & 0.3455 & 0.3548 & 0.3780 & 0.4261 & 0.4102 \\
\hline 220 & 0.2553 & 0.2431 & 0.2600 & 0.2910 & 0.3444 & 0.3344 & 0.3388 & 0.3467 & 0.3687 & 0.4152 & 0.4015 \\
\hline 223.1 & 0.2462 & 0.2342 & 0.2543 & 0.2849 & 0.3321 & 0.3290 & 0.3337 & 0.3396 & 0.3603 & 0.4059 & 0.3920 \\
\hline 226.1 & 0.2336 & 0.2254 & 0.2484 & 0.2773 & 0.3185 & 0.3192 & 0.3245 & 0.3305 & 0.3513 & 0.3958 & 0.3827 \\
\hline 229.1 & 0.2171 & 0.2172 & 0.2423 & 0.2711 & 0.3067 & 0.3121 & 0.3197 & 0.3246 & 0.3446 & 0.3867 & 0.3737 \\
\hline 232.1 & 0.2013 & 0.2097 & 0.2366 & 0.2654 & 0.2934 & 0.3067 & 0.3124 & 0.3181 & 0.3377 & 0.3797 & 0.3672 \\
\hline 235.1 & 0.1874 & 0.2018 & 0.2320 & 0.2608 & 0.2867 & 0.3015 & 0.3073 & 0.3142 & 0.3329 & 0.3727 & 0.3628 \\
\hline 238.1 & 0.1776 & 0.1964 & 0.2288 & 0.2581 & 0.2792 & 0.2991 & 0.3046 & 0.3126 & 0.3303 & 0.3687 & 0.3589 \\
\hline 241.1 & 0.1660 & 0.1920 & 0.2252 & 0.2549 & 0.2721 & 0.2949 & 0.3010 & 0.3098 & 0.3266 & 0.3625 & 0.3557 \\
\hline 244.1 & 0.1609 & 0.1889 & 0.2233 & 0.2530 & 0.2689 & 0.2936 & 0.2994 & 0.3082 & 0.3251 & 0.3594 & 0.3553 \\
\hline 247.1 & 0.1579 & 0.1877 & 0.2236 & 0.2529 & 0.2668 & 0.2934 & 0.2998 & 0.3093 & 0.3259 & 0.3604 & 0.3562 \\
\hline 250.1 & 0.1540 & 0.1863 & 0.2222 & 0.2517 & 0.2672 & 0.2932 & 0.2987 & 0.3085 & 0.3268 & 0.3580 & 0.3559 \\
\hline $\mathrm{CONC}$ & 0 & 0.1 & 0.2 & 0.3 & 0.4 & 0.5 & 0.6 & 0.7 & 0.8 & 0.9 & 1 \\
\hline $\begin{array}{l}\text { ABS at } \\
190 \mathrm{~nm}\end{array}$ & 0.4821 & 0.3640 & 0.4128 & 0.4258 & 0.4974 & 0.5271 & 0.4624 & 0.4803 & 0.5395 & 0.6894 & 0.5579 \\
\hline STDV & 0.0221 & 0.0014 & 0.0162 & 0.0024 & 0.0164 & 0.0240 & 0.0177 & 0.0228 & 0.0221 & 0.0061 & 0.0175 \\
\hline
\end{tabular}




\begin{tabular}{|c|c|c|c|c|c|c|c|}
\hline \multicolumn{8}{|c|}{ Magnafloc in Rideau River water with Actiflo process chemicals (2018-04-11) } \\
\hline \multicolumn{8}{|c|}{ Desktop spectrophotometer (1 cm path length) } \\
\hline $\begin{array}{l}\text { Wavelength } \\
(\mathrm{nm})\end{array}$ & $0 \mathrm{mg} / \mathrm{L}$ & $0.5 \mathrm{mg} / \mathrm{L}$ & $1.0 \mathrm{mg} / \mathrm{L}$ & $1.5 \mathrm{mg} / \mathrm{L}$ & $2.0 \mathrm{mg} / \mathrm{L}$ & $2.5 \mathrm{mg} / \mathrm{L}$ & $3.0 \mathrm{mg} / \mathrm{L}$ \\
\hline 250 & 0.3813 & 0.2770 & 0.3372 & 0.3373 & 0.3479 & 0.3635 & 0.3312 \\
\hline 249 & 0.3837 & 0.2790 & 0.3396 & 0.3397 & 0.3502 & 0.3662 & 0.3335 \\
\hline 248 & 0.3865 & 0.2814 & 0.3419 & 0.3422 & 0.3526 & 0.3691 & 0.3361 \\
\hline 247 & 0.3893 & 0.2837 & 0.3446 & 0.3450 & 0.3555 & 0.3721 & 0.3386 \\
\hline 246 & 0.3922 & 0.2863 & 0.3475 & 0.3479 & 0.3586 & 0.3753 & 0.3416 \\
\hline 245 & 0.3953 & 0.2891 & 0.3505 & 0.3507 & 0.3619 & 0.3789 & 0.3446 \\
\hline 244 & 0.3985 & 0.2920 & 0.3534 & 0.3539 & 0.3654 & 0.3825 & 0.3477 \\
\hline 243 & 0.4023 & 0.2953 & 0.3571 & 0.3576 & 0.3693 & 0.3867 & 0.3514 \\
\hline 242 & 0.4063 & 0.2990 & 0.3610 & 0.3616 & 0.3736 & 0.3912 & 0.3552 \\
\hline 241 & 0.4107 & 0.3030 & 0.3651 & 0.3662 & 0.3783 & 0.3962 & 0.3595 \\
\hline 240 & 0.4156 & 0.3075 & 0.3699 & 0.3713 & 0.3835 & 0.4017 & 0.3643 \\
\hline 239 & 0.4209 & 0.3125 & 0.3752 & 0.3766 & 0.3891 & 0.4076 & 0.3697 \\
\hline 238 & 0.4267 & 0.3180 & 0.3809 & 0.3827 & 0.3954 & 0.4142 & 0.3754 \\
\hline 237 & 0.4330 & 0.3243 & 0.3872 & 0.3893 & 0.4022 & 0.4212 & 0.3815 \\
\hline 236 & 0.4400 & 0.3310 & 0.3942 & 0.3964 & 0.4095 & 0.4290 & 0.3885 \\
\hline 235 & 0.4474 & 0.3384 & 0.4017 & 0.4041 & 0.4175 & 0.4374 & 0.3958 \\
\hline 234 & 0.4557 & 0.3463 & 0.4098 & 0.4127 & 0.4264 & 0.4463 & 0.4041 \\
\hline 233 & 0.4648 & 0.3551 & 0.4189 & 0.4220 & 0.4358 & 0.4560 & 0.4130 \\
\hline 232 & 0.4745 & 0.3648 & 0.4287 & 0.4319 & 0.4462 & 0.4667 & 0.4229 \\
\hline 231 & 0.4851 & 0.3752 & 0.4393 & 0.4429 & 0.4573 & 0.4780 & 0.4334 \\
\hline 230 & 0.4964 & 0.3865 & 0.4506 & 0.4548 & 0.4691 & 0.4903 & 0.4450 \\
\hline 229 & 0.5089 & 0.3985 & 0.4629 & 0.4673 & 0.4819 & 0.5033 & 0.4573 \\
\hline 228 & 0.5221 & 0.4117 & 0.4762 & 0.4809 & 0.4956 & 0.5175 & 0.4707 \\
\hline 227 & 0.5367 & 0.4258 & 0.4907 & 0.4954 & 0.5102 & 0.5327 & 0.4852 \\
\hline 226 & 0.5523 & 0.4411 & 0.5062 & 0.5112 & 0.5261 & 0.5490 & 0.5010 \\
\hline 225 & 0.5693 & 0.4576 & 0.5228 & 0.5281 & 0.5431 & 0.5664 & 0.5178 \\
\hline 224 & 0.5875 & 0.4754 & 0.5411 & 0.5464 & 0.5614 & 0.5852 & 0.5364 \\
\hline 223 & 0.6072 & 0.4945 & 0.5607 & 0.5660 & 0.5809 & 0.6055 & 0.5562 \\
\hline 222 & 0.6287 & 0.5154 & 0.5819 & 0.5871 & 0.6022 & 0.6270 & 0.5777 \\
\hline 221 & 0.6515 & 0.5375 & 0.6045 & 0.6097 & 0.6246 & 0.6503 & 0.6006 \\
\hline 220 & 0.6759 & 0.5614 & 0.6289 & 0.6338 & 0.6488 & 0.6751 & 0.6253 \\
\hline 219 & 0.7018 & 0.5865 & 0.6545 & 0.6594 & 0.6740 & 0.7013 & 0.6511 \\
\hline 218 & 0.7291 & 0.6133 & 0.6816 & 0.6863 & 0.7011 & 0.7292 & 0.6788 \\
\hline 217 & 0.7573 & 0.6410 & 0.7098 & 0.7145 & 0.7291 & 0.7579 & 0.7075 \\
\hline 216 & 0.7867 & 0.6698 & 0.7392 & 0.7438 & 0.7581 & 0.7882 & 0.7373 \\
\hline
\end{tabular}




\begin{tabular}{|c|c|c|c|c|c|c|c|}
\hline 215 & 0.8168 & 0.6995 & 0.7691 & 0.7739 & 0.7881 & 0.8191 & 0.7679 \\
\hline 214 & 0.8480 & 0.7303 & 0.8005 & 0.8049 & 0.8191 & 0.8512 & 0.7996 \\
\hline 213 & 0.8794 & 0.7612 & 0.8320 & 0.8364 & 0.8506 & 0.8838 & 0.8316 \\
\hline 212 & 0.9114 & 0.7932 & 0.8639 & 0.8686 & 0.8828 & 0.9171 & 0.8642 \\
\hline 211 & 0.9433 & 0.8250 & 0.8962 & 0.9010 & 0.9152 & 0.9506 & 0.8969 \\
\hline 210 & 0.9756 & 0.8573 & 0.9289 & 0.9336 & 0.9482 & 0.9850 & 0.9297 \\
\hline 209 & 1.0078 & 0.8895 & 0.9614 & 0.9663 & 0.9812 & 1.0192 & 0.9625 \\
\hline 208 & 1.0403 & 0.9223 & 0.9944 & 0.9996 & 1.0147 & 1.0543 & 0.9958 \\
\hline 207 & 1.0729 & 0.9555 & 1.0276 & 1.0328 & 1.0485 & 1.0900 & 1.0293 \\
\hline 206 & 1.1063 & 0.9896 & 1.0618 & 1.0676 & 1.0833 & 1.1270 & 1.0636 \\
\hline 205 & 1.1399 & 1.0237 & 1.0965 & 1.1025 & 1.1192 & 1.1649 & 1.0986 \\
\hline 204 & 1.1747 & 1.0598 & 1.1325 & 1.1388 & 1.1564 & 1.2049 & 1.1346 \\
\hline 203 & 1.2108 & 1.0968 & 1.1698 & 1.1763 & 1.1957 & 1.2471 & 1.1725 \\
\hline 202 & 1.2492 & 1.1364 & 1.2100 & 1.2165 & 1.2374 & 1.2927 & 1.2131 \\
\hline 201 & 1.2898 & 1.1781 & 1.2526 & 1.2594 & 1.2818 & 1.3419 & 1.2563 \\
\hline 200 & 1.3341 & 1.2241 & 1.2994 & 1.3063 & 1.3308 & 1.3957 & 1.3039 \\
\hline 199 & 1.3826 & 1.2745 & 1.3500 & 1.3573 & 1.3841 & 1.4548 & 1.3563 \\
\hline 198 & 1.4368 & 1.3305 & 1.4070 & 1.4142 & 1.4433 & 1.5203 & 1.4149 \\
\hline 197 & 1.4986 & 1.3940 & 1.4716 & 1.4791 & 1.5105 & 1.5939 & 1.4817 \\
\hline 196 & 1.5719 & 1.4692 & 1.5476 & 1.5554 & 1.5886 & 1.6805 & 1.5611 \\
\hline 195 & 1.6495 & 1.5482 & 1.6268 & 1.6349 & 1.6744 & 1.7689 & 1.6435 \\
\hline 194 & 1.7453 & 1.6457 & 1.7255 & 1.7334 & 1.7733 & 1.8763 & 1.7454 \\
\hline 193 & 1.8536 & 1.7553 & 1.8346 & 1.8428 & 1.8847 & 1.9942 & 1.8579 \\
\hline 192 & 1.9859 & 1.8885 & 1.9674 & 1.9762 & 2.0201 & 2.1317 & 1.9952 \\
\hline 191 & 2.1499 & 2.0514 & 2.1300 & 2.1390 & 2.1833 & 2.2953 & 2.1608 \\
\hline 190 & 2.3269 & 2.2299 & 2.3052 & 2.3140 & 2.3562 & 2.4627 & 2.3364 \\
\hline $\mathrm{CONC}$ & 0 & 0.5 & 1 & 1.5 & 2 & 2.5 & 3 \\
\hline ABS AT $190 \mathrm{~nm}$ & 2.3269 & 2.2299 & 2.3052 & 2.3140 & 2.3562 & 2.4627 & 2.3364 \\
\hline STDV & 0.0010 & 0.0014 & 0.0011 & 0.0001 & 0.0021 & 0.0033 & 0.0048 \\
\hline
\end{tabular}




\begin{tabular}{|c|c|c|c|c|c|c|c|}
\hline \multicolumn{8}{|c|}{ Magnafloc in Rideau River water with Actiflo process chemicals (2018-04-11) } \\
\hline \multicolumn{8}{|c|}{ In-line spectrophotometer (4mm path length) } \\
\hline Wavelength $(\mathrm{nm})$ & $0 \mathrm{mg} / \mathrm{L}$ & $0.5 \mathrm{mg} / \mathrm{L}$ & $1.0 \mathrm{mg} / \mathrm{L}$ & $1.5 \mathrm{mg} / \mathrm{L}$ & $2.0 \mathrm{mg} / \mathrm{L}$ & $2.5 \mathrm{mg} / \mathrm{L}$ & $3.0 \mathrm{mg} / \mathrm{L}$ \\
\hline 189.8 & 1.2269 & 1.1044 & 1.1190 & 1.1445 & 1.1875 & 1.2565 & 1.3818 \\
\hline 192.8 & 1.0693 & 0.9443 & 0.9566 & 0.9827 & 1.0169 & 1.0801 & 1.1806 \\
\hline 195.8 & 0.9956 & 0.8743 & 0.8837 & 0.9071 & 0.9346 & 0.9962 & 1.0880 \\
\hline 198.9 & 0.9238 & 0.8010 & 0.8128 & 0.8355 & 0.8601 & 0.9150 & 1.0000 \\
\hline 201.9 & 0.8596 & 0.7363 & 0.7483 & 0.7712 & 0.7937 & 0.8496 & 0.9285 \\
\hline 204.9 & 0.7846 & 0.6637 & 0.6752 & 0.6961 & 0.7175 & 0.7714 & 0.8467 \\
\hline 208 & 0.7088 & 0.5912 & 0.6023 & 0.6227 & 0.6438 & 0.6941 & 0.7654 \\
\hline 211 & 0.6409 & 0.5254 & 0.5345 & 0.5572 & 0.5774 & 0.6267 & 0.6918 \\
\hline 214 & 0.5827 & 0.4687 & 0.4768 & 0.4985 & 0.5186 & 0.5652 & 0.6286 \\
\hline 217 & 0.5268 & 0.4154 & 0.4237 & 0.4445 & 0.4647 & 0.5086 & 0.5691 \\
\hline 220 & 0.4912 & 0.3810 & 0.3905 & 0.4105 & 0.4298 & 0.4729 & 0.5304 \\
\hline 223.1 & 0.4559 & 0.3468 & 0.3554 & 0.3741 & 0.3934 & 0.4353 & 0.4912 \\
\hline 226.1 & 0.4314 & 0.3238 & 0.3396 & 0.3502 & 0.3689 & 0.4082 & 0.4648 \\
\hline 229.1 & 0.4127 & 0.3048 & 0.3142 & 0.3315 & 0.3479 & 0.3880 & 0.4434 \\
\hline 232.1 & 0.3973 & 0.2913 & 0.2985 & 0.3146 & 0.3313 & 0.3716 & 0.4261 \\
\hline 235.1 & 0.3856 & 0.2793 & 0.2873 & 0.3041 & 0.3191 & 0.3583 & 0.4133 \\
\hline 238.1 & 0.3785 & 0.2728 & 0.2810 & 0.2971 & 0.3125 & 0.3517 & 0.4051 \\
\hline 241.1 & 0.3724 & 0.2672 & 0.2753 & 0.2898 & 0.3049 & 0.3452 & 0.3973 \\
\hline 244.1 & 0.3651 & 0.2622 & 0.2702 & 0.2853 & 0.2994 & 0.3400 & 0.3913 \\
\hline 247.1 & 0.3619 & 0.2570 & 0.2668 & 0.2820 & 0.2964 & 0.3371 & 0.3866 \\
\hline 250.1 & 0.3595 & 0.2553 & 0.2651 & 0.2787 & 0.2942 & 0.3357 & 0.3842 \\
\hline $\mathrm{CONC}$ & 0 & 0.5 & 1 & 1.5 & 2 & 2.5 & 3 \\
\hline ABS at $190 \mathrm{~nm}$ & 1.2269 & 1.1044 & 1.1190 & 1.1445 & 1.1875 & 1.2565 & 1.3818 \\
\hline STDV & 0.0222 & 0.0209 & 0.0142 & 0.0139 & 0.0060 & 0.0014 & 0.0062 \\
\hline
\end{tabular}




\begin{tabular}{|c|c|c|c|c|c|c|c|c|c|c|c|}
\hline \multicolumn{12}{|c|}{ Magnafloc in Rideau River water with Actiflo process chemicals (2018-04-30) } \\
\hline \multicolumn{12}{|c|}{ Desktop spectrophotometer (1 $\mathrm{cm}$ path length) } \\
\hline $\begin{array}{c}\text { Wavelength } \\
(\mathrm{nm})\end{array}$ & $\begin{array}{c}0 \\
\mathrm{mg} / \mathrm{L} \\
\end{array}$ & $\begin{array}{c}0.1 \\
\mathrm{mg} / \mathrm{L}\end{array}$ & $\begin{array}{c}0.2 \\
\mathrm{mg} / \mathrm{L}\end{array}$ & $\begin{array}{c}0.3 \\
\mathrm{mg} / \mathrm{L}\end{array}$ & $\begin{array}{c}0.4 \\
\mathrm{mg} / \mathrm{L} \\
\end{array}$ & $\begin{array}{c}0.5 \\
\mathrm{mg} / \mathrm{L} \\
\end{array}$ & $\begin{array}{c}0.6 \\
\mathrm{mg} / \mathrm{L} \\
\end{array}$ & $\begin{array}{c}0.7 \\
\mathrm{mg} / \mathrm{L} \\
\end{array}$ & $\begin{array}{c}0.8 \\
\mathrm{mg} / \mathrm{L}\end{array}$ & $\begin{array}{c}0.9 \\
\mathrm{mg} / \mathrm{L}\end{array}$ & $\begin{array}{c}1.0 \\
\mathrm{mg} / \mathrm{L}\end{array}$ \\
\hline 250 & 0.4355 & 0.3409 & 0.3250 & 0.2998 & 0.2938 & 0.3184 & 0.2773 & 0.2677 & 0.2908 & 0.2919 & 0.3126 \\
\hline 249 & 0.4405 & 0.3434 & 0.3271 & 0.3017 & 0.2957 & 0.3206 & 0.2794 & 0.2695 & 0.2929 & 0.2937 & 0.3148 \\
\hline 248 & 0.4458 & 0.3457 & 0.3293 & 0.3037 & 0.2978 & 0.3230 & 0.2813 & 0.2715 & 0.2949 & 0.2954 & 0.3166 \\
\hline 247 & 0.4517 & 0.3482 & 0.3316 & 0.3056 & 0.3000 & 0.3254 & 0.2832 & 0.2735 & 0.2970 & 0.2974 & 0.3189 \\
\hline 246 & 0.4583 & 0.3510 & 0.3341 & 0.3079 & 0.3023 & 0.3281 & 0.2856 & 0.2758 & 0.2995 & 0.2996 & 0.3213 \\
\hline 245 & 0.4658 & 0.3539 & 0.3367 & 0.3104 & 0.3048 & 0.3310 & 0.2880 & 0.2784 & 0.3021 & 0.3021 & 0.3238 \\
\hline 244 & 0.4741 & 0.3569 & 0.3395 & 0.3131 & 0.3075 & 0.3340 & 0.2905 & 0.2806 & 0.3047 & 0.3044 & 0.3265 \\
\hline 243 & 0.4831 & 0.3603 & 0.3427 & 0.3158 & 0.3105 & 0.3373 & 0.2932 & 0.2834 & 0.3076 & 0.3072 & 0.3295 \\
\hline 242 & 0.4931 & 0.3638 & 0.3458 & 0.3187 & 0.3136 & 0.3407 & 0.2960 & 0.2864 & 0.3108 & 0.3103 & 0.3326 \\
\hline 241 & 0.5041 & 0.3673 & 0.3495 & 0.3222 & 0.3170 & 0.3445 & 0.2993 & 0.2897 & 0.3143 & 0.3135 & 0.3361 \\
\hline 240 & 0.5161 & 0.3717 & 0.3534 & 0.3258 & 0.3208 & 0.3485 & 0.3029 & 0.2933 & 0.3180 & 0.3175 & 0.3399 \\
\hline 239 & 0.5294 & 0.3761 & 0.3578 & 0.3299 & 0.3250 & 0.3531 & 0.3069 & 0.2973 & 0.3223 & 0.3214 & 0.3440 \\
\hline 238 & 0.5433 & 0.3811 & 0.3624 & 0.3344 & 0.3295 & 0.3579 & 0.3110 & 0.3017 & 0.3269 & 0.3261 & 0.3486 \\
\hline 237 & 0.5584 & 0.3863 & 0.3676 & 0.3393 & 0.3346 & 0.3632 & 0.3158 & 0.3065 & 0.3320 & 0.3311 & 0.3536 \\
\hline 236 & 0.5740 & 0.3922 & 0.3732 & 0.3447 & 0.3402 & 0.3690 & 0.3211 & 0.3118 & 0.3374 & 0.3366 & 0.3592 \\
\hline 235 & 0.5908 & 0.3987 & 0.3796 & 0.3507 & 0.3462 & 0.3754 & 0.3271 & 0.3178 & 0.3435 & 0.3428 & 0.3653 \\
\hline 234 & 0.6081 & 0.4058 & 0.3865 & 0.3574 & 0.3529 & 0.3825 & 0.3336 & 0.3244 & 0.3502 & 0.3495 & 0.3721 \\
\hline 233 & 0.6264 & 0.4138 & 0.3942 & 0.3649 & 0.3604 & 0.3904 & 0.3410 & 0.3320 & 0.3579 & 0.3570 & 0.3797 \\
\hline 232 & 0.6451 & 0.4224 & 0.4028 & 0.3732 & 0.3690 & 0.3990 & 0.3492 & 0.3403 & 0.3662 & 0.3655 & 0.3880 \\
\hline 231 & 0.6640 & 0.4321 & 0.4124 & 0.3826 & 0.3784 & 0.4087 & 0.3583 & 0.3495 & 0.3757 & 0.3749 & 0.3973 \\
\hline 230 & 0.6834 & 0.4429 & 0.4231 & 0.3930 & 0.3891 & 0.4195 & 0.3686 & 0.3599 & 0.3860 & 0.3855 & 0.4078 \\
\hline 229 & 0.7028 & 0.4551 & 0.4351 & 0.4046 & 0.4008 & 0.4315 & 0.3801 & 0.3716 & 0.3977 & 0.3972 & 0.4198 \\
\hline 228 & 0.7223 & 0.4683 & 0.4483 & 0.4176 & 0.4137 & 0.4448 & 0.3928 & 0.3845 & 0.4107 & 0.4102 & 0.4330 \\
\hline 227 & 0.7419 & 0.4833 & 0.4629 & 0.4319 & 0.4283 & 0.4597 & 0.4069 & 0.3987 & 0.4253 & 0.4247 & 0.4475 \\
\hline 226 & 0.7612 & 0.4993 & 0.4791 & 0.4479 & 0.4442 & 0.4756 & 0.4226 & 0.4146 & 0.4412 & 0.4405 & 0.4635 \\
\hline 225 & 0.7806 & 0.5173 & 0.4970 & 0.4653 & 0.4619 & 0.4937 & 0.4400 & 0.4319 & 0.4589 & 0.4581 & 0.4811 \\
\hline 224 & 0.8000 & 0.5368 & 0.5162 & 0.4845 & 0.4812 & 0.5131 & 0.4588 & 0.4510 & 0.4780 & 0.4774 & 0.5004 \\
\hline 223 & 0.8195 & 0.5580 & 0.5375 & 0.5054 & 0.5023 & 0.5343 & 0.4798 & 0.4718 & 0.4990 & 0.4983 & 0.5214 \\
\hline 222 & 0.8394 & 0.5809 & 0.5603 & 0.5280 & 0.5248 & 0.5572 & 0.5020 & 0.4944 & 0.5217 & 0.5208 & 0.5442 \\
\hline 221 & 0.8598 & 0.6056 & 0.5850 & 0.5524 & 0.5493 & 0.5819 & 0.5263 & 0.5186 & 0.5461 & 0.5454 & 0.5686 \\
\hline 220 & 0.8807 & 0.6317 & 0.6112 & 0.5783 & 0.5752 & 0.6079 & 0.5519 & 0.5445 & 0.5719 & 0.5713 & 0.5944 \\
\hline 219 & 0.9027 & 0.6595 & 0.6389 & 0.6058 & 0.6027 & 0.6356 & 0.5792 & 0.5717 & 0.5997 & 0.5987 & 0.6220 \\
\hline 218 & 0.9255 & 0.6885 & 0.6679 & 0.6345 & 0.6313 & 0.6646 & 0.6077 & 0.6004 & 0.6285 & 0.6274 & 0.6505 \\
\hline 217 & 0.9496 & 0.7185 & 0.6978 & 0.6644 & 0.6611 & 0.6948 & 0.6374 & 0.6301 & 0.6584 & 0.6573 & 0.6805 \\
\hline 216 & 0.9743 & 0.7491 & 0.7281 & 0.6943 & 0.6912 & 0.7255 & 0.6675 & 0.6603 & 0.6890 & 0.6878 & 0.7109 \\
\hline 215 & 1.0007 & 0.7802 & 0.7588 & 0.7249 & 0.7219 & 0.7567 & 0.6979 & 0.6909 & 0.7198 & 0.7184 & 0.7418 \\
\hline
\end{tabular}




\begin{tabular}{|c|c|c|c|c|c|c|c|c|c|c|c|}
\hline 214 & 1.0279 & 0.8110 & 0.7897 & 0.7554 & 0.7529 & 0.7879 & 0.7282 & 0.7215 & 0.7508 & 0.7493 & 0.7728 \\
\hline 213 & 1.0570 & 0.8419 & 0.8207 & 0.7859 & 0.7836 & 0.8193 & 0.7590 & 0.7521 & 0.7818 & 0.7799 & 0.8040 \\
\hline 212 & 1.0871 & 0.8721 & 0.8506 & 0.8157 & 0.8137 & 0.8501 & 0.7888 & 0.7820 & 0.8125 & 0.8100 & 0.8345 \\
\hline 211 & 1.1186 & 0.9019 & 0.8800 & 0.8448 & 0.8429 & 0.8803 & 0.8184 & 0.8115 & 0.8423 & 0.8397 & 0.8642 \\
\hline 210 & 1.1515 & 0.9300 & 0.9081 & 0.8728 & 0.8711 & 0.9096 & 0.8463 & 0.8396 & 0.8715 & 0.8680 & 0.8929 \\
\hline 209 & 1.1855 & 0.9571 & 0.9354 & 0.8995 & 0.8981 & 0.9383 & 0.8737 & 0.8667 & 0.8998 & 0.8956 & 0.9202 \\
\hline 208 & 1.2207 & 0.9827 & 0.9608 & 0.9249 & 0.9236 & 0.9658 & 0.8995 & 0.8923 & 0.9268 & 0.9217 & 0.9465 \\
\hline 207 & 1.2571 & 1.0070 & 0.9850 & 0.9492 & 0.9478 & 0.9926 & 0.9242 & 0.9169 & 0.9532 & 0.9470 & 0.9718 \\
\hline 206 & 1.2938 & 1.0303 & 1.0082 & 0.9719 & 0.9706 & 1.0187 & 0.9480 & 0.9401 & 0.9786 & 0.9713 & 0.9958 \\
\hline 205 & 1.3326 & 1.0528 & 1.0308 & 0.9944 & 0.9934 & 1.0453 & 0.9715 & 0.9633 & 1.0047 & 0.9952 & 1.0194 \\
\hline 204 & 1.3715 & 1.0746 & 1.0528 & 1.0161 & 1.0152 & 1.0722 & 0.9946 & 0.9859 & 1.0301 & 1.0192 & 1.0424 \\
\hline 203 & 1.4126 & 1.0968 & 1.0748 & 1.0378 & 1.0372 & 1.1004 & 1.0184 & 1.0089 & 1.0575 & 1.0437 & 1.0664 \\
\hline 202 & 1.4547 & 1.1196 & 1.0974 & 1.0606 & 1.0602 & 1.1302 & 1.0433 & 1.0331 & 1.0856 & 1.0697 & 1.0913 \\
\hline 201 & 1.4993 & 1.1440 & 1.1222 & 1.0852 & 1.0849 & 1.1631 & 1.0702 & 1.0592 & 1.1171 & 1.0980 & 1.1188 \\
\hline 200 & 1.5470 & 1.1713 & 1.1498 & 1.1127 & 1.1127 & 1.2001 & 1.1007 & 1.0886 & 1.1519 & 1.1296 & 1.1494 \\
\hline 199 & 1.5981 & 1.2028 & 1.1815 & 1.1446 & 1.1447 & 1.2427 & 1.1357 & 1.1221 & 1.1924 & 1.1665 & 1.1849 \\
\hline 198 & 1.6521 & 1.2393 & 1.2181 & 1.1813 & 1.1828 & 1.2909 & 1.1762 & 1.1612 & 1.2384 & 1.2084 & 1.2271 \\
\hline 197 & 1.7114 & 1.2846 & 1.2634 & 1.2267 & 1.2276 & 1.3487 & 1.2256 & 1.2088 & 1.2938 & 1.2595 & 1.2746 \\
\hline 196 & 1.7770 & 1.3377 & 1.3171 & 1.2806 & 1.2822 & 1.4151 & 1.2833 & 1.2648 & 1.3574 & 1.3193 & 1.3325 \\
\hline 195 & 1.8563 & 1.4087 & 1.3879 & 1.3514 & 1.3509 & 1.4995 & 1.3587 & 1.3387 & 1.4396 & 1.3961 & 1.4026 \\
\hline 194 & 1.9373 & 1.4856 & 1.4655 & 1.4297 & 1.4318 & 1.5874 & 1.4398 & 1.4181 & 1.5261 & 1.4787 & 1.4895 \\
\hline 193 & 2.0351 & 1.5843 & 1.5642 & 1.5279 & 1.5311 & 1.6973 & 1.5425 & 1.5190 & 1.6336 & 1.5828 & 1.5925 \\
\hline 192 & 2.1558 & 1.7085 & 1.6894 & 1.6537 & 1.6571 & 1.8296 & 1.6702 & 1.6458 & 1.7655 & 1.7119 & 1.7211 \\
\hline 191 & 2.3085 & 1.8717 & 1.8537 & 1.8171 & 1.8250 & 1.9977 & 1.8359 & 1.8113 & 1.9331 & 1.8786 & 1.8948 \\
\hline 190 & 2.4698 & 2.0563 & 2.0376 & 2.0027 & 1.9995 & 2.1781 & 2.0204 & 1.9954 & 2.1158 & 2.0626 & 2.0586 \\
\hline $\mathrm{CONC}$ & 0 & 0.1 & 0.2 & 0.3 & 0.4 & 0.5 & 0.6 & 0.7 & 0.8 & 0.9 & 1 \\
\hline $\begin{array}{l}\text { ABS at } \\
190 \mathrm{~nm}\end{array}$ & 2.4698 & 2.0563 & 2.0376 & 2.0027 & 1.9995 & 2.1781 & 2.0204 & 1.9954 & 2.1158 & 2.0626 & 2.0586 \\
\hline STDV & 0.0006 & 0.0006 & 0.0013 & 0.0004 & 0.0101 & 0.0009 & 0.0011 & 0.0002 & 0.0040 & 0.0033 & 0.0022 \\
\hline
\end{tabular}




\begin{tabular}{|c|c|c|c|c|c|c|c|c|c|c|c|}
\hline \multicolumn{12}{|c|}{ Magnafloc in Rideau River water with Actiflo } \\
\hline \multicolumn{12}{|c|}{ In-line spectrophotometer (4mm path length) } \\
\hline $\begin{array}{l}\text { Wavelength } \\
(\mathrm{nm})\end{array}$ & $0 \mathrm{mg} / \mathrm{L}$ & $\begin{array}{c}0.1 \\
\mathrm{mg} / \mathrm{L}\end{array}$ & $\begin{array}{c}0.2 \\
\mathrm{mg} / \mathrm{L}\end{array}$ & $\begin{array}{c}0.3 \\
\mathrm{mg} / \mathrm{L}\end{array}$ & $\begin{array}{c}0.4 \\
\mathrm{mg} / \mathrm{L}\end{array}$ & $\begin{array}{c}0.5 \\
\mathrm{mg} / \mathrm{L}\end{array}$ & $\begin{array}{c}0.6 \\
\mathrm{mg} / \mathrm{L}\end{array}$ & $\begin{array}{c}0.7 \\
\mathrm{mg} / \mathrm{L}\end{array}$ & $\begin{array}{c}0.8 \\
\mathrm{mg} / \mathrm{L}\end{array}$ & $\begin{array}{c}0.9 \\
\mathrm{mg} / \mathrm{L}\end{array}$ & $\begin{array}{c}1.0 \\
\mathrm{mg} / \mathrm{L}\end{array}$ \\
\hline 189.8 & 1.9749 & 1.4671 & 1.4476 & 1.3916 & 1.4135 & 1.6155 & 1.5992 & 1.5924 & 1.7580 & 1.8040 & 1.8432 \\
\hline 192.8 & 1.6527 & 1.2702 & 1.2544 & 1.2035 & 1.2191 & 1.3828 & 1.3854 & 1.3809 & 1.5226 & 1.5720 & 1.6127 \\
\hline 195.8 & 1.4881 & 1.1783 & 1.1654 & 1.1169 & 1.1316 & 1.2759 & 1.2902 & 1.2951 & 1.4130 & 1.4711 & 1.5117 \\
\hline 198.9 & 1.3186 & 1.0852 & 1.0723 & 1.0245 & 1.0381 & 1.1619 & 1.1920 & 1.2002 & 1.3076 & 1.3675 & 1.4109 \\
\hline 201.9 & 1.1873 & 0.9973 & 0.9844 & 0.9383 & 0.9531 & 1.0696 & 1.0997 & 1.1107 & 1.2134 & 1.2734 & 1.3176 \\
\hline 204.9 & 1.0610 & 0.8882 & 0.8776 & 0.8352 & 0.8460 & 0.9612 & 0.9915 & 1.0033 & 1.0996 & 1.1602 & 1.2035 \\
\hline 208 & 0.9567 & 0.7797 & 0.7695 & 0.7264 & 0.7392 & 0.8465 & 0.8800 & 0.8934 & 0.9874 & 1.0465 & 1.0864 \\
\hline 211 & 0.8746 & 0.6827 & 0.6714 & 0.6302 & 0.6409 & 0.7465 & 0.7789 & 0.7908 & 0.8832 & 0.9414 & 0.9803 \\
\hline 214 & 0.8106 & 0.5997 & 0.5887 & 0.5490 & 0.5589 & 0.6644 & 0.6927 & 0.7058 & 0.7950 & 0.8517 & 0.8916 \\
\hline 217 & 0.7425 & 0.5219 & 0.5118 & 0.4732 & 0.4833 & 0.5838 & 0.6118 & 0.6266 & 0.7130 & 0.7665 & 0.8064 \\
\hline 220 & 0.6931 & 0.4736 & 0.4649 & 0.4266 & 0.4358 & 0.5329 & 0.5616 & 0.5756 & 0.6591 & 0.7120 & 0.7529 \\
\hline 223.1 & 0.6320 & 0.4273 & 0.4176 & 0.3804 & 0.3896 & 0.4858 & 0.5113 & 0.5244 & 0.6082 & 0.6590 & 0.6993 \\
\hline 226.1 & 0.5789 & 0.3978 & 0.3890 & 0.3513 & 0.3616 & 0.4552 & 0.4802 & 0.4946 & 0.5732 & 0.6234 & 0.6648 \\
\hline 229.1 & 0.5247 & 0.3744 & 0.3660 & 0.3289 & 0.3379 & 0.4298 & 0.4536 & 0.4678 & 0.5471 & 0.5942 & 0.6349 \\
\hline 232.1 & 0.4815 & 0.3566 & 0.3490 & 0.3136 & 0.3215 & 0.4126 & 0.4373 & 0.4505 & 0.5296 & 0.5760 & 0.6167 \\
\hline 235.1 & 0.4462 & 0.3447 & 0.3372 & 0.3030 & 0.3105 & 0.4007 & 0.4251 & 0.4387 & 0.5162 & 0.5619 & 0.6019 \\
\hline 238.1 & 0.4193 & 0.3360 & 0.3285 & 0.2936 & 0.3011 & 0.3908 & 0.4177 & 0.4294 & 0.5083 & 0.5532 & 0.5920 \\
\hline 241.1 & 0.3988 & 0.3289 & 0.3233 & 0.2871 & 0.2950 & 0.3860 & 0.4130 & 0.4235 & 0.5039 & 0.5494 & 0.5872 \\
\hline 244.1 & 0.3849 & 0.3232 & 0.3175 & 0.2823 & 0.2902 & 0.3811 & 0.4086 & 0.4193 & 0.4991 & 0.5455 & 0.5840 \\
\hline 247.1 & 0.3715 & 0.3176 & 0.3121 & 0.2789 & 0.2841 & 0.3766 & 0.4068 & 0.4173 & 0.4953 & 0.5402 & 0.5804 \\
\hline 250.1 & 0.3640 & 0.3130 & 0.3077 & 0.2736 & 0.2800 & 0.3723 & 0.4012 & 0.4128 & 0.4917 & 0.5395 & 0.5764 \\
\hline CONC & 0 & 0.1 & 0.2 & 0.3 & 0.4 & 0.5 & 0.6 & 0.7 & 0.8 & 0.9 & 1 \\
\hline $\begin{array}{l}\text { ABS at } \\
190 \mathrm{~nm}\end{array}$ & 0.2040 & 1.4671 & 1.4476 & 1.3916 & 1.4135 & 1.6155 & 1.5992 & 1.5924 & 1.7580 & 1.8040 & 1.8432 \\
\hline STDV & 1.9749 & 0.0190 & 0.0138 & 0.0100 & 0.0200 & 0.0027 & 0.0089 & 0.0145 & 0.0132 & 0.0107 & 0.0128 \\
\hline
\end{tabular}


Appendix D: $U V$-vis absorbance data for experimental phase 4

\begin{tabular}{|c|c|c|c|c|c|c|c|c|c|}
\hline \multicolumn{10}{|c|}{ Magnafloc determination of optimum dose in high turbidity Rideau River water (9172 NTU) } \\
\hline $\begin{array}{l}\text { Wavelength } \\
(\mathrm{nm})\end{array}$ & $0 \mathrm{mg} / \mathrm{L}$ & $0.1 \mathrm{mg} / \mathrm{L}$ & $0.5 \mathrm{mg} / \mathrm{L}$ & $1.0 \mathrm{mg} / \mathrm{L}$ & $1.5 \mathrm{mg} / \mathrm{L}$ & $2.0 \mathrm{mg} / \mathrm{L}$ & $3.0 \mathrm{mg} / \mathrm{L}$ & $4.0 \mathrm{mg} / \mathrm{L}$ & $5.0 \mathrm{mg} / \mathrm{L}$ \\
\hline 250 & 10.0000 & 1.1642 & 0.7094 & 0.5758 & 0.5172 & 0.4846 & 0.5375 & 0.5774 & 0.6468 \\
\hline 249 & 10.0000 & 1.1705 & 0.7150 & 0.5814 & 0.5229 & 0.4901 & 0.5430 & 0.5830 & 0.6528 \\
\hline 248 & 10.0000 & 1.1767 & 0.7211 & 0.5871 & 0.5285 & 0.4956 & 0.5485 & 0.5887 & 0.6585 \\
\hline 247 & 10.0000 & 1.1826 & 0.7267 & 0.5927 & 0.5341 & 0.5011 & 0.5540 & 0.5944 & 0.6645 \\
\hline 246 & 10.0000 & 1.1887 & 0.7324 & 0.5982 & 0.5395 & 0.5065 & 0.5594 & 0.5996 & 0.6702 \\
\hline 245 & 10.0000 & 1.1943 & 0.7380 & 0.6035 & 0.5445 & 0.5114 & 0.5646 & 0.6049 & 0.6755 \\
\hline 244 & 10.0000 & 1.1999 & 0.7431 & 0.6085 & 0.5496 & 0.5163 & 0.5695 & 0.6099 & 0.6807 \\
\hline 243 & 10.0000 & 1.2052 & 0.7481 & 0.6130 & 0.5543 & 0.5209 & 0.5741 & 0.6147 & 0.6856 \\
\hline 242 & 10.0000 & 1.2102 & 0.7526 & 0.6176 & 0.5586 & 0.5252 & 0.5781 & 0.6190 & 0.6901 \\
\hline 241 & 10.0000 & 1.2148 & 0.7571 & 0.6219 & 0.5627 & 0.5293 & 0.5823 & 0.6230 & 0.6945 \\
\hline 240 & 10.0000 & 1.2194 & 0.7611 & 0.6258 & 0.5666 & 0.5330 & 0.5862 & 0.6270 & 0.6987 \\
\hline 239 & 10.0000 & 1.2237 & 0.7654 & 0.6297 & 0.5702 & 0.5365 & 0.5898 & 0.6308 & 0.7025 \\
\hline 238 & 10.0000 & 1.2277 & 0.7693 & 0.6332 & 0.5738 & 0.5400 & 0.5934 & 0.6343 & 0.7064 \\
\hline 237 & 10.0000 & 1.2320 & 0.7733 & 0.6370 & 0.5774 & 0.5434 & 0.5970 & 0.6380 & 0.7102 \\
\hline 236 & 10.0000 & 1.2365 & 0.7772 & 0.6409 & 0.5811 & 0.5470 & 0.6004 & 0.6415 & 0.7141 \\
\hline 235 & 10.0000 & 1.2411 & 0.7814 & 0.6447 & 0.5849 & 0.5506 & 0.6043 & 0.6456 & 0.7183 \\
\hline 234 & 10.0000 & 1.2460 & 0.7859 & 0.6491 & 0.5890 & 0.5545 & 0.6084 & 0.6498 & 0.7230 \\
\hline 233 & 10.0000 & 1.2516 & 0.7911 & 0.6538 & 0.5936 & 0.5591 & 0.6129 & 0.6544 & 0.7279 \\
\hline 232 & 10.0000 & 1.2575 & 0.7966 & 0.6592 & 0.5988 & 0.5640 & 0.6180 & 0.6596 & 0.7333 \\
\hline 231 & 10.0000 & 1.2640 & 0.8029 & 0.6650 & 0.6046 & 0.5698 & 0.6238 & 0.6654 & 0.7396 \\
\hline 230 & 10.0000 & 1.2715 & 0.8097 & 0.6714 & 0.6110 & 0.5760 & 0.6303 & 0.6718 & 0.7467 \\
\hline 229 & 10.0000 & 1.2796 & 0.8174 & 0.6789 & 0.6185 & 0.5832 & 0.6375 & 0.6793 & 0.7542 \\
\hline 228 & 10.0000 & 1.2887 & 0.8261 & 0.6874 & 0.6267 & 0.5913 & 0.6458 & 0.6876 & 0.7629 \\
\hline 227 & 10.0000 & 1.2990 & 0.8358 & 0.6968 & 0.6358 & 0.6003 & 0.6549 & 0.6969 & 0.7725 \\
\hline 226 & 10.0000 & 1.3106 & 0.8466 & 0.7072 & 0.6461 & 0.6104 & 0.6651 & 0.7072 & 0.7832 \\
\hline 225 & 10.0000 & 1.3229 & 0.8586 & 0.7186 & 0.6576 & 0.6218 & 0.6768 & 0.7187 & 0.7952 \\
\hline 224 & 10.0000 & 1.3365 & 0.8716 & 0.7317 & 0.6703 & 0.6344 & 0.6893 & 0.7315 & 0.8082 \\
\hline 223 & 10.0000 & 1.3516 & 0.8860 & 0.7456 & 0.6843 & 0.6481 & 0.7033 & 0.7454 & 0.8228 \\
\hline 222 & 10.0000 & 1.3680 & 0.9017 & 0.7613 & 0.6997 & 0.6630 & 0.7184 & 0.7606 & 0.8384 \\
\hline 221 & 10.0000 & 1.3858 & 0.9189 & 0.7781 & 0.7162 & 0.6797 & 0.7349 & 0.7774 & 0.8554 \\
\hline 220 & 10.0000 & 1.4048 & 0.9372 & 0.7960 & 0.7340 & 0.6973 & 0.7529 & 0.7954 & 0.8739 \\
\hline 219 & 10.0000 & 1.4250 & 0.9566 & 0.8153 & 0.7531 & 0.7159 & 0.7719 & 0.8145 & 0.8932 \\
\hline
\end{tabular}




\begin{tabular}{|c|c|c|c|c|c|c|c|c|c|}
\hline 218 & 10.0000 & 1.4463 & 0.9771 & 0.8354 & 0.7733 & 0.7358 & 0.7922 & 0.8346 & 0.9138 \\
\hline 217 & 10.0000 & 1.4682 & 0.9986 & 0.8566 & 0.7940 & 0.7567 & 0.8131 & 0.8557 & 0.9351 \\
\hline 216 & 10.0000 & 1.4907 & 1.0205 & 0.8782 & 0.8156 & 0.7779 & 0.8346 & 0.8773 & 0.9573 \\
\hline 215 & 10.0000 & 1.5144 & 1.0430 & 0.9004 & 0.8377 & 0.7999 & 0.8568 & 0.8996 & 0.9798 \\
\hline 214 & 10.0000 & 1.5377 & 1.0660 & 0.9232 & 0.8604 & 0.8222 & 0.8795 & 0.9220 & 1.0029 \\
\hline 213 & 10.0000 & 1.5612 & 1.0893 & 0.9461 & 0.8832 & 0.8449 & 0.9023 & 0.9452 & 1.0262 \\
\hline 212 & 10.0000 & 1.5851 & 1.1125 & 0.9694 & 0.9060 & 0.8675 & 0.9254 & 0.9679 & 1.0499 \\
\hline 211 & 10.0000 & 1.6091 & 1.1357 & 0.9921 & 0.9289 & 0.8901 & 0.9483 & 0.9909 & 1.0734 \\
\hline 210 & 10.0000 & 1.6326 & 1.1585 & 1.0148 & 0.9515 & 0.9124 & 0.9708 & 1.0134 & 1.0964 \\
\hline 209 & 10.0000 & 1.6561 & 1.1810 & 1.0373 & 0.9736 & 0.9344 & 0.9934 & 1.0360 & 1.1193 \\
\hline 208 & 10.0000 & 1.6790 & 1.2031 & 1.0592 & 0.9958 & 0.9558 & 1.0154 & 1.0580 & 1.1419 \\
\hline 207 & 10.0000 & 1.7016 & 1.2249 & 1.0810 & 1.0174 & 0.9773 & 1.0373 & 1.0801 & 1.1644 \\
\hline 206 & 10.0000 & 1.7246 & 1.2472 & 1.1031 & 1.0395 & 0.9991 & 1.0596 & 1.1024 & 1.1873 \\
\hline 205 & 10.0000 & 1.7469 & 1.2694 & 1.1252 & 1.0616 & 1.0209 & 1.0823 & 1.1251 & 1.2108 \\
\hline 204 & 10.0000 & 1.7707 & 1.2921 & 1.1483 & 1.0849 & 1.0439 & 1.1058 & 1.1490 & 1.2359 \\
\hline 203 & 10.0000 & 1.7955 & 1.3166 & 1.1723 & 1.1097 & 1.0683 & 1.1311 & 1.1747 & 1.2628 \\
\hline 202 & 10.0000 & 1.8221 & 1.3432 & 1.1991 & 1.1367 & 1.0949 & 1.1590 & 1.2032 & 1.2923 \\
\hline 201 & 10.0000 & 1.8519 & 1.3733 & 1.2295 & 1.1674 & 1.1255 & 1.1907 & 1.2360 & 1.3270 \\
\hline 200 & 10.0000 & 1.8867 & 1.4074 & 1.2642 & 1.2026 & 1.1605 & 1.2275 & 1.2738 & 1.3669 \\
\hline 199 & 10.0000 & 1.9267 & 1.4473 & 1.3044 & 1.2433 & 1.2017 & 1.2700 & 1.3176 & 1.4130 \\
\hline 198 & 10.0000 & 1.9756 & 1.4956 & 1.3533 & 1.2931 & 1.2512 & 1.3218 & 1.3703 & 1.4683 \\
\hline 197 & 10.0000 & 2.0345 & 1.5560 & 1.4147 & 1.3536 & 1.3119 & 1.3859 & 1.4366 & 1.5368 \\
\hline 196 & 10.0000 & 2.1057 & 1.6220 & 1.4814 & 1.4256 & 1.3838 & 1.4556 & 1.5082 & 1.6105 \\
\hline 195 & 10.0000 & 2.1898 & 1.7093 & 1.5689 & 1.5119 & 1.4694 & 1.5468 & 1.6014 & 1.7059 \\
\hline 194 & 10.0000 & 2.2922 & 1.8121 & 1.6728 & 1.6153 & 1.5731 & 1.6528 & 1.7097 & 1.8158 \\
\hline 193 & 10.0000 & 2.4226 & 1.9404 & 1.8019 & 1.7455 & 1.7030 & 1.7846 & 1.8437 & 1.9514 \\
\hline 192 & 10.0000 & 2.5979 & 2.1143 & 1.9761 & 1.9188 & 1.8772 & 1.9607 & 2.0221 & 2.1300 \\
\hline 191 & 10.0000 & 2.7679 & 2.2827 & 2.1470 & 2.0910 & 2.0488 & 2.1320 & 2.1956 & 2.3024 \\
\hline 190 & 10.0000 & 2.9400 & 2.4575 & 2.3220 & 2.2645 & 2.2220 & 2.3049 & 2.3675 & 2.4706 \\
\hline
\end{tabular}




\begin{tabular}{|c|c|c|c|c|c|c|c|c|c|}
\hline \multicolumn{10}{|c|}{ Magnafloc determination of optimum dose in high turbidity Rideau River water (9172 NTU) } \\
\hline \multicolumn{10}{|c|}{ In-line spectrophotometer (4mm path length) } \\
\hline Wavelength (nm) & $0 \mathrm{mg} / \mathrm{L}$ & $0.1 \mathrm{mg} / \mathrm{L}$ & $0.5 \mathrm{mg} / \mathrm{L}$ & $1.0 \mathrm{mg} / \mathrm{L}$ & $1.5 \mathrm{mg} / \mathrm{L}$ & $2.0 \mathrm{mg} / \mathrm{L}$ & $3.0 \mathrm{mg} / \mathrm{L}$ & $4.0 \mathrm{mg} / \mathrm{L}$ & $5.0 \mathrm{mg} / \mathrm{L}$ \\
\hline 189.8 & 3.75 & 1.3422 & 1.3376 & 1.3296 & 1.2400 & 1.2364 & 1.3480 & 1.4491 & 1.5923 \\
\hline 192.8 & 3.75 & 1.1556 & 1.1411 & 1.1278 & 1.0451 & 1.0380 & 1.1349 & 1.2153 & 1.3343 \\
\hline 195.8 & 3.75 & 1.0782 & 1.0575 & 1.0398 & 0.9619 & 0.9548 & 1.0464 & 1.1160 & 1.2249 \\
\hline 198.9 & 3.75 & 1.0084 & 0.9835 & 0.9618 & 0.8888 & 0.8804 & 0.9672 & 1.0328 & 1.1328 \\
\hline 201.9 & 3.75 & 0.9567 & 0.9261 & 0.9009 & 0.8316 & 0.8231 & 0.9066 & 0.9694 & 1.0639 \\
\hline 204.9 & 3.75 & 0.8926 & 0.8593 & 0.8337 & 0.7664 & 0.7580 & 0.8388 & 0.8972 & 0.9885 \\
\hline 208 & 3.75 & 0.8351 & 0.7981 & 0.7688 & 0.7065 & 0.6972 & 0.7741 & 0.8310 & 0.9185 \\
\hline 211 & 3.75 & 0.7844 & 0.7425 & 0.7118 & 0.6506 & 0.6422 & 0.7177 & 0.7721 & 0.8576 \\
\hline 214 & 3.75 & 0.7413 & 0.6954 & 0.6622 & 0.6048 & 0.5954 & 0.6705 & 0.7223 & 0.8046 \\
\hline 217 & 3.75 & 0.6986 & 0.6506 & 0.6153 & 0.5606 & 0.5501 & 0.6237 & 0.6745 & 0.7555 \\
\hline 220 & 3.75 & 0.6741 & 0.6208 & 0.5853 & 0.5322 & 0.5220 & 0.5949 & 0.6457 & 0.7240 \\
\hline 223.1 & 3.75 & 0.6457 & 0.5899 & 0.5540 & 0.5034 & 0.4926 & 0.5640 & 0.6127 & 0.6912 \\
\hline 226.1 & 3.75 & 0.6273 & 0.5687 & 0.5321 & 0.4818 & 0.4715 & 0.5414 & 0.5898 & 0.6671 \\
\hline 229.1 & 3.75 & 0.6096 & 0.5502 & 0.5126 & 0.4644 & 0.4530 & 0.5222 & 0.5697 & 0.6468 \\
\hline 232.1 & 3.75 & 0.5985 & 0.5363 & 0.4988 & 0.4526 & 0.4402 & 0.5084 & 0.5547 & 0.6308 \\
\hline 235.1 & 3.75 & 0.5902 & 0.5267 & 0.4885 & 0.4420 & 0.4296 & 0.4980 & 0.5433 & 0.6195 \\
\hline 238.1 & 3.75 & 0.5831 & 0.5180 & 0.4801 & 0.4345 & 0.4218 & 0.4888 & 0.5359 & 0.6113 \\
\hline 241.1 & 3.75 & 0.5795 & 0.5120 & 0.4729 & 0.4282 & 0.4156 & 0.4822 & 0.5278 & 0.6048 \\
\hline 244.1 & 3.75 & 0.5766 & 0.5069 & 0.4675 & 0.4232 & 0.4096 & 0.4766 & 0.5222 & 0.5984 \\
\hline 247.1 & 3.75 & 0.5707 & 0.5029 & 0.4633 & 0.4195 & 0.4052 & 0.4719 & 0.5170 & 0.5932 \\
\hline 250.1 & 3.75 & 0.5683 & 0.4993 & 0.4585 & 0.4149 & 0.4010 & 0.4672 & 0.5134 & 0.5886 \\
\hline
\end{tabular}




\begin{tabular}{|c|c|c|c|c|c|c|c|c|}
\hline \multicolumn{9}{|c|}{ Magnafloc determination of optimum dose in low turbidity Rideau River water (260 NTU) } \\
\hline \multicolumn{9}{|c|}{ Desktop spectrophotometer (1 cm path length) } \\
\hline $\begin{array}{l}\text { Wavelength } \\
(\mathrm{nm})\end{array}$ & $0 \mathrm{mg} / \mathrm{L}$ & $0.05 \mathrm{mg} / \mathrm{L}$ & $0.1 \mathrm{mg} / \mathrm{L}$ & $0.5 \mathrm{mg} / \mathrm{L}$ & $1.0 \mathrm{mg} / \mathrm{L}$ & $1.5 \mathrm{mg} / \mathrm{L}$ & $2.0 \mathrm{mg} / \mathrm{L}$ & $3.0 \mathrm{mg} / \mathrm{L}$ \\
\hline 250 & 0.5943 & 0.4553 & 0.3972 & 0.4065 & 0.4084 & 0.4135 & 0.4531 & 0.4298 \\
\hline 249 & 0.5993 & 0.4606 & 0.4026 & 0.4117 & 0.4138 & 0.4188 & 0.4586 & 0.4351 \\
\hline 248 & 0.6048 & 0.4658 & 0.4081 & 0.4171 & 0.4191 & 0.4243 & 0.4639 & 0.4407 \\
\hline 247 & 0.6101 & 0.4712 & 0.4135 & 0.4226 & 0.4246 & 0.4298 & 0.4696 & 0.4461 \\
\hline 246 & 0.6153 & 0.4765 & 0.4188 & 0.4280 & 0.4301 & 0.4352 & 0.4749 & 0.4517 \\
\hline 245 & 0.6204 & 0.4818 & 0.4242 & 0.4333 & 0.4353 & 0.4405 & 0.4804 & 0.4568 \\
\hline 244 & 0.6253 & 0.4867 & 0.4290 & 0.4384 & 0.4404 & 0.4456 & 0.4851 & 0.4619 \\
\hline 243 & 0.6299 & 0.4914 & 0.4339 & 0.4432 & 0.4450 & 0.4504 & 0.4900 & 0.4668 \\
\hline 242 & 0.6342 & 0.4958 & 0.4383 & 0.4476 & 0.4495 & 0.4549 & 0.4944 & 0.4712 \\
\hline 241 & 0.6381 & 0.4998 & 0.4424 & 0.4519 & 0.4537 & 0.4591 & 0.4987 & 0.4755 \\
\hline 240 & 0.6419 & 0.5037 & 0.4463 & 0.4558 & 0.4576 & 0.4630 & 0.5026 & 0.4795 \\
\hline 239 & 0.6454 & 0.5074 & 0.4498 & 0.4595 & 0.4612 & 0.4668 & 0.5064 & 0.4834 \\
\hline 238 & 0.6487 & 0.5106 & 0.4532 & 0.4632 & 0.4647 & 0.4703 & 0.5099 & 0.4869 \\
\hline 237 & 0.6518 & 0.5138 & 0.4565 & 0.4666 & 0.4680 & 0.4737 & 0.5134 & 0.4904 \\
\hline 236 & 0.6548 & 0.5170 & 0.4596 & 0.4697 & 0.4710 & 0.4767 & 0.5166 & 0.4935 \\
\hline 235 & 0.6579 & 0.5201 & 0.4628 & 0.4729 & 0.4743 & 0.4802 & 0.5198 & 0.4967 \\
\hline 234 & 0.6609 & 0.5233 & 0.4660 & 0.4762 & 0.4777 & 0.4834 & 0.5231 & 0.5000 \\
\hline 233 & 0.6642 & 0.5268 & 0.4694 & 0.4796 & 0.4811 & 0.4871 & 0.5267 & 0.5037 \\
\hline 232 & 0.6678 & 0.5304 & 0.4731 & 0.4833 & 0.4849 & 0.4907 & 0.5304 & 0.5075 \\
\hline 231 & 0.6716 & 0.5344 & 0.4770 & 0.4873 & 0.4889 & 0.4949 & 0.5346 & 0.5117 \\
\hline 230 & 0.6758 & 0.5386 & 0.4813 & 0.4916 & 0.4933 & 0.4992 & 0.5391 & 0.5162 \\
\hline 229 & 0.6802 & 0.5434 & 0.4860 & 0.4964 & 0.4980 & 0.5042 & 0.5440 & 0.5211 \\
\hline 228 & 0.6853 & 0.5486 & 0.4910 & 0.5015 & 0.5033 & 0.5093 & 0.5492 & 0.5264 \\
\hline 227 & 0.6908 & 0.5541 & 0.4968 & 0.5072 & 0.5091 & 0.5152 & 0.5551 & 0.5323 \\
\hline 226 & 0.6969 & 0.5605 & 0.5030 & 0.5133 & 0.5153 & 0.5216 & 0.5615 & 0.5389 \\
\hline 225 & 0.7036 & 0.5674 & 0.5099 & 0.5202 & 0.5224 & 0.5287 & 0.5687 & 0.5462 \\
\hline 224 & 0.7109 & 0.5749 & 0.5174 & 0.5277 & 0.5301 & 0.5366 & 0.5764 & 0.5539 \\
\hline 223 & 0.7192 & 0.5833 & 0.5258 & 0.5362 & 0.5384 & 0.5452 & 0.5851 & 0.5626 \\
\hline 222 & 0.7280 & 0.5924 & 0.5349 & 0.5454 & 0.5477 & 0.5546 & 0.5945 & 0.5723 \\
\hline 221 & 0.7378 & 0.6026 & 0.5450 & 0.5554 & 0.5581 & 0.5649 & 0.6050 & 0.5826 \\
\hline 220 & 0.7483 & 0.6135 & 0.5560 & 0.5664 & 0.5690 & 0.5762 & 0.6163 & 0.5943 \\
\hline 219 & 0.7599 & 0.6253 & 0.5677 & 0.5784 & 0.5810 & 0.5883 & 0.6286 & 0.6066 \\
\hline 218 & 0.7724 & 0.6380 & 0.5805 & 0.5911 & 0.5939 & 0.6013 & 0.6417 & 0.6200 \\
\hline 217 & 0.7854 & 0.6514 & 0.5939 & 0.6048 & 0.6076 & 0.6154 & 0.6558 & 0.6343 \\
\hline 216 & 0.7992 & 0.6654 & 0.6080 & 0.6190 & 0.6220 & 0.6299 & 0.6705 & 0.6492 \\
\hline
\end{tabular}




\begin{tabular}{|c|c|c|c|c|c|c|c|c|}
\hline 215 & 0.8136 & 0.6802 & 0.6228 & 0.6340 & 0.6370 & 0.6451 & 0.6858 & 0.6648 \\
\hline 214 & 0.8287 & 0.6955 & 0.6383 & 0.6498 & 0.6528 & 0.6611 & 0.7020 & 0.6811 \\
\hline 213 & 0.8443 & 0.7115 & 0.6542 & 0.6662 & 0.6692 & 0.6778 & 0.7187 & 0.6983 \\
\hline 212 & 0.8604 & 0.7282 & 0.6710 & 0.6832 & 0.6862 & 0.6950 & 0.7362 & 0.7159 \\
\hline 211 & 0.8772 & 0.7452 & 0.6880 & 0.7009 & 0.7035 & 0.7130 & 0.7544 & 0.7343 \\
\hline 210 & 0.8944 & 0.7627 & 0.7057 & 0.7191 & 0.7214 & 0.7311 & 0.7725 & 0.7530 \\
\hline 209 & 0.9121 & 0.7808 & 0.7239 & 0.7379 & 0.7399 & 0.7500 & 0.7918 & 0.7724 \\
\hline 208 & 0.9299 & 0.7991 & 0.7423 & 0.7570 & 0.7589 & 0.7689 & 0.8113 & 0.7921 \\
\hline 207 & 0.9486 & 0.8181 & 0.7614 & 0.7769 & 0.7783 & 0.7890 & 0.8314 & 0.8126 \\
\hline 206 & 0.9681 & 0.8380 & 0.7811 & 0.7976 & 0.7988 & 0.8096 & 0.8526 & 0.8341 \\
\hline 205 & 0.9882 & 0.8588 & 0.8021 & 0.8194 & 0.8207 & 0.8319 & 0.8746 & 0.8569 \\
\hline 204 & 1.0097 & 0.8808 & 0.8242 & 0.8425 & 0.8436 & 0.8554 & 0.8984 & 0.8813 \\
\hline 203 & 1.0335 & 0.9053 & 0.8485 & 0.8675 & 0.8688 & 0.8811 & 0.9245 & 0.9085 \\
\hline 202 & 1.0597 & 0.9321 & 0.8756 & 0.8954 & 0.8973 & 0.9100 & 0.9540 & 0.9389 \\
\hline 201 & 1.0899 & 0.9634 & 0.9068 & 0.9272 & 0.9300 & 0.9432 & 0.9878 & 0.9741 \\
\hline 200 & 1.1253 & 0.9998 & 0.9434 & 0.9641 & 0.9681 & 0.9821 & 1.0275 & 1.0151 \\
\hline 199 & 1.1677 & 1.0437 & 0.9868 & 1.0082 & 1.0138 & 1.0282 & 1.0742 & 1.0637 \\
\hline 198 & 1.2185 & 1.0953 & 1.0386 & 1.0607 & 1.0677 & 1.0828 & 1.1297 & 1.1216 \\
\hline 197 & 1.2826 & 1.1605 & 1.1043 & 1.1253 & 1.1349 & 1.1505 & 1.1999 & 1.1940 \\
\hline 196 & 1.3587 & 1.2421 & 1.1822 & 1.2069 & 1.2183 & 1.2347 & 1.2822 & 1.2790 \\
\hline 195 & 1.4510 & 1.3347 & 1.2765 & 1.2997 & 1.3140 & 1.3296 & 1.3811 & 1.3801 \\
\hline 194 & 1.5693 & 1.4568 & 1.3978 & 1.4213 & 1.4386 & 1.4542 & 1.5063 & 1.5084 \\
\hline 193 & 1.7116 & 1.6022 & 1.5426 & 1.5667 & 1.5863 & 1.6015 & 1.6551 & 1.6602 \\
\hline 192 & 1.8881 & 1.7827 & 1.7222 & 1.7462 & 1.7685 & 1.7819 & 1.8375 & 1.8437 \\
\hline 191 & 2.1244 & 2.0228 & 1.9615 & 1.9858 & 2.0099 & 2.0208 & 2.0779 & 2.0837 \\
\hline 190 & 2.3323 & 2.2312 & 2.1700 & 2.1923 & 2.2152 & 2.2256 & 2.2794 & 2.2847 \\
\hline
\end{tabular}




\begin{tabular}{|c|c|c|c|c|c|c|c|c|}
\hline \multicolumn{9}{|c|}{ Magnafloc determination of optimum dose in low turbidity Rideau River water (260 NTU) } \\
\hline \multicolumn{9}{|c|}{ In-line spectrophotometer (4mm path length) } \\
\hline $\begin{array}{l}\text { Wavelength } \\
(\mathrm{nm})\end{array}$ & $0 \mathrm{mg} / \mathrm{L}$ & $0.05 \mathrm{mg} / \mathrm{L}$ & $0.1 \mathrm{mg} / \mathrm{L}$ & $0.5 \mathrm{mg} / \mathrm{L}$ & $1.0 \mathrm{mg} / \mathrm{L}$ & $1.5 \mathrm{mg} / \mathrm{L}$ & $2.0 \mathrm{mg} / \mathrm{L}$ & $3.0 \mathrm{mg} / \mathrm{L}$ \\
\hline 189.8 & 1.3154 & 1.1800 & 1.0548 & 1.1990 & 1.1453 & 1.1588 & 1.2220 & 1.2211 \\
\hline 192.8 & 1.0748 & 0.9507 & 0.8489 & 0.9651 & 0.9226 & 0.9300 & 0.9851 & 0.9765 \\
\hline 195.8 & 0.9792 & 0.8562 & 0.7669 & 0.8703 & 0.8317 & 0.8387 & 0.8885 & 0.8794 \\
\hline 198.9 & 0.9068 & 0.7810 & 0.7009 & 0.7918 & 0.7600 & 0.7667 & 0.8102 & 0.8004 \\
\hline 201.9 & 0.8546 & 0.7296 & 0.6550 & 0.7377 & 0.7106 & 0.7160 & 0.7573 & 0.7494 \\
\hline 204.9 & 0.8043 & 0.6810 & 0.6093 & 0.6865 & 0.6626 & 0.6673 & 0.7078 & 0.6990 \\
\hline 208 & 0.7623 & 0.6399 & 0.5710 & 0.6441 & 0.6223 & 0.6270 & 0.6671 & 0.6579 \\
\hline 211 & 0.7266 & 0.6026 & 0.5393 & 0.6072 & 0.5881 & 0.5930 & 0.6340 & 0.6223 \\
\hline 214 & 0.6972 & 0.5699 & 0.5114 & 0.5762 & 0.5586 & 0.5641 & 0.6023 & 0.5919 \\
\hline 217 & 0.6654 & 0.5392 & 0.4821 & 0.5441 & 0.5281 & 0.5336 & 0.5705 & 0.5609 \\
\hline 220 & 0.6442 & 0.5178 & 0.4621 & 0.5213 & 0.5074 & 0.5120 & 0.5482 & 0.5389 \\
\hline 223.1 & 0.6192 & 0.4939 & 0.4396 & 0.4969 & 0.4844 & 0.4883 & 0.5246 & 0.5157 \\
\hline 226.1 & 0.6023 & 0.4752 & 0.4233 & 0.4794 & 0.4671 & 0.4711 & 0.5074 & 0.4983 \\
\hline 229.1 & 0.5858 & 0.4556 & 0.4061 & 0.4606 & 0.4482 & 0.4533 & 0.4895 & 0.4797 \\
\hline 232.1 & 0.5714 & 0.4407 & 0.3928 & 0.4451 & 0.4333 & 0.4387 & 0.4746 & 0.4648 \\
\hline 235.1 & 0.5604 & 0.4284 & 0.3818 & 0.4316 & 0.4215 & 0.4265 & 0.4612 & 0.4520 \\
\hline 238.1 & 0.5501 & 0.4192 & 0.3721 & 0.4209 & 0.4118 & 0.4162 & 0.4510 & 0.4427 \\
\hline 241.1 & 0.5440 & 0.4106 & 0.3637 & 0.4119 & 0.4033 & 0.4075 & 0.4422 & 0.4341 \\
\hline 244.1 & 0.5407 & 0.4045 & 0.3586 & 0.4066 & 0.3976 & 0.4023 & 0.4382 & 0.4286 \\
\hline 247.1 & 0.5350 & 0.3974 & 0.3518 & 0.3984 & 0.3900 & 0.3963 & 0.4314 & 0.4225 \\
\hline 250.1 & 0.5301 & 0.3922 & 0.3463 & 0.3925 & 0.3840 & 0.3901 & 0.4245 & 0.4163 \\
\hline
\end{tabular}




\begin{tabular}{|c|c|c|c|c|c|c|c|c|}
\hline \multicolumn{9}{|c|}{ Desktop spectrophotometer (1 cm path length) } \\
\hline $\begin{array}{l}\text { Wavelength } \\
(\mathrm{nm})\end{array}$ & $0.05 \mathrm{mg} / \mathrm{L}$ & $0.1 \mathrm{mg} / \mathrm{L}$ & $0.5 \mathrm{mg} / \mathrm{L}$ & $1.0 \mathrm{mg} / \mathrm{L}$ & $2.0 \mathrm{mg} / \mathrm{L}$ & $4.0 \mathrm{mg} / \mathrm{L}$ & $6.0 \mathrm{mg} / \mathrm{L}$ & $8.0 \mathrm{mg} / \mathrm{L}$ \\
\hline 250 & 2.0896 & 1.5218 & 0.9666 & 0.9870 & 1.0274 & 1.1188 & 1.2840 & 1.2828 \\
\hline 249 & 2.0952 & 1.5275 & 0.9722 & 0.9926 & 1.0331 & 1.1244 & 1.2901 & 1.2890 \\
\hline 248 & 2.1015 & 1.5331 & 0.9779 & 0.9985 & 1.0390 & 1.1303 & 1.2966 & 1.2954 \\
\hline 247 & 2.1076 & 1.5391 & 0.9837 & 1.0043 & 1.0448 & 1.1363 & 1.3029 & 1.3019 \\
\hline 246 & 2.1126 & 1.5449 & 0.9892 & 1.0101 & 1.0504 & 1.1421 & 1.3093 & 1.3082 \\
\hline 245 & 2.1184 & 1.5501 & 0.9948 & 1.0155 & 1.0562 & 1.1477 & 1.3154 & 1.3144 \\
\hline 244 & 2.1233 & 1.5552 & 1.0001 & 1.0207 & 1.0613 & 1.1530 & 1.3212 & 1.3202 \\
\hline 243 & 2.1288 & 1.5603 & 1.0051 & 1.0259 & 1.0665 & 1.1583 & 1.3272 & 1.3260 \\
\hline 242 & 2.1334 & 1.5648 & 1.0096 & 1.0306 & 1.0712 & 1.1630 & 1.3325 & 1.3316 \\
\hline 241 & 2.1376 & 1.5688 & 1.0140 & 1.0351 & 1.0755 & 1.1676 & 1.3376 & 1.3366 \\
\hline 240 & 2.1420 & 1.5730 & 1.0183 & 1.0393 & 1.0798 & 1.1721 & 1.3426 & 1.3417 \\
\hline 239 & 2.1461 & 1.5766 & 1.0219 & 1.0432 & 1.0838 & 1.1760 & 1.3476 & 1.3464 \\
\hline 238 & 2.1490 & 1.5803 & 1.0256 & 1.0470 & 1.0876 & 1.1798 & 1.3520 & 1.3508 \\
\hline 237 & 2.1534 & 1.5837 & 1.0290 & 1.0505 & 1.0911 & 1.1835 & 1.3565 & 1.3552 \\
\hline 236 & 2.1570 & 1.5871 & 1.0323 & 1.0541 & 1.0945 & 1.1871 & 1.3607 & 1.3598 \\
\hline 235 & 2.1605 & 1.5905 & 1.0355 & 1.0573 & 1.0980 & 1.1904 & 1.3651 & 1.3640 \\
\hline 234 & 2.1639 & 1.5938 & 1.0388 & 1.0608 & 1.1013 & 1.1941 & 1.3695 & 1.3684 \\
\hline 233 & 2.1677 & 1.5973 & 1.0420 & 1.0644 & 1.1048 & 1.1980 & 1.3740 & 1.3731 \\
\hline 232 & 2.1722 & 1.6007 & 1.0456 & 1.0680 & 1.1085 & 1.2018 & 1.3787 & 1.3778 \\
\hline 231 & 2.1766 & 1.6047 & 1.0494 & 1.0719 & 1.1124 & 1.2057 & 1.3839 & 1.3828 \\
\hline 230 & 2.1812 & 1.6086 & 1.0534 & 1.0760 & 1.1167 & 1.2101 & 1.3890 & 1.3883 \\
\hline 229 & 2.1859 & 1.6130 & 1.0575 & 1.0804 & 1.1210 & 1.2149 & 1.3948 & 1.3939 \\
\hline 228 & 2.1917 & 1.6185 & 1.0620 & 1.0853 & 1.1259 & 1.2196 & 1.4010 & 1.4001 \\
\hline 227 & 2.1971 & 1.6243 & 1.0671 & 1.0903 & 1.1312 & 1.2252 & 1.4073 & 1.4065 \\
\hline 226 & 2.2029 & 1.6294 & 1.0722 & 1.0960 & 1.1367 & 1.2310 & 1.4142 & 1.4135 \\
\hline 225 & 2.2101 & 1.6359 & 1.0780 & 1.1022 & 1.1429 & 1.2372 & 1.4217 & 1.4212 \\
\hline 224 & 2.2171 & 1.6428 & 1.0842 & 1.1086 & 1.1496 & 1.2442 & 1.4296 & 1.4293 \\
\hline 223 & 2.2256 & 1.6506 & 1.0913 & 1.1160 & 1.1568 & 1.2518 & 1.4386 & 1.4383 \\
\hline 222 & 2.2339 & 1.6585 & 1.0990 & 1.1240 & 1.1648 & 1.2600 & 1.4480 & 1.4476 \\
\hline 221 & 2.2433 & 1.6677 & 1.1073 & 1.1329 & 1.1736 & 1.2693 & 1.4583 & 1.4583 \\
\hline 220 & 2.2532 & 1.6776 & 1.1164 & 1.1423 & 1.1830 & 1.2788 & 1.4693 & 1.4697 \\
\hline 219 & 2.2643 & 1.6882 & 1.1264 & 1.1525 & 1.1934 & 1.2897 & 1.4817 & 1.4819 \\
\hline 218 & 2.2783 & 1.6993 & 1.1369 & 1.1637 & 1.2044 & 1.3024 & 1.4944 & 1.4947 \\
\hline 217 & 2.2910 & 1.7120 & 1.1486 & 1.1756 & 1.2164 & 1.3153 & 1.5081 & 1.5090 \\
\hline 216 & 2.3034 & 1.7245 & 1.1605 & 1.1879 & 1.2287 & 1.3283 & 1.5224 & 1.5234 \\
\hline
\end{tabular}




\begin{tabular}{|c|c|c|c|c|c|c|c|c|}
\hline 215 & 2.3181 & 1.7382 & 1.1736 & 1.2013 & 1.2422 & 1.3421 & 1.5377 & 1.5390 \\
\hline 214 & 2.3330 & 1.7529 & 1.1868 & 1.2151 & 1.2561 & 1.3567 & 1.5543 & 1.5552 \\
\hline 213 & 2.3487 & 1.7680 & 1.2018 & 1.2299 & 1.2721 & 1.3722 & 1.5730 & 1.5750 \\
\hline 212 & 2.3646 & 1.7843 & 1.2165 & 1.2452 & 1.2883 & 1.3880 & 1.5906 & 1.5931 \\
\hline 211 & 2.3833 & 1.8015 & 1.2327 & 1.2633 & 1.3046 & 1.4051 & 1.6095 & 1.6121 \\
\hline 210 & 2.4001 & 1.8190 & 1.2509 & 1.2804 & 1.3220 & 1.4225 & 1.6286 & 1.6317 \\
\hline 209 & 2.4186 & 1.8378 & 1.2691 & 1.2989 & 1.3399 & 1.4412 & 1.6490 & 1.6528 \\
\hline 208 & 2.4382 & 1.8585 & 1.2875 & 1.3173 & 1.3585 & 1.4600 & 1.6704 & 1.6742 \\
\hline 207 & 2.4574 & 1.8800 & 1.3072 & 1.3370 & 1.3782 & 1.4800 & 1.6926 & 1.6969 \\
\hline 206 & 2.4785 & 1.9023 & 1.3279 & 1.3573 & 1.3985 & 1.5031 & 1.7160 & 1.7212 \\
\hline 205 & 2.5001 & 1.9264 & 1.3505 & 1.3800 & 1.4211 & 1.5268 & 1.7419 & 1.7478 \\
\hline 204 & 2.5246 & 1.9524 & 1.3746 & 1.4034 & 1.4443 & 1.5509 & 1.7689 & 1.7766 \\
\hline 203 & 2.5496 & 1.9807 & 1.4010 & 1.4289 & 1.4717 & 1.5782 & 1.8007 & 1.8113 \\
\hline 202 & 2.5772 & 2.0119 & 1.4312 & 1.4593 & 1.5003 & 1.6080 & 1.8348 & 1.8472 \\
\hline 201 & 2.6080 & 2.0465 & 1.4649 & 1.4914 & 1.5327 & 1.6421 & 1.8725 & 1.8879 \\
\hline 200 & 2.6444 & 2.0878 & 1.5032 & 1.5282 & 1.5692 & 1.6815 & 1.9154 & 1.9350 \\
\hline 199 & 2.6845 & 2.1353 & 1.5469 & 1.5703 & 1.6120 & 1.7276 & 1.9653 & 1.9893 \\
\hline 198 & 2.7319 & 2.1890 & 1.5975 & 1.6192 & 1.6605 & 1.7808 & 2.0239 & 2.0527 \\
\hline 197 & 2.7877 & 2.2522 & 1.6598 & 1.6791 & 1.7218 & 1.8442 & 2.0929 & 2.1276 \\
\hline 196 & 2.8593 & 2.3299 & 1.7332 & 1.7512 & 1.7950 & 1.9205 & 2.1752 & 2.2164 \\
\hline 195 & 2.9295 & 2.4116 & 1.8127 & 1.8286 & 1.8725 & 2.0042 & 2.2616 & 2.3100 \\
\hline 194 & 3.0247 & 2.5165 & 1.9167 & 1.9325 & 1.9776 & 2.1118 & 2.3744 & 2.4277 \\
\hline 193 & 3.1428 & 2.6420 & 2.0388 & 2.0549 & 2.0995 & 2.2388 & 2.5062 & 2.5669 \\
\hline 192 & 3.2896 & 2.7930 & 2.1886 & 2.2017 & 2.2491 & 2.3913 & 2.6589 & 2.7245 \\
\hline 191 & 3.4978 & 2.9924 & 2.3896 & 2.4018 & 2.4496 & 2.5932 & 2.8621 & 2.9317 \\
\hline 190 & 3.6235 & 3.1401 & 2.5410 & 2.5582 & 2.6067 & 2.7454 & 3.0035 & 3.0659 \\
\hline
\end{tabular}




\begin{tabular}{|c|c|c|c|c|c|c|c|c|}
\hline \multicolumn{9}{|c|}{ In-line spectrophotometer (4mm path length) } \\
\hline Wavelength (nm) & $0.05 \mathrm{mg} / \mathrm{L}$ & $0.1 \mathrm{mg} / \mathrm{L}$ & $0.5 \mathrm{mg} / \mathrm{L}$ & $1.0 \mathrm{mg} / \mathrm{L}$ & $2.0 \mathrm{mg} / \mathrm{L}$ & $4.0 \mathrm{mg} / \mathrm{L}$ & $6.0 \mathrm{mg} / \mathrm{L}$ & $8.0 \mathrm{mg} / \mathrm{L}$ \\
\hline 189.8 & 2.3344 & 1.7460 & 1.1214 & 1.3923 & 1.5328 & 1.7201 & 2.0077 & 2.1356 \\
\hline 192.8 & 2.0537 & 1.5049 & 0.9393 & 1.1909 & 1.3167 & 1.4780 & 1.7164 & 1.8226 \\
\hline 195.8 & 1.9408 & 1.4150 & 0.8622 & 1.1087 & 1.2294 & 1.3802 & 1.5989 & 1.6940 \\
\hline 198.9 & 1.8524 & 1.3387 & 0.7986 & 1.0426 & 1.1589 & 1.3017 & 1.5066 & 1.5959 \\
\hline 201.9 & 1.7922 & 1.2865 & 0.7621 & 0.9993 & 1.1135 & 1.2537 & 1.4475 & 1.5363 \\
\hline 204.9 & 1.7430 & 1.2458 & 0.7238 & 0.9590 & 1.0728 & 1.2098 & 1.3951 & 1.4826 \\
\hline 208 & 1.7055 & 1.2066 & 0.6954 & 0.9273 & 1.0393 & 1.1752 & 1.3521 & 1.4398 \\
\hline 211 & 1.6703 & 1.1785 & 0.6695 & 0.8992 & 1.0118 & 1.1449 & 1.3159 & 1.4033 \\
\hline 214 & 1.6455 & 1.1574 & 0.6511 & 0.8770 & 0.9884 & 1.1195 & 1.2866 & 1.3729 \\
\hline 217 & 1.6242 & 1.1393 & 0.6334 & 0.8542 & 0.9648 & 1.0969 & 1.2570 & 1.3444 \\
\hline 220 & 1.6058 & 1.1209 & 0.6179 & 0.8377 & 0.9480 & 1.0780 & 1.2343 & 1.3243 \\
\hline 223.1 & 1.5856 & 1.1043 & 0.6014 & 0.8188 & 0.9279 & 1.0574 & 1.2097 & 1.2966 \\
\hline 226.1 & 1.5678 & 1.0899 & 0.5910 & 0.8037 & 0.9124 & 1.0412 & 1.1907 & 1.2841 \\
\hline 229.1 & 1.5556 & 1.0764 & 0.5787 & 0.7889 & 0.8972 & 1.0252 & 1.1721 & 1.2582 \\
\hline 232.1 & 1.5453 & 1.0689 & 0.5674 & 0.7788 & 0.8846 & 1.0123 & 1.1579 & 1.2511 \\
\hline 235.1 & 1.5363 & 1.0621 & 0.5582 & 0.7689 & 0.8764 & 1.0041 & 1.1481 & 1.2468 \\
\hline 238.1 & 1.5333 & 1.0601 & 0.5532 & 0.7623 & 0.8691 & 0.9977 & 1.1397 & 1.2381 \\
\hline 241.1 & 1.5349 & 1.0554 & 0.5479 & 0.7568 & 0.8645 & 0.9936 & 1.1341 & 1.2325 \\
\hline 244.1 & 1.5387 & 1.0525 & 0.5451 & 0.7541 & 0.8615 & 0.9907 & 1.1315 & 1.2300 \\
\hline 247.1 & 1.5379 & 1.0532 & 0.5442 & 0.7509 & 0.8583 & 0.9888 & 1.1287 & 1.2290 \\
\hline 250.1 & 1.5429 & 1.0523 & 0.5387 & 0.7482 & 0.8576 & 0.9871 & 1.1268 & 1.2281 \\
\hline
\end{tabular}




\begin{tabular}{|c|c|c|c|c|c|c|c|c|}
\hline \multicolumn{9}{|c|}{ Desktop spectrophotometer (1 $\mathrm{cm}$ path length) } \\
\hline $\begin{array}{l}\text { Wavelength } \\
(\mathrm{nm})\end{array}$ & $3.0 \mathrm{mg} / \mathrm{L}$ & $4.0 \mathrm{mg} / \mathrm{L}$ & $5.0 \mathrm{mg} / \mathrm{L}$ & $6.0 \mathrm{mg} / \mathrm{L}$ & $7.0 \mathrm{mg} / \mathrm{L}$ & $8.0 \mathrm{mg} / \mathrm{L}$ & $10.0 \mathrm{mg} / \mathrm{L}$ & $12.0 \mathrm{mg} / \mathrm{L}$ \\
\hline 250 & 0.5035 & 0.3738 & 0.3364 & 0.3109 & 0.3563 & 0.2968 & 0.3305 & 0.3856 \\
\hline 249 & 0.5085 & 0.3787 & 0.3413 & 0.3157 & 0.3612 & 0.3013 & 0.3355 & 0.3909 \\
\hline 248 & 0.5140 & 0.3839 & 0.3466 & 0.3209 & 0.3666 & 0.3062 & 0.3408 & 0.3963 \\
\hline 247 & 0.5194 & 0.3892 & 0.3516 & 0.3255 & 0.3717 & 0.3112 & 0.3460 & 0.4017 \\
\hline 246 & 0.5246 & 0.3941 & 0.3565 & 0.3301 & 0.3766 & 0.3158 & 0.3507 & 0.4069 \\
\hline 245 & 0.5297 & 0.3990 & 0.3612 & 0.3349 & 0.3813 & 0.3206 & 0.3556 & 0.4119 \\
\hline 244 & 0.5343 & 0.4036 & 0.3658 & 0.3393 & 0.3858 & 0.3249 & 0.3601 & 0.4167 \\
\hline 243 & 0.5388 & 0.4076 & 0.3698 & 0.3432 & 0.3899 & 0.3290 & 0.3642 & 0.4211 \\
\hline 242 & 0.5429 & 0.4116 & 0.3735 & 0.3469 & 0.3938 & 0.3325 & 0.3679 & 0.4251 \\
\hline 241 & 0.5466 & 0.4150 & 0.3769 & 0.3502 & 0.3974 & 0.3357 & 0.3713 & 0.4289 \\
\hline 240 & 0.5503 & 0.4182 & 0.3800 & 0.3532 & 0.4004 & 0.3388 & 0.3746 & 0.4325 \\
\hline 239 & 0.5534 & 0.4211 & 0.3828 & 0.3560 & 0.4033 & 0.3415 & 0.3773 & 0.4357 \\
\hline 238 & 0.5564 & 0.4238 & 0.3855 & 0.3586 & 0.4058 & 0.3439 & 0.3800 & 0.4386 \\
\hline 237 & 0.5593 & 0.4262 & 0.3878 & 0.3608 & 0.4083 & 0.3460 & 0.3823 & 0.4413 \\
\hline 236 & 0.5621 & 0.4285 & 0.3901 & 0.3628 & 0.4105 & 0.3481 & 0.3845 & 0.4440 \\
\hline 235 & 0.5647 & 0.4309 & 0.3923 & 0.3651 & 0.4130 & 0.3501 & 0.3868 & 0.4469 \\
\hline 234 & 0.5676 & 0.4333 & 0.3948 & 0.3674 & 0.4153 & 0.3525 & 0.3892 & 0.4497 \\
\hline 233 & 0.5707 & 0.4362 & 0.3974 & 0.3698 & 0.4180 & 0.3548 & 0.3918 & 0.4528 \\
\hline 232 & 0.5743 & 0.4392 & 0.4002 & 0.3726 & 0.4209 & 0.3575 & 0.3946 & 0.4562 \\
\hline 231 & 0.5780 & 0.4425 & 0.4037 & 0.3759 & 0.4244 & 0.3604 & 0.3979 & 0.4599 \\
\hline 230 & 0.5823 & 0.4464 & 0.4075 & 0.3794 & 0.4282 & 0.3639 & 0.4015 & 0.4642 \\
\hline 229 & 0.5871 & 0.4509 & 0.4117 & 0.3838 & 0.4325 & 0.3682 & 0.4058 & 0.4689 \\
\hline 228 & 0.5926 & 0.4559 & 0.4166 & 0.3887 & 0.4374 & 0.3726 & 0.4106 & 0.4742 \\
\hline 227 & 0.5990 & 0.4615 & 0.4224 & 0.3942 & 0.4431 & 0.3780 & 0.4162 & 0.4804 \\
\hline 226 & 0.6057 & 0.4680 & 0.4287 & 0.4002 & 0.4495 & 0.3841 & 0.4223 & 0.4873 \\
\hline 225 & 0.6136 & 0.4753 & 0.4359 & 0.4074 & 0.4567 & 0.3913 & 0.4297 & 0.4951 \\
\hline 224 & 0.6222 & 0.4834 & 0.4438 & 0.4154 & 0.4647 & 0.3991 & 0.4375 & 0.5036 \\
\hline 223 & 0.6319 & 0.4925 & 0.4530 & 0.4241 & 0.4736 & 0.4077 & 0.4465 & 0.5131 \\
\hline 222 & 0.6423 & 0.5026 & 0.4628 & 0.4338 & 0.4835 & 0.4172 & 0.4562 & 0.5235 \\
\hline 221 & 0.6542 & 0.5137 & 0.4739 & 0.4446 & 0.4948 & 0.4282 & 0.4675 & 0.5352 \\
\hline 220 & 0.6665 & 0.5258 & 0.4856 & 0.4563 & 0.5066 & 0.4398 & 0.4791 & 0.5479 \\
\hline 219 & 0.6809 & 0.5391 & 0.4989 & 0.4693 & 0.5199 & 0.4525 & 0.4925 & 0.5618 \\
\hline 218 & 0.6952 & 0.5529 & 0.5125 & 0.4830 & 0.5336 & 0.4658 & 0.5063 & 0.5764 \\
\hline 217 & 0.7109 & 0.5681 & 0.5274 & 0.4975 & 0.5486 & 0.4804 & 0.5212 & 0.5921 \\
\hline 216 & 0.7267 & 0.5835 & 0.5425 & 0.5125 & 0.5638 & 0.4952 & 0.5364 & 0.6086 \\
\hline
\end{tabular}




\begin{tabular}{|c|c|c|c|c|c|c|c|c|}
\hline 215 & 0.7435 & 0.5996 & 0.5587 & 0.5283 & 0.5799 & 0.5108 & 0.5527 & 0.6260 \\
\hline 214 & 0.7604 & 0.6162 & 0.5749 & 0.5445 & 0.5962 & 0.5268 & 0.5689 & 0.6438 \\
\hline 213 & 0.7781 & 0.6336 & 0.5920 & 0.5611 & 0.6132 & 0.5433 & 0.5862 & 0.6627 \\
\hline 212 & 0.7960 & 0.6509 & 0.6089 & 0.5778 & 0.6310 & 0.5599 & 0.6032 & 0.6811 \\
\hline 211 & 0.8140 & 0.6684 & 0.6269 & 0.5951 & 0.6485 & 0.5770 & 0.6214 & 0.7004 \\
\hline 210 & 0.8319 & 0.6857 & 0.6443 & 0.6126 & 0.6658 & 0.5940 & 0.6386 & 0.7191 \\
\hline 209 & 0.8500 & 0.7033 & 0.6616 & 0.6299 & 0.6835 & 0.6112 & 0.6564 & 0.7381 \\
\hline 208 & 0.8678 & 0.7206 & 0.6788 & 0.6466 & 0.7005 & 0.6277 & 0.6734 & 0.7568 \\
\hline 207 & 0.8859 & 0.7377 & 0.6958 & 0.6632 & 0.7177 & 0.6443 & 0.6906 & 0.7756 \\
\hline 206 & 0.9033 & 0.7545 & 0.7130 & 0.6798 & 0.7347 & 0.6608 & 0.7077 & 0.7942 \\
\hline 205 & 0.9209 & 0.7714 & 0.7297 & 0.6964 & 0.7515 & 0.6770 & 0.7248 & 0.8129 \\
\hline 204 & 0.9380 & 0.7881 & 0.7465 & 0.7128 & 0.7683 & 0.6933 & 0.7414 & 0.8319 \\
\hline 203 & 0.9561 & 0.8057 & 0.7638 & 0.7297 & 0.7859 & 0.7099 & 0.7589 & 0.8516 \\
\hline 202 & 0.9742 & 0.8239 & 0.7819 & 0.7475 & 0.8042 & 0.7272 & 0.7769 & 0.8718 \\
\hline 201 & 0.9946 & 0.8434 & 0.8017 & 0.7663 & 0.8243 & 0.7459 & 0.7970 & 0.8942 \\
\hline 200 & 1.0164 & 0.8646 & 0.8230 & 0.7876 & 0.8459 & 0.7667 & 0.8182 & 0.9184 \\
\hline 199 & 1.0412 & 0.8896 & 0.8478 & 0.8114 & 0.8706 & 0.7905 & 0.8430 & 0.9455 \\
\hline 198 & 1.0694 & 0.9159 & 0.8754 & 0.8384 & 0.8983 & 0.8172 & 0.8703 & 0.9765 \\
\hline 197 & 1.1038 & 0.9496 & 0.9091 & 0.8715 & 0.9324 & 0.8501 & 0.9039 & 1.0137 \\
\hline 196 & 1.1447 & 0.9896 & 0.9505 & 0.9116 & 0.9740 & 0.8902 & 0.9449 & 1.0583 \\
\hline 195 & 1.1912 & 1.0369 & 0.9966 & 0.9573 & 1.0203 & 0.9355 & 0.9909 & 1.1070 \\
\hline 194 & 1.2522 & 1.0971 & 1.0577 & 1.0171 & 1.0810 & 0.9952 & 1.0511 & 1.1701 \\
\hline 193 & 1.3263 & 1.1714 & 1.1320 & 1.0900 & 1.1552 & 1.0681 & 1.1246 & 1.2468 \\
\hline 192 & 1.4214 & 1.2675 & 1.2262 & 1.1833 & 1.2497 & 1.1616 & 1.2182 & 1.3425 \\
\hline 191 & 1.5545 & 1.3939 & 1.3595 & 1.3150 & 1.3819 & 1.2932 & 1.3503 & 1.4767 \\
\hline 190 & 1.6819 & 1.5252 & 1.4876 & 1.4420 & 1.5096 & 1.4208 & 1.4767 & 1.6043 \\
\hline
\end{tabular}




\begin{tabular}{|c|c|c|c|c|c|c|c|c|}
\hline \multicolumn{9}{|c|}{ In-line spectrophotometer (4mm path length) } \\
\hline $\begin{array}{c}\text { Wavelength } \\
(\mathrm{nm})\end{array}$ & $3.0 \mathrm{mg} / \mathrm{L}$ & $4.0 \mathrm{mg} / \mathrm{L}$ & $5.0 \mathrm{mg} / \mathrm{L}$ & $6.0 \mathrm{mg} / \mathrm{L}$ & $7.0 \mathrm{mg} / \mathrm{L}$ & $8.0 \mathrm{mg} / \mathrm{L}$ & $10.0 \mathrm{mg} / \mathrm{L}$ & $12.0 \mathrm{mg} / \mathrm{L}$ \\
\hline 189.8 & 0.7785 & 0.6798 & 0.6405 & 0.6365 & 0.6416 & 0.6306 & 0.6622 & 0.7692 \\
\hline 192.8 & 0.6805 & 0.5814 & 0.5426 & 0.5395 & 0.5451 & 0.5328 & 0.5631 & 0.6575 \\
\hline 195.8 & 0.6373 & 0.5388 & 0.5005 & 0.4970 & 0.5015 & 0.4907 & 0.5194 & 0.6082 \\
\hline 198.9 & 0.5956 & 0.4978 & 0.4602 & 0.4575 & 0.4614 & 0.4533 & 0.4800 & 0.5628 \\
\hline 201.9 & 0.5620 & 0.4665 & 0.4282 & 0.4256 & 0.4290 & 0.4193 & 0.4470 & 0.5258 \\
\hline 204.9 & 0.5215 & 0.4260 & 0.3897 & 0.3889 & 0.3916 & 0.3820 & 0.4082 & 0.4841 \\
\hline 208 & 0.4830 & 0.3879 & 0.3530 & 0.3500 & 0.3531 & 0.3442 & 0.3703 & 0.4426 \\
\hline 211 & 0.4491 & 0.3549 & 0.3193 & 0.3170 & 0.3193 & 0.3115 & 0.3372 & 0.4064 \\
\hline 214 & 0.4179 & 0.3232 & 0.2892 & 0.2870 & 0.2897 & 0.2821 & 0.3083 & 0.3750 \\
\hline 217 & 0.3881 & 0.2959 & 0.2597 & 0.2589 & 0.2618 & 0.2543 & 0.2791 & 0.3459 \\
\hline 220 & 0.3706 & 0.2749 & 0.2417 & 0.2399 & 0.2432 & 0.2358 & 0.2600 & 0.3254 \\
\hline 223.1 & 0.3507 & 0.2569 & 0.2226 & 0.2216 & 0.2248 & 0.2169 & 0.2409 & 0.3057 \\
\hline 226.1 & 0.3362 & 0.2438 & 0.2090 & 0.2091 & 0.2107 & 0.2037 & 0.2276 & 0.2910 \\
\hline 229.1 & 0.3240 & 0.2316 & 0.1982 & 0.1974 & 0.1992 & 0.1927 & 0.2159 & 0.2784 \\
\hline 232.1 & 0.3154 & 0.2223 & 0.1884 & 0.1876 & 0.1895 & 0.1847 & 0.2063 & 0.2678 \\
\hline 235.1 & 0.3079 & 0.2159 & 0.1824 & 0.1807 & 0.1828 & 0.1760 & 0.1991 & 0.2597 \\
\hline 238.1 & 0.3025 & 0.2089 & 0.1752 & 0.1740 & 0.1769 & 0.1698 & 0.1929 & 0.2527 \\
\hline 241.1 & 0.2969 & 0.2034 & 0.1702 & 0.1690 & 0.1721 & 0.1646 & 0.1876 & 0.2474 \\
\hline 244.1 & 0.2952 & 0.2008 & 0.1677 & 0.1663 & 0.1681 & 0.1615 & 0.1842 & 0.2445 \\
\hline 247.1 & 0.2912 & 0.1968 & 0.1625 & 0.1624 & 0.1638 & 0.1571 & 0.1796 & 0.2396 \\
\hline 250.1 & 0.2872 & 0.1928 & 0.1593 & 0.1583 & 0.1604 & 0.1538 & 0.1773 & 0.2348 \\
\hline
\end{tabular}




\begin{tabular}{|c|c|c|c|c|c|c|c|c|c|c|}
\hline \multicolumn{11}{|c|}{ Desktop spectrophotometer (1 $\mathrm{cm}$ path length) } \\
\hline $\begin{array}{c}\text { Wavelength } \\
(\mathrm{nm})\end{array}$ & $\begin{array}{l}0.025 \\
\mathrm{mg} / \mathrm{L}\end{array}$ & $\begin{array}{c}0.05 \\
\mathrm{mg} / \mathrm{L}\end{array}$ & $\begin{array}{c}0.1 \\
\mathrm{mg} / \mathrm{L}\end{array}$ & $\begin{array}{c}0.5 \\
\mathrm{mg} / \mathrm{L}\end{array}$ & $\begin{array}{c}1.0 \\
\mathrm{mg} / \mathrm{L}\end{array}$ & $\begin{array}{c}2.0 \\
\mathrm{mg} / \mathrm{L}\end{array}$ & $\begin{array}{c}4.0 \\
\mathrm{mg} / \mathrm{L}\end{array}$ & $\begin{array}{c}6.0 \\
\mathrm{mg} / \mathrm{L}\end{array}$ & $\begin{array}{c}8.0 \\
\mathrm{mg} / \mathrm{L}\end{array}$ & $\begin{array}{c}10.0 \\
\mathrm{mg} / \mathrm{L}\end{array}$ \\
\hline 250 & 0.7545 & 0.6536 & 0.6657 & 0.5377 & 0.3545 & 0.2676 & 0.2585 & 0.2692 & 0.2650 & 0.3145 \\
\hline 249 & 0.7595 & 0.6585 & 0.6705 & 0.5425 & 0.3594 & 0.2723 & 0.2632 & 0.2737 & 0.2698 & 0.3193 \\
\hline 248 & 0.7645 & 0.6634 & 0.6756 & 0.5475 & 0.3642 & 0.2772 & 0.2681 & 0.2785 & 0.2746 & 0.3241 \\
\hline 247 & 0.7694 & 0.6681 & 0.6803 & 0.5522 & 0.3689 & 0.2818 & 0.2727 & 0.2830 & 0.2793 & 0.3289 \\
\hline 246 & 0.7741 & 0.6728 & 0.6849 & 0.5566 & 0.3736 & 0.2862 & 0.2772 & 0.2876 & 0.2839 & 0.3334 \\
\hline 245 & 0.7785 & 0.6772 & 0.6893 & 0.5611 & 0.3778 & 0.2904 & 0.2815 & 0.2917 & 0.2881 & 0.3378 \\
\hline 244 & 0.7826 & 0.6813 & 0.6933 & 0.5652 & 0.3817 & 0.2943 & 0.2852 & 0.2958 & 0.2919 & 0.3418 \\
\hline 243 & 0.7862 & 0.6850 & 0.6971 & 0.5688 & 0.3852 & 0.2976 & 0.2886 & 0.2992 & 0.2955 & 0.3454 \\
\hline 242 & 0.7896 & 0.6883 & 0.7003 & 0.5718 & 0.3883 & 0.3007 & 0.2918 & 0.3024 & 0.2985 & 0.3487 \\
\hline 241 & 0.7925 & 0.6941 & 0.7045 & 0.5748 & 0.3910 & 0.3033 & 0.2944 & 0.3049 & 0.3013 & 0.3514 \\
\hline 240 & 0.7953 & 0.7025 & 0.7118 & 0.5767 & 0.3949 & 0.3056 & 0.2966 & 0.3071 & 0.3036 & 0.3538 \\
\hline 239 & 0.7974 & 0.7048 & 0.7169 & 0.5752 & 0.4059 & 0.3066 & 0.3006 & 0.3083 & 0.3048 & 0.3560 \\
\hline 238 & 0.7990 & 0.7065 & 0.7189 & 0.5770 & 0.4073 & 0.3179 & 0.3067 & 0.3196 & 0.3160 & 0.3568 \\
\hline 237 & 0.7981 & 0.7081 & 0.7203 & 0.5785 & 0.4086 & 0.3189 & 0.3034 & 0.3208 & 0.3173 & 0.3692 \\
\hline 236 & 0.7998 & 0.7094 & 0.7218 & 0.5797 & 0.4097 & 0.3201 & 0.2976 & 0.3218 & 0.3185 & 0.3705 \\
\hline 235 & 0.8013 & 0.7107 & 0.7230 & 0.5811 & 0.4107 & 0.3209 & 0.2985 & 0.3227 & 0.3195 & 0.3716 \\
\hline 234 & 0.8027 & 0.7120 & 0.7242 & 0.5822 & 0.4116 & 0.3218 & 0.2995 & 0.3235 & 0.3204 & 0.3729 \\
\hline 233 & 0.8044 & 0.7134 & 0.7256 & 0.5837 & 0.4126 & 0.3228 & 0.3005 & 0.3246 & 0.3216 & 0.3741 \\
\hline 232 & 0.8062 & 0.7148 & 0.7272 & 0.5849 & 0.4139 & 0.3239 & 0.3017 & 0.3256 & 0.3229 & 0.3755 \\
\hline 231 & 0.8084 & 0.7165 & 0.7289 & 0.5867 & 0.4151 & 0.3228 & 0.3030 & 0.3272 & 0.3222 & 0.3773 \\
\hline 230 & 0.8106 & 0.7188 & 0.7309 & 0.5886 & 0.4169 & 0.3245 & 0.3046 & 0.3287 & 0.3240 & 0.3796 \\
\hline 229 & 0.8135 & 0.7213 & 0.7333 & 0.5911 & 0.4190 & 0.3288 & 0.3067 & 0.3308 & 0.3283 & 0.3818 \\
\hline 228 & 0.8164 & 0.7240 & 0.7363 & 0.5938 & 0.4216 & 0.3313 & 0.3091 & 0.3332 & 0.3308 & 0.3846 \\
\hline 227 & 0.8202 & 0.7276 & 0.7396 & 0.5971 & 0.4247 & 0.3340 & 0.3120 & 0.3362 & 0.3340 & 0.3880 \\
\hline 226 & 0.8242 & 0.7316 & 0.7436 & 0.6008 & 0.4283 & 0.3374 & 0.3222 & 0.3398 & 0.3377 & 0.3920 \\
\hline 225 & 0.8294 & 0.7362 & 0.7483 & 0.6052 & 0.4325 & 0.3417 & 0.3330 & 0.3439 & 0.3420 & 0.3967 \\
\hline 224 & 0.8347 & 0.7415 & 0.7535 & 0.6102 & 0.4372 & 0.3465 & 0.3379 & 0.3490 & 0.3471 & 0.4020 \\
\hline 223 & 0.8409 & 0.7474 & 0.7595 & 0.6200 & 0.4428 & 0.3521 & 0.3435 & 0.3546 & 0.3529 & 0.4080 \\
\hline 222 & 0.8479 & 0.7541 & 0.7663 & 0.6348 & 0.4491 & 0.3584 & 0.3501 & 0.3611 & 0.3597 & 0.4149 \\
\hline 221 & 0.8557 & 0.7617 & 0.7739 & 0.6425 & 0.4563 & 0.3656 & 0.3573 & 0.3684 & 0.3673 & 0.4227 \\
\hline 220 & 0.8642 & 0.7701 & 0.7822 & 0.6511 & 0.4643 & 0.3738 & 0.3656 & 0.3766 & 0.3757 & 0.4316 \\
\hline 219 & 0.8736 & 0.7792 & 0.7914 & 0.6604 & 0.4732 & 0.3826 & 0.3747 & 0.3859 & 0.3854 & 0.4411 \\
\hline 218 & 0.8910 & 0.7893 & 0.8014 & 0.6705 & 0.4829 & 0.3926 & 0.3847 & 0.3961 & 0.3963 & 0.4521 \\
\hline 217 & 0.9057 & 0.7997 & 0.8119 & 0.6812 & 0.4929 & 0.4029 & 0.3953 & 0.4070 & 0.4080 & 0.4639 \\
\hline 216 & 0.9178 & 0.8106 & 0.8231 & 0.6927 & 0.5038 & 0.4143 & 0.4067 & 0.4188 & 0.4208 & 0.4767 \\
\hline
\end{tabular}




\begin{tabular}{|c|c|c|c|c|c|c|c|c|c|c|}
\hline 215 & 0.9303 & 0.8221 & 0.8347 & 0.7045 & 0.5152 & 0.4259 & 0.4187 & 0.4312 & 0.4343 & 0.4903 \\
\hline 214 & 0.9435 & 0.8340 & 0.8467 & 0.7170 & 0.5269 & 0.4381 & 0.4310 & 0.4441 & 0.4486 & 0.5048 \\
\hline 213 & 0.9569 & 0.8463 & 0.8666 & 0.7299 & 0.5394 & 0.4509 & 0.4443 & 0.4578 & 0.4638 & 0.5200 \\
\hline 212 & 0.9707 & 0.8710 & 0.8840 & 0.7432 & 0.5622 & 0.4638 & 0.4575 & 0.4715 & 0.4789 & 0.5394 \\
\hline 211 & 0.9849 & 0.8845 & 0.8976 & 0.7566 & 0.5777 & 0.4767 & 0.4709 & 0.4853 & 0.4941 & 0.5637 \\
\hline 210 & 0.9989 & 0.8984 & 0.9117 & 0.7699 & 0.5913 & 0.4899 & 0.4844 & 0.4991 & 0.5092 & 0.5800 \\
\hline 209 & 1.0131 & 0.9122 & 0.9256 & 0.7834 & 0.6046 & 0.5029 & 0.4974 & 0.5127 & 0.5324 & 0.5964 \\
\hline 208 & 1.0275 & 0.9262 & 0.9392 & 0.7967 & 0.6181 & 0.5243 & 0.5189 & 0.5393 & 0.5524 & 0.6125 \\
\hline 207 & 1.0414 & 0.9399 & 0.9528 & 0.8213 & 0.6312 & 0.5422 & 0.5369 & 0.5534 & 0.5675 & 0.6288 \\
\hline 206 & 1.0551 & 0.9534 & 0.9660 & 0.8349 & 0.6445 & 0.5559 & 0.5505 & 0.5674 & 0.5825 & 0.6447 \\
\hline 205 & 1.0686 & 0.9667 & 0.9795 & 0.8485 & 0.6575 & 0.5693 & 0.5642 & 0.5809 & 0.5973 & 0.6606 \\
\hline 204 & 1.0941 & 0.9801 & 0.9927 & 0.8620 & 0.6704 & 0.5828 & 0.5774 & 0.5949 & 0.6120 & 0.6768 \\
\hline 203 & 1.1085 & 0.9933 & 1.0060 & 0.8758 & 0.6834 & 0.5962 & 0.5911 & 0.6087 & 0.6267 & 0.6934 \\
\hline 202 & 1.1233 & 1.0069 & 1.0193 & 0.8896 & 0.6968 & 0.6100 & 0.6049 & 0.6227 & 0.6420 & 0.7106 \\
\hline 201 & 1.1384 & 1.0207 & 1.0406 & 0.9040 & 0.7122 & 0.6242 & 0.6191 & 0.6375 & 0.6581 & 0.7386 \\
\hline 200 & 1.1544 & 1.0478 & 1.0601 & 0.9189 & 0.7362 & 0.6392 & 0.6342 & 0.6533 & 0.6755 & 0.7616 \\
\hline 199 & 1.1718 & 1.0645 & 1.0773 & 0.9350 & 0.7527 & 0.6552 & 0.6503 & 0.6703 & 0.7017 & 0.7852 \\
\hline 198 & 1.1911 & 1.0811 & 1.0913 & 0.9533 & 0.7670 & 0.6774 & 0.6724 & 0.6972 & 0.7241 & 0.8072 \\
\hline 197 & 1.2020 & 1.0946 & 1.1062 & 0.9708 & 0.7822 & 0.6914 & 0.6867 & 0.7137 & 0.7433 & 0.8312 \\
\hline 196 & 1.2308 & 1.1157 & 1.1277 & 0.9969 & 0.8043 & 0.7182 & 0.7137 & 0.7377 & 0.7703 & 0.8622 \\
\hline 195 & 1.2614 & 1.1402 & 1.1522 & 1.0234 & 0.8290 & 0.7439 & 0.7399 & 0.7651 & 0.8003 & 0.9040 \\
\hline 194 & 1.2945 & 1.1804 & 1.1951 & 1.0548 & 0.8690 & 0.7750 & 0.7710 & 0.8047 & 0.8434 & 0.9509 \\
\hline 193 & 1.3402 & 1.2202 & 1.2330 & 1.0984 & 0.9088 & 0.8230 & 0.8195 & 0.8482 & 0.8903 & 0.9983 \\
\hline 192 & 1.3995 & 1.2755 & 1.2887 & 1.1558 & 0.9636 & 0.8757 & 0.8720 & 0.9027 & 0.9509 & 1.0684 \\
\hline 191 & 1.4565 & 1.3336 & 1.3477 & 1.2143 & 1.0238 & 0.9365 & 0.9310 & 0.9673 & 1.0162 & 1.1421 \\
\hline 190 & 1.5356 & 1.4114 & 1.4313 & 1.2978 & 1.1007 & 1.0154 & 1.0127 & 1.0454 & 1.0973 & 1.2264 \\
\hline
\end{tabular}




\begin{tabular}{|c|c|c|c|c|c|c|c|c|c|c|}
\hline \multicolumn{11}{|c|}{ Hydrex 3661 optimum dose determination low turbidity Rideau River water (546 NTU) } \\
\hline \multicolumn{11}{|c|}{ In-line spectrophotometer (4mm path length) } \\
\hline $\begin{array}{c}\text { Wavelength } \\
\text { (nm) }\end{array}$ & $\begin{array}{l}0.025 \\
\mathrm{mg} / \mathrm{L}\end{array}$ & $\begin{array}{c}0.05 \\
\mathrm{mg} / \mathrm{L}\end{array}$ & $\begin{array}{c}0.1 \\
\mathrm{mg} / \mathrm{L}\end{array}$ & $\begin{array}{c}0.5 \\
\mathrm{mg} / \mathrm{L}\end{array}$ & $\begin{array}{c}1.0 \\
\mathrm{mg} / \mathrm{L}\end{array}$ & $\begin{array}{c}2.0 \\
\mathrm{mg} / \mathrm{L}\end{array}$ & $\begin{array}{c}4.0 \\
\mathrm{mg} / \mathrm{L}\end{array}$ & $\begin{array}{c}6.0 \\
\mathrm{mg} / \mathrm{L}\end{array}$ & $\begin{array}{c}8.0 \\
\mathrm{mg} / \mathrm{L}\end{array}$ & $\begin{array}{r}10.0 \\
\mathrm{mg} / \mathrm{L}\end{array}$ \\
\hline 189.8 & 0.9751 & 0.9119 & 0.8888 & 0.6290 & 0.4532 & 0.3987 & 0.4370 & 0.4871 & 0.5378 & 0.5993 \\
\hline 192.8 & 0.9010 & 0.8538 & 0.8243 & 0.5716 & 0.4017 & 0.3467 & 0.3827 & 0.4263 & 0.4679 & 0.5158 \\
\hline 195.8 & 0.8671 & 0.8238 & 0.7930 & 0.5419 & 0.3740 & 0.3207 & 0.3590 & 0.3949 & 0.4320 & 0.4722 \\
\hline 198.9 & 0.8329 & 0.7965 & 0.7600 & 0.5116 & 0.3413 & 0.2953 & 0.3277 & 0.3654 & 0.3982 & 0.4341 \\
\hline 201.9 & 0.8021 & 0.7707 & 0.7336 & 0.4886 & 0.3180 & 0.2737 & 0.3104 & 0.3404 & 0.3701 & 0.4035 \\
\hline 204.9 & 0.7726 & 0.7412 & 0.7042 & 0.4607 & 0.2941 & 0.2484 & 0.2777 & 0.3110 & 0.3362 & 0.3667 \\
\hline 208 & 0.7481 & 0.7139 & 0.6754 & 0.4349 & 0.2705 & 0.2258 & 0.2571 & 0.2832 & 0.3033 & 0.3320 \\
\hline 211 & 0.7231 & 0.6927 & 0.6519 & 0.4120 & 0.2461 & 0.2039 & 0.2310 & 0.2593 & 0.2770 & 0.3051 \\
\hline 214 & 0.7037 & 0.6727 & 0.6320 & 0.3925 & 0.2256 & 0.1862 & 0.2129 & 0.2402 & 0.2562 & 0.2837 \\
\hline 217 & 0.6843 & 0.6546 & 0.6118 & 0.3733 & 0.2097 & 0.1698 & 0.1963 & 0.2227 & 0.2377 & 0.2649 \\
\hline 220 & 0.6687 & 0.6397 & 0.5986 & 0.3590 & 0.1985 & 0.1580 & 0.1840 & 0.2102 & 0.2247 & 0.2512 \\
\hline 223.1 & 0.6557 & 0.6271 & 0.5826 & 0.3469 & 0.1836 & 0.1452 & 0.1717 & 0.1973 & 0.2113 & 0.2377 \\
\hline 226.1 & 0.6464 & 0.6207 & 0.5730 & 0.3376 & 0.1765 & 0.1377 & 0.1691 & 0.1892 & 0.2027 & 0.2286 \\
\hline 229.1 & 0.6390 & 0.6132 & 0.5657 & 0.3293 & 0.1716 & 0.1308 & 0.1602 & 0.1821 & 0.1952 & 0.2209 \\
\hline 232.1 & 0.6331 & 0.6076 & 0.5582 & 0.3245 & 0.1665 & 0.1263 & 0.1525 & 0.1764 & 0.1895 & 0.2150 \\
\hline 235.1 & 0.6287 & 0.6048 & 0.5554 & 0.3199 & 0.1651 & 0.1237 & 0.1495 & 0.1726 & 0.1858 & 0.2101 \\
\hline 238.1 & 0.6263 & 0.6011 & 0.5526 & 0.3163 & 0.1651 & 0.1197 & 0.1465 & 0.1688 & 0.1819 & 0.2065 \\
\hline 241.1 & 0.6246 & 0.5968 & 0.5486 & 0.3115 & 0.1597 & 0.1154 & 0.1421 & 0.1650 & 0.1782 & 0.2027 \\
\hline 244.1 & 0.6208 & 0.5977 & 0.5462 & 0.3092 & 0.1529 & 0.1128 & 0.1400 & 0.1612 & 0.1752 & 0.1990 \\
\hline 247.1 & 0.6198 & 0.5956 & 0.5426 & 0.3050 & 0.1525 & 0.1099 & 0.1352 & 0.1569 & 0.1707 & 0.1947 \\
\hline 250.1 & 0.6197 & 0.5952 & 0.5409 & 0.3021 & 0.1511 & 0.1059 & 0.1320 & 0.1544 & 0.1686 & 0.1919 \\
\hline
\end{tabular}




\section{Appendix E: Particle count data for experimental phase 4}

\begin{tabular}{|c|c|c|c|c|c|c|c|c|c|}
\hline \multicolumn{7}{|c|}{ Magnafloc High Turbidity Particle Count Data } \\
\hline \multirow{2}{*}{$\begin{array}{c}\text { Polymer Dose } \\
(\mathbf{m g} / \mathbf{L})\end{array}$} & 4 & 10 & 22 & 46 & 94 & 190 & 382 & 766 & $\begin{array}{c}\text { Total Particle } \\
\text { Concentration }\end{array}$ \\
\cline { 2 - 10 } & \multicolumn{7}{|c|}{ Median Floc Size } \\
\hline 0.1 & 397101.7 & 122586.1 & 12248.1 & 3407.4 & 3039.0 & 1749.7 & 92.1 & 0.0 & 540224.09 \\
\hline 0.5 & 353370.0 & 113790.0 & 25791.5 & 2657.0 & 292.0 & 36.0 & 1.0 & 0.0 & 495937.50 \\
\hline 1.0 & 276033.0 & 74516.0 & 14520.5 & 1327.5 & 65.0 & 6.5 & 0.0 & 0.0 & 366468.50 \\
\hline 1.5 & 185249.5 & 44618.5 & 10273.0 & 1455.5 & 104.0 & 4.5 & 0.0 & 0.0 & 241705.00 \\
\hline 2.0 & 181034.5 & 44594.0 & 8805.5 & 1159.0 & 195.5 & 8.0 & 0.0 & 0.0 & 235796.50 \\
\hline 3.0 & 217685.0 & 57697.5 & 13436.5 & 2485.5 & 369.5 & 17.0 & 0.0 & 0.0 & 291691.00 \\
\hline 4.0 & 247450.5 & 67325.0 & 15626.0 & 2384.5 & 257.0 & 24.0 & 0.0 & 0.0 & 333067.00 \\
\hline 5.0 & 283130.5 & 73342.0 & 16718.0 & 2919.5 & 252.5 & 20.5 & 0.0 & 0.0 & 376383.00 \\
\hline
\end{tabular}

\begin{tabular}{|c|c|c|c|c|c|c|c|c|c|}
\hline \multicolumn{7}{|c|}{ Magnafloc Low Turbidity Particle Count Data } \\
\hline $\begin{array}{c}\text { Polymer } \\
\text { Dose (mg/L) }\end{array}$ & \multicolumn{7}{|c|}{ Median Floc Size } & \multicolumn{2}{c|}{$\begin{array}{c}\text { Total Particle } \\
\text { Concentration }\end{array}$} \\
\cline { 2 - 11 } & 4 & 10 & 22 & 46 & 94 & 190 & 382 & 766 & 293920.50 \\
\hline 0.05 & 190442.50 & 80653.50 & 17280 & 3187 & 1117 & 661.5 & 382 & 197.00 & 211097.00 \\
\hline 0.1 & 129433.00 & 61007.5 & 17644 & 2708 & 295 & 9 & 0.5 & 0 & 242998.00 \\
\hline 0.5 & 136396.00 & 73345.5 & 26933 & 5798 & 520 & 5 & 0 & 0.50 & 263820.00 \\
\hline 1.0 & 152846.00 & 82272.5 & 25128.5 & 3357 & 208 & 8 & 0 & 0 & 286646.00 \\
\hline 1.5 & 166951 & 89974.5 & 26481.5 & 3168.5 & 69.5 & 1 & 0.00 & 0.00 & 307055.00 \\
\hline 2.0 & 172991.50 & 94733.5 & 31868.5 & 6737 & 712.5 & 11.5 & 0.5 & 0.00 & 321332.00 \\
\hline 3.0 & 181549.00 & 101563 & 33523.5 & 4448 & 242 & 6 & 0.5 & 0.00 & 3 \\
\hline
\end{tabular}

\begin{tabular}{|c|c|c|c|c|c|c|c|c|c|}
\hline \multicolumn{7}{|c|}{ Magnafloc High Turbidity with Actiflo Particle Count Data } \\
\hline \multirow{2}{*}{$\begin{array}{c}\text { Polymer } \\
\text { Dose (mg/L) }\end{array}$} & \multicolumn{7}{|c|}{ Median Floc Size } & $\begin{array}{c}\text { Total Particle } \\
\text { Concentration }\end{array}$ \\
\cline { 2 - 11 } & 4 & 10 & 22 & 46 & 94 & 190 & 382 & 766 & 302666.00 \\
\hline 0.05 & 139506.00 & 67449.00 & 44358.5 & 30395 & 16159 & 4481 & 314.5 & 3.00 & 143834.00 \\
\hline 0.1 & 93535.00 & 31409.5 & 13373.5 & 4224 & 676 & 463.5 & 123 & 29.5 & 106877.50 \\
\hline 0.5 & 78695.00 & 22169 & 5246 & 602 & 42.5 & 28.5 & 52 & 42.50 & 450853.00 \\
\hline 1.0 & 341928.00 & 88265.5 & 17377 & 2835.5 & 366 & 42.5 & 20 & 18.5 & 440455.00 \\
\hline 2.0 & 339985 & 75025 & 20729.5 & 4138 & 516 & 50 & 8.00 & 3.50 & 512307.50 \\
\hline 4.0 & 401289.00 & 85267.5 & 21185 & 4022 & 471 & 64 & 7.5 & 1.50 & 501572.50 \\
\hline 6.0 & 407761.50 & 73518.5 & 16492 & 3313 & 376 & 77 & 28 & 6.50 & 636443.50 \\
\hline 8.0 & 504940.50 & 106291.00 & 21671.00 & 3012.00 & 413.00 & 103.50 & 12.00 & 0.50 & 6 \\
\hline
\end{tabular}




\begin{tabular}{|c|c|c|c|c|c|c|c|c|c|}
\hline \multicolumn{10}{|c|}{ Hydrex 3661 High Turbidity Particle Count Data } \\
\hline \multirow{2}{*}{$\begin{array}{l}\text { Polymer Dose } \\
(\mathrm{mg} / \mathrm{L})\end{array}$} & \multicolumn{8}{|c|}{ Median Floc Size } & \multirow{2}{*}{$\begin{array}{l}\text { Total Particle } \\
\text { Concentration }\end{array}$} \\
\hline & 4 & 10 & 22 & 46 & 94 & 190 & 382 & 766 & \\
\hline 3 & 66846.00 & 16329.50 & 4928 & 1082 & 188 & 46 & 8.5 & 0.00 & 89428.00 \\
\hline 4 & 23698.00 & 7188.5 & 2653.5 & 612 & 80.5 & 9 & 1.5 & 0.5 & 34243.50 \\
\hline 5 & 24883.50 & 9883 & 4573.5 & 1345.5 & 179.5 & 13.5 & 2.5 & 0.00 & 40881.00 \\
\hline 6 & 18559.50 & 6455 & 2628 & 701.5 & 110.5 & 12.5 & 3.5 & 0.5 & 28471.00 \\
\hline 7 & 14186 & 5129 & 2027.5 & 445.5 & 53.5 & 2.5 & 0.00 & 0.00 & 21844.00 \\
\hline 8 & 13824.00 & 5145.5 & 2255 & 579.5 & 91.5 & 7.5 & 0.5 & 0.00 & 21903.50 \\
\hline 10 & 22279.00 & 8301 & 3147 & 690.5 & 67.5 & 2 & 0.5 & 0.00 & 34487.50 \\
\hline 12 & 76258.00 & 28974.50 & 10922.00 & 2399.00 & 172.50 & 6.50 & 1.00 & 0.00 & 118733.50 \\
\hline
\end{tabular}

\begin{tabular}{|c|c|c|c|c|c|c|c|c|c|}
\hline \multicolumn{7}{|c|}{ Hydrex 3661 Low Turbidity Particle Count Data } \\
\hline $\begin{array}{c}\text { Polymer } \\
\text { Dose (mg/L) }\end{array}$ & \multicolumn{7}{|c|}{ Median Floc Size } & \multicolumn{2}{c|}{$\begin{array}{c}\text { Total Particle } \\
\text { Concentration }\end{array}$} \\
\cline { 2 - 10 } & 4 & 10 & 22 & 46 & 94 & 190 & 382 & 766 & 789048.00 \\
\hline 0.025 & 386280.5 & 240004.0 & 126156.0 & 35303.0 & 1300.5 & 2.0 & 2.0 & 0.0 & 760726.00 \\
\hline 0.050 & 363299.5 & 226738.5 & 123514.5 & 43011.0 & 4147.5 & 15.0 & 0.0 & 0.0 & 577583.50 \\
\hline 0.10 & 257034.0 & 171155.0 & 105582.5 & 40089.0 & 3709.5 & 13.5 & 0.0 & 0.0 & 54073.50 \\
\hline 0.50 & 42006.5 & 8562.5 & 2649.0 & 666.0 & 122.5 & 31.0 & 17.5 & 18.5 & 16684.50 \\
\hline 1.0 & 12669.0 & 2963.0 & 786.0 & 199.0 & 37.0 & 3.0 & 6.5 & 21.0 & 21256.50 \\
\hline 2.0 & 17807.5 & 3143.0 & 247.0 & 35.5 & 19.0 & 4.0 & 0.5 & 0.0 & 35494.00 \\
\hline 4.0 & 26860.5 & 6865.0 & 1334.0 & 270.0 & 141.0 & 23.5 & 0.0 & 0.0 & 80127.00 \\
\hline 6.0 & 55160.5 & 17473.5 & 5427.0 & 1722.5 & 337.0 & 6.0 & 0.5 & 0.0 & 130739.00 \\
\hline 8.0 & 92292.5 & 29168.0 & 7111.0 & 1687.5 & 393.5 & 86.5 & 0.0 & 0.0 & 154511.50 \\
\hline 10.0 & 105614.5 & 35863.5 & 10245.0 & 2304.5 & 451.0 & 32.5 & 0.5 & 0.0 & \\
\hline
\end{tabular}




\section{REFERENCES}

1. Coagulants and natural polymers: perspectives for the treatment of water. Theodoro, J., et al. 3, 2013, Plast Polym Technol (PAPT), Vol. 2, pp. 55-62.

2. field trials of appropriate hydraulic flocculation processes. McConnachie, G., Folkard, G. and Mtawali, M. 6, 1999, Water Resources, Vol. 33, pp. 1425-1434.

3. Kim, Young. Coagulants and Flocculants: Theory and Practice. Littleton, CO : Tall Oaks Ventures LLC, 2015.

4. Bolto, B., et al. The Use of Cationic Polymers as Primary Coagulants in Water Treatment. Chemical Water and Wastewater Treatment V. Berlin: Springer, Berlin, Heidelberg, 1998, pp. 173-185.

5. Organic Polyelectrolytes in Water Treatment. Bolto, Brian and Gregory, John. 2007, Water Research Joural, pp. 2301-2324.

6. How Safet are Organic Polymers in Water Treatment? Mallevaille, Joel, Bruchet, Auguste and Fiessinger, Francois. 1984, American Water Works Association , pp. 8793.

7. Enforcement, EPA Office of Environmental. Advice Note No. 15: Optimisation of Chemical Coagulant Dosing at Water Treatment Works. Wexford, Ireland : Environmental Protection Agency (EPA), 2014.

8. Clogging of filter medium by excess polymer during alum sludge filtration. Zhao, Y., Papavasilopoulos, E. and Bache, D. 1998, Filtration and Separation, pp. 947-950, 946.

9. Polyelectrolyte determination in drinking water. Majam, S and Thompson, PA. 2006, Water SA, pp. 705-707.

10. Organization, World Health. Guidelines for Drinking Water Quality, 4th ed. Geneva : World Health Organization, 2017.

11. Nnaji, P. An investigation of the performance of various coagulants/flocculants in removing the turbidity of coal washery effluents. Owerri: Federal University of Technology, 2012.

12. (AWWA), American Water Works Association. Operational Control of Coagulation and Filtration Processes. Manual of Water Supply Practices M37. Denver, CO : AWWA, 2011. 
13. Mels, A., van Nieuwenhuijzen, A. and Klapwijk, A. Turbidity related dosing of organic polymers to control the denitrification potential of flocculated municipal wastewater. Chemical Water and Wastewater Treatment VII. London : IWA Publishing, 2002, pp. 71-80.

14. feed-forward automation for cost effectivechemical treatment of food manufacturing wastewater. Norman, P. and Espinoza, C. New Orleans : s.n., 2014. Water Technologies \& Solutions Technical Paper.

15. State of the Art of Online Monitoring and Control of the Coagulation Process . Ratnaweera, H. and Fettig, J. 2015, Water, Vol. 7, pp. 6574-6597.

16. Chemical feed control using coagulation computer models and a streaming current detector. Yavich, A.A. and van de Wege, J. 2013, Water Sci. Technology, Vol. 67, pp. 2814-2821.

17. Influence of streaming potential on flux decline of microfiltration with in-line rapid pre-coagulation process for drinking water production. Oh, J.I. and Lee, S.H. 2005, J. Membr. Sci., Vol. 254, pp. 39-47.

18. Streaming current titration for coagulation of high turbidity water. Nam, S.W., et al. 2013, Colloids Surf. A Physicochem. Eng. Asp., Vol. 419, pp. 133-139.

19. Aluminium sulfate as coagulant for highly polluted cork processing wastewaters: Removal of organic matter. Domiguez, Joaquin, et al. 2007, Journal of Hazardous Materials, pp. 15-21.

20. Alternative cost-effective preparation method of PAC coagulant agent: Characterization and comparative application for water/wastewater treatment. Zouloubis, A.I. and Tzoupanos, N. 2010, Desalination, Vol. 250, pp. 339-344.

21. Automatic Control for Chemical Dosing in Laboratory-scale Coagulation Process by Using an Optical Monitor. Huang, C. and Liu, C.B. 1996, Water Resources, Vol. 30, pp. 1924-1929.

22. Measurement of polyacrylamide polymers in water and wastewater using an in-line $U V$-vis spectrophotometer. Al Momani, Fares and Ormeci, Banu. 2014, Journal of Environmental Chemical Engineering, pp. 765-772.

23. Optimization of Polymer Dose Based on Residual Polymer Concentration in Dewatering Supernatant. Al Momani, Fares and Ormeci, Banu. Swittzerland : Springer International, 2014, Vol. 2154.

24. The effect of path length, light intensity and co-added time on the detection limit associated with NIR spectroscopy of potassium hydrogen phthalate in aqueous solution. 
Inagaki, T., Watanabe, T. and Tsuchikawa, S. 1, s.1. : PLOS One, 2017, PLOS One, Vol. 12, p. 5.

25. Frankel, M. Water Treatment and Purification. Facility Piping Systems Handbook. New York, NY : The McGraw-Hill Companies, Inc., 2002, p. Chapter 4.

26. Cheremisinoff, N. Handbook of Water and Wastewater Treatment Technologies. Woodburn, MA : Butterworth-Heinemann, 2002.

27. Parsons, S. and Jefferson, B. Introduction to Potable Water Treatment Processes. Oxford, UK : Blackwell Publishing Ltd., 2006.

28. Natural organic matter removal by coagulation during drinking water treatment: A review. Matilainen, Anu, Vepsäläinen, Mikko and Sillanpää, Mika. 2010, Advances in Colloid and Interface Science, pp. 189-197.

29. Ogedengbe, M. Polyelectrolytes in the treatment of wastewaters. Ames, IA : Iowa State University Retrospective Theses and Dissertations, 1972.

30. Role of surface silanol groups in flocculation of silica suspensions by polyacrylamide. Griot, O. and Kitchener, J.A. 1965, Chemistry of adsorption process, pp. 1026-1031.

31. Flocculation and de-watering of kimberlite clay slimes. O'Gorman, J.V. and Kitchener, J.A. 1974, Int. J. Miner. Process, pp. 33-49.

32. Filtration of colloidal dispersions flocculated by anionic and cationic polyelectrolytes. La Mer, V.K. 1966, Disc. Faraday Soc., pp. 248-254.

33. Addressing water scarcity: cationic polyelectrolytes in water treatment and purification. Wilts, Emily, Herzberger, Jana and Long, Timothy. 2018, Polymer International , pp. 799-814.

34. Criddle, J. A review of the mammalian and aquatic toxicity of polyelectrolytes. NR 2545, Medmenham : Foundation for Water Research, 1990.

35. De Rosemond, S. and Liber, K. Wastewater Treatment Polymers Identified as the Toxic Component of a Diamond Mine Effluent. Saskatoon, SK : Environmental Toxicology and Chemistry, 2004.

36. Long term experience in the use of polymeric coagulants at Umgeni Water. Noziac, D., Freese, S. and Thompson, P. 2001, Water Sci. Technol.: Water Supply, pp. 43-50.

37. Acrylamide copolymers: A review of methods for determination of concentration and degree of hydrolysis. Taylor, K.C. and Nasr-El-Din, H.A. 1994, Petroleum Science and Engineering, pp. 9-23. 
38. Controlling Coagulation Process: From Zeta Potential to Streaming Potential. Ghernaout, D., et al. 2015, American Journal of Environmental Protection. Special Issue: Cleaner and Sustainable Production. , pp. 16-27.

39. Quantification of polymer concentration in water using UV-Vis spectroscopy. Gibbons, Meaghan and Ormeci, Banu. 2013, Journal of Water Supply: Research and Technology, pp. 205-213.

40. Perkampus, H.-H. UV-Vis Spectroscopy and its Applications. Berlin : SpringerVerlag, 1992.

41. Forster, H. V/VIS Spectroscopy. [book auth.] H.G. Karge and J Weitkamp. Characterization I. Molecular Sieves - Science and Technology. Berlin, Heidelberg: Springer, 2004, pp. 338-421.

42. Industrial polymer effluent treatment by chemical coagulation and flocculation. Sher, Farooq, Malik, Atig and Liu, Hao. 2013, Journal of Environmental Chemical Engineering, pp. 684-689.

43. Optimizing the coagulation process in a drinking water treatment plant-comparison between traditional and statistical experimental design jar tests. Zainal-Abideen, M., et al. 2012, Water Science and Technology, pp. 496-503.

44. Satterfield, Z. Tech Brief: Jar Testing. Morgantown, WV: The National Environmental Services Center, 2005.

45. Torfs, E., et al. Settling Tests. Experiemental Methods in Wastewater Treatment. London, UK : IWA Publishing, 2016, pp. 235-262.

46. Screening and evaluation of polymers as flocculation aids for the treatment of aquacultural effluents. Ebeling, J., Rishel, K. and Sibrell, P. 4, 2005, Aquacultural Engineering, Vol. 33, pp. 235-249.

47. Determination of the optimum polymer dose for dewatering of oil sands tailings using UV-vis spectrophotometry. Salam, A., Ormeci, B. and Simms, P. 2016, Journal of Petroleum Science and Engineering, Vol. 147, pp. 68-76.

48. Meunier, John. Actiflo and Dunsenflo Package Plants Fact Sheet. Saint-Laurent, QC : Veolia Water Solutions \& Technologies Inc., 2008.

49. Spectroscopic characterization of water extractable organic matter during composting of municipal solid waste. He, Xiaosong, et al. 2011, Chemosphere, pp. 541-548.

50. Determination of organic matter by UV absorption in the ground water. Albrektienè, Ramuné, et al. 2012, Journal of Environmetal Engineering and Landscape Management, pp. 163-167. 
51. Liquid Chromotographic Determination of Acrylamide Monomer in Natural and Polluted Aqueous Environments. Brown, L, and Rhead, M. 1979, Analyst, Vol. 104, pp. 391-399.

52. Determination of Acrylamide Monomer in Polyacrylamide Degradation Studies by High-Performance Liquid Chromatography. Ver Vers, L. 1999, Journal of Chromatographic Science, Vol. 37, pp. 486-494.

53. The determination of acrylamide in environmental and drinking waters by largevolume injection - hydrophilic-interaction liquid chromatography and tandem mass spectrometry. Backe, W., Yingling, V. and Johnson, T. 2014, Journal of Chromatography A, Vol. 1334, pp. 72-78.

54. Laboratory studies on the adsorption of acrylamide monomer by sludge, sediments, clays, peat and synthetic resins. Brown, L., Bancroft, K. and Rhead, M. 7, 1980, Water Research, Vol. 14, pp. 779-781.

55. The Determination of Acrylamide in Water by Using Electron-capture Gas Chromatography. Croll, B.T. and Simkins, G.M. 1972, Analyst, Vol. 97, pp. 281-288.

56. Rapid determination of ultra-trace amounts of acrylamide contaminant in water samples using dispersive liquid-liquid microextraction coupled to gas chromatographyelectron capture detector. Yamini, Y., et al. 13, 2012, International Journal of Environmental Analytical Chemistry, Vol. 92, pp. 1493-1505.

57. Brown, L. C. and Bertouex, P.M. Statistics for Environmental Engineers, 2nd ed. Boca Raton, FL. : CRC Press, 2002. 